Portland State University

PDXScholar

Summer 7-19-2013

\title{
Developing Thyronamine Analog Pharmaceuticals Targeting TAAR1 to Treat Methamphetamine Addiction
}

Troy Andrew Wahl

Portland State University

Follow this and additional works at: https://pdxscholar.library.pdx.edu/open_access_etds

Part of the Chemicals and Drugs Commons, and the Medicinal-Pharmaceutical Chemistry Commons Let us know how access to this document benefits you.

Recommended Citation

Wahl, Troy Andrew, "Developing Thyronamine Analog Pharmaceuticals Targeting TAAR1 to Treat Methamphetamine Addiction" (2013). Dissertations and Theses. Paper 1109.

https://doi.org/10.15760/etd.1109

This Dissertation is brought to you for free and open access. It has been accepted for inclusion in Dissertations and Theses by an authorized administrator of PDXScholar. Please contact us if we can make this document more accessible: pdxscholar@pdx.edu. 
Developing Thyronamine Analog Pharmaceuticals Targeting TAAR1 to Treat

Methamphetamine Addiction

by

Troy Andrew Wahl

A dissertation submitted in partial fulfillment of the requirements for the degree of

Doctor of Philosophy

in

Chemistry

Dissertation Committee:

David H. Peyton, Chair

Suzanne Estes

David Grandy

Dirk Iwata-Reuyl

Robert Strongin

Portland State University

2013 
(C) 2013 Troy Andrew Wahl 


\section{Abstract}

As a part of the overall program in the Grandy laboratory at Oregon Health \& Science University (OHSU), studying the underlying chemical biology of methamphetamine (Meth) addiction, this dissertation reports on the development of six new thyronamine analogs which were synthesized and assayed against trace amine associated receptor 1 (TAAR1), giving preliminary results consistent with the analogs being inverse agonists. Due to highly variable TAAR1 expression levels in the assays, based on inter-assay response to control Meth stimulation as well as other possible factors, kinetic models were developed to qualitatively explain the assay results. The models set approximate limits on the analogs' binding and disassociation rates relative to those of Meth. Analysis of the assays also provides more evidence of TAAR1's basal activity. Based on the models, the conversion rate of ligand-free inactive TAAR1 to ligand-free active TAAR1 is less than $6 \%$ of the binding rate of Meth to TAAR1. The models also suggest that the inverse agonists bind to the inactive ligand-free form of TAAR 1 between 10 and 100 times faster than Meth binds to the inactive ligand-free form of TAAR1. Three of the new ana$\operatorname{logs}$, G5-110s8, G5-112s5, and G5-114s5, bind to the ligand-free active form of TAAR1 faster than they bind to the inactive ligand-free form of TAAR1. The models do not suggest an upper limit on the binding rate of those 3 analogs to the ligand-free active form of TAAR1. A control assay lacking TAAR1 revealed an electrophysiological off-target effect caused by G5-109s8. Also, a novel synthetic 
route was developed for ET-92, the lead compound for this project, which reduced the number of synthetic steps from 14 to 5 and improved the overall yield from $15.3 \%$ to $18.3 \%(77.4 \mathrm{mg})$ with the hope that further improvements in yield are possible. 


\section{Acknowledgments}

Working with Dr. Grandy, a renowned expert on TAAR1 and who laid the foundation for this work by proposing the connection between TAAR1 and Meth addiction, was greatly appreciated. It is also appreciated that Oregon Health \& Sciences University hosted this research.

The following people provided additional support in the form of materials, and advice:

- Dr. Niles Lehman

- Dr. Dirk Iwata-Reuyl

- Dr. David Peyton

- Dr. Rolf Winter
- Dr. Michael Cohen

- Dr. David Dawson

- Dr. Thomas Scanlan

- Dr. Yohei Norimatsu

With special thanks to my volunteer "editorial staff:"

- Cheryl Hodson

- Rob Jensen

- Katherine Liebman

- Kelsie Kendrick
- Natasja Swartz

- Kimberly Wahl

- Sheila Wahl

Emma Florence Martin Holt and Sheila Wahl provided personal financial aid which is greatly appreciated.

This research was made possible, in part, by funding from the National Institute on Drug Abuse, The Portland Oregon Methamphetamine Research Center, The Medical Research Foundation of Oregon, The Steinberg Fund and the Department of Physiology \& Pharmacology, School of Medicine, Oregon Health \& Science University. 
The following people have my gratitude for their help and mentoring over the years independent of their involvement in the project:

\section{PhD Committee Members}

Dr. Suzanne Estes

Dr. David Grandy

Dr. Dirk Iwata-Reuyl

Dr. David H. Peyton

Dr. Robert Strongin

\section{Grandy Research Group at OHSU}

Dr. David Grandy Anthony Beers

Madeline Grandy

Ashley Kimbel

Katie Tallman

\section{Others at OHSU}

Dr. Michael Cohen

Dr. David Dawson

Dr. Jeffrey Karpen

Dr. Yohei Norimatsu

Dr. Thomas Scanlan

\section{Peyton Research Group at PSU}

Dr. David Peyton

Cheryl Hodson

Rob Jensen

Katherine Liebmann

Kelsie Kendrick

\section{Simoyi Research Group at PSU}

Dr. Rueben Simoyi

Wilbes Mbiya

\section{Goforth Research Group at PSU}

Dr. Andrea Goforth

William DeBenedetti

Beth Manhat

\section{Others at PSU}

Kirk Fisher

Keith James

Dr. Niles Lehman

Dr. David McClure

Nicolas Meier

Dr. Keith Schwartz

Dr. Eric Sheagley

Dr. Carl Wamser

Dr. Rolf Winter

\section{Celera Genomics}

Dr. Dan Dickman

Dr. Paul Grothaus

Dr. Orion Jankowski

Dr. John Link

Dr. Ellen Leah

Colin O'Bryan

Dr. Jennifer Riggs

Dr. William Shrader

Dr. Kieron Wesson

Dr. Wendy Young

Sheila Zipfel, MS

\section{Cellgate}

Dr. Tad Jessop

Dr. Thorsten Kirschberg

Dr. Paul McGrane

Dr. Sriram Naganathan

Dr. Jonathan Rothbard

\section{Peninsula Laboratories/Bachem}

Dr. Alfred Chen

Dr. Ruoheng Zhang

\section{Others}

Raymond King - AboutUs

Brian Lundquist - Nanotechnology Now 


\section{Table of Contents}

Abstract

Acknowledgments $\quad$ iii

List of Tables $\quad \mathrm{x}$

List of Figures $\quad$ xi

CHAPTER 1 A New Approach for Treating Methamphetamine Addiction 1

$1.1 \quad$ Background on Trace Amine Associated Receptor 1 (TAAR1) 1

$1.2 \quad$ Methamphetamine 5

$1.3 \quad$ Justification for targeting TAAR1 7

1.4 Overview of Current TAAR1 Medicinal Chemistry 11

CHAPTER 2 New ET-92 Synthesis 14

2.1 Cost-effective synthetic route to

ET-92 and its analogs 14

CHAPTER 3 New ET-92 Analogs 23

3.1 Initial attempts to synthesize new analogs 23

3.2 Change in strategy and targets 24

3.3 Another strategy change 27

3.4 Final attempt to synthesize $6-14$

CHAPTER 4 Biological Results 32

4.1 The experiments 33

$\begin{array}{lll}4.2 & \text { Results } & 37\end{array}$

$\begin{array}{lll}\text { 4.2.1 Testing G5-109s8 } & 37\end{array}$

4.2.2 Testing the other compounds 45

4.3 Concluding remarks about the assays 49 
Table of Contents

CHAPTER 5 Kinetic Modeling

5.1 Quick Review of Kinetics 51

5.2 TAAR1 Kinetics 52

5.3 Model B: Modeling TAAR1 equilibria 55

5.4 Model C: Modeling the oocyte data 60

5.5 Stop Flow Model; a hybrid approach 68

$\begin{array}{lll}\mathbf{5 . 6} \text { Concluding remarks } & 70\end{array}$

$\begin{array}{lll}\text { CHAPTER } 6 \text { Conclusions } & 72\end{array}$

CHAPTER 7 Methodology 75

$\begin{array}{lll}7.1 & \text { Synthetic Methods } & 75\end{array}$

7.1.1 Equipment and Chemicals 75

$\begin{array}{lll}\text { 7.1.2 Compounds } & 75\end{array}$

7.1.2.1 5-bromo-2-(hexyloxy)benzaldehyde: G4-12s16 76

7.1.2.2 Hexyl 2-(hexyloxy)-5-nitrobenzoate: G5-047s10 77

7.1.2.3 (2-(hexyloxy)-5-nitrophenyl)(pyrrolidin-1-yl)methanone: G5-090s11 78

7.1.2.4 2-(hexyloxy)-5-nitrobenzaldehyde: G5-120s11 79

7.1.2.5 (5-bromo-2-(hexyloxy)phenyl)(phenyl)-methanol: G4-19s13 80

7.1.2.6 (5-(4-fluorophenoxy)-2-(hexyloxy)phenyl)-

7.1.2.7 -(phenyl)methanol: G4-74s14 83

7.1.2.8 (2-(hexyloxy)-5-(phenylamino)phenyl)-(phenyl)methanol: G2-100s9 86

7.1.2.9 (5-((4-fluorophenyl)amino)-2-(hexyloxy)-phenyl)(phenyl)methanol: G2-101s9 88

7.1.2.10 (5-((3-fluorophenyl)amino)-2-(hexyloxy)-phenyl)(phenyl)methanol: G2-102s9 90

7.1.2.11 Hexyl 2-(hexyloxy)-5-(phenylamino)-benzoate: G5-059s9

7.1.2.12 Hexyl 5-((4-fluorophenyl)amino)-2-(hexyloxy)benzoate: G5-060s9 95

7.1.2.13 Hexyl 5-((3-fluorophenyl)amino)-2-(hexyloxy)benzoate: G5-061s9 98

7.1.2.14 (2-(hexyloxy)-5-(phenylamino)phenyl)-(pyrrolidin-1-yl)methanone: G5-112s5 101 


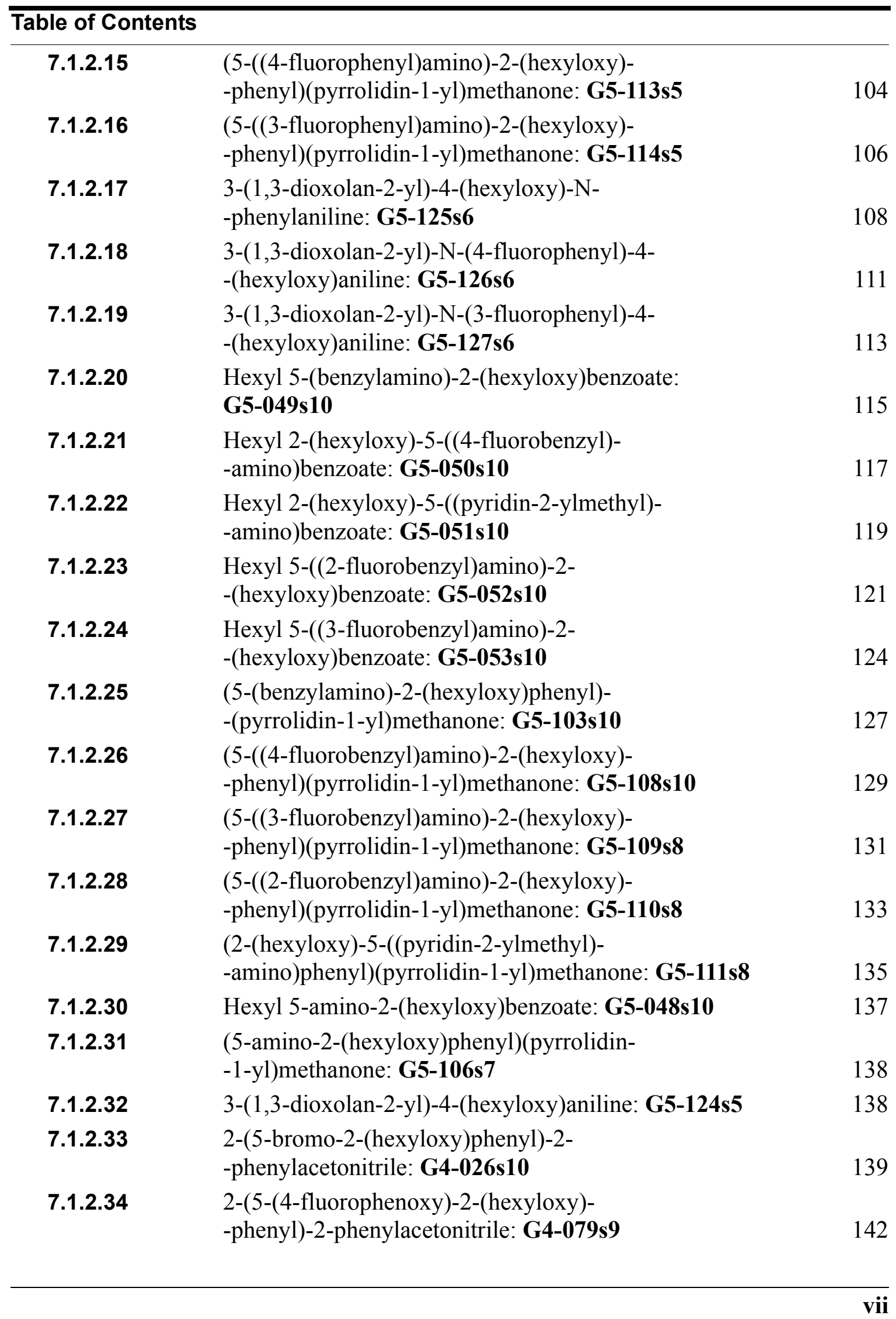


Table of Contents

7.1.2.35 2-(5-bromo-2-(hexyloxy)phenyl)-1,3-dioxolane: G1-130s5 145

7.1.2.36 2-(2-(hexyloxy)-5-nitrophenyl)-1,3-dioxolane: G5-122s6 145

7.1.2.37 2-(5-(4-fluorophenoxy)-2-(hexyloxy)-phenyl)-2-phenylethanamine hydrochloride:

ET-92 (G4-091s14 and G5-002s12)

7.1.2.38 (2-hydroxy-5-nitrophenyl)(pyrrolidin-1-

-yl)methanone: G5-098s6 and G5-088s8

7.1.2.39 5-(4-fluorophenoxy)-2-(hexyloxy)-

-benzaldehyde: G4-068s20 and G4-70s6 150

$\begin{array}{lll}7.2 & \text { Biological Assays: TEVC Methods } & 154\end{array}$

$\begin{array}{lll}\text { 7.2.1 In Vitro Transcription } & 154\end{array}$

$\begin{array}{ll}\text { 7.2.2 } & \text { Preparation and Microinjection of Oocytes }\end{array}$

$\begin{array}{lll}\text { 7.2.3 Whole Cell Recordings } & 156\end{array}$

$\begin{array}{lll}\text { 7.2.3.1 Compound Assays } & 156\end{array}$

$\begin{array}{ll}\text { References \& Notes } & 159\end{array}$

Appendix A NMR Spectra 166

A.1 5-bromo-2-(hexyloxy)benzaldehyde: G4-012s16 166

A.2 5-(4-fluorophenoxy)-2-(hexyloxy)benzaldehyde:

G4-068s20 \& G4-070s6

173

A.3 (5-(4-fluorophenoxy)-2-(hexyloxy)phenyl)-

-(phenyl)methanol: G4-074s14

181

A.4 2-(5-(4-fluorophenoxy)-2-(hexyloxy)phenyl)-2-phenylacetonitrile: G4-079s9

A.5 ET-92: 2-(5-(4-fluorophenoxy)-2-

-(hexyloxy)phenyl)-2-phenylethanamine

hydrochloride: G4-091s14

A.6 (2-hydroxy-5-nitrophenyl)(pyrrolidin-

-1-yl)methone: G5-088s8

205

A.7 (2-(hexyloxy)-5-nitrophenyl)(pyrrolidin-

-1-yl)methone: G5-090s10

212

A.8 (5-amino-2-(hexyloxy)phenyl)(pyrrolidin-

-1-yl)methone: G5-106s7

219

A.9 (5-((3-fluorobenzyl)amino)-2-(hexyloxy)-

-phenyl)(pyrrolidin-1-yl)methone: G5-109s8 
Table of Contents

Appendix B High Resolution Mass Spectra

226

B. 1

5-bromo-2-(hexyloxy)benzaldehyde: G4-012s16

226

B.2

5-(4-fluorophenoxy)-2-(hexyloxy)benzaldehyde:

G4-068s20 \& G4-070s6

B.3 (5-(4-fluorophenoxy)-2-(hexyloxy)phenyl)-(phenyl)methanol: G4-074s14

B.4

2-(5-(4-fluorophenoxy)-2-(hexyloxy)-phenyl)-2-phenylacetonitrile: G4-079s9

B.5 ET-92: 2-(5-(4-fluorophenoxy)-2-(hexyloxy)phenyl)-2-phenylethanamine hydrochloride: G4-091s14 


\section{List of Tables}

Table 7.1 High resolution mass spectrum (ESI) of 5-bromo-2(hexyloxy)benzaldehyde (G4-012s16)

Table 7.2 High resolution mass spectrum (ESI) of (5-bromo-2(hexyloxy)(phenyl))(phenyl)methanol (G4-019s13)

Table 7.3 High resolution mass spectrum (ESI) of 5(4-fluorophenoxy)-2-(hexyloxy)(phenyl)-methanol (G4-074s14)

Table 7.4 HPLC Conditions used to purify G4-068s16

Table 7.5 High resolution mass spectrum (ESI) of 5-

(4-fluorophenoxy)-2-(hexyloxy)benzaldehyde (G4-068s20) 


\section{List of Figures}

FIGURE 1.1 TAAR1 agonists. 2

FIGURE 1.2 The known signaling pathway of TAAR1.

FIGURE 1.3 Structures of ATP, cAMP, GTP and GDP. 5

FIGURE 1.4 In vitro methamphetamine stimulated TAAR1 dose response $\quad 8$

FIGURE 1.5 Structures of EPPTB and ET-92 (racemic) 9

FIGURE 1.6 Mouse in vivo studies of the effects of ET-92 or EPPTB in reducing Meth stimulated locomotion. 10

FIGURE 1.7 Racemic mixtures of some phenethylamine (PEA) analogs that bind to TAAR1. 11

FIGURE 1.8 Racemic mixtures of some cathinone analogs. 11

FIGURE 1.9 Structure of Guanabenz 12

FIGURE 1.10 Analogs of thyronamine. 12

FIGURE 1.11 TAAR1-specific compounds from Hoffmann-LaRoche. 12

FIGURE 2.1 The original ET-92 synthetic route. 15

FIGURE 2.2 The new synthetic route to ET-92. 16

FIGURE 2.3 The Miyaura boration reaction did not work with this $\begin{array}{ll}\text { substrate. } & 16\end{array}$

FIGURE 2.4 A boronic acid synthesis followed by a Chan-Lam reaction to make $\mathbf{1 5 .} 18$

FIGURE 2.5 A possible route to chiral ET-92. 20

FIGURE 2.6 Nitrilation reaction confirmed the feasibility of the new $\begin{array}{ll}\text { ET-92 route. } & 21\end{array}$

FIGURE 3.1 First new analog targets to be synthesized. 23

FIGURE 3.2 The first step in synthesizing compounds $\mathbf{1}$ and $\mathbf{2}$. 24

FIGURE 3.3 New targets and ET-92 25

FIGURE 3.4 The first steps towards the new analogs. 26

FIGURE 3.5 Reduction of ester to aldehyde. 26

FIGURE 3.6 Synthesis of a key intermediate. 27

FIGURE 3.7 Functionalizing the amine. 28

$\begin{array}{lll}\text { FIGURE } 3.8 \text { A failed Grignard reaction. } & 28\end{array}$ 
List of Figures

FIGURE 3.9 The final synthesizes of thyronamine analogs.

FIGURE 3.10 SAR comparison between (S)-sulpiride, ET-10, ET-92, and G5-109s8 through G5-114s5. 31

FIGURE 4.1 Compounds tested 32

FIGURE 4.2 In vitro methamphetamine stimulated TAAR1 concentration response $\quad 34$

FIGURE 4.3 Structure of Forskolin. 36

FIGURE 4.4 Pretreatment with $20 \mu \mathrm{M}$ G5-109s8 followed by $100 \mu \mathrm{M}$ Meth. $\quad 38$

FIGURE 4.5 Heads up competition between Meth and G5-109s8. 39

FIGURE 4.6 CFTR only control experiments to detect off-target effects. 41

FIGURE 4.7 Heads up competition between Meth and G5-110s8. 47

FIGURE 4.8 Heads up competition between Meth and G5-111s8. 47

FIGURE 4.9 Heads up competition between Meth and G5-112s5. 48

FIGURE 4.10 Heads up competition between Meth and G5-113s5. 48

FIGURE 4.11 Heads up competition between Meth and G5-114s5. 49

FIGURE 5.1 Kinetic Schemes for TAAR1. 54

FIGURE 5.2 The model is very sensitive to rate of equilibrium between $R_{i}$ and $R_{a}$. $\quad 57$

FIGURE 5.3 Simulation of competition between Meth and Inhibitor. 58

FIGURE 5.4 Receptor overexpression can lead to counter-intuitive $\begin{array}{ll}\text { results. } & 60\end{array}$

FIGURE 5.5 Oocyte response to various stimuli. 65

FIGURE 5.6 Slow inhibitor off-rate results in a total sub-basal $\begin{array}{ll}\text { response. } & 67\end{array}$

FIGURE 5.7 Very fast inhibitor on-rate deepens the trough. 67

FIGURE 5.8 Slow inhibitor on-rate prevents the complete inhibition $\begin{array}{ll}\text { of Meth. } & 68\end{array}$

FIGURE 5.9 Hybrid model allows trough-peak-trough response pattern. 70

FIGURE 7.1 Chromatographic purification of G4-019s9. 82

FIGURE 7.2 Chromatographic purification of G4-074s12. 85

FIGURE 7.3 Chromatographic purification of G2-100s8. 87

FIGURE 7.4 Chromatographic purification of G2-101s8. 89

FIGURE 7.5 Chromatographic purification of G2-102s8. 91

FIGURE 7.6 Chromatographic purification of G5-059s7. 93

FIGURE 7.7 Chromatographic purification of G5-060s7. 97 
FIGURE 7.8

Chromatographic

purification of G5-061s7.

100

FIGURE 7.9 Chromatographic purification of G5-112s4. 103

FIGURE 7.10 Chromatographic purification of G5-113s4. 105

FIGURE 7.11 Chromatographic purification of G5-114s4. 107

FIGURE 7.12 Chromatographic purification of G5-125s5. 110

FIGURE 7.13 Chromatographic purification of G5-126s5. 112

FIGURE 7.14 Chromatographic purification of G5-127s5. 114

FIGURE 7.15 Chromatographic purification of G5-049s7. 116

FIGURE 7.16 Chromatographic purification of G5-050s7. 118

$\begin{array}{lll}\text { FIGURE 7.17 Chromatographic purification of G5-051s7. } & 120\end{array}$

FIGURE 7.18 Chromatographic purification of G5-052s7. 123

FIGURE 7.19 Chromatographic purification of G5-053s7. 126

FIGURE 7.20 Chromatographic purification of G5-103s8. 128

FIGURE 7.21 Chromatographic purification of G5-108s7. 130

FIGURE 7.22 Chromatographic purification of G5-109s7. 132

FIGURE 7.23 Chromatographic purification of G5-110s7. 134

FIGURE 7.24 Chromatographic purification of G5-111s7. 136

FIGURE 7.25 Chromatographic purification of G4-026s8. 141

FIGURE 7.26 Chromatographic purification of G4-079s8. 144

FIGURE 7.27 Chromatographic purification of G5-122s5. 147

FIGURE 7.28 Chromatographic purification of G4-068s14. 152 
For the past $30^{+}$years, the paradigm of methamphetamine's (Meth) mechanism of action (MOA) ${ }^{1}$ has been that it blocks the uptake of extracellular dopamine (DA) and interferes with the vesicular monoamine transporters (VMAT) causing an increase in intracellular (cytosol) DA. Once intracellular levels of DA are high enough, the DA gradient-dependent dopamine transporter (DAT) allows the DA to move out of the neuron. Meth effects the release of serotonin and norepinephrin in similar ways. Due to dopamine's ${ }^{2,3}$ association with pleasure, particularly the anticipation of pleasure, the dopamine hypothesis of addiction has been the basis for trying to develop treatments for Meth addiction. There is a membrane-bounded receptor, the Trace Amine Associated Receptor 1 (TAAR1), which is activated by Meth, that is a new potential target for treating Meth addiction. ${ }^{4,5}$

\section{$1.1 \quad$ Background on Trace Amine Associated Receptor 1 (TAAR1)}

A good review of the current TAAR1 literature was written by Gregory Miller. ${ }^{6}$ TAAR1 was discovered in $2001^{4,7}$ and is a G-protein coupled receptor (GPCR) widely distributed throughout the body and is activated by a variety of substrates (FIGURE 1.1) including the neurotransmitter dopamine (DA); the biogenic trace amines: $\beta$-phenylethylamine (PEA), p-tyramine, octopamine, synephrine, and 


\section{Background on Trace Amine Associated Receptor 1 (TAAR1)}

thyronamines. TAAR1 is also affected by exogenous drugs of abuse (FIGURE 1.1),

such as: amphetamine, N-methamphetamine (Meth) and methylenedioxymethamphetamine (MDMA, AKA: Ecstasy). ${ }^{4}$ Of particular importance for this work, TAAR1 is co-expressed with the dopamine transporter (DAT) and the dopamine receptors as part of the dopaminergic system in the ventral tegmental area (VTA), which is a major part of the brain's reward circuitry. As part of the dopaminergic system, TAAR1 is suspected to be involved in depression, addiction attention-deficit/hyperactivity disorder, Parkinson's disease, schizophrenia, ${ }^{8}$ and anxiety. ${ }^{9-11}$ There is also evidence that links TAAR1 to fibromyalgia ${ }^{12}$ and B-cell pathologies (lymphomas). ${ }^{13}$ TAAR1 is largely expressed on intracellular membranes. $4,6,8-11,14$

FIGURE 1.1: TAAR1 agonists.

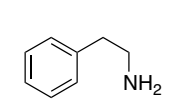

$\beta$-phenethylamine (PEA)

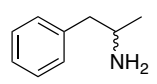

Amphetamine

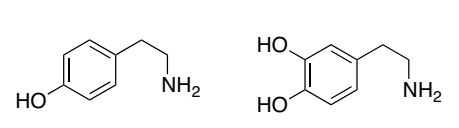

p-Tyramine

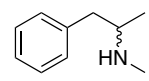

Methamphetamine (Meth)

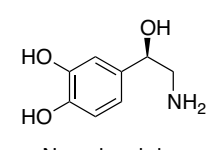

Norepinephrine

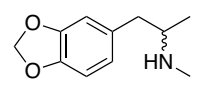

Methylenedioxymethamphetamine (MDMA)

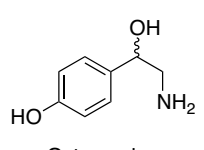

Octopamine

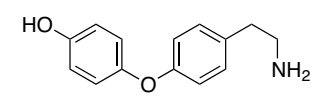

Thyronamine

FIGURE 1.2 shows the sequence of the TAAR1 signaling pathway starting with Meth binding to the TAAR1- $\mathrm{G}_{\alpha \mathrm{s}} / \mathrm{G}_{\beta / \gamma}$ complex (1). Once activated by Meth, TAAR1 stimulates $\mathrm{G}_{\alpha \mathrm{s}}$ to exchange GDP ${ }^{15,16}$ for GTP (2). The TAAR1- $\mathrm{G}_{\alpha \mathrm{s}} / \mathrm{G}_{\beta / \gamma}$ complex disassociates, and $\mathrm{G}_{\alpha \mathrm{s}}$ binds to adenylyl cyclase (3). At this stage, the $\mathrm{G}_{\beta / \gamma}$ subunit could modulate the activity of effectors (enzymes, ion channels, etc). 


\section{Background on Trace Amine Associated Receptor 1 (TAAR1)}

In step 4, adenylyl cyclase converts ATP to cAMP. From that point, signaling proceeds through the $\mathrm{cAMP} /$ protein kinase A (PKA)/cAMP response element binding protein $(\mathrm{CREB})$ pathway. ${ }^{10}$ Also, activated TAAR1 signals through the protein kinase $\mathrm{C}(\mathrm{PKC}) / \mathrm{Ca}^{+2} /$ nuclear factor of activated T-cells (NFAT) pathway. ${ }^{10}$ The literature clearly indicates a connection between TAAR1 and the NFAT pathway, however the precise mechanism by which they are connected was not proposed. One possible way for them to be connected is through a $\mathrm{Ca}^{+2}$ cyclic nucleotidegated ion channel (CNG). Both the CREB and NFAT pathways lead to the blocking of monoamine uptake.

There is evidence that TAAR 1 tonically activates inwardly rectifying $\mathrm{K}^{+}$channels, presumably by Kir3-type $\mathrm{K}^{+}$channels via activated $\mathrm{G}_{\beta / \gamma}$. TAAR1 inhibition increases affinity of dopamine to the $\mathrm{D}_{2}$-receptor $\left(\mathrm{D}_{2} \mathrm{R}\right)$ and reduces the desensitization rate of $\mathrm{D}_{2} \mathrm{R} .{ }^{11}$ Furthermore, TAAR1- $\mathrm{D}_{2} \mathrm{R}$ heterodimers have been observed. ${ }^{14}$ Early work with the thyronamine family of compounds, including their analogs developed by Dr. Edwin Tan, ${ }^{17,18}$ implicated TAAR1 in the thermoregulatory processes, but that has since been challenged. ${ }^{10,19}$ Basal (constitutive) or tonic activity of TAAR1 has been hypothesized ${ }^{9,11}$ and research in this area is on going. 


\section{Background on Trace Amine Associated Receptor 1 (TAAR1)}

FIGURE 1.2: The known signaling pathway of TAAR1. (1) System at rest until Meth binds to TAAR1. (2) Activated TAAR1 stimulates $\mathrm{G}_{\alpha \mathrm{s}}$ to exchange GDP for GTP. GDP is then recycled into GTP by nucleoside diphosphate kinase. The literature has little information about other effects this GDP might have. (3) The TAAR $1-G_{\alpha s} / G_{\beta / \gamma}$ complex disassociates and $G_{\alpha s}$ binds to adenylyl cyclase (AC). (4) AC converts ATP to cAMP. It is then possible that cAMP activates the PKC/NFAT regulatory pathway via a calcium ion cyclic nucleotide-gated ion channel. cAMP also activates the CREB regulatory pathway via PKA. Additionally, the cAMP produced by the TAAR1 pathway may affect other pathways. Also, TAAR1 releases the Meth (shown in step 4 but could happen in step 3). (5) $\mathrm{G}_{\alpha \mathrm{s}}$ disassociates from AC, and GTP is hydrolyzed to GDP. The system is now ready to reset. Original artwork by Troy Wahl.

1

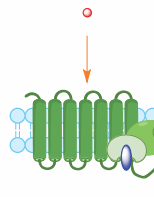

2

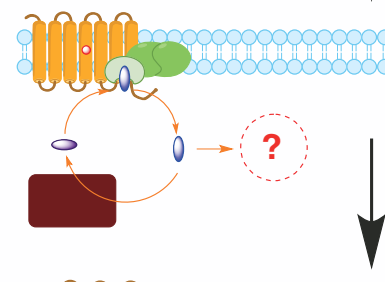

3

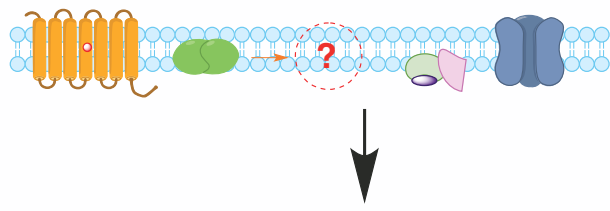

4
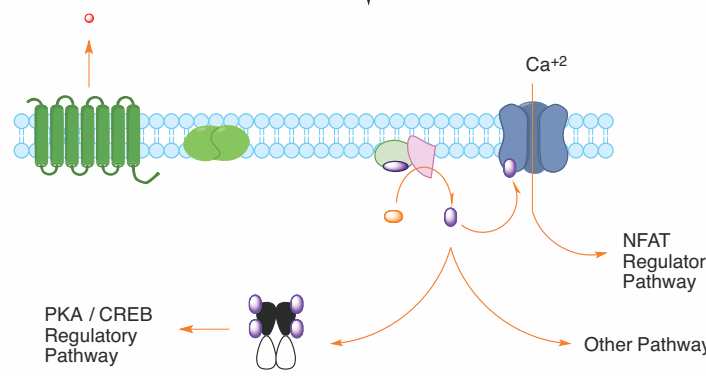

$$
\text { Pathway }
$$

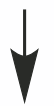

5

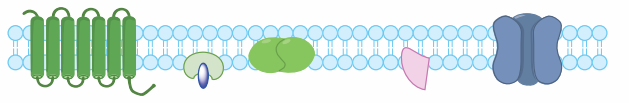

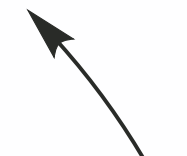
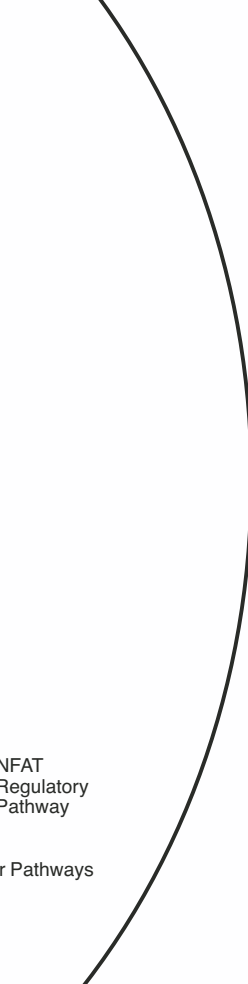

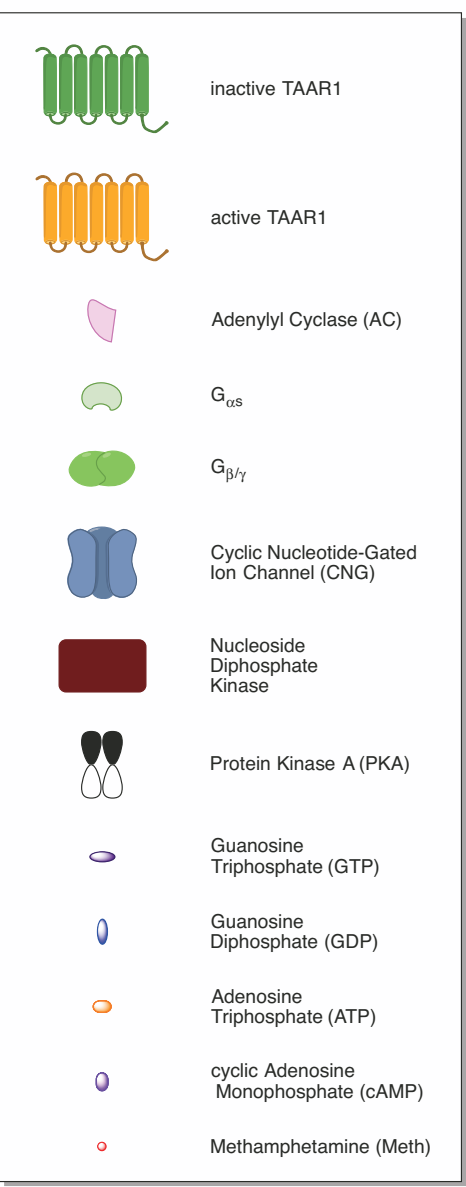




\section{Methamphetamine}

FIGURE 1.3: Structures of ATP, cAMP, GTP and GDP.

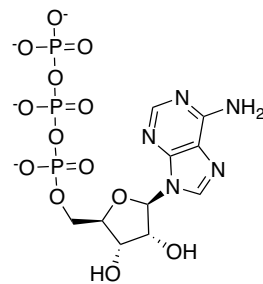

adenosine triphosphate (ATP)

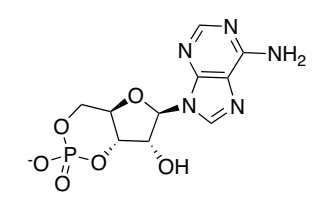

cyclic adenosine monophosphate (cAMP)

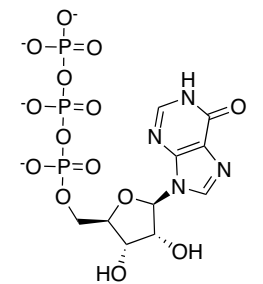

guanosine triphosphate (GTP)

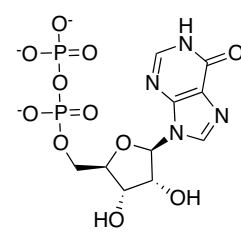

guanosine diphosphate (GDP)

\section{$1.2 \quad$ Methamphetamine}

Methamphetamine (Meth), FIGURE 1.1, is a psychostimulant that effects the Dopamine Transporter (DAT), Norepinephrine Transporter (NET), Serotonin Transporter (SERT), Vesicular Monoamine Transporters (VMAT) ${ }^{20,21}$ and TAAR $1 .{ }^{4}$ By inhibiting VMAT, Meth causes DA to accumulate in the cytosol until the concentration of DA is high enough that gradient dependent DAT allows a net flow of DA out of the neuron and into the synaptic cleft. In the parlance of chemical biology, Meth blocks the re-uptake of neurotransmitters and thus elevates signaling. Meth affects norepinephrine and serotonin in a similar manner as DA.

Dopamine $^{3}$ is part of the reward system and is associated with pleasant or excited feelings, which in part, is why it is implicated in addiction. DA is considered to modulate prediction error, ${ }^{2}$ which is the difference between an expected outcome and the actual outcome of an event. When an expectation is met, dopamine levels increase, reinforcing the expectation/lesson, otherwise dopamine levels drop. DA, also, is associated with seeking behaviors. ${ }^{22-27}$ In general, medications that increase DA in the synaptic cleft, such as amphetamine and meth- 


\section{Methamphetamine}

ylphenidate, ${ }^{28}$ are effective attention deficit hyperactive disorder (ADHD) medications. Finally, DA is associated with agitation and anxiety (worry/anxiety can be described, in part, as the expectation of negative or undesirable outcomes). ${ }^{29}$

Norepinephrine ${ }^{30}$ is part of the fight-or-flight response (adrenergic system) and increases heart rate, blood pressure and the release of glucose into the blood. Norepinephrine is also involved in attention, wakefulness (arousal) and alertness. Atomoxetine (Strattera) ${ }^{31}$ is a selective norepinephrine reuptake inhibitor $\left(4 \mathrm{nM} \mathrm{K}_{\mathrm{i}}\right)$ prescribed to treat ADHD, it is also weak serotonin reuptake inhibitor $(77 \mathrm{nM} \mathrm{K}$ ). Both epinephrine (adrenaline) and norepinephrine are associated with the panic symptoms of anxiety.

Serotonin $^{32}$ is involved in mood regulation with low levels being associated with depression. Extremely high levels of serotonin results in serotonin syndrome ${ }^{33}$ which is potentially fatal.

Overall Meth, like all the amphetamines, increases alertness, wakefulness, and attention (particularly to repetitive tasks) but decreases appetite and is a euphoric. Side-effects include addiction, high blood pressure, irritability, aggression, hyperthermia and anxiety-like symptoms (especially paranoia and obsessive-compulsive disorder). Due to its benefits, Meth might appeal to those seeking to reduce stress due to fatigue, those with excessive time demands (long work hours or multiple 


\section{Justification for targeting TAAR1}

jobs or overbooked social commitments), excessive deadlines, who need to focus on highly repetitive tasks, who are bored, depression or who perceive the needed to get a competitive edge over others.

\subsection{Justification for targeting TAAR1}

TAAR1 was chosen as the target receptor for treating methamphetamine (Meth) addiction because TAAR1 is activated by Meth in a dose (concentration * 1.5 minutes * $4 \pm 1 \mathrm{~mL} /$ minute) dependent manner (FIGURE 1.4A). In an experiment with an oocyte that was not injected with hTAAR1 encoding RNA, Meth caused a drop in transmembrane conductance (FIGURE 1.4B, red shaded region). While this small drop in transmembrane conductance, which has been observed only once, needs to be verified before considered as solid evidence for an new Meth target, it is clearly different than the response to Meth when TAAR1 is present. Also, in vivo mouse studies, FIGURE 1.6, show how the dose-dependent inhibition of TAAR1 by ET-92 ${ }^{17}$ and EPPTB $^{34}$ (FIGURE 1.5) reduces Meth stimulated locomotion. These results represent the strongest type of justification for a medicinal chemistry project, namely a desired phenotypical response (blocking the effects of Meth) due to the action of a known molecular target (TAAR1). 


\section{Justification for targeting TAAR1}

FIGURE 1.4: A) Two electrode voltage clamp (TEVC) methamphetamine dose (concentration * 1.5 minutes * 4 $41 \mathrm{~mL} /$ minute) response from Xenopus Laevis frog oocyte injected with hTAAR1 and hCFTR encoding RNA $(n=1)$. The doses, indicated on the graph, of Meth in Frog Ringer's solution (red bars) were pulsed for 1.5 minutes. At all other times the oocyte was washed with Frog Ringer's solution. The experiment $(\mathrm{n}=1)$ was done by Dr. Yohei Noramatsu, OHSU. The red line is an approximate baseline. B) TEVC Meth response in an oocyte injected with hCFTR encoding RNA. Meth $(10 \mu \mathrm{M}$, red region) in Frog Ringer's solution was pulsed for 1.5 minutes followed by washing with Frog Ringer's solution $(n=1)$. The horizontal lines are visual aids.
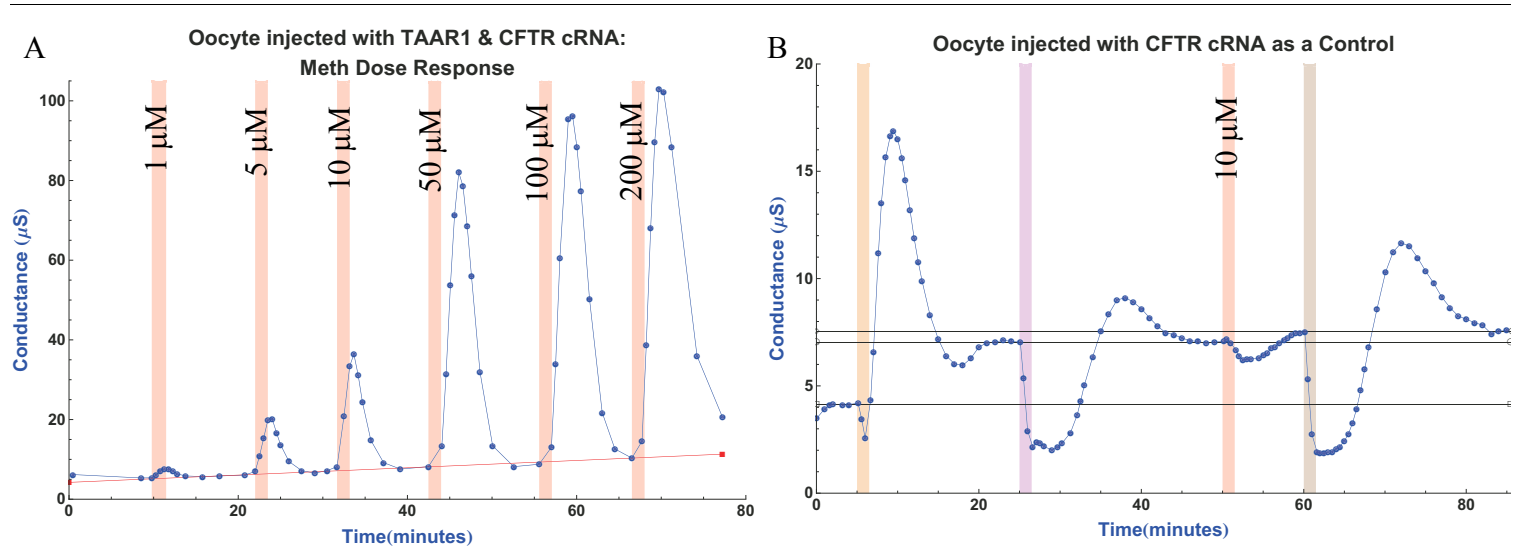

In FIGURE 1.4, the two electrode voltage clamp (TEVC) results are shown from dosing frog oocytes injected with hTAAR1 and hCFTR encoding RNA (FIGURE 1.4A) or just hCFTR encoding RNA (FIGURE 1.4B) with Meth, both with $\mathrm{n}=1$. CFTR (cystic fibrosis transmembrane conductance regulator) ${ }^{35}$ is a chloride channel that is activated by cAMP. By pairing TAAR1, whose activation results in increased cAMP production, and CFTR in an oocyte, a biological circuit is made for assaying the activity of TAAR1 via monitoring the oocytes transmembrane conductance. An oocyte just injected with hCFTR encoding RNA served as the control experiment. In the absence of TAAR1, Meth caused a drop in transmembrane conductance in this single run. It is unlikely that the drop was caused by the effects of Meth on any of the dopamine, norepinephrine, serotonin receptors or transporters as the oocytes do not express them. The drop in transmembrane con- 


\section{Justification for targeting TAAR1}

ductance might involve a hitherto unknown target of Meth as a literature survey

has not revealed any such reports. Confirmation of this result will be needed.

\section{FIGURE 1.5: Structures of EPPTB and ET-92 (racemic)}

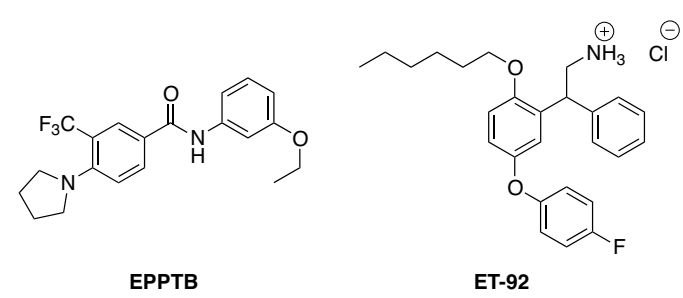

The in vivo results for ET-92, FIGURE 1.6A-C, were known at the start of the project but those of EPPTB, FIGURE 1.7D-F, were not as EPPTB was still fairly new. ${ }^{11}$ Both of these compounds, which were designed to be TAAR1 antagonists, reduce Meth stimulated locomotion in mice in a dose-dependent manner thus validating that TAAR1 plays a significant role in Meth signaling and potentially a target for treating Meth addiction. Meth self-administration experiments with EPPTB or ET-92 treatments have yet to be done. Such experiments would demonstrate whether or not TAAR1 antagonists modulate the motivation to acquire or selfadminister Meth. However, their lack does not diminish the potential of treating Meth addiction by targeting TAAR1 as self-administration experiments, and other animal model testing, are usually done fairly late in pre-clinical development. Animal efficacy testing late in the project development may seem counter-intuitive but the expense of animal testing is great enough that a viable drug-candidate (potent, bioavailable, low toxicity, etc.) is desired before proceeding to test efficacy. 


\section{Justification for targeting TAAR1}

FIGURE 1.6: Mouse in vivo studies of the effects of ET-92 (A-C) or EPPTB (D-F) in reducing Meth stimulated locomotion. From these studies it is clear that both ET-92 and EPPTB inhibit Meth stimulated locomotion in a dose-dependent manner. The thick lines are cubic polynomial least-squares models of the data, with the exception of the model for the acclimation data which is a linear least-squares model. The shaded regions are the model lines' 95\% confidence intervals for their respected model. The modeling provides a very quick check for statistical difference - the less overlap between the confidence intervals, the greater the probability that the data sets are statistically different. In all cases, the vehicle was DMSO. Data was provided by Madeline Grandy, Ashley Kimbell, and Katie Tallman and the statistical modeling was done by Troy Wahl. ${ }^{36}$
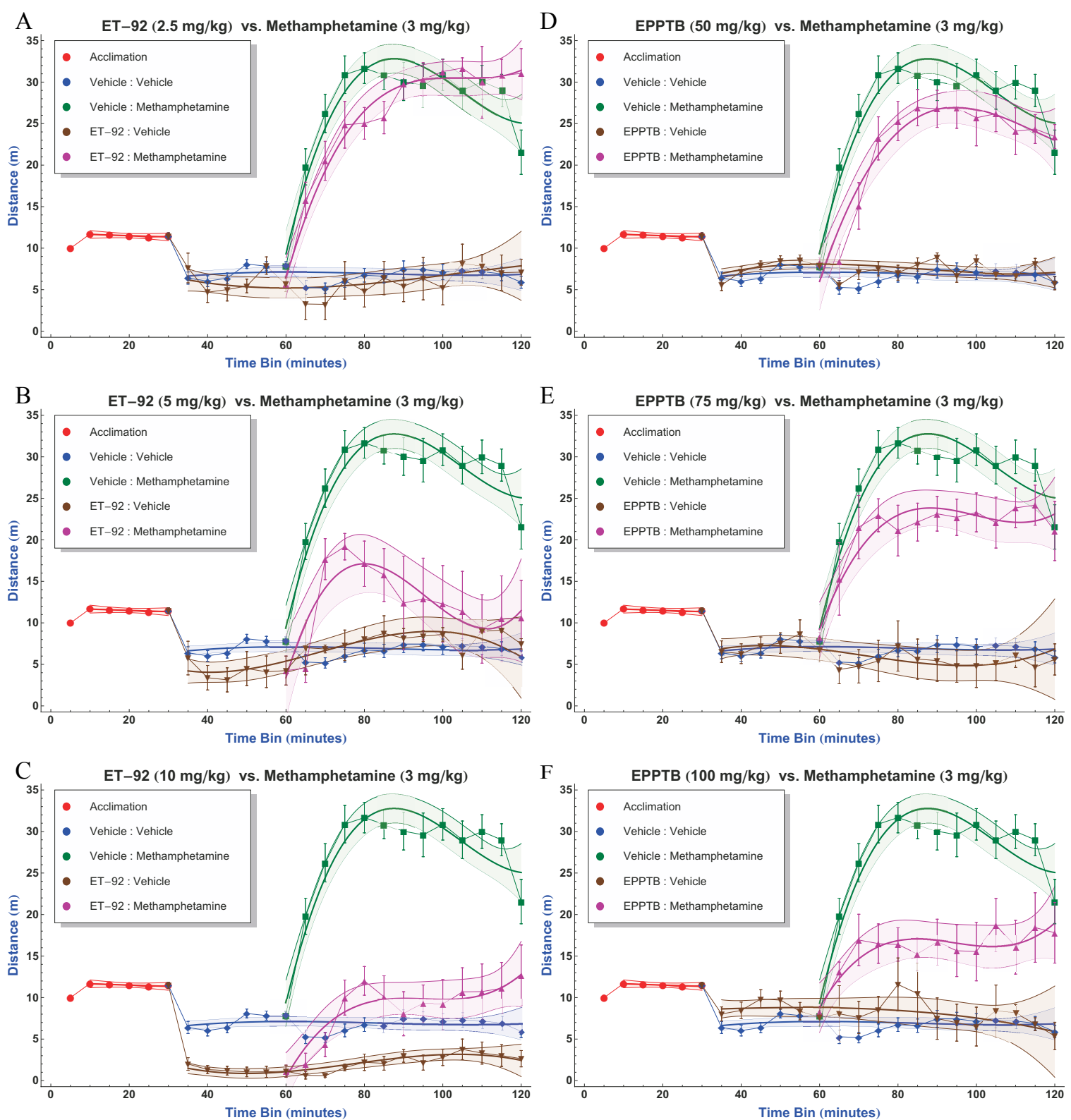


\section{Overview of Current TAAR1 Medicinal Chemistry}

\subsection{Overview of Current TAAR1 Medicinal Chemistry}

TAAR1 binds a variety of biogenic trace amines, as have been previously listed (FIGURE 1.1), ${ }^{5,6}$ it also binds a wide range of synthetic analogs of phenethylamine (PEA), some of which are shown in FIGURE 1.7. However Simmler ${ }^{20}$ reported that cathinones ( $\beta$-keto amphetamines, FIGURE 1.8) have poor binding affinities to TAAR1. Guanabenz, FIGURE 1.9, an antihypertensive $\alpha_{2}$ adrenergic agonist is also a highly potent TAAR 1 agonist. ${ }^{14}$ The first synthetic TAAR1 ligands were those of $\operatorname{Tan}^{17}$ and are analogs of thyronamine, FIGURE 1.10. The problem with all of these compounds, as indicated in the above citations, is that they lack TAAR1 specificity, except perhaps the thyronamines. The lack of specificity is not necessarily a problem for a drug so long as side-effects are minimal. However, the lack of specificity becomes a major problem for the development of tool kits to study TAAR1 in vivo because side-effects can confound the interpretation of experimental results.

FIGURE 1.7: Racemic mixtures of some phenethylamine (PEA) analogs that bind to TAAR1.
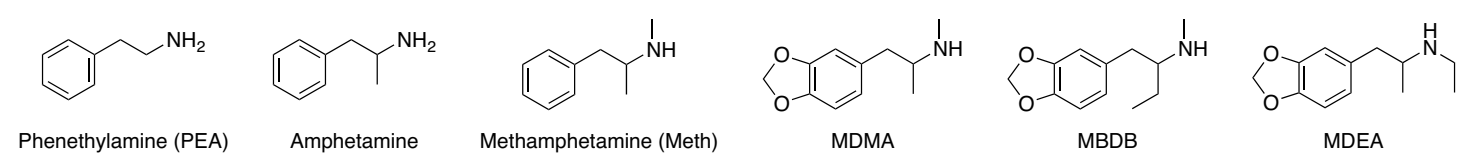

FIGURE 1.8: Racemic mixtures of some cathinone analogs.
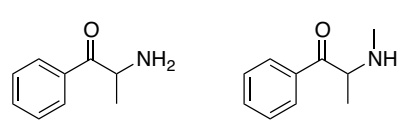

Cathinone

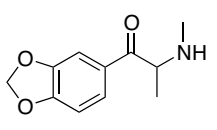

Methylone
Methcathinone

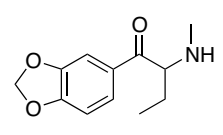

Butylone

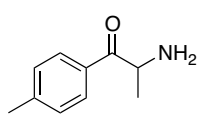

Mephedrone

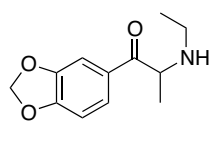

Ethylone

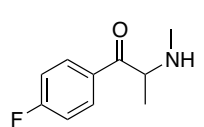

Flephedrone

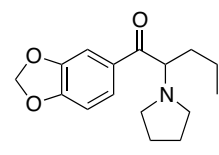

MDPV

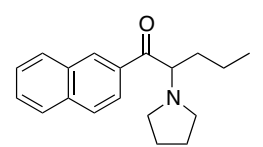

Naphyrone

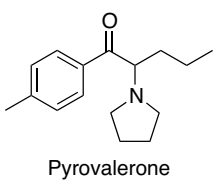




\section{Overview of Current TAAR1 Medicinal Chemistry}

FIGURE 1.9: Guanabenz

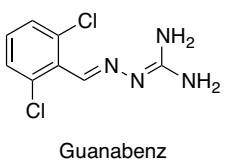

FIGURE 1.10: Analogs of thyronamine. ET-13 is a TAAR1 agonist, while ET-92 is an antagonist. ET-13 and ET-92 are racemic mixtures.

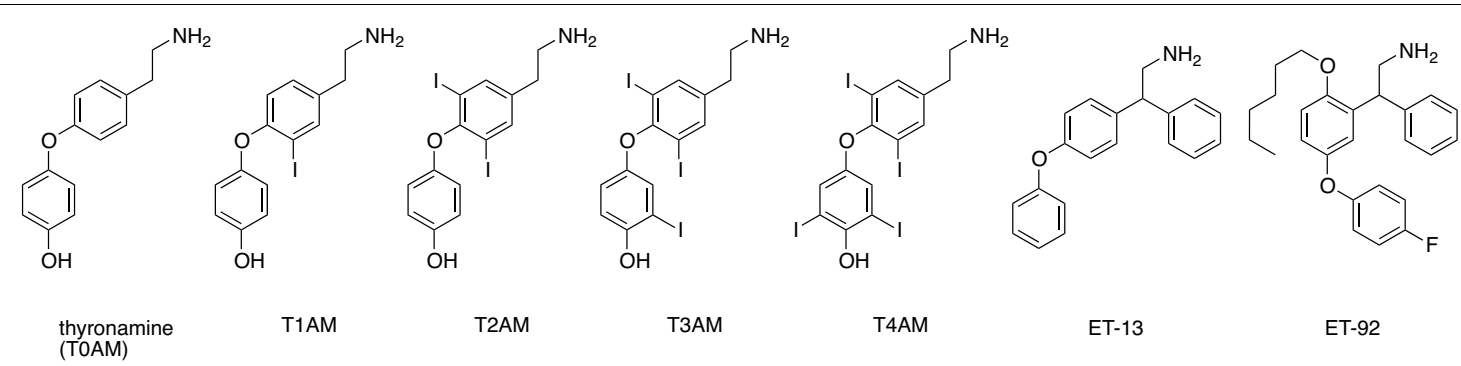

Currently, the only documented TAAR1 specific compounds are from Hoffmann-LaRoche, FIGURE 1.11. These compounds range from antagonist, EPPTB, to partial agonists, RO5203648, and full agonists, RO5073012 and RO5166017..$^{9,10,34,37}$ Selectivity of these compounds were determined by screening against over 100 receptors, ion channels, transporters and enzymes, with the most problematic off-target being the $\alpha_{2}$-adrenergic receptor. In the course of developing these compounds Hoffmann-LaRoche created an extensive patent library, ${ }^{8,34,38-56}$ which greatly restricts the development of new compounds targeting TAAR1.

FIGURE 1.11: TAAR1-specific compounds from Hoffmann-LaRoche.<smiles>CCOc1cccc(NC(=O)c2ccc(N3CCCC3)c(C(F)(F)F)c2)c1</smiles>

EPPTB

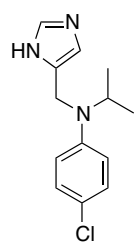

RO5073012

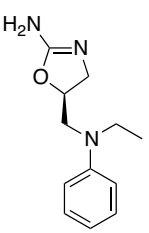

RO5166017

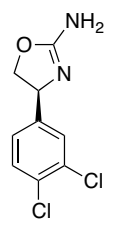

RO5203648

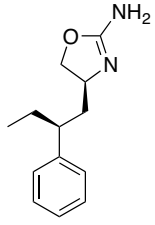

RO5256390

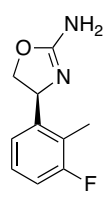

RO5263397 


\section{Overview of Current TAAR1 Medicinal Chemistry}

As seen in FIGURE 4.6, ET-92 is more efficacious in vivo in mice than EPPTB

by a molar ratio greater than 10.7:1, which made it an attractive lead for further development. Furthermore, Oregon Health \& Science University (OHSU) owns the patent on the thyronamine family, ${ }^{57}$ and there is substantial room for further

SAR (structure-activity-relationship) development in Tan's work. ${ }^{17}$ At the outset of the project, the thyronamines, ET-92 in particular, had 3 liabilities: poor druglike properties (high lipophilicity, poor solubility and a hydroquinone core, which has known toxicity issues), thermoregulatory side-effects and a lengthy synthetic route. 


\subsection{Cost-effective synthetic route to ET-92 and its analogs}

The original synthesis of ET-92, ${ }^{17}$ FIGURE 2.1, is fourteen steps starting from 2,5-dihydroxybenzaldehyde with an overall yield of $15.3 \%$, and involves multiple protecting groups with steps $\mathbf{4} \rightarrow \mathbf{5}$ and $\mathbf{9} \rightarrow \mathbf{1 0}$ taking up to 7 days each to complete. In order to develop ET-92, or closely related analogs, into an FDA approved drug, large batches of cGMP grade material would need to be produced. Based on an old estimate of $\$ 10,000$ per day ${ }^{58}$ in a process plant for facility charges, that might not include the costs of setting-up or clean-up, original ET-92 synthesis would be expensive (minimum facility charges: $\$ 150 \mathrm{~K}$ - $\$ 410 \mathrm{~K}$ ) even before the cost of reagents and waste disposal are considered. Finally, the length of the synthesis would make rapid develop of ET-92 analogs difficult. For these reasons, a new cost-effective synthetic route was developed, FIGURE 2.2. 


\section{Cost-effective synthetic route to ET-92 and its analogs}

FIGURE 2.1: The original ET-92 synthetic route ${ }^{17}$ starting with 2,5-dihydroxybenzaldehyde (1) or with the alternative starting material, methyl 2,5-dihydroxybenzoate $\left(\alpha_{1}\right)$, involves fourteen steps from 1 with an overall yield of $15.3 \%$.<smiles>COC(=O)c1cc(O)ccc1OCCO</smiles>

$\alpha 1$

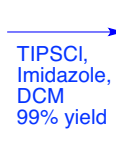

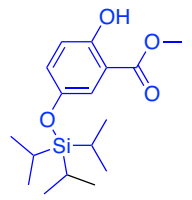

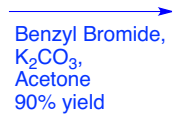

$90 \%$ yield

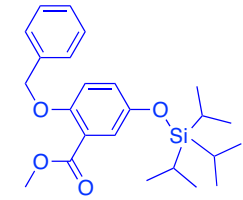

$\alpha 3$

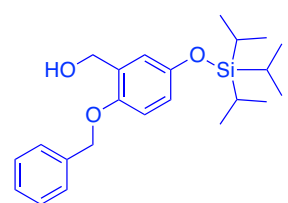

$\alpha 4$

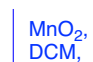

97\% yield
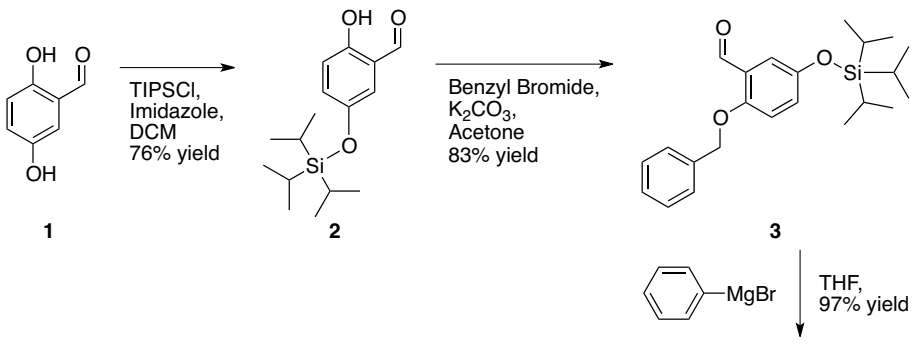

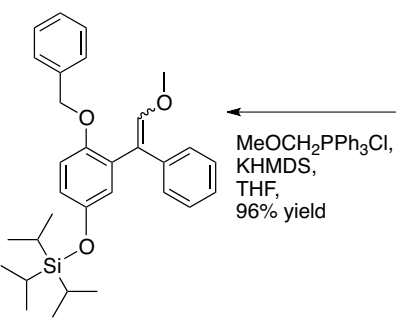

6

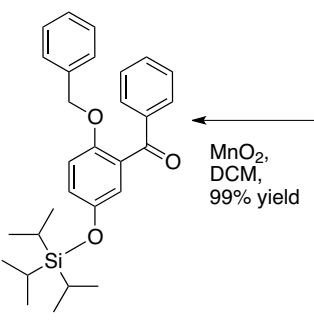

5

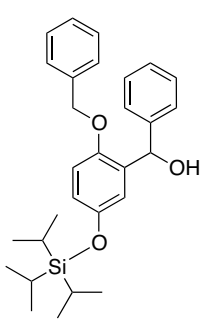

4
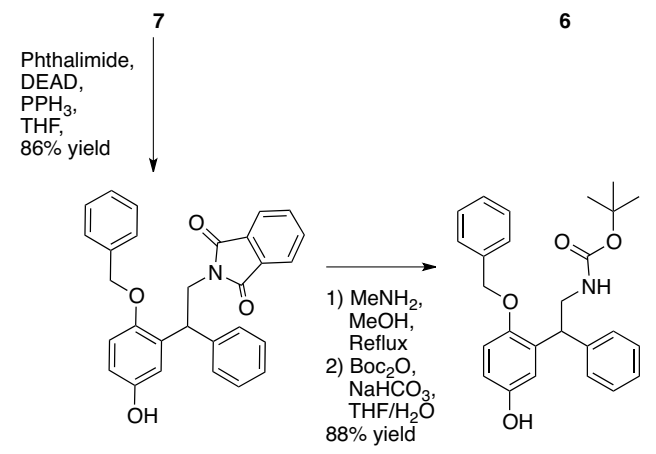

8

Overall yield: $15.3 \%$ from 1 Number of Steps: 14

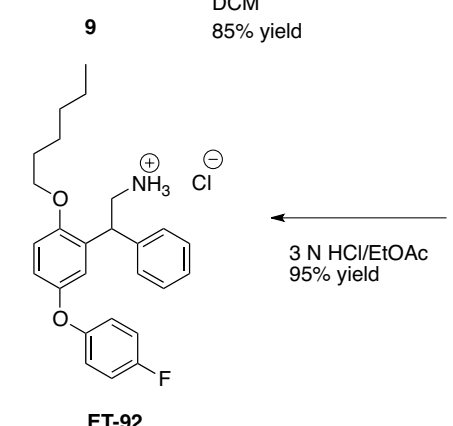

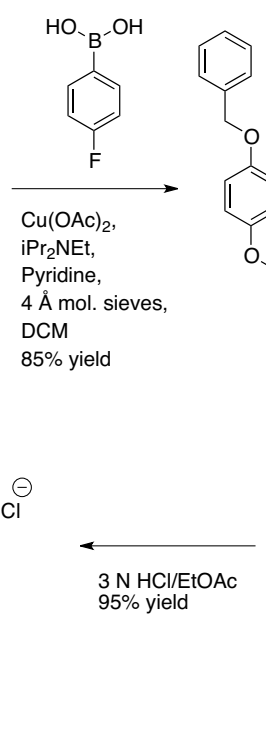

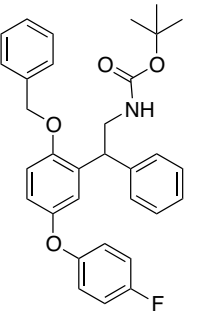

10
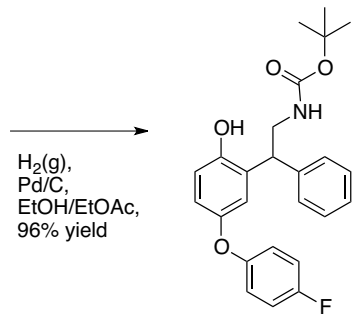

11

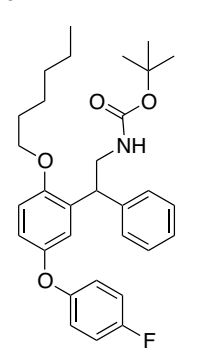

12 


\section{Cost-effective synthetic route to ET-92 and its analogs}

FIGURE 2.2: The new synthetic route to ET-92. Most steps are complete in less than 4 hours and are in high yield. Further optimization of $\mathbf{1 4} \rightarrow \mathbf{1 5}$ and $\mathbf{1 7} \rightarrow$ ET-92 is needed as well as improved purification (likely via distillation).

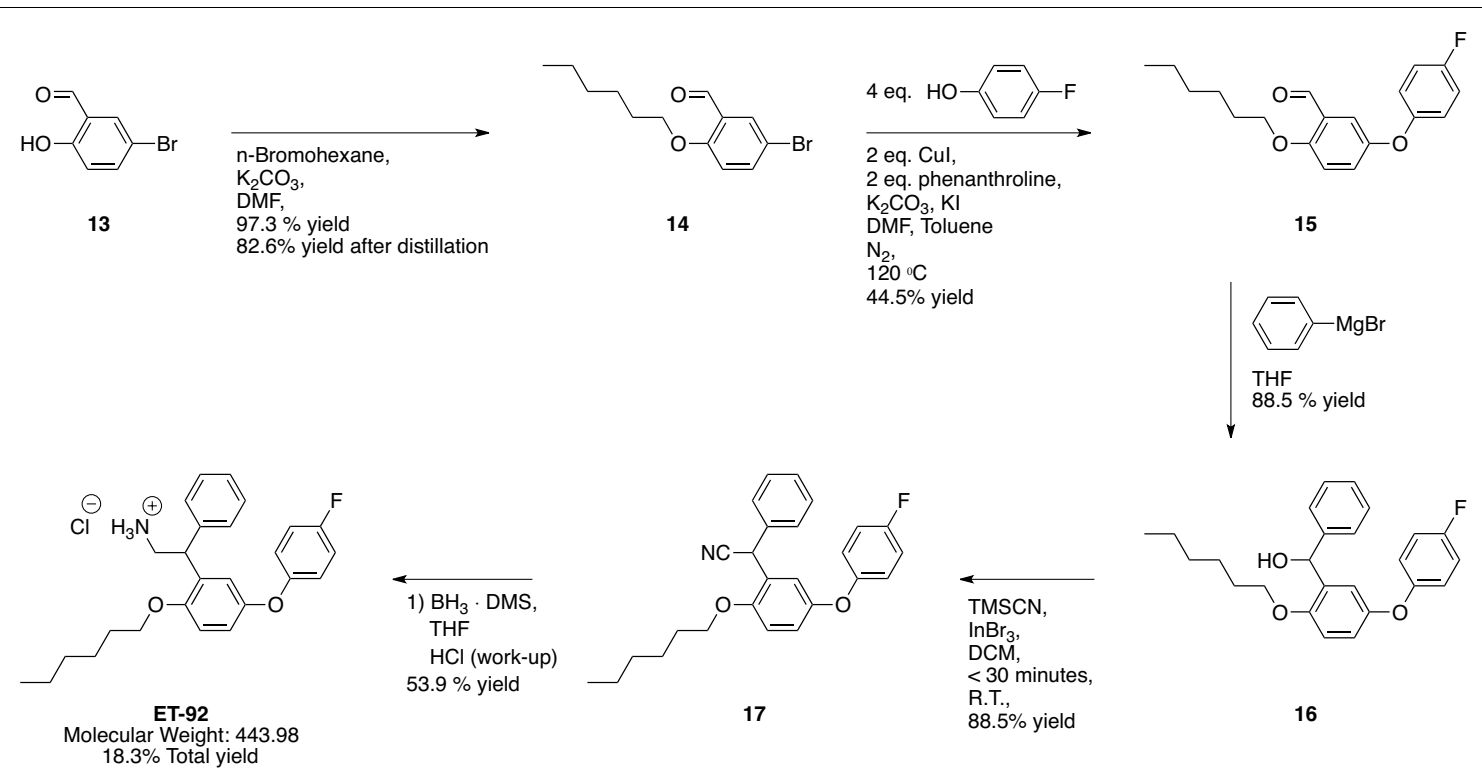

A new 5 step synthetic route was developed for ET-92 starting with the alkylation of 5-bromosalicylaldehyde, $\mathbf{1 3} \rightarrow \mathbf{1 4}$, which is quantitatively complete within 4 hours and resulting in a $97.3 \%$ isolated yield. In order to produce a publication quality NMR, 14 was vacuum distilled (46 mTorr, bp $104-130{ }^{\circ} \mathrm{C}$ at 46 mTorr, $85.6 \%$ total yield). However, distillation at this step is not necessary as the crude product is pure enough to use in the next reaction.

FIGURE 2.3: The Miyaura boration reaction did not work with this substrate.
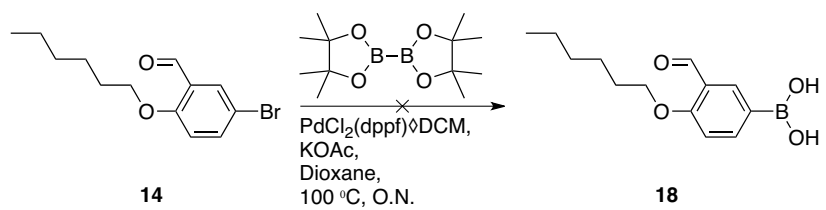

18

To convert 14 into 15, several different reaction types were tried: Miyaura

boration (FIGURE 2.3) $)^{59-66}$ followed by Chan-Lam reaction $(\mathbf{2 0} \rightarrow \mathbf{1 5}$ in FIGURE 


\section{Cost-effective synthetic route to ET-92 and its analogs}

2.4), a traditional boronic acid synthesis followed by Cham-Lam reaction (FIG-

URE 2.4) and finally the modified Ullmann reaction (14 $\rightarrow \mathbf{1 5}$ in FIGURE 2.2).

The use of copper catalyzed reactions (Chan-Lam and Ullmann) was preferred to palladium catalyzed reactions (Miyaura and Buchwald-Hartwig) for economic reasons, copper catalysts being much less expensive than palladium ones. If the Miyaura boration had worked, then there was the possibility of immediately following it with a Buchwald-Hartwig reaction to synthesis $\mathbf{1 5}$ in a one-pot reaction. It was for that reason that the Miyaura boration reaction was tried first. The Miyaura boration also served as an indicator for other palladium catalyzed reactions, failure of this reaction lead to the conclusion not to pursue other palladium catalyzed reactions. Although the Miyauara boration is known to work with 5-

bromo-2-(methoxymethoxy)benzoic acid, ${ }^{63}$ 5-bromo-2-(methoxymethoxy)benzaldehyde ${ }^{67}$ and 5-bromosalicylaldehyde under microwave reaction conditions, ${ }^{68}$ it produced no detectable product using $\mathbf{1 4}$ and $\mathrm{PdCl}_{2}(\mathrm{dppf}) \cdot \mathrm{DCM}$ as the catalyst.

In the more traditional boronic acid synthesis $(\mathbf{1 9} \rightarrow \mathbf{2 0}$ in FIGURE 2.4) two issues were encountered: the acetal (19) appears to coordinate water, which in turn hydrolyzes the acetal over time; also, the boronic acid tended to form mixed anhydrides which did not react well in the Chan-Lam step. This is a stark contrast to the results achieved in previous work at Celera Genomics with 2-benzyloxy-3-bromobenzaldehyde. 


\section{Cost-effective synthetic route to ET-92 and its analogs}

FIGURE 2.4: A traditional boronic acid synthesis followed by a Chan-Lam reaction to make $\mathbf{1 5}$. Although this route did work, yields of the boronic acid, 20, were very inconsistent.

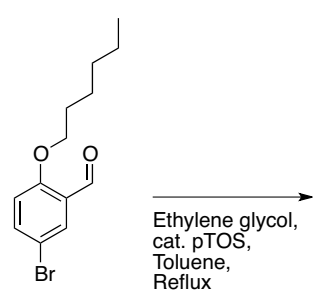

14

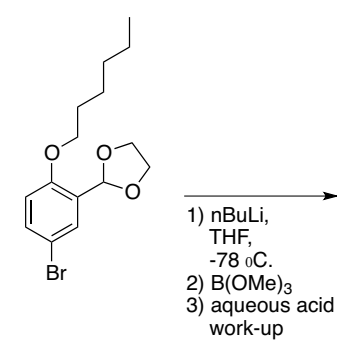

19

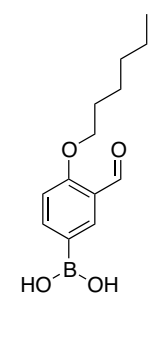

20

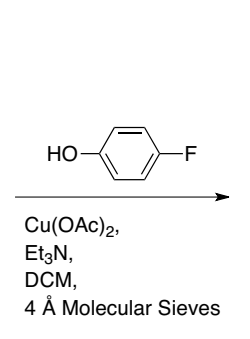

$4 \AA$ A Molecular Sieves

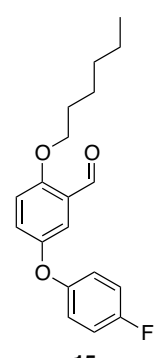

15

With poor results through the Chan-Lam route, it was decided to try the modified Ullmann ether synthesis ( $14 \rightarrow \mathbf{1 5}$ in FIGURE 2.2). ${ }^{69,70}$ Although the Ullmann ether synthesis is air sensitive like the Buchwald-Hartwig reaction, the reagents are less expensive and are easy to remove during the work-up. After an initial screen of ligands (2,2'-bipyridine, phenanthroline, N,N-dimethylglycine, N,N,N',N'-tetramethylethane-1,2-diamine, and tributylphosphine) and anhydrous solvents (DMF, DMSO, and dioxane), qualitatively TLC spot intensity indicated that phenanthroline in either DMF or DMSO worked best. It was also found that all combinations of solvent and ligands worked to some extent except for tributylphosphine and N,N-dimethylglycine in dioxane. Further screening found that a 1:1 ratio of phenanthroline to $\mathrm{CuI}$ produced the same result as the literature standard ratio of 2:1. Also, the use $\mathrm{K}_{3} \mathrm{PO}_{4}$ instead of $\mathrm{K}_{2} \mathrm{CO}_{3}$ decreased reaction yields. Among the conditions tested the highest yield occurred with two equivalents of $\mathrm{CuI}$ and phenanthroline and 4 equivalents of phenol slowly added to the reaction. The addition of KI to the reaction results in a modest improvement of the yield. In the final reaction toluene, about $10 \%$ total volume, was added to azeotrope off any 


\section{Cost-effective synthetic route to ET-92 and its analogs}

water present. That addition clearly does not reduce the reaction yield, but it is unclear whether or not it improves. As phenanthroline monohydrate was initially used successfully, this reaction is tolerant of some water. The major side-product of this reaction is 2-(hexyloxy)benzaldehyde, which co-elutes with $\mathbf{1 5}$ on silica and must be removed before continuing the synthesis. As $\mathbf{1 5}$ is a liquid, vacuum distillation would be a inexpensive purification method. Due to the high viscosity and expected boiling point of $\mathbf{1 5}$, multistage vacuum falling film distillation ${ }^{71}$ would be a good technique to use as it would reduce the hold-up. Since 15 was prepared on a small and micro-scale vacuum distillation equipment was not available, 15 was purified by $\mathrm{C}-18 \mathrm{rpHPLC}$.

While working on the reaction of 14 to $\mathbf{1 5}$, several experiments lead to notable observations. Acetonitrile does work as a reaction solvent, though poorly $(<25 \%$ yield), and yields large amounts of an orange powder that could be nano-copper particles, since the powder dissolved in aqueous $\mathrm{HCl}$ producing a blue-green solution that was visually similar to solutions of $\mathrm{CuCl}_{2}$. Also, $\mathrm{Cu}(\mathrm{OAc})_{2}$, instead of $\mathrm{CuI}$ works, but very poorly ( $<10 \%$ yield); however the addition of KI greatly improves the reaction yield. Conventional wisdom holds that the Ullmann reaction proceeds by oxidative addition of $\mathrm{Cu}^{+1}$ to the arylhalide resulting in a $\mathrm{Cu}^{+3}$ species or by transmetallization with $\mathrm{Cu}^{+2}$, however neither of these explain why $\mathrm{Cu}(\mathrm{OAc})_{2}$ works $\left(\mathrm{Cu}^{+4}\right.$ is unknown and there were no other metal species present 


\section{Cost-effective synthetic route to ET-92 and its analogs}

for $\mathrm{Cu}^{+2}$ to transmetalate with). One possible explanation is $\sigma$-bond metathesis, ${ }^{72}$

which was an unknown reaction when most of the mechanistic research was done

on the Ullmann reaction. The improved yield upon addition of $\mathrm{KI}$ to $\mathrm{Cu}(\mathrm{OAc})_{2}$

reaction mixture, is most probably due to a redox reaction:

$$
\begin{gathered}
\mathrm{Cu}^{+2}+\mathrm{e}^{-} \rightarrow \mathrm{Cu}^{+} \\
2 \mathrm{I}^{-} \rightarrow \mathrm{I}_{2}+2 \mathrm{e}^{-}
\end{gathered}
$$

The Grignard reaction, $\mathbf{1 5} \rightarrow \mathbf{1 6}$, FIGURE $\mathbf{2 . 2}$, is very clean and straight forward. A chiral variation has been reported by Fan $^{73}$ which if followed by a Mitsunobo with HCN could produce chiral ET-92 (FIGURE 2.5). However, these reactions were not pursued due to a desire to first develop an achiral ET-92 synthesis then use that route to develop new thyronamine analogs.

FIGURE 2.5: A possible route to chiral ET-92.

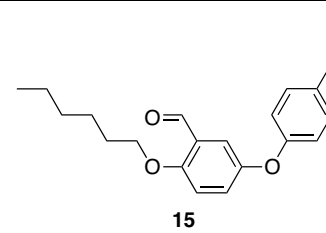

15
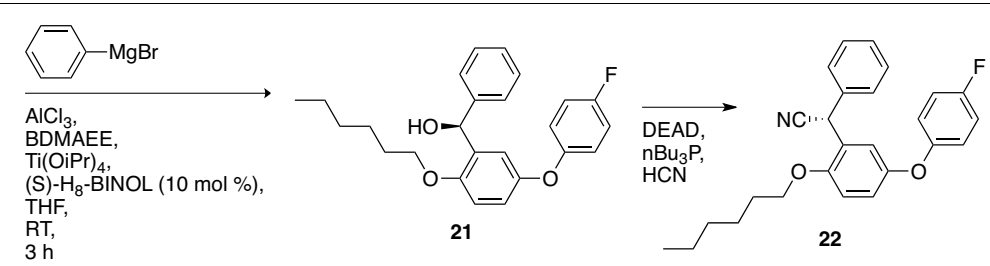

The nitrilation of 16, FIGURE 2.2, has been attempted with $\mathrm{pTosCl}$ and thionyl chloride followed by $\mathrm{KCN}$ before settling on the TMSCN/ $/ \mathrm{TBr}_{3}$ catalyzed reaction. ${ }^{74}$ When the test reaction $\mathbf{2 3} \rightarrow \mathbf{2 4}$ (FIGURE 2.6) was purified by basic alumina chromatography eluting with hexanes and EtOAc, which removed the sole major by-product, 4-bromo-2-((hexyloxy)-(phenyl)methyl)phenol, ${ }^{1} \mathrm{H}-\mathrm{NMR}$ of 24 in DMSO- $\mathrm{d}_{6}$ revealed that the proton next to the nitrile has a chemical shift of 5.78 


\section{Cost-effective synthetic route to ET-92 and its analogs}

ppm which previously had been mistaken as residual DCM (5.76 ppm) due the use

of house vacuum and the inability to completely remove solvents from samples.

Armed with that information, samples of $\mathbf{1 7}$ made with pTosCl and thionyl chlo-

ride were repurified on basic alumina, confirming that those reactions had also

worked but were not as pure nor as high yielding as the $\mathrm{TMSCN} / \mathrm{InBr}_{3}$ catalyzed

reaction. Another advantage of the $\mathrm{TMSCN} / \mathrm{InBr}_{3}$ reaction is that it is so fast that it

might be complete within seconds of mixing the reagents, thus allowing for the

reaction to be done in a continuous flow reactor with an inline basic alumina plug

filtration, and significant cost savings.

FIGURE 2.6: Nitrilation reaction confirmed the feasibility of the new ET-92 route. This reaction is complete in less than 30 minutes, possibly seconds, with only one major side product: 4-bromo-2-((hexyloxy)(phenyl)methyl)phenol at 16\%.

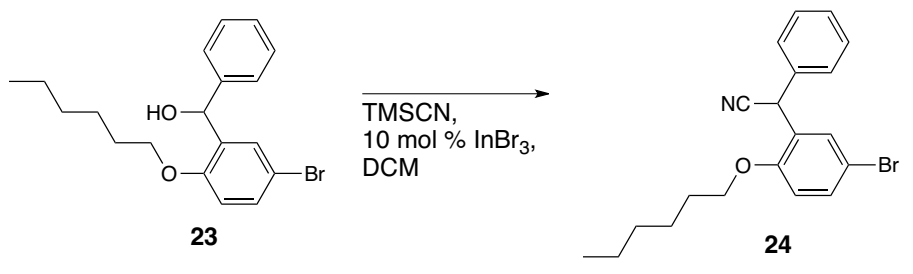

The reduction of $\mathbf{1 7}$ to ET-92 proved to be problematic, with the highest yield to date being $53.9 \%$ using $\mathrm{BH}_{3} \cdot \mathrm{DMS}$ in refluxing THF overnight. ${ }^{75}$ When the hard reducing agents $\left(\mathrm{LiAlH}_{4}\right.$ and Dibal-H) were used, the yields are very low $(\sim 10 \%)$ with large amounts of side-products. Reduction with $\mathrm{Pd} / \mathrm{C}$ had no effect.

In summery, a novel synthetic route was developed for ET-92, the lead compound for this project, which reduced the number of synthetic steps from 14 to 5 


\section{Cost-effective synthetic route to ET-92 and its analogs}

and improved the overall yield from $15.3 \%$ to $18.3 \%$ (77.4 mg). Although this was not a production this sets the table for future scale-up work. 
With the working hypothesis that TAAR1 activation by Meth is significantly responsible for desirable characteristics of Meth intoxication, the development of TAAR1 inverse agonists (basal inhibitors) as treatments for Meth addiction may not be possible, since they could push the Meth user further away from how they want to feel. However, the development of selective TAAR1 antagonists are needed to pharmacologically determine the role of TAAR1-mediated signaling in vitro and in vivo. In future work, the development of partial or fully agonists could provide effective treatments.

\subsection{Initial attempts to synthesize new analogs}

Once the new ET-92 synthetic route had been developed, ET-92 analogs were postulated that had greater water solubility and further explored the structureactivity-relationships (SAR) of the thyronamine family. Thus, the synthesis of compounds 1-2 (FIGURE 3.1) was begun.

FIGURE 3.1: First new analog targets to be synthesized.
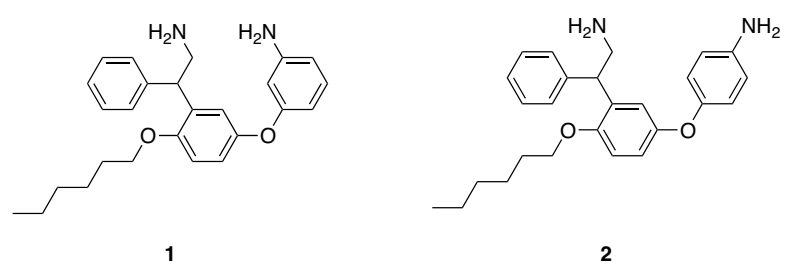


\section{Change in strategy and targets}

To make compounds $\mathbf{1}$ and $\mathbf{2}$, the Ullmann reaction between $\mathbf{3}$ and either metaor para-nitrophenol was attempted, FIGURE 3.2. Unfortunately, the reactions did not produce a detected amounts of product, probably due to increased acidity of para- and meta-nitrophenol (pka 7.12 and 8.36 respectfully) compared to 4-fluorophenol (pka 9.81) and phenol (pka 10.02). Ullmann reaction works best with amines, then amides and finally alcohols but not at all with carboxylic acids. ${ }^{69}$ In other words, reaction yields decrease as acidity of the nucleophile increases. After several attempts with no evidence of the desired products, compounds $\mathbf{1}$ and $\mathbf{2}$ were triaged to low priority.

FIGURE 3.2: The first step in synthesizing compounds 1 and $\mathbf{2}$.

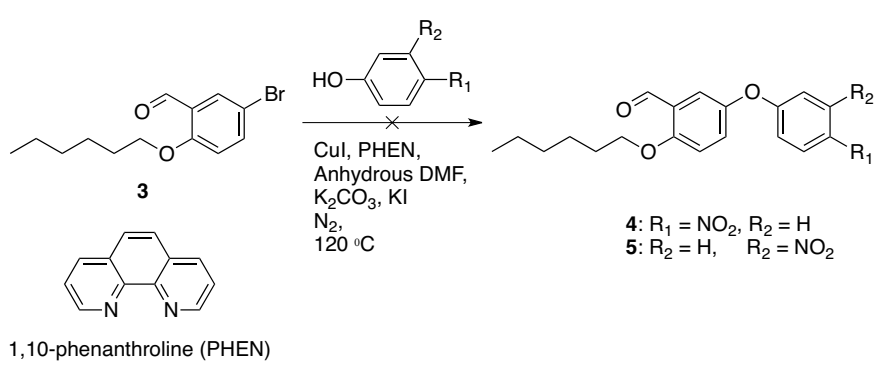

\subsection{Change in strategy and targets}

Due to the synthetic difficulties outlined above, and the desire to have target compounds with improved water solubility, syntheses of the molecules depicted in FIGURE 3.3 were pursued next. Although the 4-aminophenol ${ }^{76}$ core of $\mathbf{6}$ - 14 may have similar toxicity issues to the hydroquinone ${ }^{77}$ core of 1, 2, and ET-92, it allows one to develop different chemistries to explore the SAR. 


\section{Change in strategy and targets}

FIGURE 3.3: New targets and ET-92

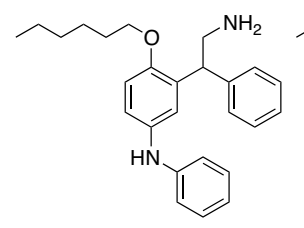

6

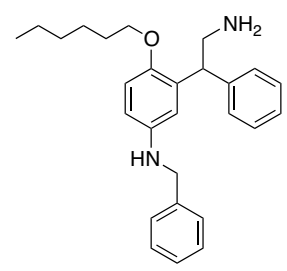

10

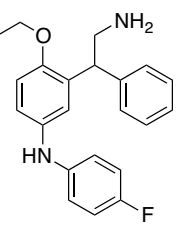

7

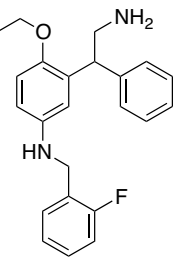

11

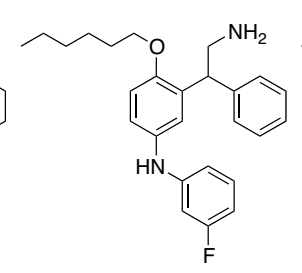

8

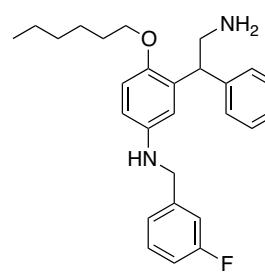

12

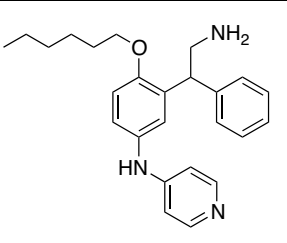

9

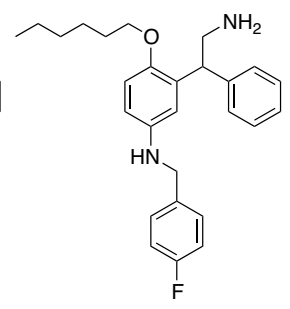

13

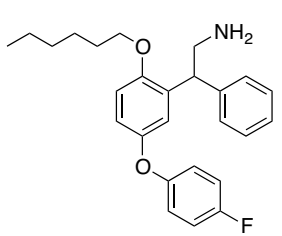

ET-92

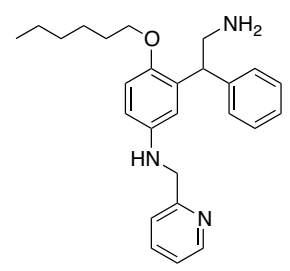

14

Starting with 5-nitrosalicylic acid, $\mathbf{1 5}$, the synthesis proceeded smoothly through three steps, FIGURE 3.4. An attempt to couple 4-pyridylboronic acid and 17 under Chan-Lam conditions did not work, but that was not especially surprising as pyridylboronic acids are known to be difficult coupling partners. Yields on the Chan-Lam reactions, 19 a-c, were $\sim 35-42 \%$ while the reductive aminations, 18 a-d and 32, were $\sim 53-61 \%$. In going from 16 to 17 , acetic acid was added to the reaction mixture to promote hydrogen transfer in the aprotic ethyl acetate and to prevent the amine from chelating the palladium, which can be an issue. Later work demonstrated that addition of acid was not needed during the hydrogenation. 


\section{Change in strategy and targets}

FIGURE 3.4: The first steps towards the new analogs.

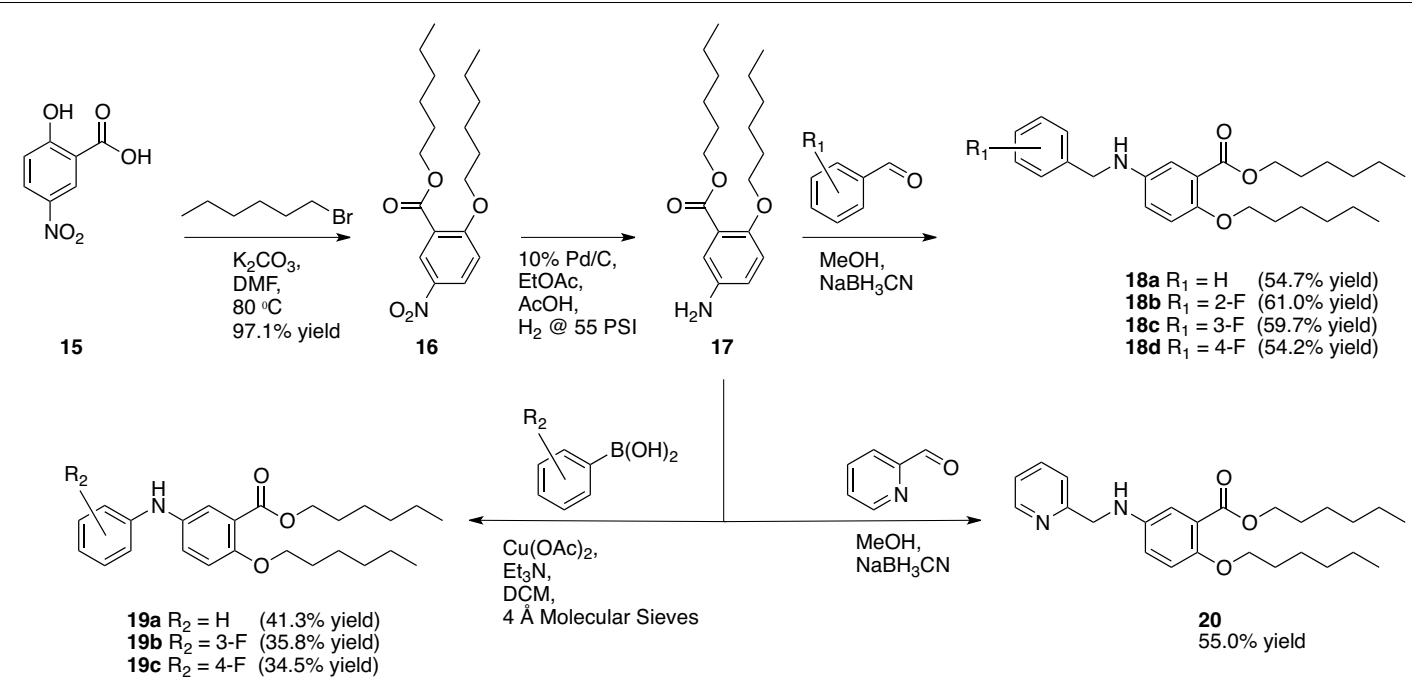

Reduction of the esters to aldehydes followed, and, although showing promise, only resulted in $\sim 10 \%$ yields by ${ }^{1} \mathrm{H}-\mathrm{NMR}$, FIGURE 3.5. The $\mathrm{Et}_{2} \mathrm{NH}, \mathrm{LiAlH}_{4}$ and hexanes were premixed, then the ester was added. ${ }^{78}$ Since no benzyl alcohols nor carboxylic acids were detected by ${ }^{1} \mathrm{H}-\mathrm{NMR}$, there was simply not enough reducing agent present to drive the reaction to completion. The aniline amine may have also reacted with some of the reducing agent, limiting the effectiveness of the reaction. With more development this reaction could prove useful, however further development was set aside.

FIGURE 3.5: Reduction of ester to aldehyde.

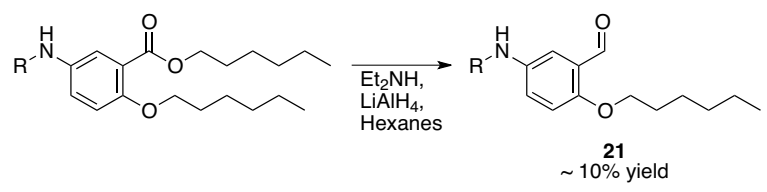


Another strategy change

\subsection{Another strategy change}

Since the reduction of the esters did not immediately result in high yields of the corresponding aldehydes, the synthetic route was once again changed. The plan became to form a tertiary amide then convert the amide to a ketone by reacting it with a Grignard reagent.

Starting with 5-nitrosalicylic acid and pyrrolidine, the amide (22) was formed with the use of either EDCI, DCC, or DCC and $\mathrm{HOBT} \cdot \mathrm{H}_{2} \mathrm{O}$, FIGURE 3.6. In all cases, the yields were highly variable ( $20-91 \%$ by mass) with no obvious reason for the differences. A survey of SciFinder results for amide formation with various salicylic acids revealed a similar range of yields. Although the use of HOBT produced the highest yield, the product contained impurities that were difficult to remove. The rest of the sequence to $\mathbf{2 4}$ is straightforward.

FIGURE 3.6: Synthesis of a key intermediate.

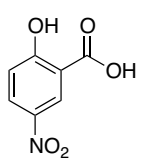

15
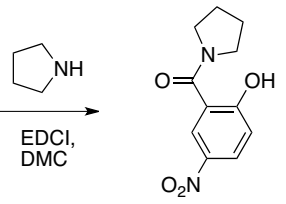

22

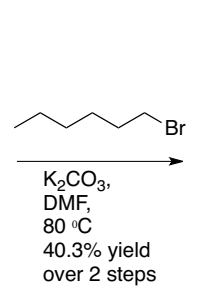

over 2 steps

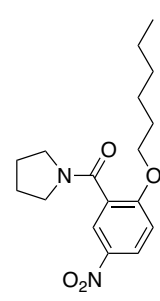

23

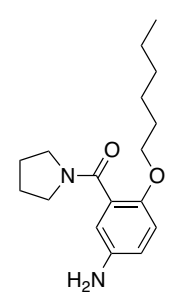

24

As before, the amine of $\mathbf{2 4}$ was functionalized through either reductive amination or the Chan-Lam reaction, FIGURE 3.7. When the Grignard reaction of 25a, FIGURE 3.8, was attempted, nothing happened despite using 5 equivalents of phenylmagnesiumbromide. The large amount of Grignard reagent was sufficient to 


\section{Another strategy change}

react with the residual water in the THF, deprotonate the amine, and yet leave enough to react with the amide. The amide's lack of reactivity maybe the result of steric hindrance, as ${ }^{1} \mathrm{H}-\mathrm{NMR}$ reveals that the pyrroldinamide exists as a rotamer with the pyrrolidine ring askew, but not perpendicular, to the central phenyl as evidenced by the $\sim 0.32 \mathrm{ppm}{ }^{1} \mathrm{H}-\mathrm{NMR}$ shift difference between the methylene protons on either side of the pyrrolidine's nitrogen. However, one might think that with the amide being co-planar with the adjacent phenyl ring that the Grignard reagent would have a clear path for a facial attack on the amide.

FIGURE 3.7: Functionalizing the amine.

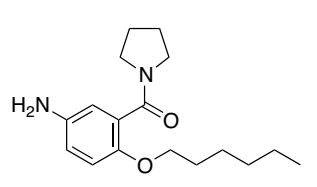

24
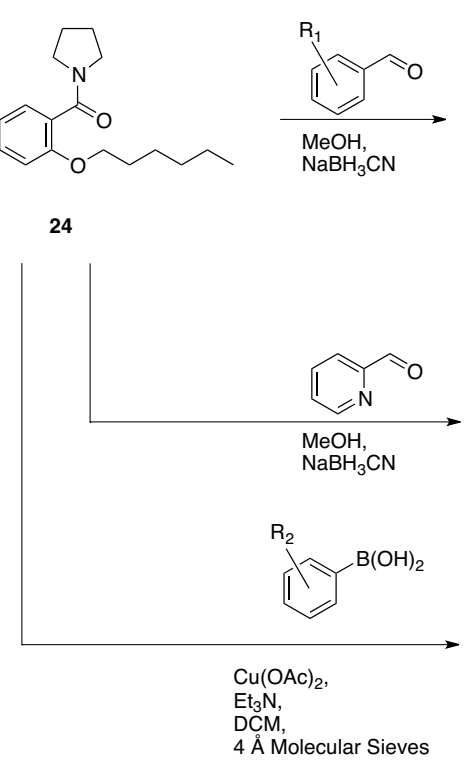<smiles>CCC1=CC=[Pb]C1</smiles><smiles>COc1ccc(NN)cc1C(=O)N1CCCC1</smiles>

25a: G5-103s10 $R_{1}=H$ (5) $(\sim 95 \%$ yield $)$ 25d: G5-110s8 $\quad R_{1}=2-F$

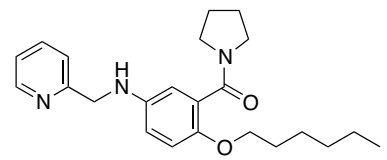

25e: G5-111s8

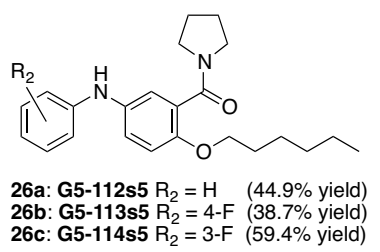

FIGURE 3.8: A failed Grignard reaction.

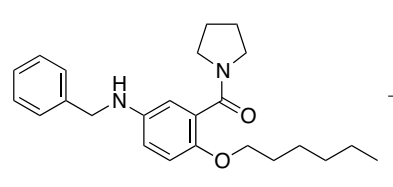

$25 a$

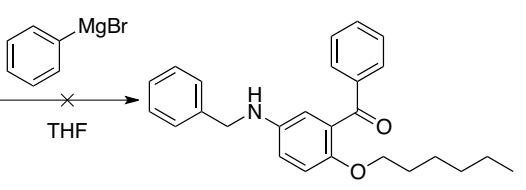

ก) 
Final attempt to synthesize 6 - 14

\section{$3.4 \quad$ Final attempt to synthesize $6-14$}

FIGURE 3.9: The final synthesizes of thyronamine analogs.

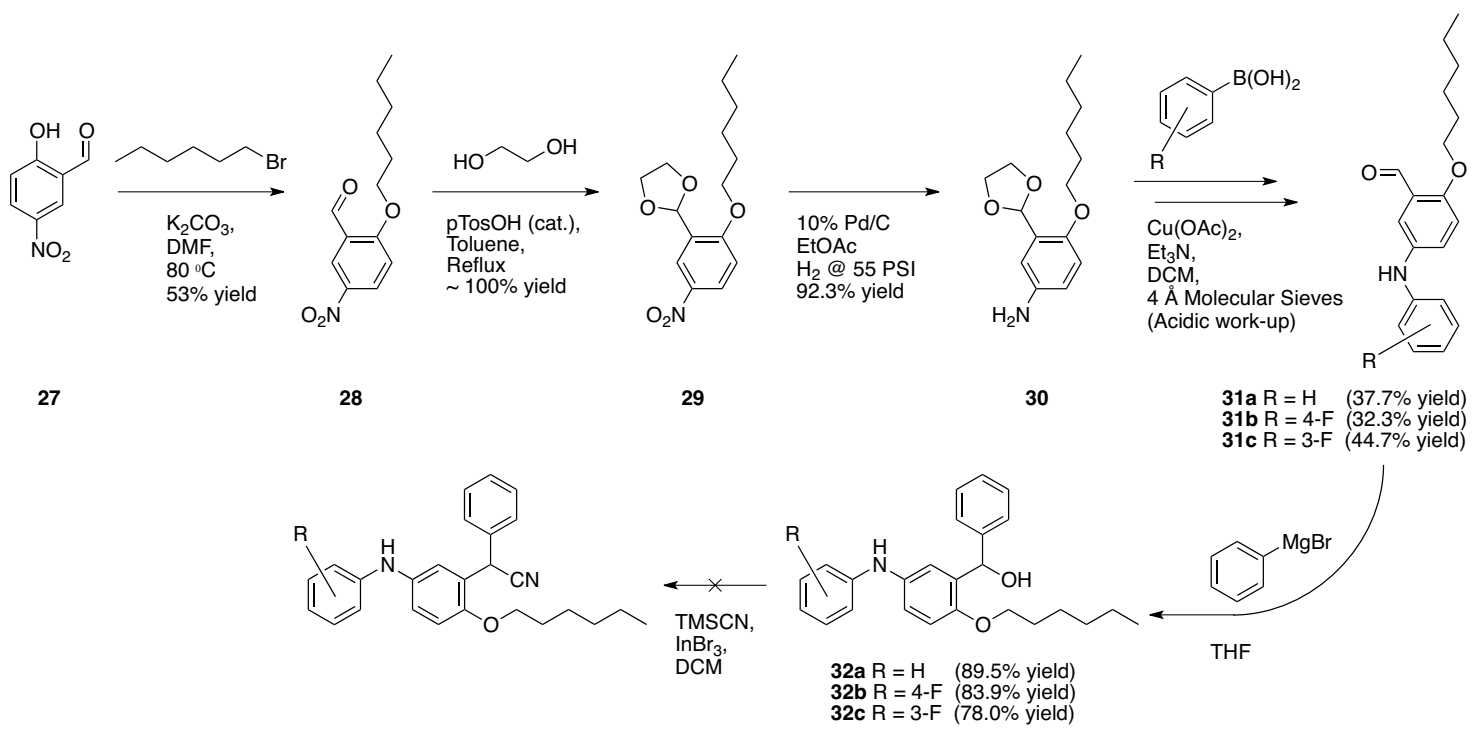

Upon acquiring 5-nitrosalicylaldehyde, 15, the final synthesis, shown in FIG-

URE 3.9, was started. The reaction sequence worked well except for the last step.

The nitrilation reaction might not have worked well due to the amine in 32a-c forming a salt with cyanide or the amine forming a complex with indium. There

was ${ }^{1} \mathrm{H}-\mathrm{NMR}$ evidence that some TMS ether of 32a-c formed, indicating that extraneous water was probably not the cause of the poor yield. In the future, one might attempt to form the nitrile by reacting 32a-c with either p-toslyl chloride or thionyl chloride followed by displacement with $\mathrm{KCN}$. An attempt to methylate the amine had been made after the Chan-Lam reaction, 31, but was unsuccessful. Not shown in FIGURE 3.9, was the unsuccessful attempt to reductively aminate $\mathbf{3 0}$ with various aldehydes, which was not considered significant since the equivalent products could be made in the future with the appropriate benzyl halides. 
Final attempt to synthesize 6 - 14

At this point, it was decided to assay G5-109s8 through G5-114s5 in oocytes injected with hTAAR1 and hCFTR encoding RNA. Although these compounds were synthesized as intermediates to $6,7, \mathbf{8}, 12,13$, and 14 , they comprise interesting SAR in their own right. (S)-sulpiride (FIGURE 3.10, a $\mathrm{D}_{2}$ and $\mathrm{D}_{3}$ receptor antagonist approved in several countries outside the USA for use as an antipsychotic) was considered a template molecule during the original TAAR1 ligand development project, ${ }^{17}$ however the only compounds synthesized in that project that contained an amide group were analogs of ET-10, so G5-109s8 through G5114s5 explore a gap in the TAAR1 SAR. Also, G5-109s8 through G5-114s5 contain similar peripheral SAR as ET-92: the consistent 2-hexyl ether; and the variable SAR aromatic group at the 5 position, as well as a rigid cyclic functional group one carbon off the 1 position of the core phenyl ring. 


\section{Final attempt to synthesize $6-14$}

FIGURE 3.10: $\mathrm{SAR}$ comparison between (S)-sulpiride (a $\mathrm{D}_{2}$ and $\mathrm{D}_{3}$ receptor antagonist), ET-10 (a TAAR1 agonist), ET-92 and G5-109s8 through G5-114s5. Although (S)-sulpiride was considered a template molecule during the original TAAR1 ligand development project, ${ }^{17}$ analogs of ET-10 were the only one made that contained amides. G5-109s8 has the same 1, 2, 5 substitution pattern as (S)-sulpiride and ET-92. It also similar peripheral SAR as ET-92: the hexyl ether; fluorinated aromatic; and rigid third cyclic functional group, pyrrolidine instead of the phenyl ring of ET-92. Thus, even though G5-109s8 was synthesized as an intermediate to $\mathbf{2 4}$, it has SAR worth testing in its own right.

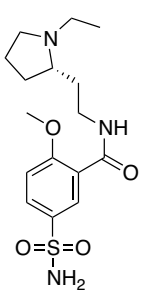

(S)-sulpiride

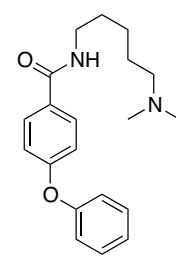

ET-10

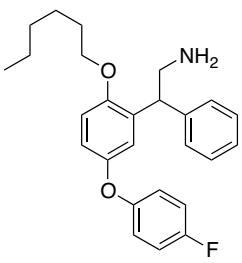

ET-92

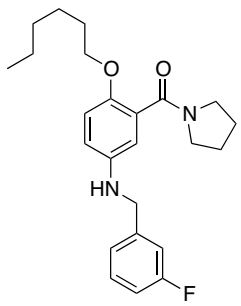

G5-109s8

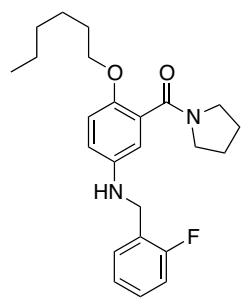

G5-110s8

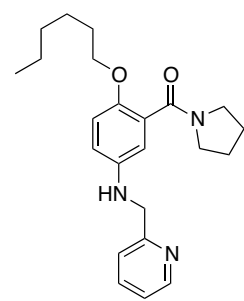

G5-111s8

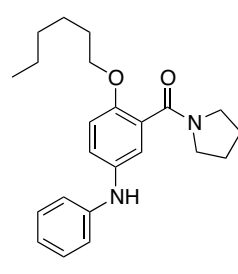

G5-112s5

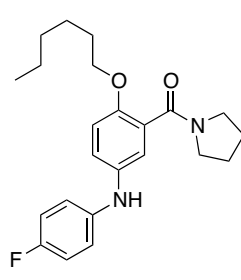

G5-113s5

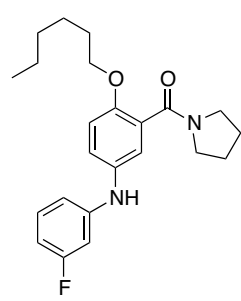

G5-114s5 
The compounds shown in FIGURE 4.1 were evaluated for TAAR1 activity by monitoring the transmembrane conductance of Xenopus Laevis (frog) oocytes injected with hTAAR 1 and hCFTR ${ }^{35}$ encoding RNA or just hCFTR encoding RNA as the control via two electrode voltage clamp (TEVC). The oocytes were harvested and prepared by Dr. Yohei Norimatsu according to the procedure in Chapter 7.2. As stated in Chapter 1.1, a downstream effect of TAAR1 activation is increased production of cAMP, where as CFTR is activated by cAMP and upon activation increases the transmembrane conductance by allowing chloride ions to cross the cell's plasma membrane. Thus an oocyte expressing both TAAR1 and CFTR acts as a biological circuit for measuring the activity of TAAR1.

FIGURE 4.1: Compounds tested
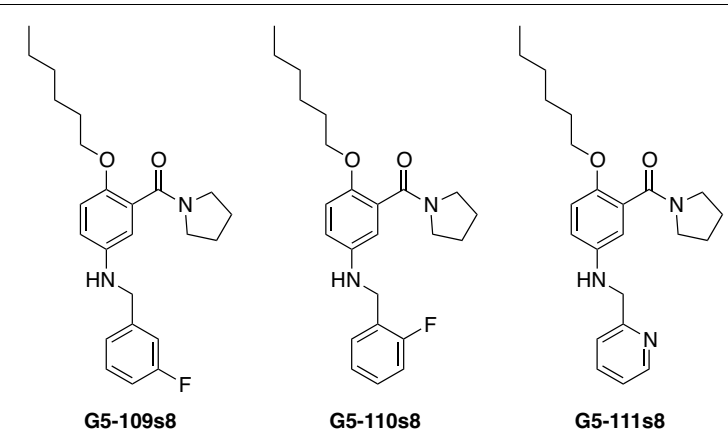

G5-111s8
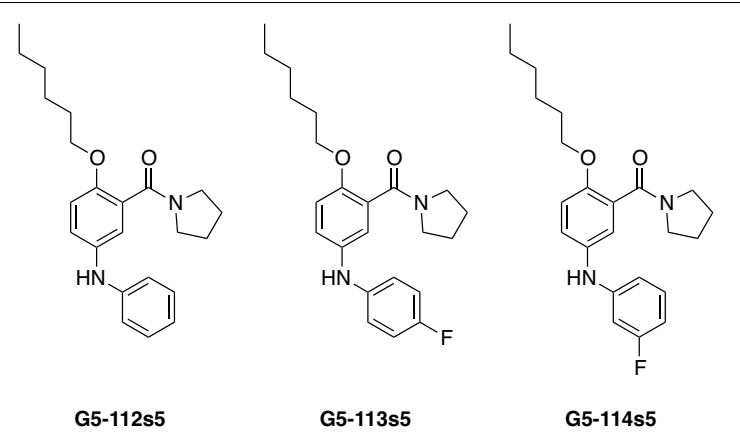

These compounds could be evaluated by fluorescent cellular assays sensitive to cAMP levels using HEK cells transfected with mouse TAAR1 (mTAAR1). The 
The experiments

quality of results from this type of cellular assay depend on the accuracy and consistency of one's pipetting, and the stability of the cell line. Other draw backs of this technique are that it only provides one type of data (cAMP concentrations) and the fact that the project is to treat human Meth addiction not mouse Meth addiction. The fluorescent assays have the advantage of being well suited for highthrough-put screening, especially if one has access to robotic pipetting systems.

Although highly automated TEVC systems exist, including machines that inject oocytes, the system used for this work was manual with computerized data collection, please see Chapter 7.2.3 for more details. Also, the oocytes were manually injected, Chapter 7.2.2. The advantages of TEVC are that the oocytes are prepared weekly, eliminating potential cell-line stability problems though expression levels can still be an issue; both transmembrane conductance and potential can be monitored independent of what causes the changes. Finally, TEVC allows one to conduct many different variations of steady-state or time-dependent response experiments. Also, by monitoring transmembrane conductance and potential, TEVC allows one to detect off-target effects mechanistically unrelated to cAMP levels, a significant advantage over cAMP fluorescent assays, which only assay cAMP concentrations.

\subsection{The experiments}

All assays were preformed once as first pass activity screens. 
The experiments

For all oocytes injected with both hTAAR1 and hCFTR encoding RNA, 10

times more hTAAR1 cRNA, by mass, was injected into the oocytes than hCFTR

cRNA. This was a deliberate attempt to observe basal activity of TAAR1, as the

unperturbed transmembrane conductance of the oocyte would increase as the

expression of TAAR1 increased if TAAR1 had basal activity.

FIGURE 4.2: (A) Dose (concentration * 1.5 minutes $* 4 \pm 1 \mathrm{~mL} / \mathrm{minute}$ ) response testing of Meth in oocytes injected with hTAAR and hCFTR cRNA. The dose, indicated by the labeled red regions, was perfused for 1.5 minutes followed by perfusing with Frog Ringer's solution until the next dose $(n=1)$. The red line is an approximate baseline. (B) Dose response curve for (A). The experiment was performed by Dr. Yohei Norimatsu, who provided the data to the author.
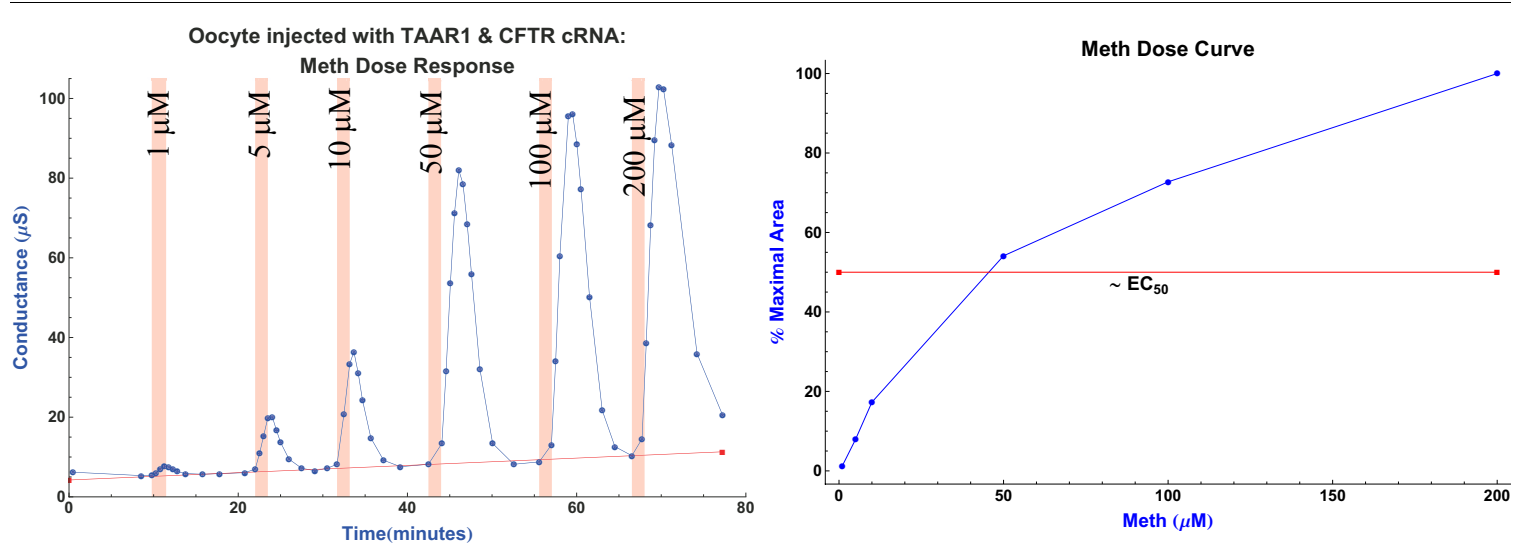

The Meth dose (concentration * 1.5 minutes $* 4 \pm 1 \mathrm{~mL} /$ minute) response experiment in FIGURE 4.2 was performed by Dr. Yohei Norimatsu, using an oocyte injected with hTAAR1 and hCFTR encoding RNA, and using $1 \mu \mathrm{M}, 5 \mu \mathrm{M}$, $10 \mu \mathrm{M}, 50 \mu \mathrm{M}, 100 \mu \mathrm{M}$ and $200 \mu \mathrm{M}$ Meth in FR pulses (red shaded regions) followed by washing with FR. Integration of the area under the curves show that the $10 \mu \mathrm{M}$ Meth peak has an area of $\sim 18 \%$ of the $200 \mu \mathrm{M}$ Meth peak and the $100 \mu \mathrm{M}$ Meth peak is about equal to the $200 \mu \mathrm{M}$ Meth peak. The $\mathrm{EC}_{50}$ for Meth in this experiment is $\sim 50 \mu \mathrm{M}$ Meth. The choice to go with Dr. Norimatsu's recommenda- 


\section{The experiments}

tion of using $10 \mu \mathrm{M}$ Meth for the compound assays was made prior to analyzing this data.

A $100 \mathrm{mM}$ stock solution of each test compound, in DMSO, was made, and then used to make the test solutions.

With the exception of the first and last compound assay (FIGURE 4.4 and 4.6(1), respectfully), all assays followed a head-to-head competition assay paradigm where a 1.5 minute pulse of $10 \mu \mathrm{M}$ Meth in Frog Ringer's solution (FR) was perfused over an oocyte, injected with hTAAR1 and hCFTR encoding RNA, followed by a wash-out period with FR. After the initial Meth pulse and wash-out, a series of 1.5 minute pulse of $10 \mu \mathrm{M}$ Meth and test compound ( $1 \mathrm{nM}$ to $10 \mu \mathrm{M})$ in FR was followed by FR wash-out cycles were preformed. At the end of each experiment, a 1.5 minute pulse of $10 \mu \mathrm{M}$ forskolin ${ }^{79}$ and $10 \mu \mathrm{M}$ of the test compound in FR followed by a FR wash-out period. The wash-out periods lasted between 15 and 25 minutes (long enough for the conductance to stabilize). The application of forskolin and test compound at the end of the experiments served as a control to demonstrate that the test compound was not blocking either CFTR or adenylyl cyclase. Although some may criticize these experiments because they did not follow the common practice of pretreating with the test compound followed by agonist, the shape of the response curves provide kinetic information. These head- 
The experiments

to-head competition experiments were designed by analogy to stopped-flow chem-

ical kinetics experiments. ${ }^{80}$

FIGURE 4.3: Structure of Forskolin.

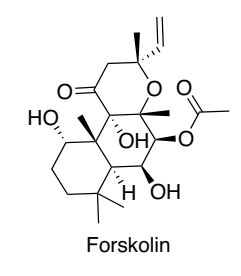

In the first assay, FIGURE 4.4, an oocyte injected with hTAAR1 and hCFTR encoding RNA was used. The Meth pulses were 1.5 minutes long and at $100 \mu \mathrm{M}$ concentration in FR. Each Meth pulse was followed by a 15 minute FR wash-out period. After the second pulse of Meth, $20 \mu \mathrm{M}$ G5-109s8 in FR was applied immediately followed by a Meth pulse. No forskolin control was done in this experiment.

For the last assay, FIGURE 4.6(1), an oocyte injected with hCFTR encoding RNA was used. Between each pulse of testing solution, the oocyte was washed with FR. The 1.5 minute pulses used were: $5 \mu \mathrm{M}$ forskolin, $10 \mu \mathrm{M}$ G5-109s8, 10 $\mu \mathrm{M}$ Meth, and a combination of $5 \mu \mathrm{M}$ forskolin and $10 \mu \mathrm{M}$ G5-109s8. All of these solutions were made using FR.

The TEVC used in these experiments required that the siphon from the solution reservoirs to the recording chamber to be manually pinched closed before it was transferred between reservoirs. The manual transfer had the potential to create 
Results

air bubbles in the siphon (uncertainty in the volume of solution applied) and timing

uncertainty leading to errors estimated at $\pm 10 \%$. Both of those issues could be

reduced or eliminated through the use of an automated multi-valved perfusion sys-

tem, such as the Octaflow IITM from ALA Scientific Instruments. ${ }^{81}$ Another source

of error in the analysis of these experiments was the need to estimate the observed

peaks' baselines before integrating their areas, which is a subjective process. The

final concern with these assays was the variability of expression levels of hTAAR1

and hCFTR in the oocytes. The variability could be due to variance in cRNA injec-

tion volumes. Since these experiments were intended to be initial compound activity screens and concerns of measurement uncertainties, the results will be

discussed qualitatively.

\subsection{Results}

\subsubsection{Testing G5-109s8}

Of the compounds to be tested, G5-109s8 was the purest by ${ }^{1} \mathrm{H}-\mathrm{NMR}$, which is why it was the most heavily tested in the limited time available. Also, being an oil, it dissolved into DMSO faster than the other test compounds, and thus it was the first tested.

In the first experiment, FIGURE 4.4, $20 \mu \mathrm{M}$ G5-109s8 (green shaded region) caused the rapid drop in transmembrane conductance. The pulse of Meth (peak C) immediately following the pulse of G5-109s8 is visibly smaller than all other 
Results

peaks, including peak A, which was the result of a fumbled siphon transfer resulting in less than the full 1.5 minute pulse of Meth. Although peak F is smaller then E-G, the difference is not large enough for a single trial to be confident that it is not the result of some error. The small size of peak C is strong evidence that G5-109s8 is blocking the TAAR1 pathway, if not TAAR1 itself. This experiment was originally envisioned as part of a series of experiments to determine the $\mathrm{K}_{\mathrm{d}}$ and $\mathrm{IC}_{50}$ of each test compound, however upon realizing how time consuming that was going to be a head-to-head competition assay was designed for the rest of the testing.

FIGURE 4.4: Pretreatment with $20 \mu \mathrm{M}$ G5-109s8 (magenta region) for 1.5 minutes followed by 1.5 minute pulses of $100 \mu \mathrm{M}$ Meth (red regions). Although the original intent was to measure the off-rate of G5-109s8, peak $C$ is visibly smaller than all others indicating that G5-109s8 inhibited Meth binding to TAAR1. The gray line segments are the approximate baseline. It is worth noting that G5-109s8 caused a drop in the baseline. Peak A represents a fumbled siphon transfer, so a second Meth control stimulation, B, was preformed. This experiment was preformed once.

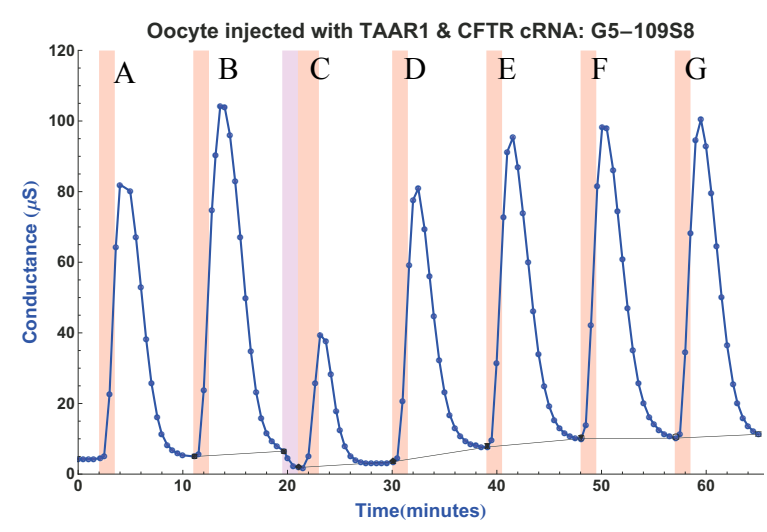

In FIGURE 4.5, one sees the dose response curves for the head-to-head competition assay between G5-109s8 and $10 \mu \mathrm{M}$ Meth (each pulse, B-F and I-L, was a mixture of both compounds). In FIGURE 4.5 the doses of G5-109s8 were B: $1 \mathrm{nM}$, C: 10 nM, D: 100 nM, E: $1 \mu \mathrm{M}, \mathrm{F}: 10 \mu \mathrm{M}, \mathrm{I}: 1 \mu \mathrm{M}, \mathrm{J}: 3 \mu \mathrm{M}, \mathrm{K}: 5 \mu \mathrm{M}$ and L: $7 \mu \mathrm{M}$. The weak response to Meth, peak H, in the experiment shown in FIGURE 4.5(2) 


\section{Results}

indicates that the oocyte was not expressing a lot of TAAR1 but there was enough to establish that G5-109s8 inhibits Meth stimulation of the TAAR1 pathway in a dose dependent manner. Since G5-109s8 caused a maximal depression of transmembrane conductance $\sim 4.5$ minutes after the end of pulse F, and the transmembrane conductance took $\sim 17$ minutes to return to basal levels after pulse $\mathrm{F}$ but only $\sim 10$ minutes after pulse A (just Meth), then G5-109s8 must have a slower off-rate than Meth.

FIGURE 4.5: Heads up competition between Meth and G5-109s8 ( $\mathrm{n}=1$ for both assays). Pulses were 1.5 minutes in duration. Grey line segments are the approximate baseline. (1) A) $10 \mu \mathrm{M}$ Meth, B) $10 \mu \mathrm{M}$ Meth \& 1 nM G5-109s8, C) $10 \mu$ M Meth \& 10 nM G5-109s8, D) $10 \mu$ Meth \& 100 nM G5-109s8, E) $10 \mu \mathrm{M}$ Meth \& $1 \mu$ M G5-109s8, F) $10 \mu$ M Meth \& $10 \mu$ M G5-109s8, G) 10 mM G5-109s8 \& 10 mM forskolin. (2) H) 10 mM Meth, I) 10 mM Meth \& 1 mM G5-109s8, J) 10 mM Meth \& 3 mM G5-109s8, K) 10 mM Meth \& 5 mM G5-109s8, L) 10 mM Meth \& 7 mM G5-109s8.
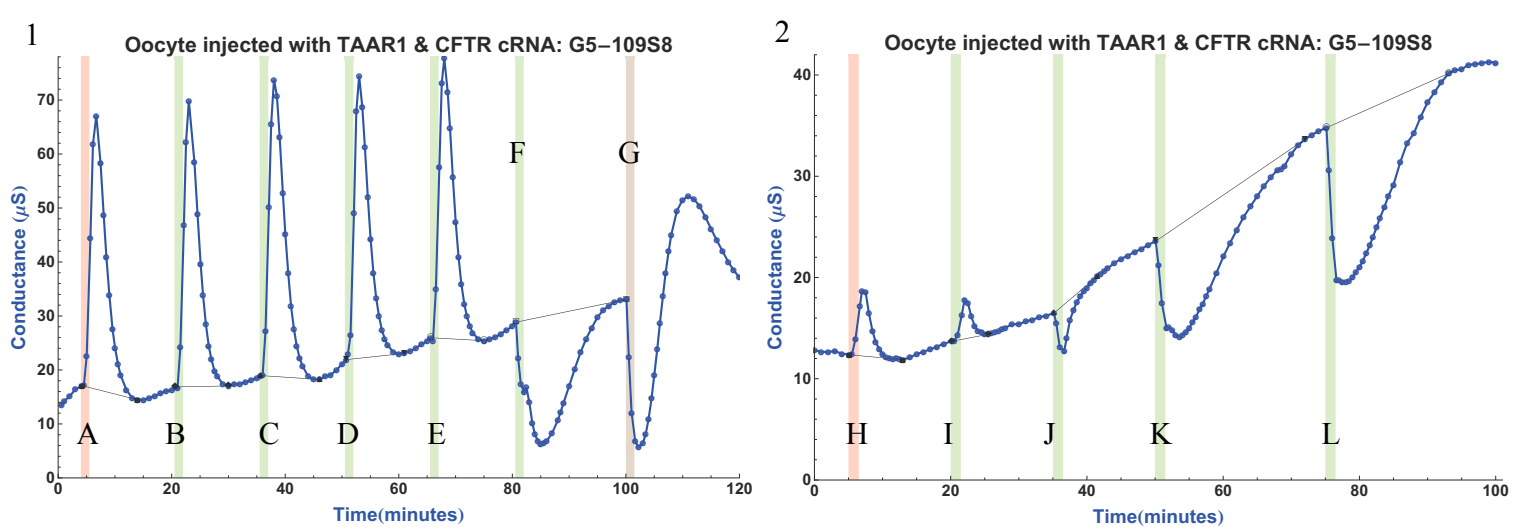

For G5-109s8 to cause sub-basal transmembrane conductance as in FIGURE

4.5(G, J, K, and L) the TAAR1 pathway must have basal activity, and G5-109s8 must be bound to nearly all free binding sites of whatever it is binding to in the pathway. If G5-109s8 only prevented Meth from reaching TAAR1 (by inhibiting DAT, for example), then G5-109s8 would have no effect on the TAAR1 pathway's 


\section{Results}

basal activity. Therefore G5-109s8 must be binding to either TAAR1, $G_{\alpha s}, G_{\beta / \gamma}$, adenylyl cyclase or CFTR.

From the response to forskolin and G5-109s8 (10 $\mu \mathrm{M}$ each) in FIGURE 4.5G, it can be concluded that neither adenylate cyclase nor CFTR were blocked by G5109s8. Although there is initially a large drop in conductance for pulse G, the trough is considerably narrower than the one in $\mathrm{F}$ and appears to be the independent sum of fast TAAR1 inhibition by G5-109s8 followed by a slower but strong activation of adenylyl cyclase by forskolin. This conclusion is consistent with Occam's razor (explanations with the fewest assumptions are preferred), since G5109s8 is an analog of a family known to inhibit TAAR1. If G5-109s8 was inhibiting adenylyl cyclase or even one of the G-protein subunits instead of TAAR1, then G5-109s8 would have unacceptable off-target effects as a treatment for addiction both adenylyl cyclase and the G-proteins are involved in too many other regulatory pathways to be safely inhibited for this application. However, inhibiting adenylyl cyclase or the G-proteins might be acceptable for something like chemotherapy. Similarly, an argument that G5-109s8 is inhibiting CFTR is an argument for an unacceptable off-target effect (in this case, one that would resemble cystic fibrosis). Before one spends more time developing analogs like G5-109s8, experiments that definitely rule out the inhibition of adenylyl cyclase, the G-proteins and CFTR by G5-109s8 should be done. One possible experiment could be a TEVC experiment with a hTAAR1 and hCFTR expressing oocyte where one perfused $10 \mu \mathrm{M}$ 
Results

G5-109s8 until the transmembrane conductance was constant, then perfused with a

1.5 minute pulse of $10 \mu \mathrm{M}$ G5-109s8 and $10 \mu \mathrm{M}$ forskolin solution followed by

washing with $10 \mu \mathrm{M}$ G5-109s8. If that experiment had a forskolin response equal

to a TAAR1 and CFTR expressing oocyte assayed with a 1.5 minute pulse of 10

$\mu \mathrm{M}$ forskolin, then neither adenylyl cyclase nor CFTR can be inhibited.

In FIGURE 4.5, one begins to see a general trend followed by the rest of the assays, namely the increase in transmembrane conductance as the experiments proceed. Not shown, but observed, was the time-dependent, and possibly dosedependent, drop of the transmembrane potential, typically about $50 \%$ by the end of the experiment. Currently, it is not clear how much of the long-term changes in the conductance and potential are the result of the oocytes response to stress or some auxiliary effect of the compounds being tested.

FIGURE 4.6: (1) CFTR only control experiment to detect off-target effects. Pulses were 1.5 minutes in duration. A) $5 \mu \mathrm{M}$ forskolin, B) $10 \mu \mathrm{M}$ G5-109s8, C) $10 \mu \mathrm{M}$ Meth, D) $10 \mu \mathrm{M}$ G5-109s8 \& $5 \mu \mathrm{M}$ forskolin. Grey lines are visual aids. (2) CFTR only control experiment to detect off-target effects. This experiment was a prior result from the Grandy lab $(n=1)$. Unfortunately, the notes about it how it was performed were a bit ambiguous, but it appears from them that the compounds were perfused over the oocyte for the entire period of the compound's shaded region. However, the response to ET-92 looks like the response one would expect from a minute or so application. The concentration of the forskolin used was not recorded.
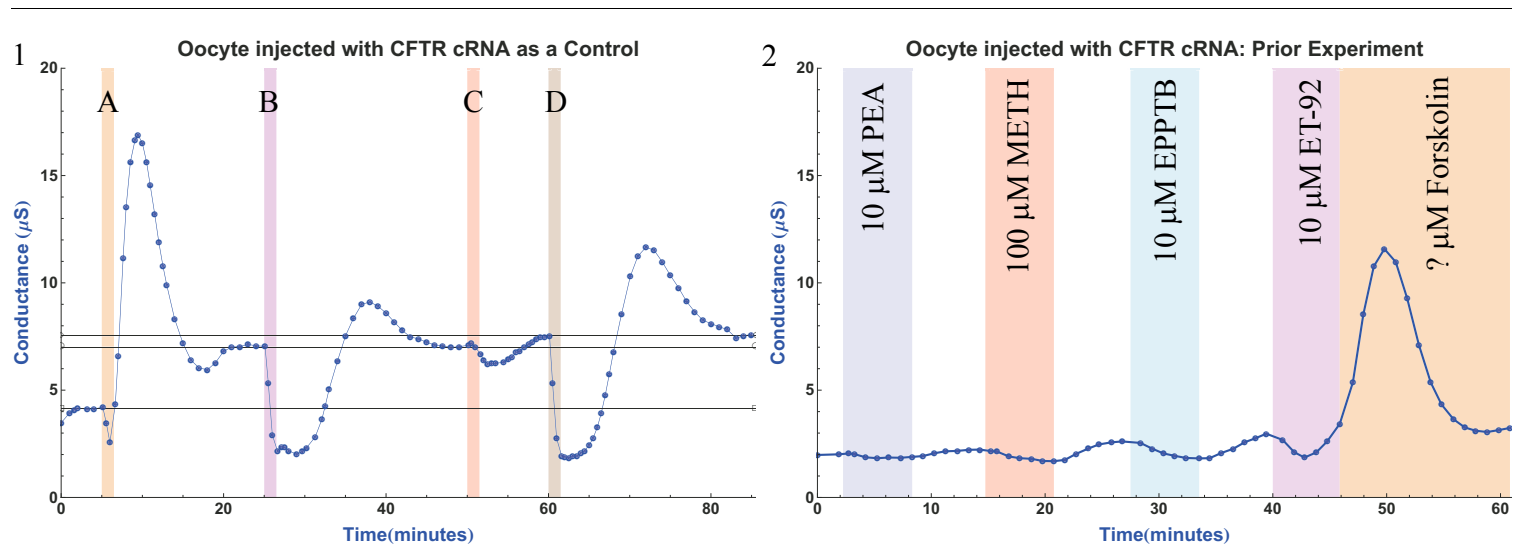
FIGURE 4.6(1) shows a control experiment done with an oocyte injected with hCFTR encoding RNA alone. Here $5 \mu \mathrm{M}$ forskolin was applied (A) then $10 \mu \mathrm{M}$ G5-109s8 (B). Upon seeing the drop in conductance after applying G5-109s8, 10 $\mu \mathrm{M}$ Meth was applied to demonstrate that a TAAR1/CFTR expressing oocyte had not been used by mistake. Finally a combination of G5-109s8 and forskolin was applied (D) resulting in a response between A and B. Overall, this oocyte had an unexpectedly weak response to forskolin and Meth depressed the conductance (maximum change in conductance $\approx-1 \mu \mathrm{S}$ ), which may signal a hitherto unknown target of Meth. The response to pulse D is difficult to explain as it does not look like a simple sum of response to A and $\mathrm{B}$. The response to $\mathrm{D}$ could indicate that G5-109s8 weakly inhibits $G_{\alpha s}$ or adenylyl cyclase. It could also indicate that G5109s8 affects a pathway involving CFTR.

Although the off-target drop in transmembrane conductance caused by G5109s8 (FIGURE 4.6(1)) needs to be confirmed, it is very troubling, as it is the result of unknown electrophysiological changes. Those changes could be detrimental to many systems, such as: the central nervous system, cardiovascular system and mucus membranes. Thus this off-target effect could be evidence for major or even fatal toxic side-effects such as: psychosis, heart failure and ulcers. The criteria of the FDA, and organizations like it around the world, to approve a new pharmaceutical agent is that the agent must be both efficacious and reasonably safe. In order to prove that $\mathbf{G 5 - 1 0 9 s 8}$ is reasonably safe and continue developing 


\section{Results}

the thyronamine family of compounds as pharmaceuticals, the cause of this off-target effect needs to be identified, evaluated for toxicity, and then new compounds in the thyronamine family would need to be screened against it. The identification of the mechanism for the electrophysiological off-target effect of G5-109s8 is a major biochemistry or chemical biology project and is beyond the scope of this drug discovery project.

With $\mathrm{n}=1$ for the control assay, it is reasonable for one to ask whether or not the off-target effect is experimentally reproducible, and whether or not the off-target effect is experimentally valid (or real, if one prefers). Reproducibility is an issue of how frequent a result occurs, whereas validity is an issue of experimental accuracy. Strictly as a point of logic, a valid result maybe difficult to reproduce due to low frequency of occurrence. If the off-target effect seen in FIGURE 4.6(1) is sufficiently infrequent, then G5-109s8 can be used in chemical biology studies of TAAR1 with few complications. However, for the purpose of drug discovery a compound's safety is a function of how frequently the compound causes adverse effects and their severity. Validating the off-target effect (again, off-target because TAAR1 was not present in the assay) could be complicated by the possibility that the oocytes have a heterogeneous (i.e.: poly-modal) response to G5-109s8 due to genetic variability of the oocytes where some of the modes are rare.

Assuming the off-target effect in FIGURE 4.6(1) is valid, it can not account for the depressed conductance seen in FIGURE 4.5F unless the off-target effect is the 


\section{Results}

result of inhibiting adenylyl cyclase, the G-proteins, or CFTR. If G5-109s8's only mode of action was through this off-target effect, and was completely independent of the TAAR1 pathway, then one would add the $10 \mu \mathrm{M}$ Meth response curve in FIGURE 4.5A (maximum change in conductance $\approx 70 \mu \mathrm{S}$ ) to the off-target effect (maximum change in conductance $\approx-5 \mu \mathrm{S}$ ) in order to get the response to a combination of $10 \mu \mathrm{M}$ Meth and $10 \mu \mathrm{M}$ G5-109s8. When that is done, the result is not FIGURE 4.5F. Therefore G5-109s8 must effect the TAAR1 pathway.

Upon seeing the G5-109s8 off-target effect, evidence was sought for a similar effect from ET-92. FIGURE 4.6(2) shows an experiment, done previously by the Grandy lab, dosing an oocyte injected with hCFTR encoding RNA alone. In the experiment, $10 \mu \mathrm{M}$ PEA, $100 \mu \mathrm{M}$ Meth, $10 \mu \mathrm{M}$ EPPTB, $10 \mu \mathrm{M}$ ET-92 and an unidentified concentration of forskolin were applied to the oocyte. In this experiment there appears to be a weak response to ET-92, maximum change in conductance $\approx-1 \mu \mathrm{S}$, and the possibility of very weak response to Meth and EPPTB. If this is not convincing, a comparison between the mouse in vivo data for ET-92/ vehicle and EPPTB/vehicle trials, FIGURE 1.6, clearly shows a dose-dependent drop in locomotion for ET-92/vehicle, especially from $5 \mathrm{mg} / \mathrm{kg}$ to $10 \mathrm{mg} / \mathrm{kg}$, but no such effect for the selective EPPTB. In addition to the ET-92 effect on locomotion, when ET-92 was first studied in vivo (mice) a $20 \mathrm{mg} / \mathrm{kg}$ dose of ET-92 caused the mice to be hypothermic for 4 days after injection, thus requiring the mice to be euthanized. ${ }^{82}$ Taken together, the possible electrophysiological observation (FIG- 
Results

URE 4.6(1)), thermoregulatory loss, and locomotion effects of the thyronamine family of analogs renders them a potentially risky class of compounds for further pharmaceutical development without first evaluating the actual risk posed by these effects.

\subsubsection{Testing the other compounds}

Since the original intent of making these compounds, G5-110s8 through G5114s5, was for use as intermediates in the synthesis of other compounds, not all of them were as pure as G5-109s8, but they were deemed sufficiently pure ( $>95 \%$ by ${ }^{1} \mathrm{H}-\mathrm{NMR}$ excluding residual solvents) for a first pass activity screen. The testing for G5-110s8 through G5-114s5 followed the procedure outlined in Chapter 7.2. FIGURE 4.8, and 4.11 do not all show the full forskolin response range because it is off the scale in comparison to the other peaks. FIGURE 4.10, a repeat of an earlier experiment, omitted repeating the $10 \mu \mathrm{M}$ forskolin and $10 \mu \mathrm{M}$ G5-113s5 pulse. None of the assayed compounds blocked forskolin activity.

All of the compounds show sub-basal transmembrane conductance for at least part of the response curve F. Only G5-114s5 (FIGURE 4.11) showed any reliable effect at $\leq 1 \mu \mathrm{M}$. G5-111s8 (FIGURE 4.8F) shows complete sub-basal conductance. G5-113s5 (FIGURE 4.10F) shows a peak followed by a sub-basal trough with the possibility of a brief sub-basal trough before the peak (this observed dip in conductance is small enough that it might be the result of an air bubble). G5110s8, G5-112s5 and G5-114s5 (FIGURE 4.7F, 4.9F, and 4.11E-F respectfully) 
show a sub-basal trough then peak followed by another sub-basal trough. The only way to produce a trough-peak-trough pattern in this system is if TAAR1 has basal activity, the compounds bind to TAAR1, and the compounds bind the ligand-free active form of TAAR1 faster than they bind the ligand-free inactive form of TAAR1 (this claim will be fully justified by kinetic analysis in Chapter 5). The peaks are the result of Meth stimulation, since Meth does not stimulate the G-proteins, adenylyl cyclase nor CFTR (FIGURE 4.6). If G5-110s8, G5-112s5 and G5$114 s 5$ inhibited the G-proteins, adenylyl cyclase or CFTR sufficiently to block the basal signal of TAAR1 then the TAAR1 Meth stimulated signal would also be blocked; therefore by elimination TAAR1 must be inhibited. Since all of the tested compounds are structurally very similar to G5-110s8, G5-112s5 and G5-114s5, it is logical to conclude by reason of similarity ${ }^{83}$ that their primary method of action is the inhibition of TAAR1 and not the inhibition of the G-proteins, adenylyl cyclase or CFTR. Since the compounds suppress TAAR1 activity to sub-basal levels, the compounds are inverse agonists. ${ }^{84}$ Going back to FIGURE 4.3, one might estimate that TAAR 1 basal activity is about $5 \%$ of its total possible activity (determined by dividing the basal level by the maximum Meth response). Using that estimation all the compounds are around $\mathrm{IC}_{90}$ at $10 \mu \mathrm{M}$, otherwise there would be enough TAAR1 available for Meth to bind for the total activity to remain above the baseline. More justification for this claim will be provided in the next chapter. 


\section{Results}

FIGURE 4.7: Heads up competition between Meth and G5-110s8 $(n=1)$. Pulses were 1.5 minutes in duration. A) $10 \mu \mathrm{M}$ Meth, B) $10 \mu \mathrm{M}$ Meth $\& 1 \mathrm{nM}$ G5-110s8, C) $10 \mu \mathrm{M}$ Meth $\& 10 \mathrm{nM}$ G5-110s8, D) 10 $\mu \mathrm{M}$ Meth $\& 100 \mathrm{nM}$ G5-110s8, E) $10 \mu \mathrm{M}$ Meth $\& 1 \mu \mathrm{M}$ G5-110s8, F) $10 \mu \mathrm{M}$ Meth $\& 10 \mu \mathrm{M}$ G5-110s8, G) $10 \mu \mathrm{M}$ G5-110s8 \& $10 \mu \mathrm{M}$ forskolin. Grey line segments are the approximate baseline.

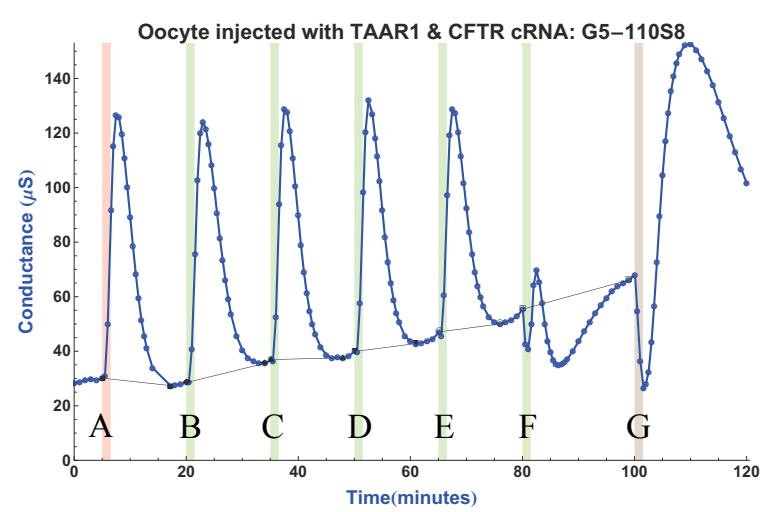

FIGURE 4.8: Heads up competition between Meth and G5-111s8 $(\mathrm{n}=1)$. Pulses were 1.5 minutes in duration. A) $10 \mu \mathrm{M}$ Meth, B) $10 \mu \mathrm{M}$ Meth \& $1 \mathrm{nM}$ G5-111s8, C) $10 \mu \mathrm{M}$ Meth \& $10 \mathrm{nM}$ G5-111s8, D) 10 $\mu \mathrm{M}$ Meth $\& 100 \mathrm{nM}$ G5-111s8, E) $10 \mu \mathrm{M}$ Meth $\& 1 \mu \mathrm{M}$ G5-111s8, F) $10 \mu \mathrm{M}$ Meth $\& 10 \mu \mathrm{M}$ G5-111s8, G) $10 \mu \mathrm{M}$ G5-111s8 \& $10 \mu \mathrm{M}$ forskolin (off-scale). Grey line segments are the approximate baseline.

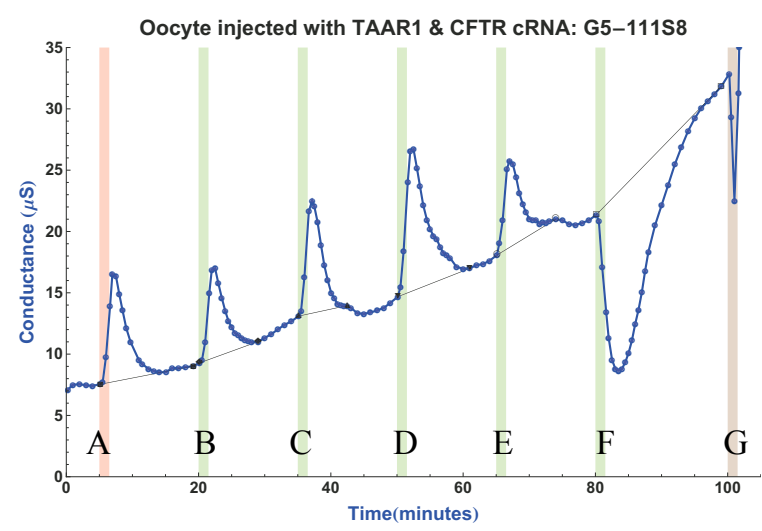




\section{Results}

FIGURE 4.9: Heads up competition between Meth and G5-112s5 ( $\mathrm{n}=1$ ). Pulses were 1.5 minutes in duration. A) $10 \mu \mathrm{M}$ Meth, B) $10 \mu \mathrm{M}$ Meth \& $1 \mathrm{nM}$ G5-112s5, C) $10 \mu \mathrm{M}$ Meth $\& 10 \mathrm{nM}$ G5-112s5, D) 10 $\mu \mathrm{M}$ Meth $\& 100 \mathrm{nM}$ G5-112s5, E) $10 \mu \mathrm{M}$ Meth $\& 1 \mu \mathrm{M}$ G5-112s5, F) $10 \mu \mathrm{M}$ Meth $\& 10 \mu \mathrm{M}$ G5-112s5, G) $10 \mu \mathrm{M}$ G5-112s5 \& $10 \mu \mathrm{M}$ forskolin. Grey line segments are the approximate baseline.

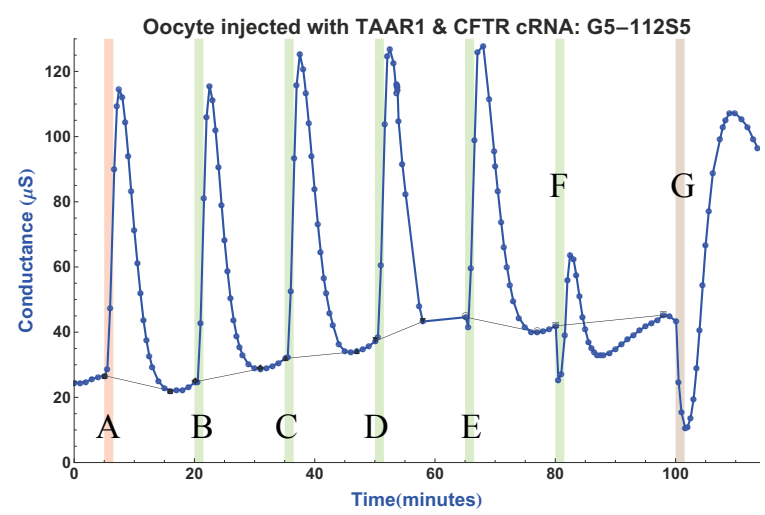

FIGURE 4.10: Heads up competition between Meth and G5-113s5 ( $\mathrm{n}=1)$. Pulses were 1.5 minutes in duration. A) $10 \mu \mathrm{M}$ Meth, B) $10 \mu \mathrm{M}$ Meth \& $1 \mathrm{nM}$ G5-113s5, C) $10 \mu \mathrm{M}$ Meth \& $10 \mathrm{nM}$ G5-113s5, D) 10 $\mu \mathrm{M}$ Meth $\& 100 \mathrm{nM}$ G5-113s5, E) $10 \mu \mathrm{M}$ Meth $\& 1 \mu \mathrm{M}$ G5-113s5, F) $10 \mu \mathrm{M}$ Meth $\& 10 \mu \mathrm{M}$ G5-113s5. Grey line segments are the approximate baseline.

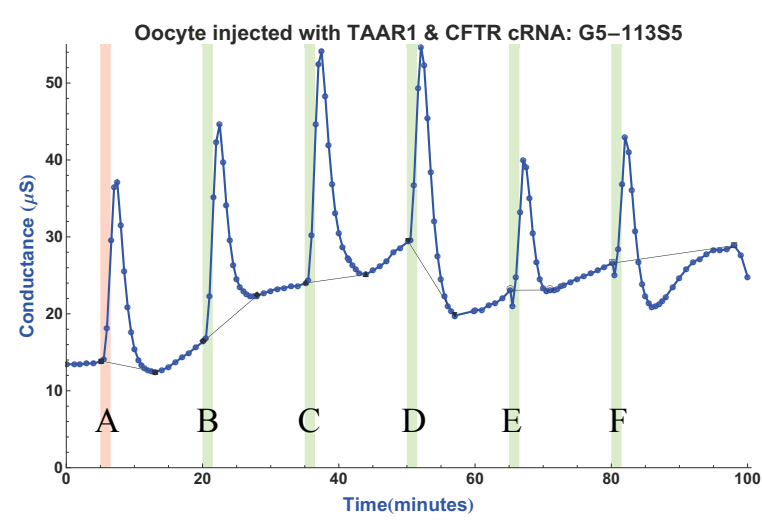




\section{Concluding remarks about the assays}

FIGURE 4.11: Heads up competition between Meth and G5-114s5 ( $\mathrm{n}=1)$. Pulses were 1.5 minutes in duration. A) $10 \mu \mathrm{M}$ Meth, B) $10 \mu \mathrm{M}$ Meth \& $1 \mathrm{nM}$ G5-114s5, C) $10 \mu \mathrm{M}$ Meth \& $10 \mathrm{nM}$ G5-114s5, D) 10 $\mu \mathrm{M}$ Meth $\& 100 \mathrm{nM}$ G5-114s5, E) $10 \mu \mathrm{M}$ Meth $\& 1 \mu \mathrm{M}$ G5-114s5, F) $10 \mu \mathrm{M}$ Meth $\& 10 \mu \mathrm{M}$ G5-114s5, G) $10 \mu \mathrm{M}$ G5-114s5 \& $10 \mu \mathrm{M}$ forskolin (off-scale). Grey line segments are the approximate baseline.

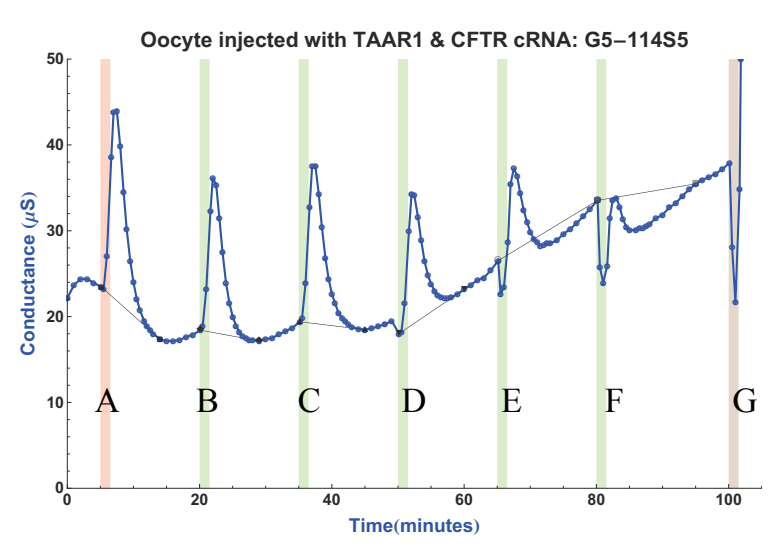

\subsection{Concluding remarks about the assays}

Although one can draw conclusions from these assay results, it would be best to repeat the experiment at least 3 times with oocytes that express hTAAR 1 and hCFTR more consistently then the ones used here. Doing so would both verify the results and allow quantification. Having to make decisions based on minimal, poor quality or incomplete data is not uncommon in industrial research, where the focus is on following up on promising leads and quickly moving away from unfavorable ones. That said, one tries to be as thorough as possible. The industrial research model is not prefect; false leads get followed and promising ones accidentally get discarded. Also, industrial research can seldom afford to treat every lead as thoroughly as is often done in academic research. In the end, industrial research must produce a marketable product. Although done in an academic setting, the assaying and subsequent analysis follow the industrial research model. At this time, the thy- 


\section{Concluding remarks about the assays}

ronamine family of compounds appear to be high risk for further pharmaceutical

development. 
Starting from first principles, ${ }^{85}$ a series of kinetic models were developed to explain the observed biological data in Chapter 4 .

\section{$5.1 \quad$ Quick review of kinetics}

Unimolecular kinetics ( $\mathrm{A} \rightarrow \mathrm{B}$, intramolecular conformation changes, disassociations, radioactive decay, etc.) are modeled by equations in the form of

$$
\frac{\mathrm{d}}{\mathrm{d} t}[\chi]=k[\chi]
$$

and bimolecular kinetics ( $\mathrm{A}+\mathrm{B} \rightarrow \mathrm{C}$, reactions between two chemicals, etc.) are modeled by equations in the form of

$$
\frac{\mathrm{d}}{\mathrm{d} t}[\chi]=k[\chi][\Psi]
$$

In EQ 5.1 - 5.2, $[\chi]$ is the concentration or activity of $\chi$ (similarly for $\Psi$ ), and $k$ is the rate constant (positive when creating a chemical species and negative when destroying a chemical species). To model more complex systems, one builds rate equations by adding terms for each reaction that occurs in the system. Each chemical species involved in the system requires its own rate equation. Also, mass balance equations and initial conditions are needed, both of which serve as constraints on the system. For example, EQ 5.4 - 5.7 are the complete set of equations needed 


\section{TAAR1 Kinetics}

to model a simple irreversible bimolecular reaction (EQ 5.3) with initial concentrations given by $\mathrm{A}_{0}, \mathrm{~B}_{0}$, and $\mathrm{C}_{0}$.

$$
\begin{gathered}
\mathrm{A}+\mathrm{B} \rightarrow \mathrm{C} \\
{[\mathrm{A}]=\mathrm{A}_{0}-[\mathrm{C}]} \\
{[\mathrm{B}]=\mathrm{B}_{0}-[\mathrm{C}]} \\
\mathrm{C}_{0}=0 \\
-\frac{\mathrm{d}}{\mathrm{d} t}[\mathrm{~A}]=-\frac{\mathrm{d}}{\mathrm{d} t}[\mathrm{~B}]=\frac{\mathrm{d}}{\mathrm{d} t}[\mathrm{C}]=k[\mathrm{~A}][\mathrm{B}]=k\left[\mathrm{~A}_{0}-\mathrm{C}\right]\left[\mathrm{B}_{0}-\mathrm{C}\right]
\end{gathered}
$$

Since this example is a simple single step reaction, one only needs to solve for [C] and then plug-in [C] into EQ 5.4 - 5.5 to get [A], and [B].

\section{$5.2 \quad T A A R 1$ Kinetics}

Receptors with basal activity, such as TAAR1, exist in an equilibrium between a ligand-free active form and a ligand-free inactive form. The interconversion between the two forms is a unimolecular process. Once ligands are introduced to the receptor, the ligands could potentially bind to either ligand-free form of the receptor creating either an active ligand-receptor complex or an inactive ligandreceptor complex. The ligand and the receptor bind in a bimolecular process. Interconversion between the active ligand-receptor complex and the inactive ligandreceptor complex is possible (a unimolecular process). Lastly, both forms of the ligand-receptor complex can disassociate in a unimolecular process. 


\section{TAAR1 Kinetics}

FIGURE 5.1A shows all possible pathways for TAAR1 to interact with Meth (an agonist) and an inhibitor [as short-hand, inhibitor = inverse agonist]. Such a model has 22 rate constants, and with enough work one probably could make such a model fit most data. FIGURE 5.1B shows a simplified kinetic scheme where the inhibitor-TAAR1 complex can only exist in an inactive state $\left(\mathrm{IR}_{\mathrm{i}}\right)$ and Meth only forms an active Meth-TAAR1 complex $\left(\mathrm{MR}_{\mathrm{a}}\right)$. In FIGURE 5.1C, the equilibrium between the ligand-free inactive receptor $\left(R_{i}\right)$ and its active form $\left(R_{a}\right)$ is extremely fast compared to all other rate constants. Model C is also equivalent to Model B when $\mathrm{k}_{3}=\mathrm{k}_{2}, \mathrm{k}_{-3}=\mathrm{k}_{-2}, \mathrm{k}_{4}=\mathrm{k}_{5}, \mathrm{k}_{-4}=\mathrm{k}_{-5}$ and the equilibrium between $\mathrm{R}_{\mathrm{i}}$ and $\mathrm{R}_{\mathrm{a}}$ is very slow compared to all other processes in the model. Included in Model C is a gross simplification leading to the production of cAMP followed by the activation of CFTR. In all three models, arrows indicating either agonist/inhibitor binding or disassociation have been omitted for clarity. 


\section{TAAR1 Kinetics}

FIGURE 5.1: Kinetic Schemes for TAAR1: A) All possible pathways for the basally active TAAR1 to interact with Methamphetamine (Meth) and an Inhibitor. The 22 rate constants have been omitted for clarity as have the arrows for binding and unbinding of both Meth and the Inhibitor. B) Simplified scheme assuming the Meth-TAAR1 complex is always active and the Inhibitor-TAAR1 complex is always inactive. C) When $\mathrm{k}_{1}$ and $\mathrm{k}_{-1}$ are much faster than any of the other rate constants, then the total ligand-free TAAR1 can be treated as a single pool with active and inactive forms held at a fixed ratio. A step for the formation of cAMP and another for the CFTR cycle were added to model the oocyte data. Original artwork by Troy Wahl.

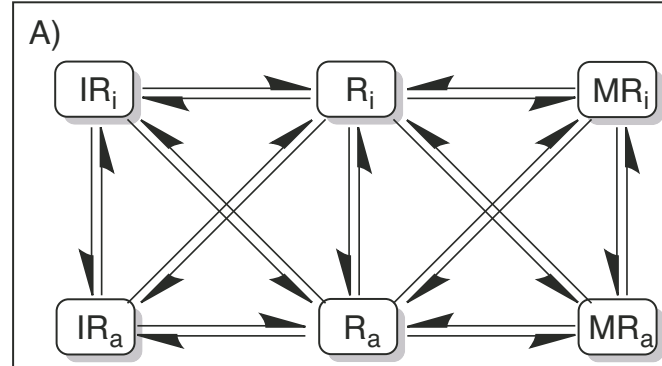

C)

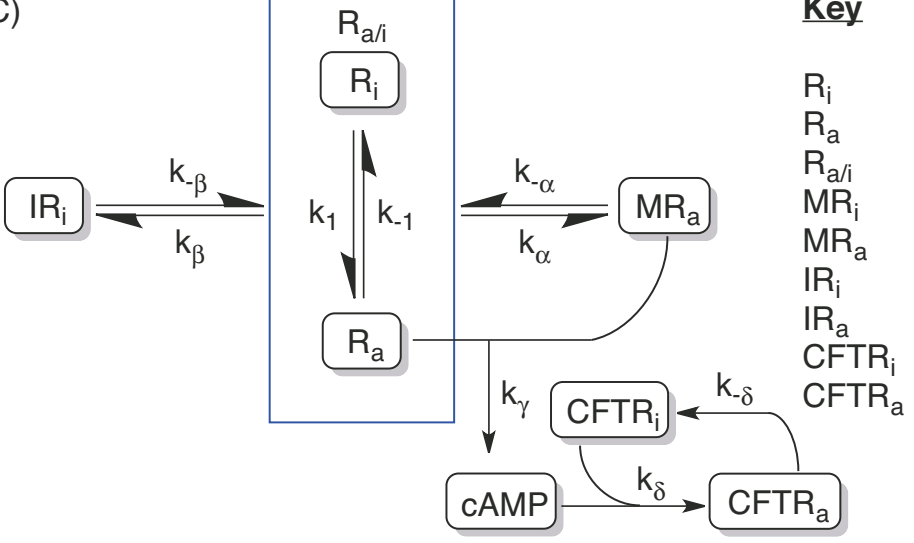

B)

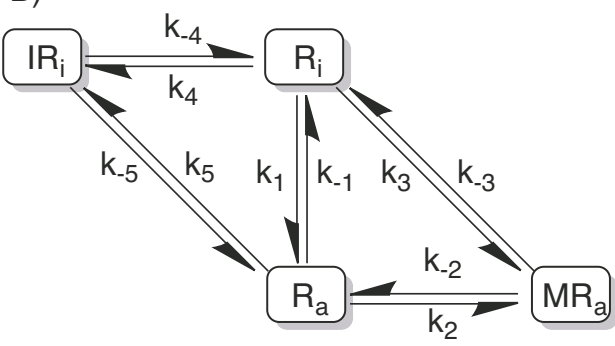

Key

Inactive Ligand-Free TAAR1

Active Ligand-Free TAAR1

Total Ligand-Free TAAR1

Inactive Meth-TAAR1 Complex

Active Meth-TAAR1 Complex

Inactive Inhibitor-TAAR1

Active Inhibitor-TAAR1

Inactive CFTR

Active CFTR

When one is trying to fit a reaction kinetic model to data, it is vital to have

either accurate concentration or activity data for all chemical species in the model.

Since neither the TAAR1 nor the CFTR expression levels were available nor were

they consistent, qualitative agreement between the models and the data was

sought. If the oocytes had had consistent hTAAR1 and hCFTR expression levels,

then the total concentration of each could have been set to 1, allowing the rate con-

stants to act as scaling factors, and the data fit. Once the data was fit, the rate con-

stants could have been used to calculate $\mathrm{IC}_{50}$ values for each assayed compound. 
Model B: Modeling TAAR1 equilibria

In all the simulations, the rate constants $\mathrm{k}_{1}$ and $\mathrm{k}_{-1}$ were chosen to produce an $\mathrm{R}_{\mathrm{a}}: \mathrm{R}_{\mathrm{i}}$ ratio of $1: 19$ at equilibrium. Also, the concentrations for TAAR1, Meth and inhibitor used in the models are arbitrary. As long as the rate constant are scaled inversely, the concentrations can be changed without changing the results.

\subsection{Model B: Modeling TAAR1 equilibria}

Due to the kinetic complexity of basally active systems, the kinetic models were used to explore how TAAR1 might behave by varying rate constants and receptor concentration. The later variation could reveal some potential complications in interpreting in vivo overexpression studies. For the purpose of modeling the binding kinetics of Meth to TAAR1, in vivo experiments where Meth is administered to mice, the mice can be approximated as a closed system since the biological half-life (metabolism and excretion) of Meth is approximately 13 hours yet the effects of Meth on mice are noticeable within minutes. Chemical reactions in closed systems result in equilibria and are simpler to model than ones in open systems, so a TAAR1 equilibrium model will be the first one examined.

Using the kinetic scheme shown in FIGURE 5.1B, the mass balance equations for a closed system are given by EQ $5.8-\mathbf{5 . 1 0}$, and the rate equations are listed in EQ $5.11-5.13$

$$
\begin{gathered}
{\left[R_{T}\right]=\left[R_{i}\right]+\left[R_{a}\right]+\left[M R_{a}\right]+\left[I R_{i}\right]} \\
{\left[M_{T}\right]=[M]+\left[M R_{a}\right]}
\end{gathered}
$$


Model B: Modeling TAAR1 equilibria

$$
\left[\operatorname{Inh}_{T}\right]=[\operatorname{Inh}]+\left[I R_{i}\right]
$$

(EQ 5.10)

$$
\begin{aligned}
\frac{\mathrm{d}}{\mathrm{d} t}\left[R_{a}\right]= & k_{1}\left[R_{i}\right]-\left(k_{-1}+k_{2}[M]+k_{5}[\operatorname{Inh}]\right)\left[R_{a}\right]+k_{-2}\left[M R_{a}\right]+k_{-5}\left[I R_{i}\right] \\
= & k_{1}\left(\left[R_{T}\right]-\left[R_{a}\right]-\left[M R_{a}\right]-\left[I R_{i}\right]\right)-\left(k_{-1}+k_{2}\left(\left[M_{T}\right]-\left[M R_{a}\right]\right)+k_{5}\left(\left[I n h_{T}\right]-\left[I R_{i}\right]\right)\right)\left[R_{a}\right] \\
& +k_{-2}\left[M R_{a}\right]+k_{-5}\left[I R_{i}\right] \\
\frac{\mathrm{d}}{\mathrm{d} t}\left[M R_{a}\right]= & \left(k_{3}\left[R_{i}\right]+k_{2}\left[R_{a}\right]\right)[M]-\left(k_{-2}+k_{-3}\right)\left[M R_{a}\right] \\
= & \left(k_{3}\left(\left[R_{T}\right]-\left[R_{a}\right]-\left[M R_{a}\right]-\left[I R_{i}\right]\right)+k_{2}\left[R_{a}\right]\right)\left(\left[M_{T}\right]-\left[M R_{a}\right]\right)-\left(k_{-2}+k_{-3}\right)\left[M R_{a}\right] \\
\frac{\mathrm{d}}{\mathrm{d} t}\left[I R_{i}\right]= & \left(k_{4}\left[R_{i}\right]+k_{5}\left[R_{a}\right]\right)[I n h]-\left(k_{-4}+k_{-5}\right)\left[I R_{i}\right] \\
= & \left(k_{4}\left(\left[R_{T}\right]-\left[R_{a}\right]-\left[M R_{a}\right]-\left[I R_{i}\right]\right)+k_{5}\left[R_{a}\right]\right)\left(\left[I n h_{T}\right]-\left[I R_{i}\right]\right)-\left(k_{-4}+k_{-5}\right)\left[I R_{i}\right]
\end{aligned}
$$

The equations form a non-linear system of differential equations that does not have a closed form analytical solution, so numerical integration is needed to solve them. The equations were programed into Mathematica using the Runge-Kutta numerical method (this is not a built in function) with a step size, $\mathrm{h}$, of $10^{-4} \mathrm{~min}$ utes. Runge-Kutta has an error in calculation on the order of $\mathrm{h}^{5}$, so at that step size the calculations had an error on the order of $10^{-20}$, which was smaller then the precision of the calculations ( $\sim 16$ digits). Thus round-off error was the largest source of error in the simulations, being on the order of $10^{-16}$ times the number of steps need to get to a particular time point in the simulation. Since the simulations used a maximum of 200,000 steps, the maximum error in the simulations was on the order of $10^{-10}$. 


\section{Model B: Modeling TAAR1 equilibria}

FIGURE 5.2: The model is very sensitive to rate of equilibrium between $R_{i}$ and $R_{a}$. The only difference between $\mathrm{A}$ and $\mathrm{B}$ are the values of $\mathrm{k}_{1}$ and $\mathrm{k}_{-1}$, with the values in $\mathbf{A} 5$ times those in $\mathbf{B}$. These simulations show the expected effects of Meth, an agonist, in that total TAAR1 activity increases above the basal activity. Due to the slow equilibrium between $R_{i}$ and $R_{a}$, the disassociation of the Meth-TAAR1 complex leads to an increase in active ligand-free TAAR1 $\left(\mathrm{R}_{\mathrm{a}}\right)$, which is the opposite of effect seen in $\mathbf{A}$.
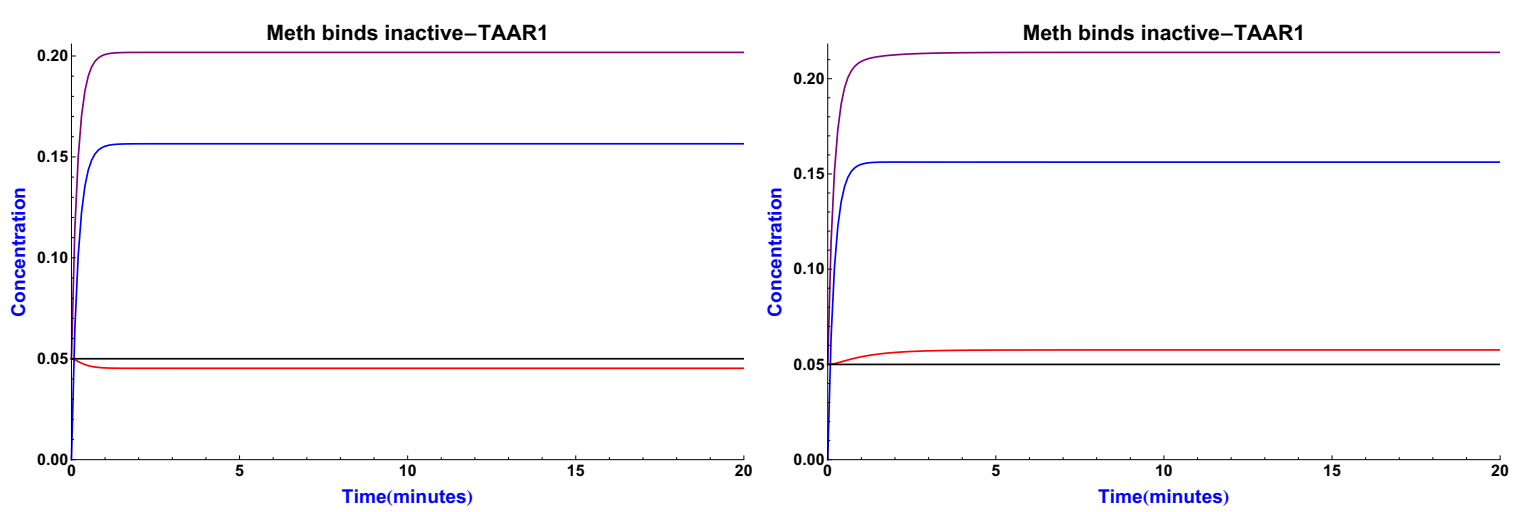

$\begin{array}{lll}\text { Active Ligand-Free TAAR1 } & \text { Total Active TAAR1 } \\ \text { Active Meth-TAAR1 Complex } & \text { Basal TAAR1 Activity } \\ \text { Total TAAR1 }=1 \mathrm{M} & k_{1}=0.25 & k_{-1}=4.75 \\ \text { Total Meth }=0.18 \mathrm{M} & k_{2}=0.15 & k_{-2}=0.1 \\ \text { Total Inhibitor }=0 \mathrm{M} & k_{3}=5 & k_{-3}=0.5 \\ & k_{4}=0 & k_{-4}=0 \\ & k_{5}=0 & k_{-5}=0\end{array}$

FIGURE 5.2 shows the simulation of Meth binding to TAAR1. The only difference between simulation $\mathbf{A}$ and $\mathbf{B}$ is that $K_{1}$ and $K_{-1}$ are 5 times faster in FIGURE

5.2A then in FIGURE 5.2B. By slowing the rate at which $R_{a}$ and $R_{i}$ reach equilibrium, the concentrations active ligand-free TAAR1 $\left(R_{a}\right)$, and total active TAAR1 increase compared to the faster equilibrium in FIGURE 5.2A. This is the result of the Meth-TAAR1 complex $\left(\mathrm{MR}_{\mathrm{a}}\right)$ disassociation rate into $\mathrm{R}_{\mathrm{a}}$, determined by $\mathrm{k}_{-2}$, causing $R_{a}$ to accumulate faster than $R_{a}$ can be converted into the inactive form of TAAR1, $\mathrm{R}_{\mathrm{i}}$. 


\section{Model B: Modeling TAAR1 equilibria}

When Meth and Inhibitor are added to TAAR1, it is not difficult to suppress

total TAAR1 activity below its basal level, FIGURE 5.3. In order to do so, $\mathrm{k}_{4}$ and

$\mathrm{k}_{5}$ (the inhibitor rate binding constants) must be much faster than the rate binding constants for Meth, $\mathrm{k}_{2}$ and $\mathrm{k}_{3}$. This should not be surprising as it only takes a little Meth activation to increase the total activity of TAAR1 above the basal level, but the inhibitor must block nearly all of TAAR1 to suppress the activity of the basal level. Also, in the case of a slow equilibrium between $\mathrm{R}_{\mathrm{i}}$ and $\mathrm{R}_{\mathrm{a}}$, the inhibitor must rapidly bind $\mathrm{R}_{\mathrm{a}}$ to produce a rapid drop in total activity below the basal level.

When the $R_{i}$ and $R_{a}$ equilibrium is fast, a drop in $\left[R_{i}\right]$ is quickly matched by a drop in $\left[R_{a}\right]$.

FIGURE 5.3: Simulation of competition between Meth and Inhibitor. It is fairly easy to suppress TAAR1 below its basal activity, but to do so the inhibitor must bind to both inactive TAAR1 $\left(R_{i}\right)$ and active TAAR1 $\left(R_{a}\right)$ much faster than Meth.

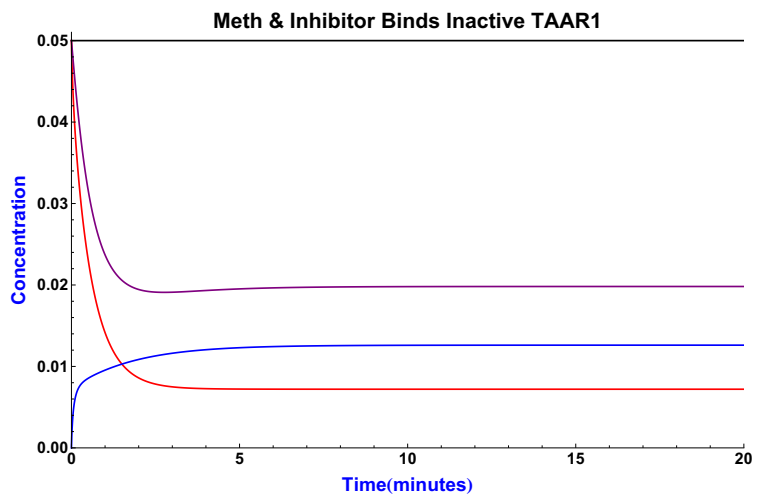

$$
\begin{array}{lll}
\text { Active Ligand-Free TAAR1 } & \text { Total Active TAAR1 } \\
\text { Active Meth-TAAR1 Complex } & \text { Basal TAAR1 Activity } \\
\text { Total TAAR1 }=1 \mathrm{M} & k_{1}=0.05 & k_{-1}=0.95 \\
\text { Total Meth }=0.18 \mathrm{M} & k_{2}=0.05 & k_{-2}=0.01 \\
\text { Total Inhibitor }=1.1 \mathrm{M} & k_{3}=1 & k_{-3}=0.5 \\
& k_{4}=40 & k_{-4}=0.25 \\
& k_{5}=4 & k_{-5}=0.01
\end{array}
$$

A counter-intuitive result is shown in FIGURE 5.4B, where an "overexpression" of TAAR1, simulated by the use of 4 M TAAR1 instead of the $1 \mathrm{M}$ used in FIGURE 5.4A, results in Meth causing a drop in total activity. For the simulations 
Model B: Modeling TAAR1 equilibria

in FIGURE 5.4, the rate constants have been set so that the fastest reactions proceeds from Meth binding the active form of TAAR1 $\left(\mathrm{R}_{\mathrm{a}}\right)$, to the Meth-TAAR1 complex disassociating into the inactive form of TAAR1 $\left(\mathrm{R}_{\mathrm{i}}\right)$, resulting in the accumulation of $R_{i}$ due of the slow equilibrium between $R_{i}$ and $R_{a}$. In the nonoverexpression case, FIGURE 5.4A, despite the fact $R_{a}$ is depleted, there is enough unbound Meth to create an equilibrium between Meth and $\mathrm{MR}_{\mathbf{a}}$ that results in a net increase of TAAR1 activity. That is not the case with the TAAR1 overexpression, FIGURE 5.4B. In Revel's in vivo study of TAAR1 overexpressing mice, ${ }^{86}$ conclusions are drawn based on the fact that Meth does not produce much stimulation in the overexpressing mice, however the result shown in FIGURE 5.4B requires extra caution when considering those conclusions. 


\section{Model C: Modeling the oocyte data}

FIGURE 5.4: Receptor overexpression can lead to counter-intuitive results when the receptor has basal activity. The only difference between $\mathbf{A}$ and $\mathbf{B}$ is the concentration of TAAR1. In this example, the fastest path is Meth binding to active TAAR1 $\left(\mathrm{R}_{\mathbf{a}}\right)$ to form the active Meth-TAAR1 complex $\left(\mathrm{MR}_{\mathbf{a}}\right)$ which in turn releases Meth and inactive TAAR1 $\left(\mathrm{R}_{\mathbf{i}}\right)$. (A) Although $\mathrm{R}_{\mathbf{a}}$ is depleted, there is enough unbound Meth to create an equilibrium between Meth and $\mathrm{MR}_{\mathbf{a}}$ that results in a net increase of TAAR1 activity. That is not the case with the TAAR1 overexpression, B.
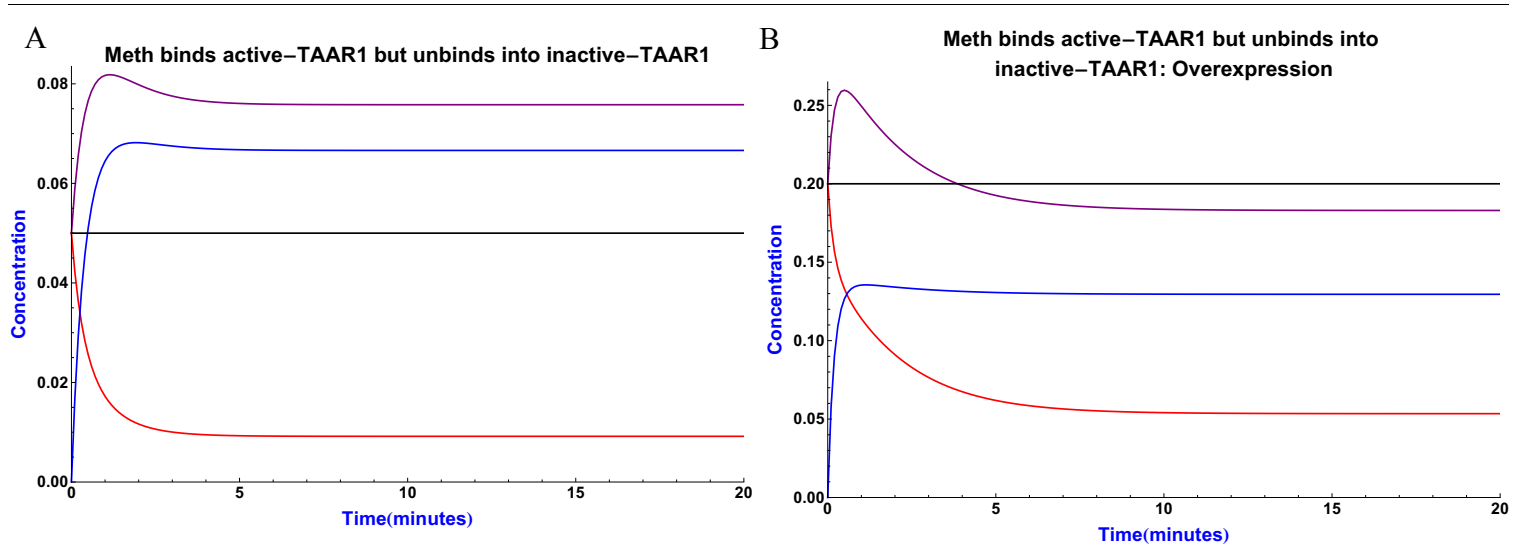

$$
\begin{array}{lll}
\text { Active Ligand-Free TAAR1 } & \text { Total Active TAAR1 } \\
\text { Active Meth-TAAR1 Complex } & \text { Basal TAAR1 Activity } \\
\text { Total TAAR1 }=1 \mathrm{M} & k_{1}=0.005 & k_{-1}=0.095 \\
\text { Total Meth }=0.18 \mathrm{M} & k_{2}=10 & k_{-2}=0.1 \\
\text { Total Inhibitor }=0 \mathrm{M} & k_{3}=0.6 & k_{-3}=1 \\
& k_{4}=0 & k_{-4}=0 \\
& k_{5}=0 & k_{-5}=0
\end{array}
$$

\begin{tabular}{|lll|}
\hline Active Ligand-Free TAAR1 & Total Active TAAR1 \\
Active Meth-TAAR1 Complex & Basal TAAR1 Activity \\
Total TAAR1 $=4 \mathrm{M}$ & $k_{1}=0.005$ & $k_{-1}=0.095$ \\
Total Meth $=0.18 \mathrm{M}$ & $k_{2}=10$ & $k_{-2}=0.1$ \\
Total Inhibitor $=0 \mathrm{M}$ & $k_{3}=0.6$ & $k_{-3}=1$ \\
& $k_{4}=0$ & $k_{-4}=0$ \\
& $k_{5}=0$ & $k_{-5}=0$ \\
\hline
\end{tabular}

\subsection{Model C: Modeling the oocyte data}

In order to model the oocyte data, one must address the fact that the experiments were not closed systems as the solutions with the test compounds flowed to the oocytes, where the oocytes respond to some of the molecules while the remainder flows past, never to interact with the oocyte again. Likewise, when the oocyte releases a molecule into the solution, that molecule is washed away. Model B could be used, but the mass balance equations for both Meth and the inhibitor would have to keep track of how many molecules of each compound flowed past the oocyte, and how much was bound to TAAR1, otherwise model would lack suf- 


\section{Model C: Modeling the oocyte data}

ficient constraints to work properly. To do that, one would have to accurately know the quantities, in moles, of each chemical species present in the model, which was not the case. Fortunately, there is a work-around. While the test compounds are being applied, the incoming stream has a constant concentration and one can treat the oocyte as a point or infinitely thin membrane in the flow. This approximation allows one to disregard the molecules that either flowed past or were released by the oocyte. With this approximation, the mass balance equations are

$$
\begin{gathered}
1=\left[R_{a / i}\right]+\left[M R_{a}\right]+\left[I R_{i}\right] \\
{[M]=\left\{\begin{array}{cc}
0 & t<0 \\
1 & 0 \leq t<1.5 \\
0 & 1.5 \leq t
\end{array}\right.} \\
{[\text { Inh }]=\left\{\begin{array}{cc}
0 & t<0 \\
1 & 0 \leq t<1.5 \\
0 & 1.5 \leq t
\end{array}\right.}
\end{gathered}
$$

Before the model can be constructed, the rate of equilibrium between $R_{i}$ and $R_{a}$ must be set either much faster or much slower (with $\mathrm{k}_{3}=\mathrm{k}_{2}, \mathrm{k}_{-3}=\mathrm{k}_{-2}, \mathrm{k}_{4}=\mathrm{k}_{5}$, and $\mathrm{k}_{-4}=\mathrm{k}_{-5}$ ) than any other process in the model. Doing this converts Model B into the TAAR1 portion of Model C. A very rapid equilibrium between $R_{i}$ and $R_{a}$ means that no matter what the other rate constants are, the ratio between $\left[\mathrm{R}_{\mathrm{i}}\right]$ and $\left[R_{a}\right]$ will be constant. With the other extreme, the system decouples into two linearly independent systems. With the equilibrium between $R_{i}$ and $R_{a}$ set to be very fast, one only need to keep track of the total amount of ligand-free TAAR $1, \mathrm{R}_{\mathrm{a} / \mathrm{i}}$, 


\section{Model C: Modeling the oocyte data}

during the simulation then, afterwards, partition out $R_{i}$ and $R_{a}$ according to $E \mathbf{Q}$

\subsection{7 - 5.18}

$$
\begin{gathered}
{\left[R_{a}\right]=\frac{k_{1}\left[R_{a / i}\right]}{k_{1}+k_{-1}}} \\
{\left[R_{i}\right]=\frac{k_{-1}\left[R_{a / i}\right]}{k_{1}+k_{-1}}} \\
{\left[M R_{a}\right]_{t=0}=\left[I R_{i}\right]_{t=0}=0} \\
{[C A M P]_{t=0}=\frac{k_{1} k_{\gamma} k_{-\delta}}{k_{-\delta}\left(k_{1}+k_{-1}\right)-k_{1} k_{\gamma} k_{\delta}}} \\
{[C F T R]_{t=0}=\frac{k_{1} k_{\gamma} k_{\delta}}{k_{-\delta}\left(k_{1}+k_{-1}\right)}}
\end{gathered}
$$

In order to couple TAAR1 to CFTR, the model must produce cAMP which in turn activates CFTR. Model C (FIGURE 5.1C) treats cAMP production as an unimolecular process dependent on the concentration of all active TAAR1 species. In reality, the production of cAMP is the result of a sequence of reactions; however, for a crude qualitative model, the treatment is sufficient. Although it might be tempting to eliminate cAMP from the model by having active TAAR1 directly drive CFTR, doing so would be a mistake as cAMP serves as a capacitor, both creating a time delay and smoothing out sharp transitions. Putting this all together with the initial conditions, EQ 5.19 - 5.21, the rate equations for Model C are

$$
\begin{aligned}
\frac{\mathrm{d}}{\mathrm{d} t}\left[M R_{a}\right] & =k_{\alpha}[M]\left[R_{a / i}\right]-k_{-\alpha}\left[M R_{a}\right] \\
& =k_{\alpha}[M]\left(1-\left[M R_{a}\right]-\left[I R_{i}\right]\right)-k_{-\alpha}\left[M R_{a}\right] \\
\frac{\mathrm{d}}{\mathrm{d} t}\left[I R_{i}\right] & =k_{\beta}[\operatorname{Inh}]\left[R_{a / i}\right]-k_{-\beta}\left[I R_{i}\right] \\
& =k_{\beta}[\operatorname{Inh}]\left(1-\left[M R_{a}\right]-\left[I R_{i}\right]\right)-k_{-\beta}\left[I R_{i}\right]
\end{aligned}
$$


Model C: Modeling the oocyte data

$$
\begin{aligned}
\frac{\mathrm{d}}{\mathrm{d} t}[\operatorname{cAMP}] & =k_{\gamma}\left(\left[M R_{a}\right]+\left[R_{a}\right]\right)-k_{\delta}[\text { CAMP }]\left[C F T R_{i}\right] \\
& =k_{\gamma}\left(\left[M R_{a}\right]+\frac{k_{1}\left[R_{a / i}\right]}{k_{1}+k_{-1}}\right)-k_{\delta}[c A M P]\left(1-\left[C F T R_{a}\right]\right) \\
& =k_{\gamma}\left(\left[M R_{a}\right]+\frac{k_{1}\left(1-\left[M R_{a}\right]-\left[I R_{i}\right]\right)}{k_{1}+k_{-1}}\right)-k_{\delta}[c A M P]\left(1-\left[C F T R_{a}\right]\right) \\
& =k_{\gamma}\left(k_{-1}\left[M R_{a}\right]+k_{1}\left(1-\left[I R_{i}\right]\right)\right)-k_{\delta}[c A M P]\left(1-\left[C F T R_{a}\right]\right) \\
\frac{\mathrm{d}}{\mathrm{d} t}\left[C F T R_{a}\right] & =k_{\delta}[c A M P]\left[C F T R_{i}\right]-k_{-\delta}\left[C F T R_{a}\right] \\
& =k_{\delta}[C A M P]\left(1-\left[\operatorname{CFTR}_{a}\right]\right)-k_{-\delta}\left[C F T R_{a}\right]
\end{aligned}
$$

Instead of using concentrations in this model, the relative activities of the compounds will be used thus forcing the rate constants to contain information about the maximum concentration of the compounds. A convenient feature of Model C is that the rate equations for the TAAR1 portion, EQ 5.22 - 5.23, have exact solutions, though they are very long. In order to calculate the values for cAMP and CFTR, EQ 5.24 - 5.25, one takes the exact solutions for $\mathrm{MR}_{\mathrm{a}}$ and $\mathrm{IR}_{\mathrm{i}}$ from EQ 5.22 - 5.23 as input for a Runge-Kutta numerical calculation with step size, h, equal to $10^{-4}$ minutes.

After setting the basal activity, $\mathrm{R}_{\mathrm{a}}=0.05, \mathrm{k}_{\alpha}$ and $\mathrm{k}_{-\alpha}$ were adjusted until Meth produced a maximum total TAAR activity peak intensity of 0.2 and a profile similar to the oocytes response to $10 \mu \mathrm{M}$ Meth, FIGURE 5.5A. Once these values for $\mathrm{k}_{\alpha}$ and $\mathrm{k}_{-\alpha}$ were found they were used for all the remaining Model $\mathrm{C}$ simulations. An interesting result is that in order to get the peak profile to match that in the biological data, $\mathrm{k}_{\alpha}<\mathrm{k}_{-\alpha}$ and $\mathrm{k}_{\alpha} \approx \mathrm{k}_{-\alpha}$ must be true. This makes sense from a biologi- 
Model C: Modeling the oocyte data

cal perspective as receptors controlling neural activity must respond quickly to signals then reset, ready for the next signal.

Next came adjusting the values for $\mathrm{k}_{\beta}$ and $\mathrm{k}_{-\beta}$. In FIGURE 5.5B - C, $\mathrm{k}_{\boldsymbol{\beta}}$ was chosen to give strong TAAR 1 inhibition, $\mathrm{k}_{\beta} / \mathrm{k}_{\alpha}=21.9$, and $\mathrm{k}_{-\beta}$ chosen to producing a rapid disassociation of the inhibitor, $\mathrm{k}_{-\beta} / \mathrm{k}_{-\alpha}=1.37$. The $\mathrm{k}_{\beta} / \mathrm{k}_{\alpha}$ and $\mathrm{k}_{-\beta} / \mathrm{k}_{-\alpha}$ ratios determine the shape of the response curves when both Meth and inhibitor are applied. The ratios in FIGURE 5.5C yield weak initial sub-basal response followed by a weak stimulation. Essentially nearly all TAAR1 was inhibited but due to the inhibitor's fast off-rate enough Meth-TAAR1 complex remained to give some stimulatory effect before it too disassociated. If one were to drop $\mathrm{k}_{\beta} / \mathrm{k}_{\alpha}$ much lower than $\sim 20$ while holding $\mathrm{k}_{-\beta} / \mathrm{k}_{-\alpha}$ constant, one would only see a stimulatory response (a peak). 


\section{Model C: Modeling the oocyte data}

FIGURE 5.5: Oocyte response to various stimuli: A) Meth only, B) Inhibitor only with a fast off-rate, $\mathrm{k}_{-\beta}, \mathrm{C}$ ) a combination of both Meth and Inhibitor. All the response curves been have normalized to their respective basal activities. The gap seen in C for the Total Active TAAR1 is a graphing artifact.
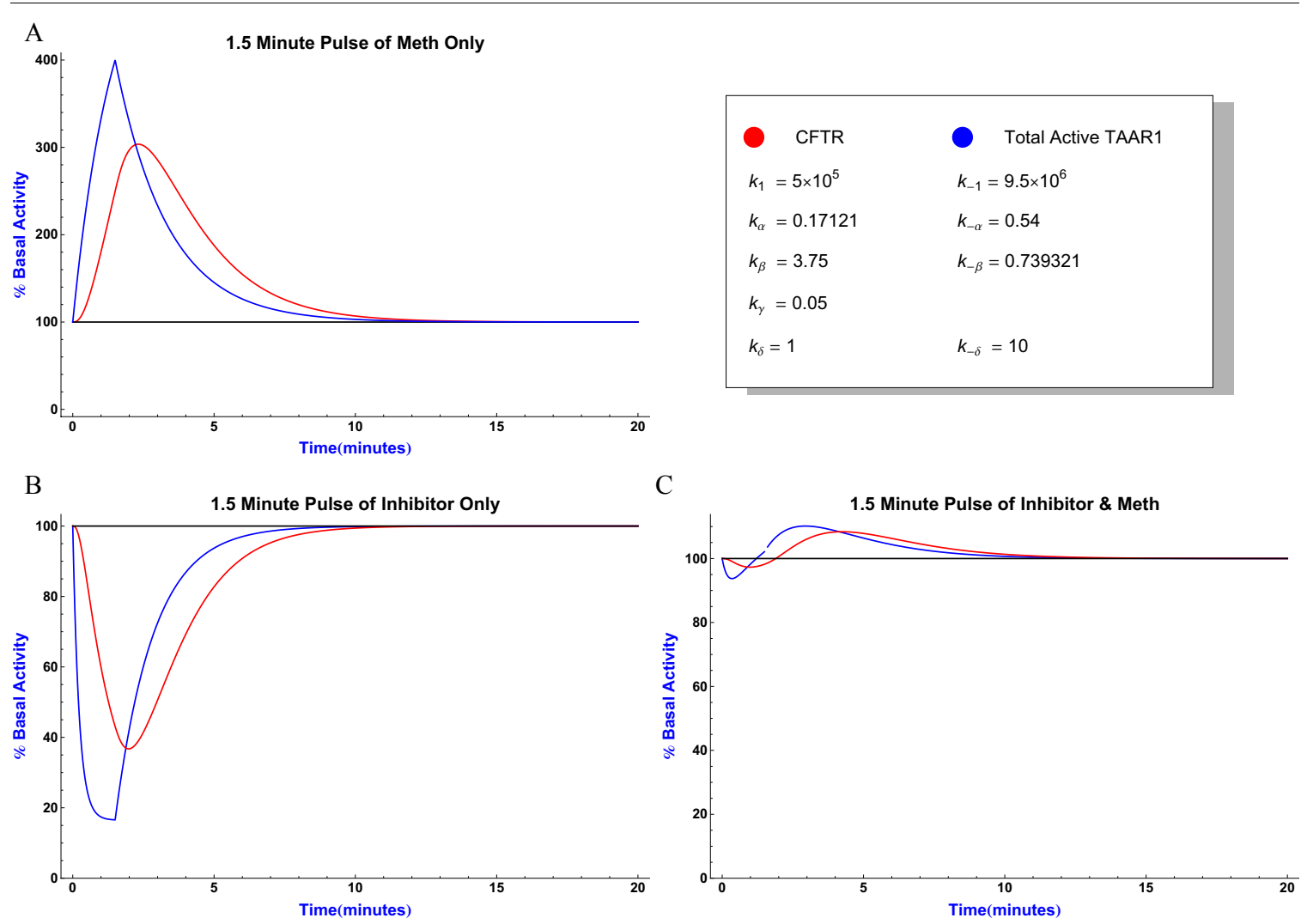

In moving to FIGURE 5.6, the only change made was to slow the inhibitor offrate $\left(\mathrm{k}_{\beta} / \mathrm{k}_{\alpha}=21.9\right)$. With $\mathrm{k}_{-\beta} / \mathrm{k}_{-\alpha}=0.60$, the inhibitor completely suppressed Meth stimulation. At this ratio, the response curve recovers to the basal level at about the same rate as seen in the biological data when the Meth response is completely suppressed. Increasing $\mathrm{k}_{\beta} / \mathrm{k}_{\alpha}=29.2$, FIGURE 5.7, just deepens the trough. However, decreasing $\mathrm{k}_{\beta} / \mathrm{k}_{\alpha}=11.68$, FIGURE 5.8, results in an initial Meth stimulation followed by a shallow sub-basal tough; not enough TAAR1 was inhibited. If $\mathrm{k}_{\beta} / \mathrm{k}_{\alpha}$ was reduced below $\sim 10$, then the sub-basal trough disappeared. 
Model C: Modeling the oocyte data

In the biological data there was little evidence that the $1 \mu \mathrm{M}$ test compounds inhibited $10 \mu \mathrm{M}$ Meth stimulation of TAAR1 and this model starts to reveal the reason. Since

$$
\frac{\mathrm{k}_{\beta}[\operatorname{Inh}]}{\mathrm{k}_{\alpha}[\text { Meth }]} \approx 10
$$

appears to be the lower limit at which a trough forms, except for possibly G5$\mathbf{1 1 4 s 5}$, the upper limit on $\mathrm{k}_{\beta} / \mathrm{k}_{\alpha}<100$ or the $1 \mu \mathrm{M}$ test compounds would produced a trough. Furthermore, limiting the estimate of $\mathrm{k}_{\beta} / \mathrm{k}_{\alpha}<30$ is reasonable or the 1 $\mu \mathrm{M}$ test compounds would produced obvious inhibition of the Meth stimulated peak. Since all $10 \mu \mathrm{M}$ test compounds produced a sub-basal trough, a estimate on the lower limit of $\mathrm{k}_{\beta} / \mathrm{k}_{\alpha}>15$ is also reasonable. 


\section{Model C: Modeling the oocyte data}

FIGURE 5.6: Slowing the inhibitor off-rate results in a total sub-basal response when both Meth and inhibitor are applied.
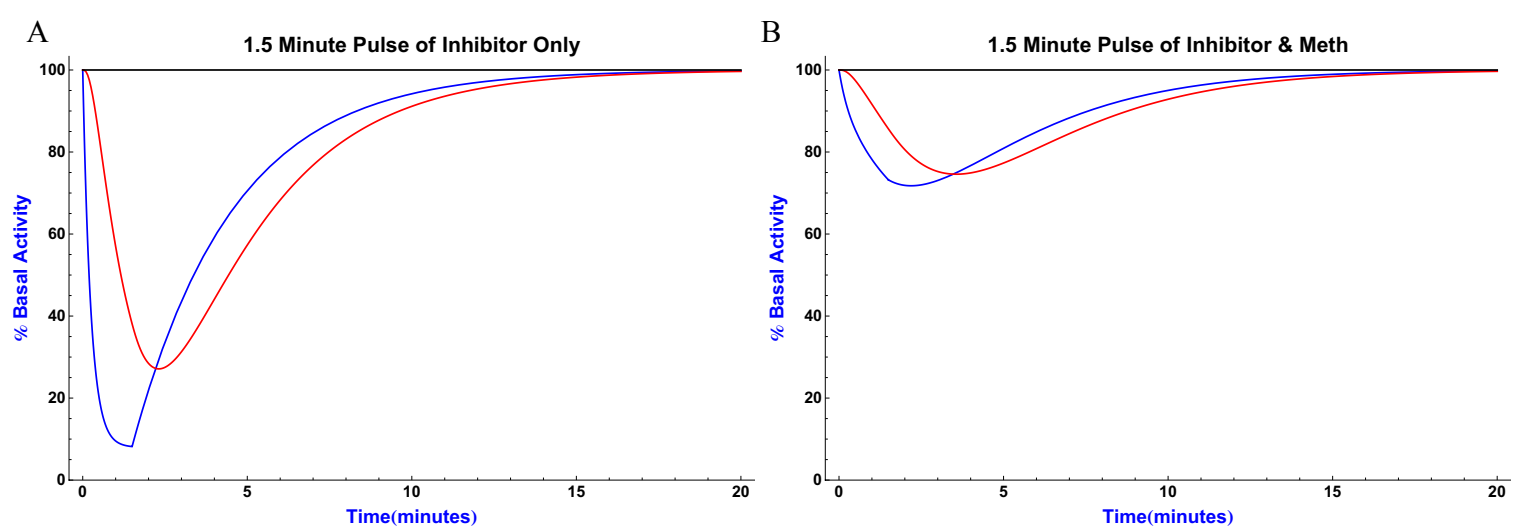

$\begin{array}{ll}\quad \text { CFTR } & \\ k_{1}=5 \times 10^{5} & k_{-1}=9.5 \times 10^{6} \\ k_{\alpha}=0.17121 & k_{-\alpha}=0.54 \\ k_{\beta}=3.75 & k_{-\beta}=0.325 \\ k_{\gamma}=0.05 & \\ k_{\delta}=1 & k_{-\delta}=10\end{array}$

FIGURE 5.7: Increasing the inhibitor on-rate results just deepens the trough when both Meth and inhibitor are applied.
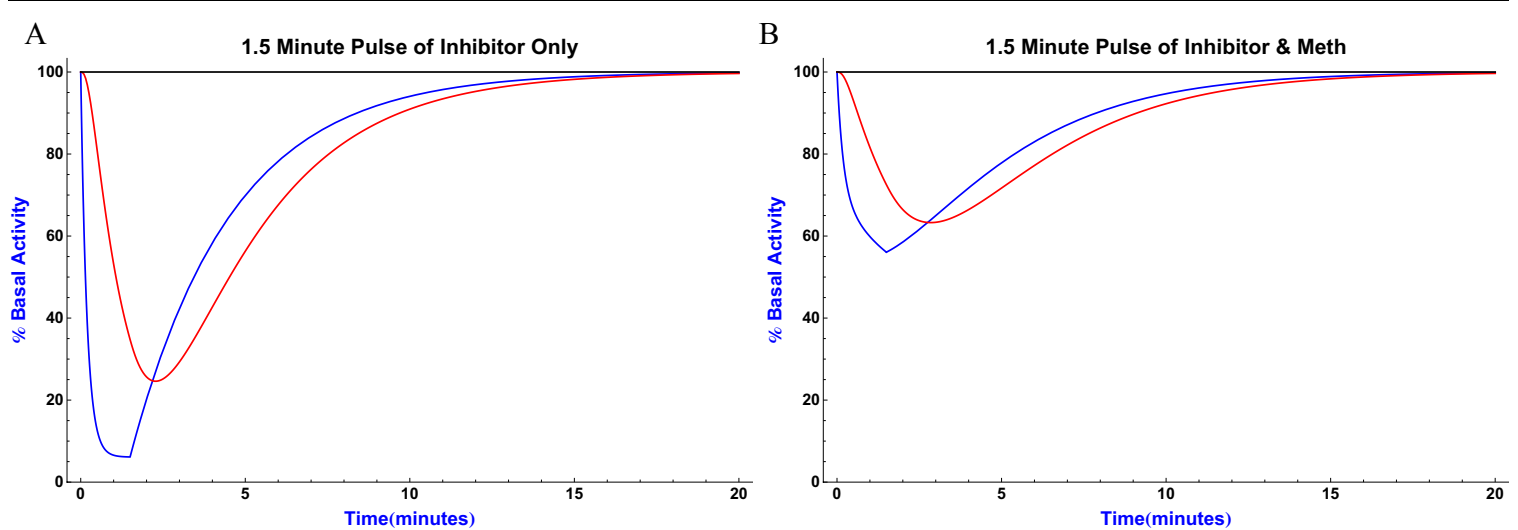

$\begin{array}{ll}\quad \text { CFTR } & \\ k_{1}=5 \times 10^{5} & k_{-1}=9.5 \times 10^{6} \\ k_{\alpha}=0.17121 & k_{-\alpha}=0.54 \\ k_{\beta}=5 & k_{-\beta}=0.325 \\ k_{\gamma}=0.05 & \\ k_{\delta}=1 & k_{-\delta}=10\end{array}$




\section{Stop Flow Model; a hybrid approach}

FIGURE 5.8: Decreasing the inhibitor on-rate too much prevents the complete inhibition of Meth stimulation when both Meth and inhibitor are applied.
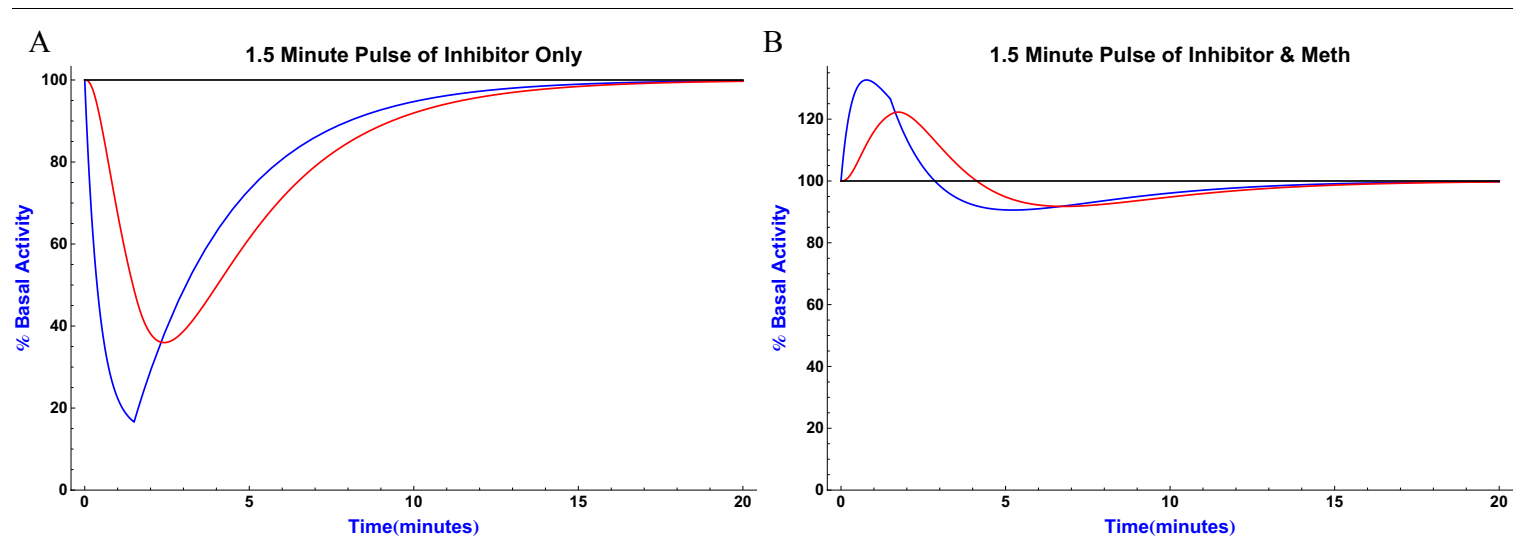

\begin{tabular}{|ll|}
\hline$\quad$ CFTR & \multicolumn{1}{c|}{ Total Active TAAR1 } \\
$k_{1}=5 \times 10^{5}$ & $k_{-1}=9.5 \times 10^{6}$ \\
$k_{\alpha}=0.17121$ & $k_{-\alpha}=0.54$ \\
$k_{\beta}=2$ & $k_{-\beta}=0.325$ \\
$k_{\gamma}=0.05$ & \\
$k_{\delta}=1$ & $k_{-\delta}=10$ \\
\hline
\end{tabular}

\subsection{Stop Flow Model; a hybrid approach}

The only response curve profile that proved elusive using Model C was the trough-peak-trough pattern. Considering how basic Model C was, it is not surprising that Model $\mathrm{C}$ can not produce the full range of data observed. After pondering the situation for awhile, it was realized that a hybrid model combing the TAAR1 kinetics of Model B, EQ 5. 8 - 5.13, and cAMP/CFTR kinetics of Model C, EQ 5.24 - 5.25, would work to produce this last profile, if the oocyte was immersed in a closed system for the duration of the compound application and then washed for the remaining duration of the simulation. This is not an accurate model for the experiments performed, but by having a more limited supply of Meth and inhibitor than the actual experiments, it serves as a conservative model. The closed system 


\section{Stop Flow Model; a hybrid approach}

makes the equilibrium equations valid. Once washing begins the Meth and Inhibitor concentrations are set to zero, and at that point the TAAR1 kinetics have an exact solution.

In order to produce the trough-peak-trough pattern seen in the response curves of G5-110s8, G5-112s5 and G5-114s5, the inhibitor must rapidly bind to $R_{a}, k_{5} / k_{3}$ $>\sim 60$, with a slower binding to $\mathrm{R}_{\mathrm{i}}, \mathrm{k}_{4} / \mathrm{k}_{3} \approx 15$. If $\mathrm{k}_{4} / \mathrm{k}_{3}$ is much above 15 , the Meth response will be completely inhibited. The other requirement for this response profile is that the equilibrium between $\mathrm{R}_{\mathrm{i}}$ and $\mathrm{R}_{\mathrm{a}}$ must be slow, $\mathrm{k}_{1} / \mathrm{k}_{3}<\sim 0.06$. The first trough in FIGURE 5.9B can be deepened by either increasing $\mathrm{k}_{5} / \mathrm{k}_{3}$ (not vary effective) or increasing $\mathrm{k}_{4} / \mathrm{k}_{3}$, which also reduces the size of the peak. 


\section{Concluding remarks}

FIGURE 5.9: By combining the TAAR1 components from Model B with the cAMP/CFTR components from Model $\mathrm{C}$ and using a stop-flow paradigm it is possible to produce the trough-peak-trough response profile seen from G5-110s8, G5-112s5 and G5-114s5.
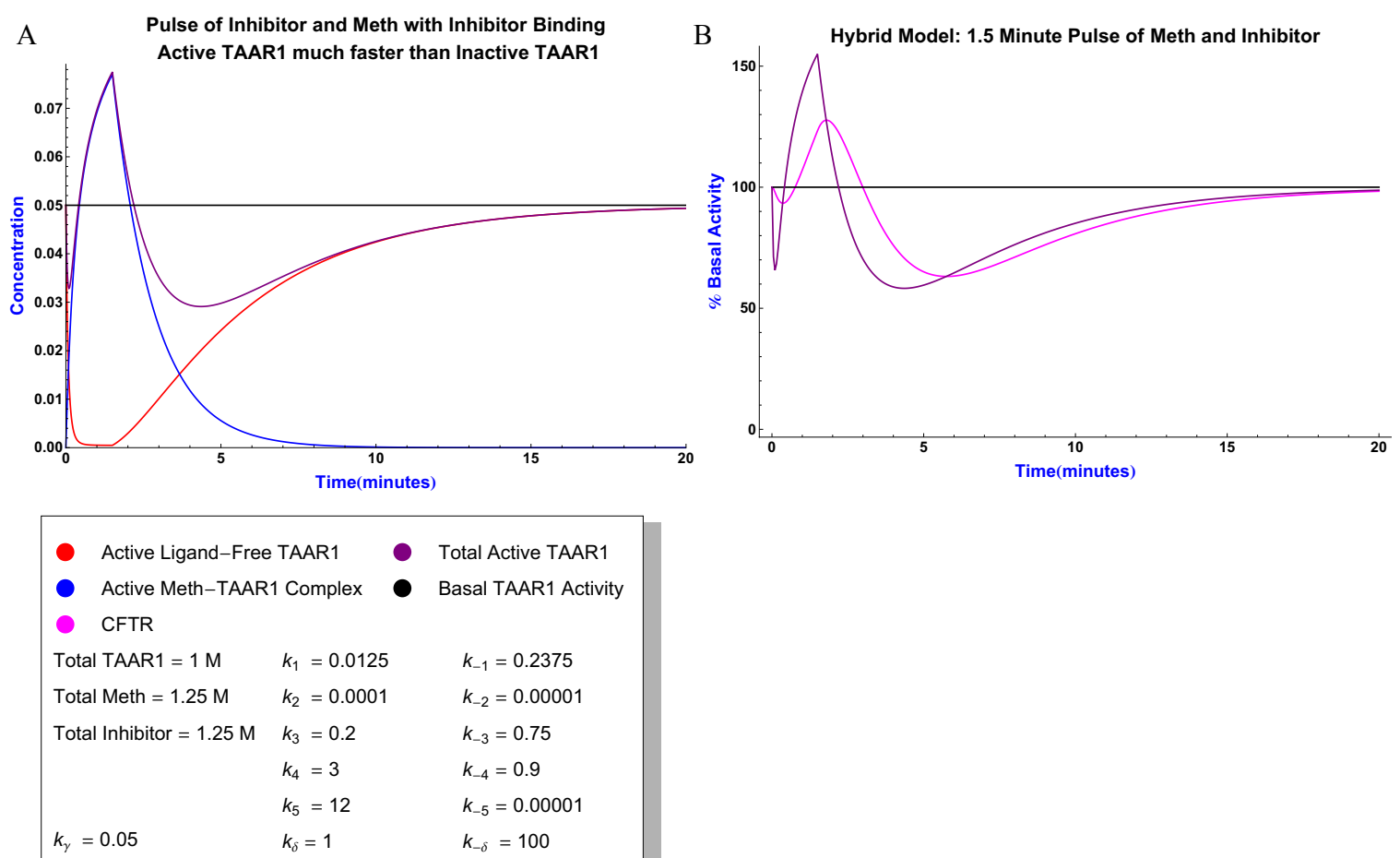

\subsection{Concluding remarks}

By modeling TAAR1 as a basally active receptor it was possible to qualitatively reproduce all the oocyte response curve profiles produced by the inhibitors tested. If TAAR1 was not basally active, it would not have been possible for an inhibitor to sub-basally suppress activity. Since a trough-peak-trough profile was observed with some of the tested inhibitors, the hybrid model suggests that the conversion rate of inactive to active TAAR 1 is less than $6 \%$ of the binding rate of Meth to inactive-TAAR1. The models also suggest that the tested inhibitors are binding inactive TAAR 1 at rates between 10 and 100 times faster than Meth binds. 


\section{Concluding remarks}

The models do not suggest upper limits on the inhibitor binding rate to active

TAAR1.

It is possible to fit the oocyte data with these models, but would require a special non-linear regression program to be written. Writing such a program can be done but it would not be a trivial task. Also, the resulting program would be slow. The simulations presented here took up to 20 minutes to run, although with optimization the code could run much faster. To find the best fit with a non-linear regression, one might have to run up to a thousand simulations. So even at 1.5 minutes per simulation, it could take a day to fit this type of data. A different experimental design, perhaps a steady-state one, could produce data that was easier to fit and use commercial software to do so. 
As more research is done on TAAR1, it is becoming increasingly clear that it is an important player in many neurological disorders, including addiction. The approval of any pharmaceutical targeting TAAR1 is years away, with the closest candidates (from Hoffmann-LaRoche) just entering phase 1 clinical trials. Since TAAR1 is activated by Meth and inhibiting it blocks Meth signaling, there is reason to hope that a pharmaceutical will be developed to help in the treatment of Meth addiction.

During an assay of G5-109s8 with an oocyte injected only with CFTR encoding RNA, G5-109s8 caused a drop in transmembrane conductance. Since TAAR1 was not present in the oocyte $(10 \mu \mathrm{M}$ Meth produced an $\sim 1 \mu \mathrm{S}$ drop in transmembrane conductance during this assay instead of the normal increase in conductance when TAAR1 is present), G5-109s8 produced an electrophysiological off-target effect. In order to further develop the thyronamine family of analogs the cause of the electrophysiological off-target effect observed in the oocyte, if confirmed by replicate experiments, will have to be identified. This off-target effect is particularly troubling as it may indicate potential adverse central nervous system, cardiovascular or mucus membrane (ulcers, diarrhea, cystic fibrosis, etc.) events in vivo. At a minimum, the identification project will require using G5-109s8 in a selectiv- 
ity and toxicity screen against ion channels, neurotransmitter receptors and transporters, hERG, and the superfamily of cytochrome P450 (CYP P450). A good list to start from would be the CEREP panel used by Revel, et al., consisting of 149 target proteins, transporters, ion channels, and receptors. ${ }^{9}$ The afore mentioned selectivity and toxicology screen can serve as a further test for any potential offtarget effects. Furthermore, as part of due diligence in a medicinal chemistry project, a screen needs to be done.

Since the ultimate goal is to develop a TAAR1 ligand for the treatment of Meth addiction and not to develop a member of the thyronamine family into such a pharmaceutical, one could start a new hit generation program under the assumption that a random hit will have a different selectivity profile. As was done here, a TEVC oocyte control experiment can be used to detect electrophysiological off-target effects associated with the new TAAR1 hits. Under this approach, one would keep developing new hits until one was found that did not have electrophysiological offtarget effects. Another option is to bust Hoffmann-LaRoche's TAAR1 patents (i.e.: develop analogs of their compounds that exploit loopholes in their patents).

Although the oocyte assays were performed only once as first pass activity screens, more evidence was found that TAAR1 has basal activity (constitutively active, for those who prefer that term) and 6 new TAAR1 inhibitors (inverse agonists) were developed. Also, although 32a-c (FIGURE 6.9) would have to be re- 
synthesized, they would also be interesting compounds to assay as they would explore the SAR necessity of the terminal amine found in ET-92. By testing the compounds in oocytes injected with hTAAR1 and hCFTR encoding RNA and qualitatively comparing the response curves to the results of kinetic simulations based on three models, estimates of several kinetic parameters were found relative to the binding rate of Meth to inactive TAAR1. By approximating the basal activity of TAAR 1 to be $5 \%$ of the total ligand-free TAAR 1 , the conversion rate of inactive TAAR 1 to active TAAR 1 is less than $6 \%$ of the binding rate of Meth to inactive TAAR1. It is also estimated that the inhibitors bind to inactive TAAR1 between 10 and 100 times faster than Meth. Based on this work there is no upper rate limit for the inhibitors binding to active TAAR1. 


\subsection{Synthetic Methods}

\subsubsection{Equipment and Chemicals}

NMR spectroscopy was performed on either a Bruker Avance II+ $400 \mathrm{MHz}$ NMR Spectrometer or a Bruker Avance III 600 MHz NMR Spectrometer, both equipped with Bruker 5mm BBO probes, running Topspin software.

HPLC performed on Agilent (Varian) prep HPLC with $25 \mathrm{~mL}$ pumpheads, running Galaxy Star software. Flash chromatography used a Teledyne Isco CombiFlash Rf-200 psi, model 68-5230-008.

Chemicals were bought from Sigma-Aldrich, TCIAmerica, Fisher Scientific or EMD and used as is.

\subsubsection{Compounds}

IUPAC compound names were generated by ChemDraw Ultra 11.0.

GX-YsZ refers to laboratory notebook, first page number of the procedure and step number of the final product. The $\mathrm{G}$ is shorthand Grandy, $\mathbf{X}$ is $1-5, \mathbf{Y}$ is 001 150 and $\mathbf{Z}$ is $1-50$. For example, G4-120s 5 is the $5^{\text {th }}$ step of the procedure beginning on page 120 in the laboratory notebook Grandy4. 


\section{Synthetic Methods}

\subsubsection{5-bromo-2-(hexyloxy)benzaldehyde: G4-12s16}

5-bromo-2-hydroxybenzaldehyde (26.69 g, $132.77 \mathrm{mmol}), \mathrm{K}_{2} \mathrm{CO}_{3}(20.179 \mathrm{~g}$,

$146 \mathrm{mmol})$ and anhydrous dimethylformamide $(200 \mathrm{~mL})$ were combined in a round bottom flask. While stirring, 1-bromohexane (24.108 g, $20.5 \mathrm{~mL}, 146$

mmol) was added, then the reaction was stirred for 18 hours. The reaction was worked-up by adding water $(500 \mathrm{~mL})$, then extracting with hexanes $(4 \times 250 \mathrm{~mL})$. The combined hexane phases were extracted with $0.05 \mathrm{M} \mathrm{NaOH}(2 \times 500 \mathrm{~mL})$, then dried with $\mathrm{MgSO}_{4}$. After filtering off the $\mathrm{MgSO}_{4}$, the filtrate was concentrated via rotary evaporation to a yellow oil (36.8359 $\mathrm{g}, 97.3 \%$ yield).

Optional: The oil was then distilled under vacuum (46 mTorr, bp 104-130 ${ }^{\circ} \mathrm{C}$ ) using an unjacketed shortpath distillation head with Vigreux indentations. Colorless oil (31.2881 g, 82.6\% yield).

Note: The product tends to exist as a supercooled liquid but under just the right conditions, it will crystallize $\left(\mathrm{mp}>23^{\circ} \mathrm{C}\right.$ ) however it will melt if disturbed (for example by the applied pressure of a spatula attempting to remove the solid). Also, as the product ages it tends to slowly turn yellow.

${ }^{1} \mathrm{H}-\mathrm{NMR} \delta(\mathrm{ppm})\left(\right.$ DMSO-d $\left._{6}\right): 10.286(\mathrm{~s}, 1 \mathrm{H}), 7.790(\mathrm{dd}, \mathrm{J}=9.0 \mathrm{~Hz}, 3.0 \mathrm{~Hz}$, 1H), $7.724(\mathrm{~d}, \mathrm{~J}=3.0 \mathrm{~Hz}, 1 \mathrm{H}), 7.225(\mathrm{~d}, \mathrm{~J}=9.0 \mathrm{~Hz}, 1 \mathrm{H}), 4.126(\mathrm{t}, \mathrm{J}=6.6 \mathrm{~Hz}, 2 \mathrm{H})$, $1.73-1.79(\mathrm{~m}, 2 \mathrm{H}), 1.41-1.46(\mathrm{~m}, 2 \mathrm{H}), 1.26-1.34(\mathrm{~m}, 4 \mathrm{H}), 0.870(\mathrm{t}, \mathrm{J}=6.9 \mathrm{~Hz}, 3 \mathrm{H})$. 


\section{Synthetic Methods}

${ }^{13}$ C-NMR $\delta(p p m)\left(\right.$ DMSO-d $\left._{6}\right): 187.99,160.08,138.45,129.69,125.7,116.33$,

$112.23,68.82,30.87,28.28,25.02,21.98,13.84$.

TABLE 7.1: High resolution mass spectrum (ESI) of 5-bromo-2-(hexyloxy)benzaldehyde (G4-012s16)

\begin{tabular}{|c|c|c|c|}
\hline Species & Chemical Formula & Calculated $m / z$ & Observed $m / z$ \\
\hline$[\mathbf{M}+\mathbf{H}]^{+}$ & $\mathrm{C}_{13} \mathrm{H}_{18} \mathrm{BrO}_{2}^{+}$ & 285.04847 & 285.04869 \\
\hline$[\mathbf{M}+\mathbf{N a}]^{+}$ & $\mathrm{C}_{13} \mathrm{H}_{17} \mathrm{BrNaO}_{2}^{+}$ & 307.03041 & 307.03115 \\
\hline$\left[\mathbf{M}+\mathrm{CH}_{3}\right]^{+}$ & $\mathrm{C}_{14} \mathrm{H}_{20} \mathrm{BrO}_{2}^{+}$ & 299.06412 & 299.06453 \\
\hline$\left[\mathrm{M}+\mathrm{CH}_{3}+\mathrm{H}+\mathrm{Na}\right]^{+}$ & $\mathrm{C}_{14} \mathrm{H}_{21} \mathrm{BrNaO}_{2}^{+}$ & 323.06171 & 323.02624 \\
\hline$[\mathrm{M}+\mathrm{MeOH}+\mathrm{Na}]^{+\mathbf{a}}$ & $\mathrm{C}_{14} \mathrm{H}_{21} \mathrm{BrNaO}_{3}^{+}$ & 339.05663 & 339.05742 \\
\hline
\end{tabular}

a. This is the sodium cation form of the hemiacetal with $\mathrm{MeOH}$.

\subsubsection{Hexyl 2-(hexyloxy)-5-nitrobenzoate: G5-047s10}

2-hydroxy-5-nitrobenzoic acid (30 mmol, 5.4963 g), $\mathrm{K}_{2} \mathrm{CO}_{3}(12.4389 \mathrm{~g}, 90$

mmol) and anhydrous dimethylformamide $(50 \mathrm{~mL})$ were combined in a round bottom flask. While stirring, 1-bromohexane (14.8563 g, $12.63 \mathrm{~mL}, 90 \mathrm{mmol})$ was added, then the reaction was stirred for 25 hours at $80^{\circ} \mathrm{C}$. The reaction was worked-up by adding water $(250 \mathrm{~mL})$, then adjusting the $\mathrm{pH}=2$ with $12 \mathrm{M} \mathrm{HCl}(\sim$ $6 \mathrm{~mL})$. The mixture was extracted with hexanes $(2 \times 100 \mathrm{~mL})$. The combined hexane phases were washed with water $(2 \times 100 \mathrm{~mL})$, and dried with $\mathrm{MgSO}_{4}$. After filtering off the $\mathrm{MgSO}_{4}$, the solution was concentrated by rotatory evaporation (10.23 g, 97.1\% yield).

${ }^{1} \mathrm{H}-\mathrm{NMR} \delta(\mathrm{ppm})\left(\mathrm{DMSO}_{\mathrm{d}}\right): 8.45(1 \mathrm{H}, \mathrm{d}, \mathrm{J}=2.95 \mathrm{~Hz}), 8.39(1 \mathrm{H}, \mathrm{dd}, \mathrm{J}=$ 9.24, $2.98 \mathrm{~Hz}), 7.38(1 \mathrm{H}, \mathrm{d}, \mathrm{J}=9.29 \mathrm{~Hz}), 4.27(2 \mathrm{H}, \mathrm{t}, \mathrm{J}=6.54 \mathrm{~Hz}), 4.20(2 \mathrm{H}, \mathrm{t}, \mathrm{J}$ 


\section{Synthetic Methods}

$=6.28 \mathrm{~Hz}), 1.64-1.80(4 \mathrm{H}, \mathrm{m}), 1.35-1.50(4 \mathrm{H}, \mathrm{m}), 1.25-1.35(6 \mathrm{H}, \mathrm{m}), 0.84-0.92$

$(6 \mathrm{H}, \mathrm{m})$.

\subsubsection{3 (2-(hexyloxy)-5-nitrophenyl)(pyrrolidin-1-yl)methanone: G5-090s11}

(2-hydroxy-5-nitrophenyl)(pyrrolidin-1-yl)methanone (G5-088s8, 0.897 g, 2.8

$\mathrm{mmol}), \mathrm{K}_{2} \mathrm{CO}_{3}(0.580 \mathrm{~g}, 4.2 \mathrm{mmol})$ and anhydrous dimethylformamide $(5 \mathrm{~mL})$ were combined in a round bottom flask. While stirring, 1-bromohexane (0.693 g, $0.590 \mathrm{~mL}, 4.2 \mathrm{mmol}$ ) was added, then the reaction was stirred for 10 hours at 80 ${ }^{\circ} \mathrm{C}$. The reaction was worked-up by adding $2 \mathrm{M} \mathrm{HCl}(6 \mathrm{~mL})$, then extracting with hexanes $(3 \times 5 \mathrm{~mL})$. The combined hexane phases were washed with water $(2 \times 5$ $\mathrm{mL}$ ), then filtered through a fine sintered glass funnel, and the filtrate dried with $\mathrm{MgSO}_{4}$. After filtering off the $\mathrm{MgSO}_{4}$, the solution was concentrated by rotatory evaporation to an oil.

${ }^{1} \mathrm{H}-\mathrm{NMR} \delta(\mathrm{ppm})\left(\mathrm{DMSO}_{-} \mathrm{d}_{6}\right): 8.28(1 \mathrm{H}, \mathrm{dd}, \mathrm{J}=9.19,2.90 \mathrm{~Hz}), 8.06(1 \mathrm{H}, \mathrm{d}, \mathrm{J}$ $=2.89 \mathrm{~Hz}), 7.31(1 \mathrm{H}, \mathrm{d}, \mathrm{J}=9.24 \mathrm{~Hz}), \# \# \# 5.76(0 \mathrm{H}, \mathrm{s}), 4.18(2 \mathrm{H}, \mathrm{t}, \mathrm{J}=6.27 \mathrm{~Hz})$, $3.46(2 \mathrm{H}, \mathrm{t}, \mathrm{J}=6.55 \mathrm{~Hz}), 3.13(2 \mathrm{H}, \mathrm{t}, \mathrm{J}=6.55 \mathrm{~Hz}), 1.76-1.92(4 \mathrm{H}, \mathrm{m}), 1.67-1.76$ (2 H, m), 1.35-1.45 (2 H, m), 1.26-1.35 (4 H, m), 0.88 (3 H, t, J = 6.83 Hz).

${ }^{13} \mathrm{C}-\mathrm{NMR} \delta(\mathrm{ppm})\left(\mathrm{DMSO}_{6}\right): 163.9,159.4,140.4,128.1,126.3,123.3$, $112.8,69.0,46.9,45.3,30.8,28.2,25.4,24.9,24.1,22.0,13.8$. 


\section{Synthetic Methods}

\subsubsection{2-(hexyloxy)-5-nitrobenzaldehyde: G5-120s11}

2-hydroxy-5-nitrobenzaldehyde (30 mmol, $5.0136 \mathrm{~g}), \mathrm{K}_{2} \mathrm{CO}_{3}(60 \mathrm{mmol}$,

$8.2926 \mathrm{~g})$ and anhydrous dimethylformamide $(100 \mathrm{~mL})$ were combined in a round bottom flask. While stirring, 1-bromohexane (60 mmol, $9.9042 \mathrm{~g}, 8.422 \mathrm{~mL}$ ) was added, then the reaction was stirred for 18 hours at $80^{\circ} \mathrm{C}$. The reaction was worked-up by adding water $(250 \mathrm{~mL})$, then extracting with ethyl acetate $(50 \mathrm{~mL})$. The aqueous phase was extracted with ethyl acetate (EtOAc, $3 \times 100 \mathrm{~mL})$. The ethyl acetate phases were combined then extracted with $0.6 \mathrm{M} \mathrm{HCl}(100 \mathrm{~mL})$, then brine $(50 \mathrm{~mL})$. The organic phase was dried with $\mathrm{MgSO}_{4}$. After filtering off the $\mathrm{MgSO}_{4}$, the solution was concentrated by rotatory evaporation to an oil. The oil was purified by plug filtration through silica in a $150 \mathrm{~mL}$ sintered glass funnel, eluting with hexanes $(150 \mathrm{~mL})$ followed by dichloromethane (DCM, $500 \mathrm{~mL})$, and collecting the hexanes and DCM eluants as separate fractions. The DCM fraction was concentrated by rotatory evaporation to a tan solid. The solid was dissolved in hexanes $(100 \mathrm{~mL})$ and DCM $(10 \mathrm{~mL})$, and the resulting solution was concentrated by rotatory evaporation at $40{ }^{\circ} \mathrm{C}$ until the DCM was removed. As the mixture cooled, crystals formed, which were filtered off using a medium sinter glass funnel and washed with hexanes ( $8.00 \mathrm{~g}, 53 \%$ yield $)$.

\footnotetext{
${ }^{1} \mathrm{H}-\mathrm{NMR} \delta(\mathrm{ppm})\left(\mathrm{DMSO}_{6}\right): 10.35(1 \mathrm{H}, \mathrm{s}), 8.49(1 \mathrm{H}, \mathrm{dd}, \mathrm{J}=9.23,2.99 \mathrm{~Hz})$, $8.42(1 \mathrm{H}, \mathrm{d}, \mathrm{J}=2.97 \mathrm{~Hz}), 7.48(1 \mathrm{H}, \mathrm{d}, \mathrm{J}=9.27 \mathrm{~Hz}), 4.30(2 \mathrm{H}, \mathrm{t}, \mathrm{J}=6.43 \mathrm{~Hz})$,
} 


\section{Synthetic Methods}

1.78-1.87 (2 H, m), 1.42-1.52 (2 H, m), 1.30-1.36 (4 H, m), 0.89 (3 H, t, J = 6.84

$\mathrm{Hz})$.

${ }^{13} \mathrm{C}-\mathrm{NMR} \delta(\mathrm{ppm})\left(\mathrm{DMSO}_{6}\right): 187.9,165.0,140.6,130.9,123.9,123.5$, $114.7,69.8,30.8,28.2,24.9,22.0,13.8$.

\subsubsection{5 (5-bromo-2-(hexyloxy)phenyl)(phenyl)methanol: G4-19s13}

In a $20 \mathrm{~mL}$ vial with a septum cap, 5-bromo-2-(hexyloxy)benzaldehyde (G4-

012s16, $0.5 \mathrm{~g}, 1.75 \mathrm{mmol}$ ) was dissolved in anhydrous tetrahydrofuran (THF, 10

$\mathrm{mL}$ ), and the resulting solution cooled to $0{ }^{\circ} \mathrm{C}$. To the solution, phenylmagnesiumbromide (1.0 M in THF, $2.6 \mathrm{~mL}, 2.6 \mathrm{mmol})$ was added dropwise, and the reaction was allowed to warm to room temperature over 4 hours. The reaction was acidified to $\mathrm{pH} 6$ with $0.1 \mathrm{M} \mathrm{HCl}(\sim 50 \mathrm{~mL})$, then extracted with ethyl acetate (EtOAc, $3 \mathrm{x}$ $20 \mathrm{~mL})$. The combined EtOAc phases were washed with water $(2 \times 25 \mathrm{~mL})$, dried over $\mathrm{MgSO}_{4}$. After filtration, silica gel $(\sim 1 \mathrm{~g})$ was added to the filtrate, then concentrated by rotatory evaporation to a powder. The powder was used as a dry-load for silica chromatography (FIGURE 7.1). Fractions $15-18$ were combined and concentrated by rotatory evaporation to a white solid $(0.4333 \mathrm{~g}, 68.2 \%$ yield $)$.

${ }^{1} \mathrm{H}-\mathrm{NMR} \delta(\mathrm{ppm})\left(\mathrm{DMSO}_{6}\right): 7.6384(\mathrm{~d}, \mathrm{~J}=2.5 \mathrm{~Hz}, 1 \mathrm{H}), 7.3478(\mathrm{dd}, \mathrm{J}=8.7$ Hz, $2.5 \mathrm{~Hz}, 1 \mathrm{H}), 7.24$ - 7.33 (m, 4H), 7.16 - 7.22 (m, 1H), 6.8906 (d, J = 8.7 Hz), 


\section{Synthetic Methods}

$5.902(\mathrm{~d}, \mathrm{~J}=4.4 \mathrm{~Hz}, 1 \mathrm{H}), 5.835(\mathrm{~d}, \mathrm{~J}=4.4 \mathrm{~Hz}, 1 \mathrm{H}), 3.87-3.94(\mathrm{~m}, 2 \mathrm{H}), 1.60-1.71$

(m, 2H), 1.30-1.38 (m, 2H), 1.23-1.30 (m, 4H), $0.874(\mathrm{t}, \mathrm{J}=6.76 \mathrm{~Hz}, 3 \mathrm{H})$.

TABLE 7.2: High resolution MS (ESI) of (5-bromo-2-(hexyloxy)(phenyl))(phenyl)methanol (G4-019s13)

\begin{tabular}{|c|c|c|c|}
\hline Species & Chemical Formula & Calculated $m / z$ & Observed $m / z$ \\
\hline$[\mathbf{M}-\mathbf{O H}]^{+}$ & $\mathrm{C}_{19} \mathrm{H}_{22} \mathrm{BrO}^{+}$ & 345.08485 & 345.08485 \\
\hline$\left[\mathrm{M}+\mathrm{Na}^{+}\right.$ & $\mathrm{C}_{19} \mathrm{H}_{23} \mathrm{BrNaO}_{2}^{+}$ & 345.08485 & 345.08485 \\
\hline
\end{tabular}




\section{Synthetic Methods}

FIGURE 7.1: Chromatographic purification of G4-019s9.

Sample: grandy4-017s9

RediSep Column: Silica 40g SN: E041039E8B947 Lot: 1920177010W

Flow Rate: $40 \mathrm{ml} / \mathrm{min}$

Equilibration Volume: 5.0 CV

Initial Waste: $0.0 \mathrm{CV}$

Air Purge: $1.0 \mathrm{~min}$

Solvent: A1 hexane

Solvent: B1 ethyl acetate
Rf 200 : Isco Demo

Thursday 09 February 2012 01:14PM

Peak Tube Volume: Max.

Non-Peak Tube Volume: Max.

Loading Type: Liquid

Wavelength 1 (red): 254nm

Peak Width: 2 min

Threshold: 0.05 AU

Wavelength 2 (purple): 280nm

Run Notes:

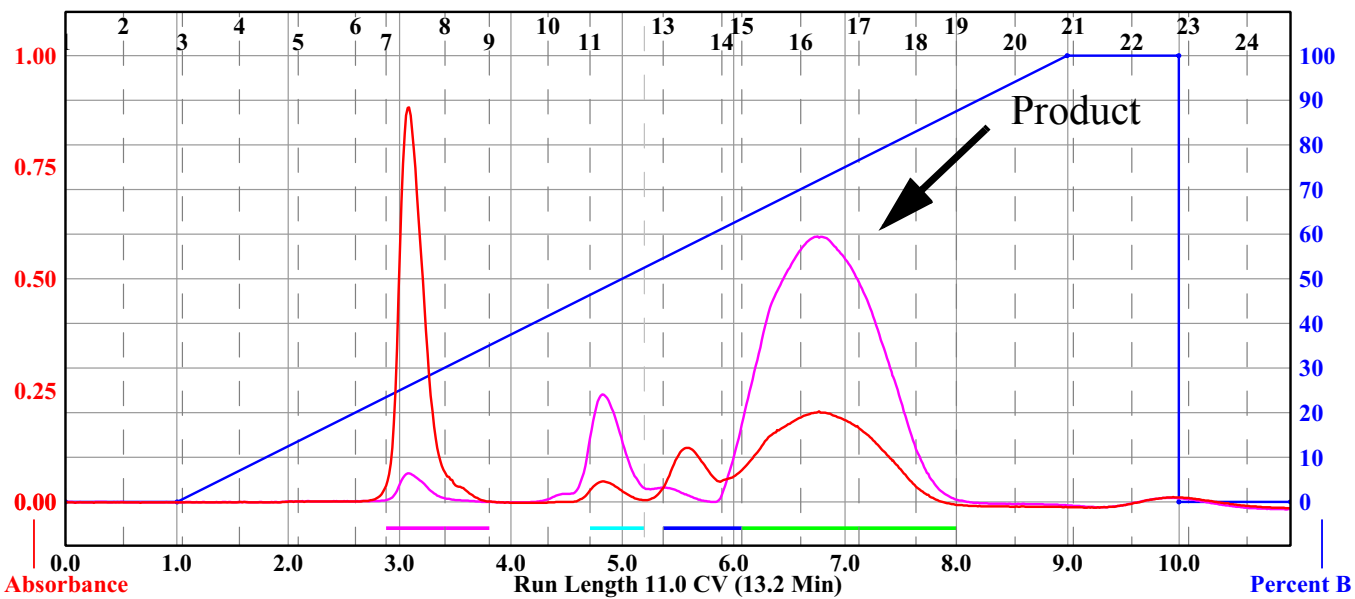

Rack A

Peak \#

Start Tube

\begin{tabular}{|c|c|c|c|c|}
\hline (70) & (69) & 68 & (67) & 66 \\
\hline (61) & 62 & 63 & (64) & (65) \\
\hline 60 & (59) & 58 & (57) & (56) \\
\hline (51) & (52) & 53 & (54) & (55) \\
\hline (50) & (49) & (48) & (47) & (46) \\
\hline (41) & (42) & (43) & (44) & (45) \\
\hline (40) & (39) & (38) & (37) & (36) \\
\hline (31) & (32) & (33) & (34) & (35) \\
\hline (30) & (29) & (28) & (27) & (26) \\
\hline (21) & (22) & (23) & (24) & (25) \\
\hline (20) & (19) & (18) & (17) & (16) \\
\hline (11) & (12) & & & (15) \\
\hline (10) & (9) & & & (6) \\
\hline (1) & (2) & (3) & (4) & (5) \\
\hline
\end{tabular}

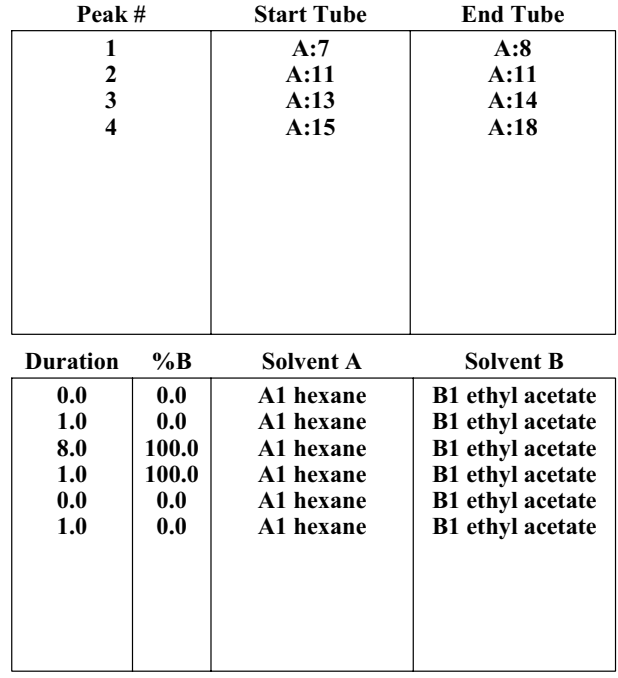

Page 1 of 1 


\section{Synthetic Methods}

\subsubsection{6 (5-(4-fluorophenoxy)-2-(hexyloxy)phenyl)(phenyl)methanol: G4-74s14}

Under an $\mathrm{N}_{2}$ atmosphere and in a round bottom flask, 5-(4-fluorophenoxy)-2-

(hexyloxy)benzaldehyde $(1.5150 \mathrm{~g}, 4.79 \mathrm{mmol})$ was dissolved in anhydrous tetrahydrofuran (THF, $50 \mathrm{~mL}$ ), then cooled to $0{ }^{\circ} \mathrm{C}$. Phenylmagnesiumbromide $(1.0 \mathrm{M}$ in THF, $9.5 \mathrm{~mL}, 9.5 \mathrm{mmol}, 2 \mathrm{eq})$ was added dropwise to the reaction. The reaction was allowed to warm to room temperature over at least 4 hours. The reaction was added to $2.4 \mathrm{M} \mathrm{HCl}(100 \mathrm{~mL})$, then extracted with ethyl acetate (EtOAc, 3 x 25 $\mathrm{mL})$. The combined EtOAc phases were washed with water $(2 \times 100 \mathrm{~mL})$, brine $(1$ x $25 \mathrm{~mL})$, and then dried over $\mathrm{MgSO}_{4}$. After filtration, silica gel ( $\left.\sim \mathrm{g}\right)$ was added to the filtrate, and then concentrated by rotatory evaporation to a powder. The powder was used as a dry-load for silica chromatography (see FIGURE 7.2). Fractions $24-40$ combined and concentrated by rotatory evaporation to a pale yellow oil (1.6727 g, 88.5\% yield).

${ }^{1} \mathrm{H}-\mathrm{NMR} \delta(\mathrm{ppm})\left(\mathrm{DMSO}_{6}\right): 7.24$ - $7.31(\mathrm{~m}, 4 \mathrm{H}), 7.228(\mathrm{~d}, \mathrm{~J}=3.0 \mathrm{~Hz}, 1 \mathrm{H})$, $7.16-7.22(\mathrm{~m}, 3 \mathrm{H}), 6.95-7.00(\mathrm{~m}, 2 \mathrm{H}), 6.922(\mathrm{~d}, \mathrm{~J}=9.0 \mathrm{~Hz}, 1 \mathrm{H}), 6.848(\mathrm{dd}, \mathrm{J}=$ $9.0 \mathrm{~Hz}, 3.0 \mathrm{~Hz}, 1 \mathrm{H}), 5.912(\mathrm{~d}, \mathrm{~J}=9.3 \mathrm{~Hz}, 1 \mathrm{H}), 5.730$ (d, J = 9.3 Hz, 1H), 3.85-3.93 $(\mathrm{m}, 2 \mathrm{H}), 1.62-1.70(\mathrm{~m}, 2 \mathrm{H}), 1.32-1.39(\mathrm{~m}, 2 \mathrm{H}), 1.26-1.32(\mathrm{~m}, 4 \mathrm{H}), 0.881(\mathrm{t}, \mathrm{J}=6.9$ $\mathrm{Hz}, 3 \mathrm{H})$.

${ }^{13} \mathrm{C}-\mathrm{NMR} \delta(\mathrm{ppm})\left(\mathrm{DMSO}_{\mathrm{d}}\right): 157.64(\mathrm{~d}, \mathrm{~J}=239.9 \mathrm{~Hz}), 153.94,151.09$, 149.73, 144.80, 135.38, 127.82, 126.65, 126.54, 119.20 (d, J = 7.5 Hz), 117.92, 


\section{Synthetic Methods}

$117.16,116.33$ (d, J = 22.6 Hz), 112.58, 68.22, 67.90, 30.93, 28.69, 25.22, 22.03, 13.8.

${ }^{19} \mathrm{~F}-\mathrm{NMR} \delta(\mathrm{ppm})\left(\mathrm{DMSO}-\mathrm{d}_{6}\right.$, with $\mathrm{C}_{6} \mathrm{~F}_{6}$ as reference at $\left.-164.9 \mathrm{ppm}\right):-123.37$ to -123.47 .

TABLE 7.3: High resolution MS (ESI) of 5-(4-fluorophenoxy)-2-(hexyloxy)(phenyl)methanol (G4-074s14)

\begin{tabular}{|c|c|c|c|}
\hline Species & Chemical Formula & Calculated $\boldsymbol{m} / \mathbf{z}$ & Observed $\boldsymbol{m} / \boldsymbol{z}$ \\
\hline$[\mathbf{M}+\mathbf{H}]^{+}$ & $\mathbf{C}_{\mathbf{1 9}} \mathbf{H}_{\mathbf{2 2}} \mathbf{F O}_{\mathbf{3}}{ }^{+}$ & 317.15475 & 371.16291 \\
\hline$[\mathbf{M}+\mathbf{N a}]^{+}$ & $\mathbf{C}_{\mathbf{1 9}} \mathbf{H}_{21} \mathbf{F N a O}_{\mathbf{3}}{ }^{+}$ & 339.13669 & 339.13728 \\
\hline$[\mathbf{M}+\mathbf{M e O H}+\mathbf{N a}]^{+\mathbf{a}}$ & $\mathbf{C}_{\mathbf{2 0}} \mathbf{H}_{\mathbf{2 5}} \mathbf{F N a O}_{\mathbf{4}}{ }^{+}$ & 371.16291 & 371.16289 \\
\hline
\end{tabular}

a. This is the sodium cation form of the hemiacetal with $\mathrm{MeOH}$. 


\section{Synthetic Methods}

FIGURE 7.2: Chromatographic purification of G4-074s12.

Sample: grandy4-074s 12

RediSep Column: Silica 40g SN: E04103BAEBBF Lot: $1922189010 X$ Flow Rate: $40 \mathrm{ml} / \mathrm{min}$

Equilibration Volume: 5.0 CV

Initial Waste: $0.0 \mathrm{CV}$

Air Purge: $1.0 \mathrm{~min}$

Solvent: A1 hexane

Solvent: B1 ethyl acetate
Rf 200 : OHSU COHEN RF200\#1

Thursday 12 April 2012 09:36AM

Peak Tube Volume: Max.

Non-Peak Tube Volume: Max.

Loading Type: Solid

Wavelength 1 (red): 254nm

Peak Width: 2 min

Threshold: 0.05 AU

Wavelength 2 (purple): $280 \mathrm{~nm}$

Run Notes:

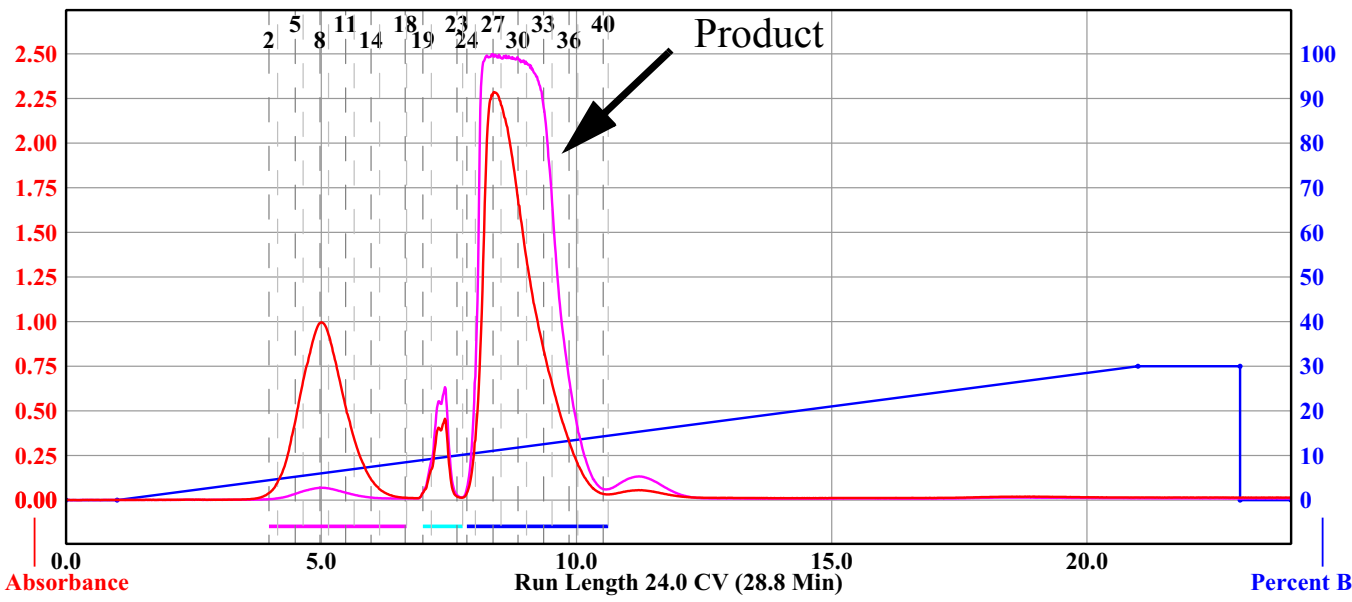

Rack A

\begin{tabular}{|c|c|c|c|c|c|}
\hline 108 & (107 & IOT & 105 & (104 & 103 \\
\hline (20) & $\mathscr{B}$ & $\mathscr{O P}$ & (10) & (1) & 102 \\
\hline ஏூ & פ5 & $\mathscr{H}$ & 93 & (2) & (21) \\
\hline 85 & 8 & 87 & 88 & 89 & ஜ10 \\
\hline 89 & 83 & (2) & 81 & 80 & (19) \\
\hline 13 & (2) & 18 & (10) & (27) & 88 \\
\hline (12) & (12) & (10) & (99 & 68 & (6) \\
\hline (1) & (2) & (3) & 6 & 6 & ஏ \\
\hline 60 & 69 & 68 & (5) & 60 & 65 \\
\hline (49) & (10 & (1) & (2) & 63 & 69 \\
\hline 48 & (4) & (46) & 45 & (4) & 43 \\
\hline 1 & 당 & (2) & 0 & (41) & (42) \\
\hline & 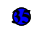 & D & - & ? & 0 \\
\hline & 0 & 0 & 0 & 0 & (1) \\
\hline 8 & 23 & (2) & (1) & (21) & (1) \\
\hline (3) & (1) & (1) & (10) & 0 & (1) \\
\hline (1) & 0 & (1) & (2) & (8) & (7) \\
\hline (1) & (2) & (3) & (4) & (5) & (6) \\
\hline
\end{tabular}

$13 \mathrm{~mm} \times 100 \mathrm{~mm}$ Tubes

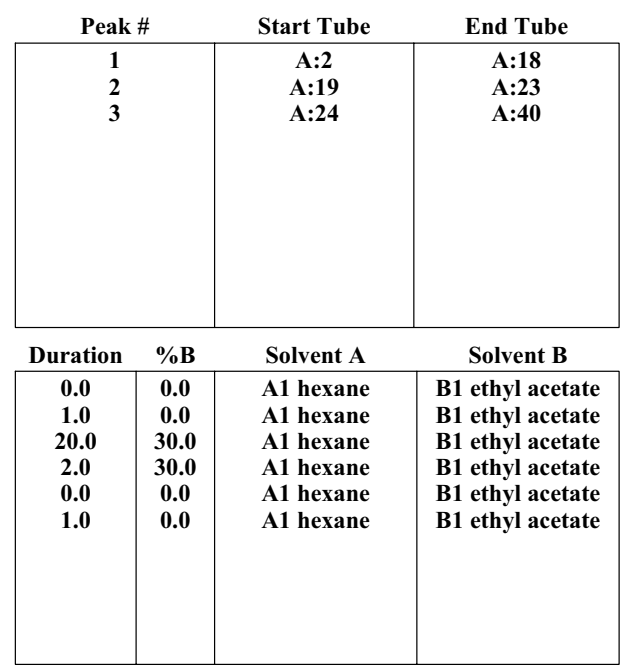

Page 1 of 1 


\section{Synthetic Methods}

\subsubsection{7 (2-(hexyloxy)-5-(phenylamino)phenyl)(phenyl)methanol: G2-100s9}

In a $20 \mathrm{~mL}$ vial, 2-(hexyloxy)-5-(phenylamino)benzaldehyde (G5-135s7,

$0.309 \mathrm{~g}, 0.90 \mathrm{mmol}$ ) was dissolved in anhydrous tetrahydrofuran (THF, $5 \mathrm{~mL}$ ). To the solution, phenylmagnesiumbromide (1.0 M in THF, $5 \mathrm{~mL}, 5 \mathrm{mmol})$ was added, and the reaction was stirred for an hour. Celite $(\sim 1 \mathrm{~g})$ and $1 \mathrm{M} \mathrm{NaOH}(5 \mathrm{~mL})$ was added to the reaction. The mixture was throughly mixed, filtered through a sintered glass funnel, and washed with ethyl acetate (EtOAc, $15 \mathrm{~mL}$ ). The filtrate was extracted with brine $(50 \mathrm{~mL})$. The brine phase was extracted with EtOAc $(30 \mathrm{~mL})$. The combined EtOAc phases were dried with $\mathrm{MgSO}_{4}$, and after filtering silica gel $(\sim 1 \mathrm{~g})$ was added to the filtrate. This mixture was concentrated by rotatory evaporation to a powder, which was used as a dry-load for silica chromatography (FIGURE 7.3). Fractions $17-49$ were combined and concentrated by rotatory evaporation $(0.2880 \mathrm{~g}, 89.5 \%$ yield $)$.

${ }^{1}$ H-NMR $\delta(\mathrm{ppm})\left(\right.$ DMSO-d $\left._{6}\right): 7.85$ (1 H, s), 7.29-7.35 (3 H, m), 7.23-7.29 (2 H, m), 7.11-7.17 (3 H, m), 6.88-6.94 (3 H, m), $6.83(1 \mathrm{H}, \mathrm{d}, \mathrm{J}=8.71 \mathrm{~Hz}), 6.66-6.72$ $(1 \mathrm{H}, \mathrm{m}), 5.95(1 \mathrm{H}, \mathrm{d}, \mathrm{J}=4.10 \mathrm{~Hz}), 5.64(1 \mathrm{H}, \mathrm{d}, \mathrm{J}=4.08 \mathrm{~Hz}), 3.87(2 \mathrm{H}, \mathrm{td}, \mathrm{J}=$ 6.28, $2.99 \mathrm{~Hz}), 1.62-1.72(2 \mathrm{H}, \mathrm{m}), 1.34-1.42(2 \mathrm{H}, \mathrm{m}), 1.26-1.34(4 \mathrm{H}, \mathrm{m}), 0.88$ (4 $\mathrm{H}, \mathrm{t}, \mathrm{J}=7.08 \mathrm{~Hz})$. 


\section{Synthetic Methods}

FIGURE 7.3: Chromatographic purification of G2-100s8.

Sample: grandy2-100

RediSep Column: Silica 40g

Flow Rate: $40 \mathrm{ml} / \mathrm{min}$

Equilibration Volume: 5.0 CV

Initial Waste: $0.0 \mathrm{CV}$

Air Purge: $1.0 \mathrm{~min}$

Solvent: A2 hexane

Solvent: B2 ethyl acetate
Rf 200 : OHSU COHEN RF200\#1

Peak Tube Volume: Max.

Non-Peak Tube Volume: Max.

Loading Type: Solid

Wavelength 1 (red): 254nm

Peak Width: 2 min

Threshold: $0.05 \mathrm{AU}$

Wavelength 2 (purple): 280nm
Wednesday 31 October 2012 07:39PM

All Wavelength (orange): $200 \mathrm{~nm}$ - 360nm

Peak Width: 2 min

Threshold: 0.20 AU

Run Notes:

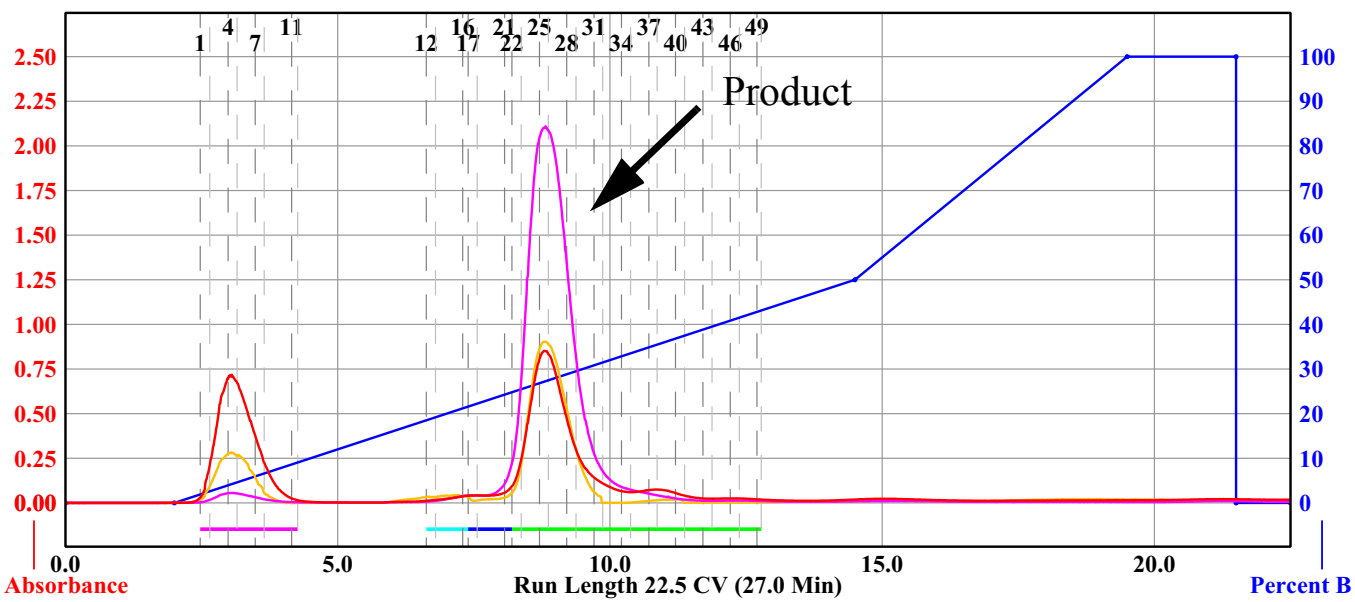

Rack A

\begin{tabular}{|c|c|c|c|c|c|}
\hline 108 & (1)7 & (106 & 105 & (104 & (103 \\
\hline 02 & $\mathscr{0}$ & (9) & 100 & (10) & (102 \\
\hline ஜ & (6) & Ө & (2) & (2) & (21) \\
\hline 8 & 80 & 87 & 88 & 89 & ஜD \\
\hline 8 & 83 & 82 & (11) & (10) & (19) \\
\hline$(3)$ & (2) & (1) & (16) & (12) & 18 \\
\hline (12) & (11) & (11) & 69 & 68 & (67) \\
\hline (1) & (2) & 63 & 9 & 67 & ø \\
\hline ஞ & 69 & 68 & 57 & 56 & 59 \\
\hline 49 & (10) & (1) & 62 & 63 & 69 \\
\hline 48 & (4) & (46) & (45) & (4) & (4) \\
\hline 37 & (B8 & (2) & (11) & (41) & (4) \\
\hline b & (35) & B) & 33 & (32) & (1) \\
\hline (2) & (26) & (27) & 28 & 29 & (10) \\
\hline (2) & (3) & (2) & 0 & D & - \\
\hline (1) & (1) & (1) & (1) & 0 & 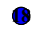 \\
\hline (12) & (1) & (1) & (2) & (8) & (1) \\
\hline (1) & (2) & (3) & (4) & (5) & (6) \\
\hline
\end{tabular}

$13 \mathrm{~mm} \times 100 \mathrm{~mm}$ Tubes

\begin{tabular}{|c|c|c|c|}
\hline \multicolumn{2}{|c|}{ Peak \# } & Start Tube & End Tube \\
\hline $\begin{array}{l}1 \\
2 \\
3 \\
4\end{array}$ & & $\begin{array}{c}\mathbf{A}: 1 \\
\mathbf{A}: 12 \\
\mathbf{A}: 17 \\
\mathbf{A}: 22\end{array}$ & $\begin{array}{l}\text { A:11 } \\
A: 16 \\
A: 21 \\
A: 49\end{array}$ \\
\hline Duration & $\%$ B & Solvent A & Solvent B \\
\hline $\begin{array}{c}0.0 \\
2.0 \\
12.5 \\
5.0 \\
2.0 \\
0.0 \\
1.0\end{array}$ & $\begin{array}{c}0.0 \\
0.0 \\
50.0 \\
100.0 \\
100.0 \\
0.0 \\
0.0\end{array}$ & $\begin{array}{l}\text { A2 hexane } \\
\text { A2 hexane } \\
\text { A2 hexane } \\
\text { A2 hexane } \\
\text { A2 hexane } \\
\text { A2 hexane } \\
\text { A2 hexane }\end{array}$ & $\begin{array}{l}\text { B2 ethyl acetate } \\
\text { B2 ethyl acetate } \\
\text { B2 ethyl acetate } \\
\text { B2 ethyl acetate } \\
\text { B2 ethyl acetate } \\
\text { B2 ethyl acetate } \\
\text { B2 ethyl acetate }\end{array}$ \\
\hline
\end{tabular}

Page 1 of 1 


\section{Synthetic Methods}

\subsubsection{8 (5-((4-fluorophenyl)amino)-2-(hexyloxy)phenyl)(phenyl)methanol: G2-}

$101 \mathrm{~s} 9$

In a $20 \mathrm{~mL}$ vial with a septum cap, 5-((4-fluorophenyl)amino)-2-(hexyloxy)benzaldehyde (G5-136s7, $0.228 \mathrm{~g}, 0.72 \mathrm{mmol}$ ) was dissolved in anhydrous tetrahydrofuran (THF, $5 \mathrm{~mL}$ ). To the solution, phenylmagnesiumbromide (1.0 $\mathrm{M}$ in THF, $5 \mathrm{~mL}, 5 \mathrm{mmol}$ ) was added, and the reaction was stirred for an hour. Celite $(\sim 1 \mathrm{~g})$ and $1 \mathrm{M} \mathrm{NaOH}(5 \mathrm{~mL})$ was added to the reaction. The mixture was throughly mixed, filtered through a sintered glass funnel, and washed with ethyl acetate $($ EtOAc, $15 \mathrm{~mL})$. The filtrate was extracted with brine $(50 \mathrm{~mL})$. The brine phase was extracted with EtOAc $(30 \mathrm{~mL})$. The combined EtOAc phases were dried with $\mathrm{MgSO}_{4}$, and after filtering silica gel $(\sim 1 \mathrm{~g})$ was added to the filtrate. This mixture was concentrated by rotatory evaporation to a powder, which was used as a dry-load for silica chromatography (FIGURE 7.4). Fractions 17-36 were combined and concentrated by rotatory evaporation $(0.2377 \mathrm{~g}, 83.9 \%$ yield $)$.

${ }^{1} \mathrm{H}-\mathrm{NMR} \delta(\mathrm{ppm})\left(\mathrm{DMSO}_{6}\right): 7.81(1 \mathrm{H}, \mathrm{s}), 7.30-7.34(2 \mathrm{H}, \mathrm{m}), 7.23-7.29$ (3

H, m), 7.14-7.19 (1 H, m), $7.00(2 \mathrm{H}, \mathrm{t}, \mathrm{J}=8.81 \mathrm{~Hz}), 6.85-6.93(3 \mathrm{H}, \mathrm{m}), 6.81(1 \mathrm{H}$, d, J = 8.76 Hz), $5.94(1 \mathrm{H}, \mathrm{d}, \mathrm{J}=4.09 \mathrm{~Hz}), 5.64(1 \mathrm{H}, \mathrm{d}, \mathrm{J}=4.09 \mathrm{~Hz}), 3.86(2 \mathrm{H}, \mathrm{td}$, $\mathrm{J}=6.32,3.30 \mathrm{~Hz}), 1.62-1.71(2 \mathrm{H}, \mathrm{m}), 1.33-1.42(2 \mathrm{H}, \mathrm{s}), 1.26-1.33(4 \mathrm{H}, \mathrm{m}), 0.88$ $(3 \mathrm{H}, \mathrm{t}, \mathrm{J}=6.58 \mathrm{~Hz})$. 


\section{Synthetic Methods}

FIGURE 7.4: Chromatographic purification of G2-101s8.

Sample: grandy2-101

RediSep Column: Silica 40g

Flow Rate: $40 \mathrm{ml} / \mathrm{min}$

Equilibration Volume: 5.0 CV

Initial Waste: $0.0 \mathrm{CV}$

Air Purge: $1.0 \mathrm{~min}$

Solvent: A2 hexane

Solvent: B2 ethyl acetate
Rf 200 : OHSU COHEN RF200\#1

Peak Tube Volume: Max.

Non-Peak Tube Volume: Max.

Loading Type: Solid

Wavelength 1 (red): 254nm

Peak Width: 2 min

Threshold: 0.05 AU

Wavelength 2 (purple): 280nm
Wednesday 31 October 2012 08:24PM

All Wavelength (orange): $200 \mathrm{~nm}-360 \mathrm{~nm}$

Peak Width: 2 min

Threshold: 0.20 AU

Run Notes:

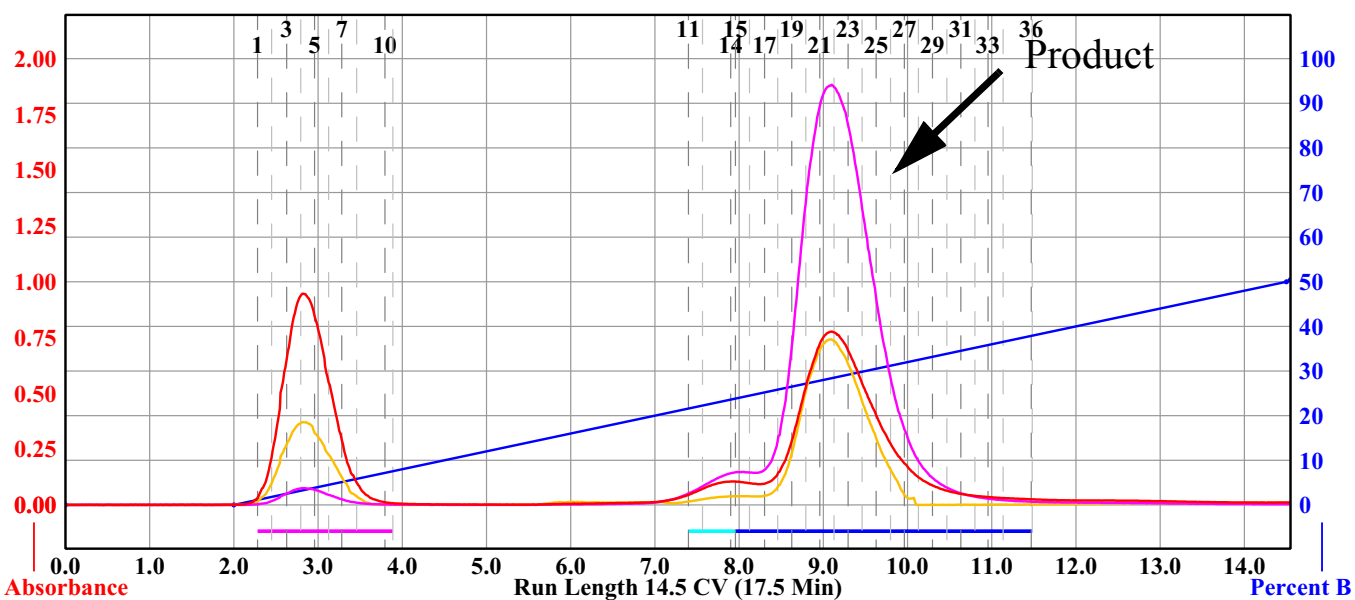

Rack A

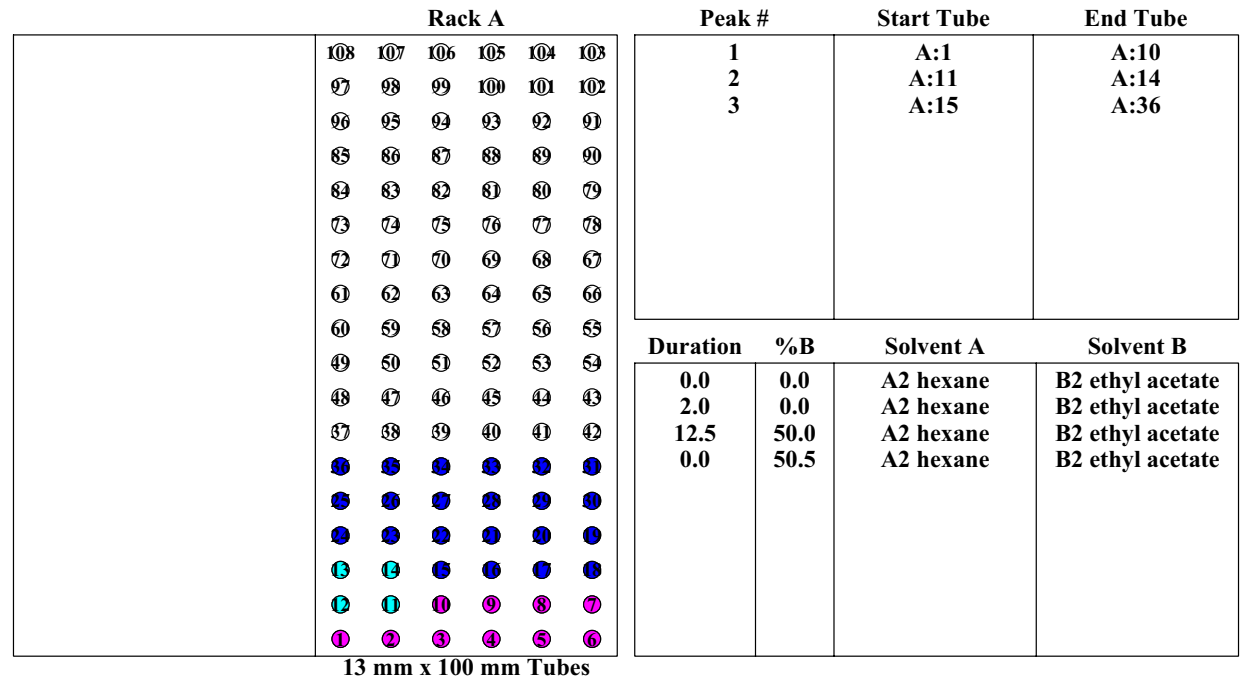

Page 1 of 1 


\section{Synthetic Methods}

\subsubsection{9 (5-((3-fluorophenyl)amino)-2-(hexyloxy)phenyl)(phenyl)methanol:}

\section{G2-102s9}

In a $20 \mathrm{~mL}$ vial with a septum cap, 5-((3-fluorophenyl)amino)-2-(hexy-

loxy)benzaldehyde (G5-137s7, $0.3480 \mathrm{~g}, 1.10 \mathrm{mmol})$ was dissolved in anhydrous tetrahydrofuran (THF, $5 \mathrm{~mL}$ ). To the solution, phenylmagnesiumbromide (1.0 M in THF, $5 \mathrm{~mL}, 5 \mathrm{mmol}$ ) was added, and the reaction was stirred for an hour. Celite $(\sim 1 \mathrm{~g})$ and $1 \mathrm{M} \mathrm{NaOH}(5 \mathrm{~mL})$ was added to the reaction. The mixture was throughly mixed, filtered through a sintered glass funnel, and washed with ethyl acetate $($ EtOAc, $15 \mathrm{~mL})$. The filtrate was extracted with brine $(50 \mathrm{~mL})$. The brine phase was extracted with EtOAc $(30 \mathrm{~mL})$. The combined EtOAc phases were dried with $\mathrm{MgSO}_{4}$, and after filtering, silica gel $(\sim 1 \mathrm{~g})$ was added to the filtrate. This mixture was concentrated by rotatory evaporation to a powder, which was used as a dry-load for silica chromatography (FIGURE 7.5). Fractions 17-28 were combined and concentrated by rotatory evaporation $(0.3374 \mathrm{~g}, 78.0 \%$ yield $)$.

${ }^{1} \mathrm{H}-\mathrm{NMR} \delta(\mathrm{ppm})\left(\mathrm{DMSO}_{6}\right): 8.14(1 \mathrm{H}, \mathrm{s}), 7.39-7.41(2 \mathrm{H}, \mathrm{m}), 7.31-7.36(4$ H, m), 7.13-7.15 (1 H, m), 6.95 (1 H, dd, J = 8.68, $2.80 \mathrm{~Hz}), 6.86(1 \mathrm{H}, \mathrm{d}, \mathrm{J}=8.76$ Hz), 6.66-6.71 (1 H, m), 6.60 (1 H, dt, J = 12.24, $2.31 \mathrm{~Hz}), 6.45(1 \mathrm{H}, \mathrm{td}, \mathrm{J}=8.20$, $2.48 \mathrm{~Hz}), 5.95(1 \mathrm{H}, \mathrm{d}, \mathrm{J}=4.17 \mathrm{~Hz}), 5.68(1 \mathrm{H}, \mathrm{J}=4.16 \mathrm{~Hz}), 3.89(2 \mathrm{H}, \mathrm{td}, \mathrm{J}=6.39$, $2.78 \mathrm{~Hz}), 1.64-1.72(2 \mathrm{H}, \mathrm{m}), 1.33-1.42(2 \mathrm{H}, \mathrm{m}), 1.27-1.33(4 \mathrm{H}, \mathrm{m}), 0.88(3 \mathrm{H}, \mathrm{t}$, $\mathrm{J}=6.64 \mathrm{~Hz})$. 


\section{Synthetic Methods}

FIGURE 7.5: Chromatographic purification of G2-102s8.

Sample: grandy2-102s8

RediSep Column: Silica 40g

Flow Rate: $40 \mathrm{ml} / \mathrm{min}$

Equilibration Volume: 5.0 CV

Initial Waste: $0.0 \mathrm{CV}$

Air Purge: $1.0 \mathrm{~min}$

Solvent: A2 hexane

Solvent: B2 ethyl acetate
Rf 200 : OHSU COHEN RF200\#1

Peak Tube Volume: Max.

Non-Peak Tube Volume: Max.

Loading Type: Solid

Wavelength 1 (red): 254nm

Peak Width: 2 min

Threshold: 0.05 AU

Wavelength 2 (purple): 280nm
Wednesday 31 October 2012 08:55PM

All Wavelength (orange): 200nm - 360nm

Peak Width: 2 min

Threshold: 0.20 AU

Run Notes:

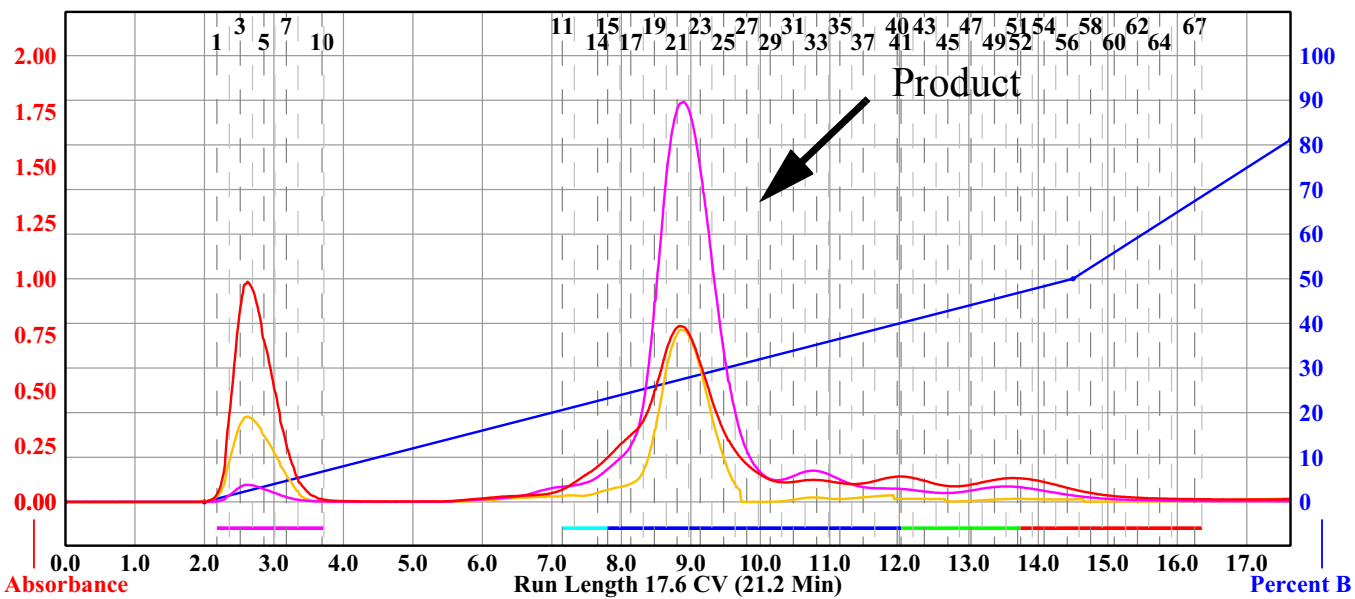

Rack A

\begin{tabular}{|c|c|c|c|c|c|}
\hline 108 & (10) & 106 & 105 & (104 & 1(13) \\
\hline (2) & $\mathscr{B}$ & $\mathscr{W 9}$ & (100 & (10) & 102 \\
\hline שூ & (S) & Ө & (2) & (2) & (11) \\
\hline 85 & $\mathbb{B}$ & 87 & 88 & 89 & DO \\
\hline 89 & 83 & 82 & (11) & (10) & (1) \\
\hline (13) & (2) & (2) & (2) & (12) & 88 \\
\hline (2) & (11) & (11) & 69 & 68 & (1) \\
\hline (1) & (2) & 6 & $\theta$ & 6 & 6 \\
\hline (1) & (2) & 68 & 5 & 6 & 6 \\
\hline (4) & (10) & (1) & (2) & 63 & 6 \\
\hline (48) & (4) & (46) & (45) & (4) & (43) \\
\hline 6 & (2) & 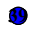 & (1) & (41) & (4) \\
\hline & & D & 0 & 0 & 0 \\
\hline & - & & & & \\
\hline (1) & (1) & 0 & 0 & 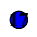 & 0 \\
\hline (1) & (11) & (1) & (9) & (8) & (1) \\
\hline (1) & (2) & (3) & (4) & (5) & (6) \\
\hline
\end{tabular}

$13 \mathrm{~mm} \times 100 \mathrm{~mm}$ Tubes

\begin{tabular}{|c|c|c|c|}
\hline \multicolumn{2}{|c|}{ Peak \# } & Start Tube & End Tube \\
\hline $\begin{array}{l}1 \\
2 \\
3 \\
4 \\
5\end{array}$ & & $\begin{array}{c}A: 1 \\
A: 11 \\
A: 15 \\
A: 41 \\
A: 52\end{array}$ & $\begin{array}{l}A: 10 \\
A: 14 \\
A: 40 \\
A: 51 \\
A: 67\end{array}$ \\
\hline Duration & $\%$ В & Solvent A & Solvent B \\
\hline $\begin{array}{c}0.0 \\
2.0 \\
12.5 \\
3.1\end{array}$ & $\begin{array}{c}0.0 \\
0.0 \\
50.0 \\
81.1\end{array}$ & $\begin{array}{l}\text { A2 hexane } \\
\text { A2 hexane } \\
\text { A2 hexane } \\
\text { A2 hexane }\end{array}$ & $\begin{array}{l}\text { B2 ethyl acetate } \\
\text { B2 ethyl acetate } \\
\text { B2 ethyl acetate } \\
\text { B2 ethyl acetate }\end{array}$ \\
\hline
\end{tabular}

Page 1 of 1 


\section{Synthetic Methods}

\subsubsection{Hexyl 2-(hexyloxy)-5-(phenylamino)benzoate: G5-059s9}

In a round bottom flask equipped with a drying tube open to the air, hexyl 5amino-2-(hexyloxy)benzoate (G5-047s9, 82.95 mass\% w/EtOAc, 3 mmol, 1.163 g), phenylboronic acid (12 mmol, $1.4692 \mathrm{~g}), \mathrm{Cu}(\mathrm{OAc})_{2}(6.9 \mathrm{mmol}, 1.253 \mathrm{~g})$, triethylamine (22.5 mmol, $2.277 \mathrm{~g}, 3.136 \mathrm{~mL})$, powdered vacuum oven dried $4 \AA$ molecular sieves (3 g) and anhydrous dichloromethane (DCM, $30 \mathrm{~mL}$ ) were combined. After stirring for 2 days, the reaction mixture was filtered through packed celite ( $2 \mathrm{~cm}$ in a $60 \mathrm{~mL}$ sintered glass funnel), and washed with DCM. The filtrate was extracted with $5 \mathrm{M} \mathrm{NH}_{4} \mathrm{OH}(2 \times 75 \mathrm{~mL})$ followed by an extraction with water $(50 \mathrm{~mL})$. The DCM phase was dried with $\mathrm{MgSO}_{4}$, and after filtering, silica gel ( $\sim 5 \mathrm{~g})$ was added to the filtrate. This mixture was concentrated by rotatory evaporation to a powder, which was used as a dry-load for silica chromatography (FIGURE 7.6). Fractions $15-24$ were combined and concentrated by rotatory evaporation $(0.492 \mathrm{~g}, 41.3 \%$ yield $)$.

Notes: In climates drier than Portland, Oregon, $1 \mathrm{~g}$ of powdered molecular sieves should suffice. Also, if one were to repeat this experiment at the same scale and using $3 \mathrm{~g}$ of powdered molecular sieves, increasing the amount of DCM used would improve the stirrability of the reaction.

${ }^{1} \mathrm{H}-\mathrm{NMR} \delta(\mathrm{ppm})\left(\mathrm{DMSO}_{6}\right): 8.01(1 \mathrm{H}, \mathrm{s}), 7.36(1 \mathrm{H}, \mathrm{d}, \mathrm{J}=2.90 \mathrm{~Hz}), 7.16-$

$7.24(3 \mathrm{H}, \mathrm{m}), 7.05(1 \mathrm{H}, \mathrm{d}, \mathrm{J}=8.92 \mathrm{~Hz}), 6.95(2 \mathrm{H}, \mathrm{dt}, \mathrm{J}=7.96,1.12 \mathrm{~Hz}), 6.77$ (1 


\section{Synthetic Methods}

$\mathrm{H}, \mathrm{tt}, \mathrm{J}=7.30,1.00 \mathrm{~Hz}), 4.20(2 \mathrm{H}, \mathrm{t}, \mathrm{J}=6.47 \mathrm{~Hz}), 3.96(2 \mathrm{H}, \mathrm{t}, \mathrm{J}=6.32 \mathrm{~Hz}), 1.60-$

$1.74(4 \mathrm{H}, \mathrm{m}), 1.36-1.49(4 \mathrm{H}, \mathrm{m}), 1.23-1.36(8 \mathrm{H}, \mathrm{m}), 0.82-0.93(6 \mathrm{H}, \mathrm{m})$.

${ }^{13} \mathrm{C}-\mathrm{NMR} \delta(\mathrm{ppm})\left(\mathrm{DMSO}_{6}\right)$ ): 166.1, 151.8, 144.2, 136.1, 129.2, 123.1,

$121.2,120.1,119.0,115.5,115.1,68.8,64.4,31.0,30.9,28.8,28.2,25.2(2 \mathrm{C})$,

$22.1,22.0,14.1,13.9$. 


\section{Synthetic Methods}

FIGURE 7.6: Chromatographic purification of G5-059s7.

Sample: grandy5-059s7

RediSep Column: Silica 24g

Flow Rate: 35 ml/min

Equilibration Volume: 5.0 CV

Initial Waste: $0.0 \mathrm{CV}$

Air Purge: $1.0 \mathrm{~min}$

Solvent: A2 hexane

Solvent: B2 ethyl acetate
Rf 200 : OHSU COHEN RF200\#1

Peak Tube Volume: Max.

Non-Peak Tube Volume: Max.

Loading Type: Solid (Pause)

Wavelength 1 (red): 254nm

Peak Width: 1 min

Threshold: $0.05 \mathrm{AU}$

Wavelength 2 (purple): 280nm

Run Notes: self-packed column, $\mathbf{5 0 g}$

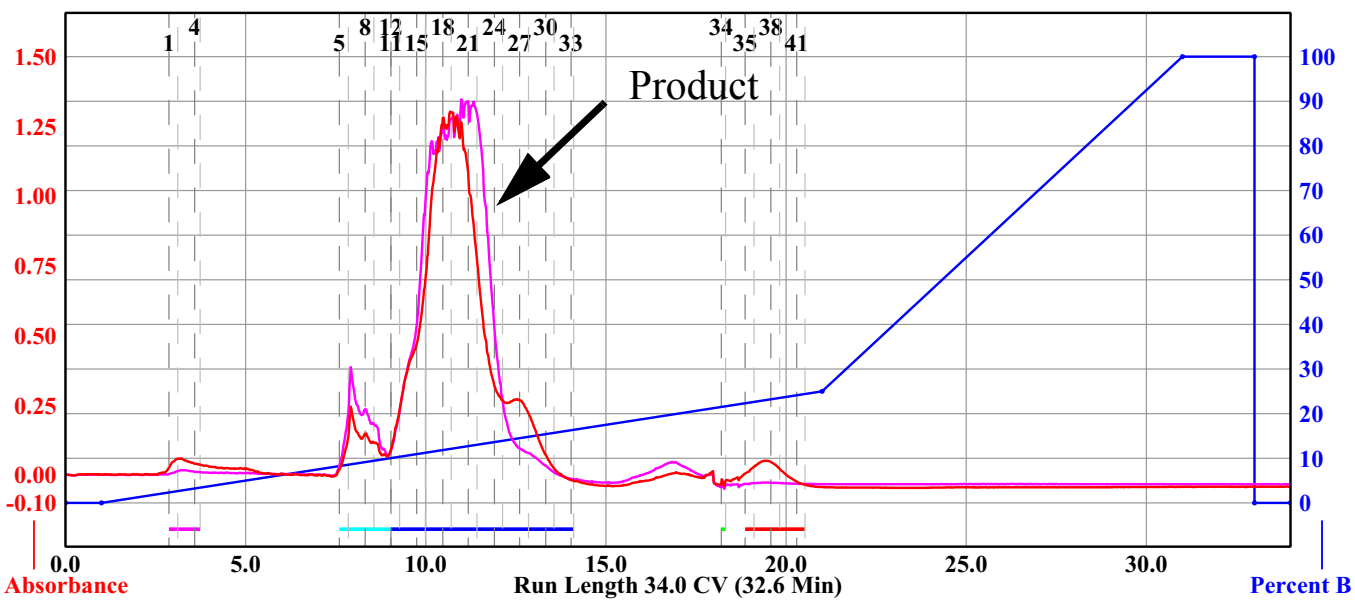

Rack A

\begin{tabular}{|c|c|c|c|c|c|}
\hline 108 & (107 & 106 & 105 & (14) & (103 \\
\hline (2) & 8 & (29) & 100 & (10) & (102 \\
\hline ש6 & (25) & (9) & (2) & (22) & (1) \\
\hline 85 & 80 & 87 & 88 & 89 & $\mathscr{D}$ \\
\hline 89 & 83 & (2) & (11) & (10) & (19) \\
\hline 13 & (2) & (2) & (20) & (2) & 18 \\
\hline (12) & (11) & (11) & (9) & 68 & (6) \\
\hline (1) & (2) & 63 & (9) & 65 & 60 \\
\hline (10 & 69 & 68 & (57) & 50 & 69 \\
\hline 49 & (10) & (1) & (2) & 63 & 69 \\
\hline 48 & (47) & (46) & (45) & 4 & (43) \\
\hline ש & (3) & (3) & (1) & (1) & (42) \\
\hline 2) & (3) & (3) & 0 & 0 & 0 \\
\hline 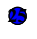 & 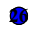 & 0 & • & - & - \\
\hline 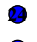 & - & & & & \\
\hline 0 & 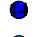 & 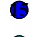 & ? & ? & \\
\hline 0 & (1) & (1) & (D) & (8) & (7) \\
\hline (1) & (2) & (3) & (4) & (5) & (6) \\
\hline
\end{tabular}

$13 \mathrm{~mm} \times 100 \mathrm{~mm}$ Tubes

\begin{tabular}{|c|c|c|c|}
\hline \multicolumn{2}{|c|}{ Peak \# } & Start Tube & End Tube \\
\hline $\begin{array}{l}1 \\
2 \\
3 \\
4 \\
5\end{array}$ & & $\begin{array}{c}A: 1 \\
A: 5 \\
A: 12 \\
A: 34 \\
A: 35\end{array}$ & $\begin{array}{c}A: 4 \\
A: 11 \\
A: 33 \\
A: 34 \\
A: 41\end{array}$ \\
\hline Duration & $\%$ B & Solvent A & Solvent B \\
\hline \begin{tabular}{c|}
0.0 \\
1.0 \\
20.0 \\
10.0 \\
2.0 \\
0.0 \\
1.0
\end{tabular} & $\begin{array}{c}0.0 \\
0.0 \\
25.0 \\
100.0 \\
100.0 \\
0.0 \\
0.0\end{array}$ & $\begin{array}{l}\text { A2 hexane } \\
\text { A2 hexane } \\
\text { A2 hexane } \\
\text { A2 hexane } \\
\text { A2 hexane } \\
\text { A2 hexane } \\
\text { A2 hexane }\end{array}$ & $\begin{array}{l}\text { B2 ethyl acetate } \\
\text { B2 ethyl acetate } \\
\text { B2 ethyl acetate } \\
\text { B2 ethyl acetate } \\
\text { B2 ethyl acetate } \\
\text { B2 ethyl acetate } \\
\text { B2 ethyl acetate }\end{array}$ \\
\hline
\end{tabular}

Page 1 of 1 


\section{Synthetic Methods}

\subsubsection{Hexyl 5-((4-fluorophenyl)amino)-2-(hexyloxy)benzoate: G5-060s9}

In a $20 \mathrm{~mL}$ vial with a septum cap and equipped with a drying tube open to the air, hexyl 5-amino-2-(hexyloxy)benzoate (G5-047s9, 82.95 mass\% w/EtOAc, 1 mmol, $0.3960 \mathrm{~g})$, 4-fluorophenylboronic acid (4 mmol, $0.5629 \mathrm{~g}), \mathrm{Cu}(\mathrm{OAc})_{2}(2.3$ mmol, $0.313 \mathrm{~g})$, triethylamine $(7.5 \mathrm{mmol}, 0.759 \mathrm{~g}, 1.045 \mathrm{~mL})$, powdered vacuum oven dried $4 \AA$ molecular sieves ( $1 \mathrm{~g}$ ) and anhydrous dichloromethane (DCM, 10 $\mathrm{mL}$ ) were combined. After stirring for 2 days, the reaction mixture was filtered through packed celite ( $2 \mathrm{~cm}$ in a $60 \mathrm{~mL}$ sintered glass funnel), and washed with DCM. The filtrate was extracted with $5 \mathrm{M} \mathrm{NH}_{4} \mathrm{OH}(2 \times 75 \mathrm{~mL})$ followed by an extraction with water $(50 \mathrm{~mL})$. The DCM phase was dried with $\mathrm{MgSO}_{4}$, and after filtering, silica gel ( $\sim 5 \mathrm{~g})$ was added to the filtrate. This mixture was concentrated by rotatory evaporation to a powder, which was used as a dry-load for silica chromatography (FIGURE 7.7). Fractions 7-20 were combined and concentrated by rotatory evaporation $(0.1435 \mathrm{~g}, 34.5 \%$ yield $)$.

Notes: In climates drier than Portland, Oregon, $0.3 \mathrm{~g}$ of powdered molecular sieves should suffice. Also, if one were to repeat this experiment using $1 \mathrm{~g}$ of powdered molecular sieves, increasing the amount of DCM used would improve the stirrability of the reaction.

${ }^{1} \mathrm{H}-\mathrm{NMR} \delta(\mathrm{ppm})\left(\mathrm{DMSO}_{6}\right): 7.96(1 \mathrm{H}, \mathrm{s}), 7.30(1 \mathrm{H}, \mathrm{d}, \mathrm{J}=2.91 \mathrm{~Hz}), 7.18(1$ H, dd, J = 8.88, 2.93 Hz), 7.01-7.09 (3 H, m), 6.93-6.99 (2 H, m), 4.19 (2 H, t, J = 


\section{Synthetic Methods}

$6.53 \mathrm{~Hz}), 3.95(2 \mathrm{H}, \mathrm{t}, \mathrm{J}=6.33 \mathrm{~Hz}), 1.60-1.74(4 \mathrm{H}, \mathrm{m}), 1.35-1.48(4 \mathrm{H}, \mathrm{m}), 1.26-$

$1.34(8 \mathrm{H}, \mathrm{m}), 0.83-0.91(6 \mathrm{H}, \mathrm{m})$.

${ }^{13} \mathrm{C}-\mathrm{NMR} \delta(\mathrm{ppm})\left(\right.$ DMSO-d $\left._{6}\right): 166.1,(157.2 \& 154.8(1 \mathrm{C}, \mathrm{d}, \mathrm{J}=235.40 \mathrm{~Hz})$, 151.6, 140.6, 136.6, 122.4, 121.3, 119.4, 117.45 (1 C, d, J = 7.58 Hz), 115.7 (1 C, d, $\mathrm{J}=22.33 \mathrm{~Hz}), 115.3,68.9,64.4,31.0,30.9,28.8,28.2,25.2,22.4,22.1,22.0$, $13.9(2 \mathrm{C})$. 


\section{Synthetic Methods}

FIGURE 7.7: Chromatographic purification of G5-060s7.

Sample: grandy5-060s7

RediSep Column: Silica 40g

Flow Rate: $40 \mathrm{ml} / \mathrm{min}$

Equilibration Volume: 5.0 CV

Initial Waste: $0.0 \mathrm{CV}$

Air Purge: $1.0 \mathrm{~min}$

Solvent: A2 hexane

Solvent: B2 ethyl acetate
Rf 200 : OHSU COHEN RF200\#1

Peak Tube Volume: Max.

Non-Peak Tube Volume: Max.

Loading Type: Solid (Pause)

Wavelength 1 (red): 254nm

Peak Width: 2 min

Threshold: $0.05 \mathrm{AU}$

Wavelength 2 (purple): 280nm

Run Notes: self-packed column, $\mathbf{5 0 g}$

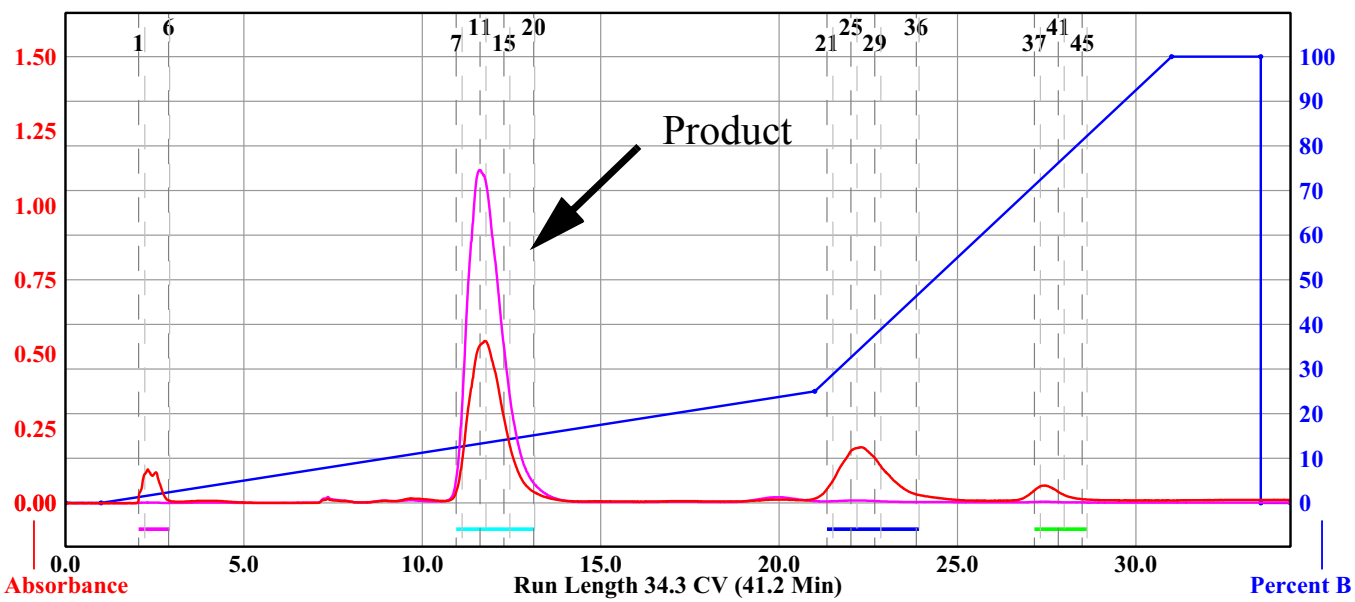

Rack A

\begin{tabular}{|c|c|c|c|c|c|}
\hline 108 & (10) & 106 & 105 & (104 & 1(13) \\
\hline (2) & $\mathscr{B}$ & $\mathscr{W 9}$ & (100 & (10) & 102 \\
\hline שூ & (S) & Ө & (2) & (2) & (11) \\
\hline 8 & $\mathbb{B g}$ & 87 & 88 & 89 & DQ \\
\hline 89 & 83 & 82 & (11) & (10) & (1) \\
\hline 83 & (2) & (2) & (2) & (12) & 88 \\
\hline (2) & (11) & (11) & 69 & 68 & (67) \\
\hline (1) & (2) & 63 & 8 & 6 & ஐ \\
\hline (10) & 69 & 68 & 57 & 60 & 69 \\
\hline (4) & (10) & (D) & 62 & 63 & 69 \\
\hline (48) & (47) & (46) & (4) & (4) & (4) \\
\hline (3) & $B$ & (3) & (4) & (41) & (4) \\
\hline 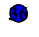 & 0 & - & ? & - & 0 \\
\hline & 8 & & 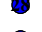 & & \\
\hline & 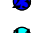 & 0 & 0 & (11) & 19 \\
\hline (1) & (1) & (1) & (1) & 10 & 8 \\
\hline (12) & (11) & (11) & (9) & (8) & (7) \\
\hline (1) & (2) & (3) & (4) & (5) & (6) \\
\hline
\end{tabular}

$13 \mathrm{~mm} \times 100 \mathrm{~mm}$ Tubes

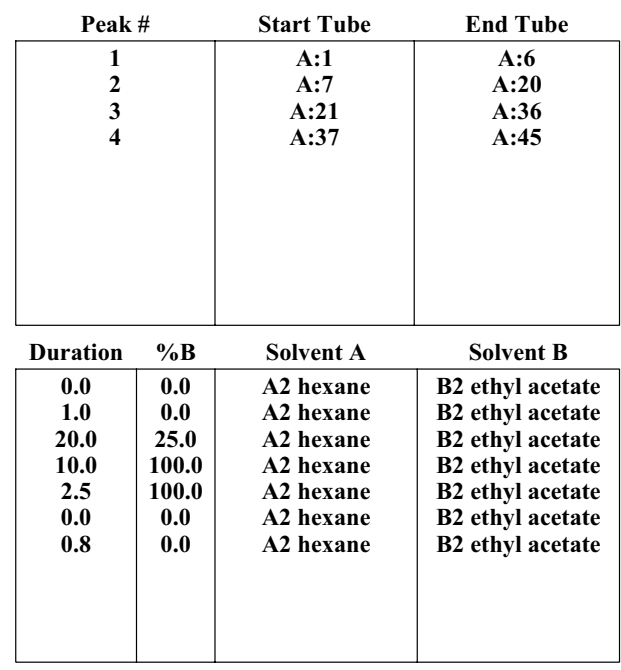

Page 1 of 1 


\section{Synthetic Methods}

\subsubsection{Hexyl 5-((3-fluorophenyl)amino)-2-(hexyloxy)benzoate: G5-061s9}

In a $20 \mathrm{~mL}$ vial with a septum cap and equipped with a drying tube open to the air, hexyl 5-amino-2-(hexyloxy)benzoate (G5-047s9, 82.95 mass\% w/EtOAc, 1 mmol, $0.3909 \mathrm{~g}), 3$-fluorophenylboronic acid (4 mmol, $0.5549 \mathrm{~g}), \mathrm{Cu}(\mathrm{OAc})_{2}(2.3$ mmol, $0.313 \mathrm{~g})$, triethylamine $(7.5 \mathrm{mmol}, 0.759 \mathrm{~g}, 1.045 \mathrm{~mL})$, powdered vacuum oven dried $4 \AA$ molecular sieves ( $1 \mathrm{~g}$ ) and anhydrous dichloromethane (DCM, 10 $\mathrm{mL}$ ) were combined. After stirring for 2 days, the reaction mixture was filtered through packed celite ( $2 \mathrm{~cm}$ in a $60 \mathrm{~mL}$ sintered glass funnel), and washed with DCM. The filtrate was extracted with $5 \mathrm{M} \mathrm{NH}_{4} \mathrm{OH}(2 \times 75 \mathrm{~mL})$ followed by an extraction with water $(50 \mathrm{~mL})$. The DCM phase was dried with $\mathrm{MgSO}_{4}$, and after filtering, silica gel ( $\sim 5 \mathrm{~g})$ was added to the filtrate. This mixture was concentrated by rotatory evaporation to a powder, which was used as a dry-load for silica chromatography (FIGURE 7.8). Fractions 7-21 were combined and concentrated by rotatory evaporation $(0.1487 \mathrm{~g}, 35.8 \%$ yield $)$.

Notes: In climates drier than Portland, Oregon, $0.3 \mathrm{~g}$ of powdered molecular sieves should suffice. Also, if one were to repeat this experiment using $1 \mathrm{~g}$ of powdered molecular sieves, increasing the amount of DCM used would improve the stirrability of the reaction.

${ }^{1} \mathrm{H}_{-N M R} \delta(\mathrm{ppm})\left(\right.$ DMSO-d $\left._{6}\right): 8.27(1 \mathrm{H}, \mathrm{s}), 7.38(1 \mathrm{H}, \mathrm{d}, \mathrm{J}=2.88 \mathrm{~Hz}), 7.28(1$ $\mathrm{H}, \mathrm{dd}, \mathrm{J}=8.88,2.88 \mathrm{~Hz}), 7.16-7.23(1 \mathrm{H}, \mathrm{m}), 7.09$ (1 H, d, J = 8.96 Hz), 6.71-6.75 $(1 \mathrm{H}, \mathrm{m}), 6.64(1 \mathrm{H}, \mathrm{dt}, \mathrm{J}=11.92,2.24 \mathrm{~Hz}), 6.49-6.55(1 \mathrm{H}, \mathrm{m}), 4.20(2 \mathrm{H}, \mathrm{t}, \mathrm{J}=$ 


\section{Synthetic Methods}

$6.44 \mathrm{~Hz}), 3.98(2 \mathrm{H}, \mathrm{t}, \mathrm{J}=6.32 \mathrm{~Hz}), 1.61-1.75(4 \mathrm{H}, \mathrm{m}), 1.35-1.49(4 \mathrm{H}, \mathrm{m}), 1.26-$

$1.35(8 \mathrm{H}, \mathrm{m}), 0.83-0.93(6 \mathrm{H}, \mathrm{m})$.

${ }^{13} \mathrm{C}-\mathrm{NMR} \delta(\mathrm{ppm})\left(\right.$ DMSO-d $\left._{6}\right): 166.0,163.2(1 \mathrm{C}, \mathrm{d}, \mathrm{J}=240.83 \mathrm{~Hz}), 152.6$, $146.7(1 \mathrm{C}, \mathrm{d}, \mathrm{J}=10.86 \mathrm{~Hz}), 134.8,130.7(1 \mathrm{C}, \mathrm{d}, \mathrm{J}=9.87 \mathrm{~Hz}), 124.5,121.6$, 121.2, 115.0, 110.7, 104.7 (1 C, d, J = 21.35 Hz), $100.9(1 \mathrm{C}, \mathrm{d}, \mathrm{J}=24.99 \mathrm{~Hz})$, $68.7,64.5,31.0,30.9,28.8,28.2,25.2(2 \mathrm{C}), 22.1,22.0,13.9$ (2 C). 


\section{Synthetic Methods}

FIGURE 7.8: Chromatographic purification of G5-061s7.

Sample: grandy5-061s7

RediSep Column: Silica 40g

Flow Rate: $40 \mathrm{ml} / \mathrm{min}$

Equilibration Volume: 5.0 CV

Initial Waste: $0.0 \mathrm{CV}$

Air Purge: $1.0 \mathrm{~min}$

Solvent: A2 hexane

Solvent: B2 ethyl acetate
Rf 200 : OHSU COHEN RF200\#1

Peak Tube Volume: Max.

Non-Peak Tube Volume: Max.

Loading Type: Solid

Wavelength 1 (red): 254nm

Peak Width: 2 min

Threshold: 0.05 AU

Wavelength 2 (purple): 280nm
Wednesday 22 August 2012 05:13PM

Run Notes: self-packed column, $50 \mathrm{~g}$

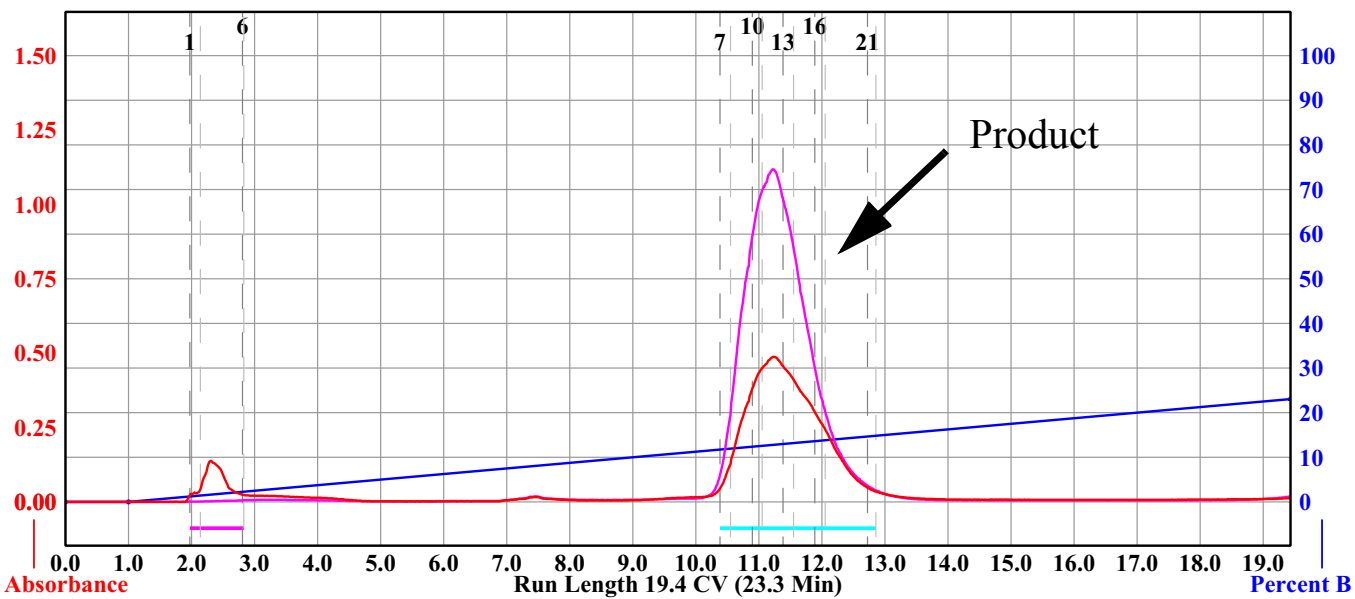

Rack A

\begin{tabular}{|c|c|c|c|c|c|}
\hline 108 & (10) & 106 & 105 & (194 & 1(13) \\
\hline (2) & $\mathscr{\theta}$ & $\mathscr{W 9}$ & (100 & (10) & 102 \\
\hline ஜ & (2) & $\mathscr{\theta}$ & (23 & (2) & (11) \\
\hline 8 & 8 & 87 & 88 & 89 & DQ \\
\hline 8 & 83 & 82 & (11) & (10) & (1) \\
\hline$(3)$ & (2) & (2) & (2) & (12) & 88 \\
\hline (12) & (11) & (11) & 69 & 68 & (67) \\
\hline (1) & (2) & 63 & 6 & 6 & ஞ \\
\hline (1) & 59 & 68 & 57 & 60 & 69 \\
\hline (49 & (10) & (D) & 62 & 63 & 69 \\
\hline 48 & (47) & (46) & 45 & $\mathbb{4}$ & (43) \\
\hline (17) & B8 & (9) & (40) & (41) & (42) \\
\hline BO & B5 & (3) & B3 & (32) & (11) \\
\hline (2) & (26) & (27) & 88 & 29 & (10) \\
\hline 29 & 23 & (2) & (11) & (21) & (1) \\
\hline (1) & (1) & (1) & (1) & 10 & $\mathbb{B}$ \\
\hline (12) & (11) & (11) & (9) & (8) & (7) \\
\hline (1) & (2) & (3) & (4) & (5) & (6) \\
\hline
\end{tabular}

$13 \mathrm{~mm} \times 100 \mathrm{~mm}$ Tubes

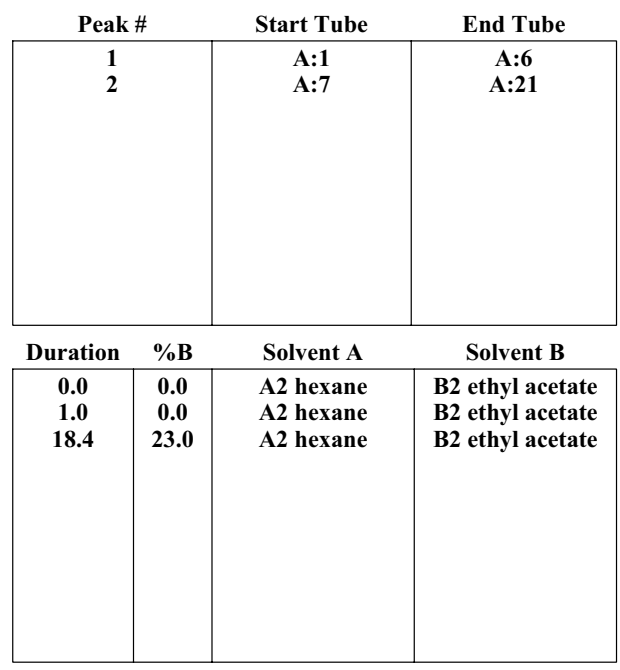

Page 1 of 1 


\section{Synthetic Methods}

\subsubsection{3 (2-(hexyloxy)-5-(phenylamino)phenyl)(pyrrolidin-1-yl)methanone: G5-} $112 \mathrm{~s} 5$

In a $20 \mathrm{~mL}$ vial with a septum cap and equipped with a drying tube open to the air, (5-amino-2-(hexyloxy)phenyl)(pyrrolidin-1-yl)methanone (G5-106s7, 0.99 mmol, $0.2880 \mathrm{~g})$, phenylboronic acid (1.5 mmol, $0.1859 \mathrm{~g}), \mathrm{Cu}(\mathrm{OAc})_{2}(2.3 \mathrm{mmol}$, $0.313 \mathrm{~g})$, triethylamine $(7.5 \mathrm{mmol}, 0.759 \mathrm{~g}, 1.045 \mathrm{~mL})$, powdered vacuum oven dried $4 \AA$ molecular sieves ( $1 \mathrm{~g})$ and anhydrous dichloromethane (DCM, $10 \mathrm{~mL}$ ) were combined. After stirring for 4 days, the reaction mixture was filtered through a cotton ball packed into a $10 \mathrm{~mL}$ syringe body, and washed with DCM. Silica gel ( $\sim 5 \mathrm{~g})$ was added to the filtrate, and the resulting mixture was concentrated by rotatory evaporation to a powder, which was used as a dry-load for silica chromatography (FIGURE 7.9). Fractions 1-19 were combined and concentrated by rotatory evaporation to an oil that later crystallized ( $0.1646 \mathrm{~g}, 44.9 \%$ yield).

Notes: In climates drier than Portland, Oregon, $0.3 \mathrm{~g}$ of powdered molecular sieves should suffice. Also, if one were to repeat this experiment using $1 \mathrm{~g}$ of powdered molecular sieves, increasing the amount of DCM used would improve the stirrability of the reaction.

\footnotetext{
${ }^{1} \mathrm{H}-\mathrm{NMR} \delta(\mathrm{ppm})\left(\mathrm{DMSO}_{6}\right): 7.92(1 \mathrm{H}, \mathrm{s}), 7.16-7.22(2 \mathrm{H}, \mathrm{m}), 7.07$ (1 H, dd, $\mathrm{J}=8.82,2.78 \mathrm{~Hz}), 6.92-7.00(3 \mathrm{H}, \mathrm{m}), 6.87(1 \mathrm{H}, \mathrm{d}, \mathrm{J}=2.76 \mathrm{~Hz}), 6.72-6.78(1 \mathrm{H}$, m), $3.94(2 \mathrm{H}, \mathrm{t}, \mathrm{J}=6.23 \mathrm{~Hz}), 3.42(2 \mathrm{H}, \mathrm{t}, \mathrm{J}=6.68 \mathrm{~Hz}), 3.11-3.21(2 \mathrm{H}, \mathrm{m}), 1.74-$
} 


\section{Synthetic Methods}

1.89 (4 H, m), 1.60-1.69 (2 H, m), 1.35-1.44 (2 H, s), 1.24-1.35 (4 H, m), 0.88 (3

$\mathrm{H}, \mathrm{t}, \mathrm{J}=6.62 \mathrm{~Hz})$. 


\section{Synthetic Methods}

FIGURE 7.9: Chromatographic purification of G5-112s4.

Sample: grandy5-112s4

RediSep Column: Silica 40g

Flow Rate: $40 \mathrm{ml} / \mathrm{min}$

Equilibration Volume: $240.0 \mathrm{ml}$

Initial Waste: $0.0 \mathrm{ml}$

Air Purge: $1.0 \mathrm{~min}$

Solvent: A2 hexane

Solvent: B2 ethyl acetate
Rf 200 : OHSU COHEN RF200\#1

Monday 08 October 2012 02:32PM

Peak Tube Volume: Max.

Non-Peak Tube Volume: Max.

Loading Type: Solid

Wavelength 1 (red): $254 \mathrm{~nm}$

Peak Width: 2 min

Threshold: 0.05 AU

Wavelength 2 (purple): 280nm

Run Notes: self-packed column

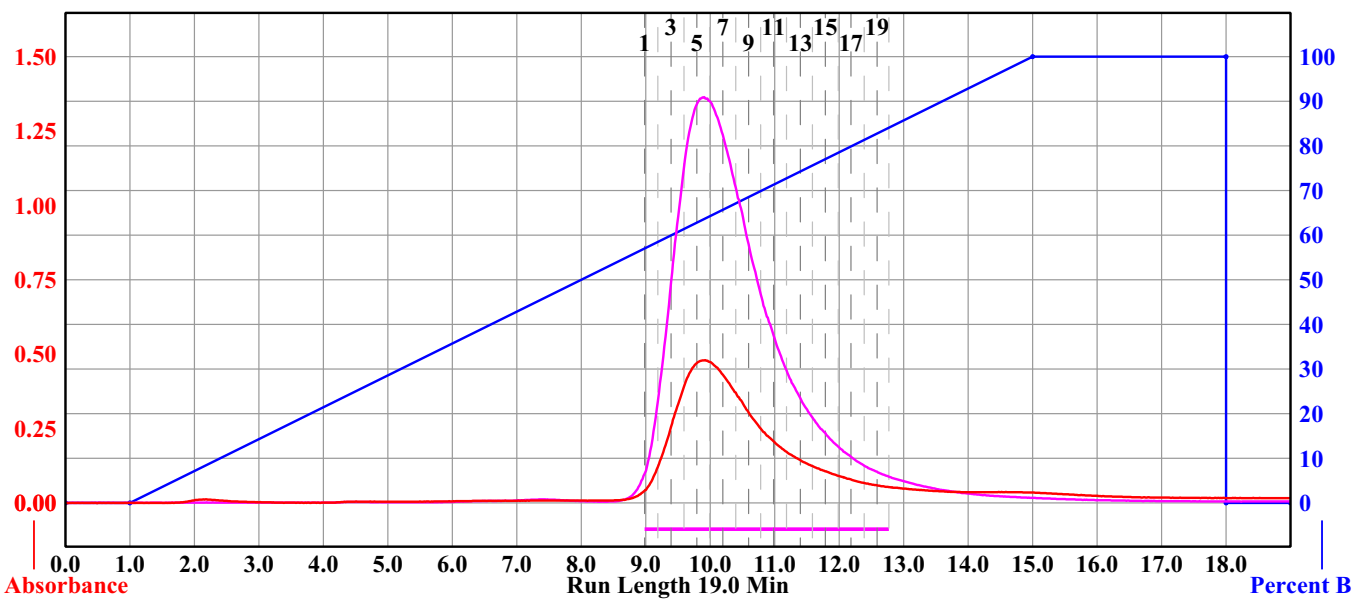

Rack A

\begin{tabular}{|c|c|c|c|c|c|}
\hline 108 & (107) & 106 & 105 & (104 & $1(13)$ \\
\hline 90 & $\mathscr{B}$ & V9 & (10) & (1) & $1(102$ \\
\hline ஜூ & (2) & $\mathscr{\theta}$ & 93 & (2) & (21) \\
\hline 85 & 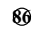 & 87 & 88 & 89 & (1) \\
\hline 8 & 83 & 82 & (11) & (81) & (19) \\
\hline 13 & (2) & (2) & (2) & (12) & 18 \\
\hline (12) & (11) & (11) & (6) & 6 & 60 \\
\hline (11) & (2) & 63 & 8 & (1) & ø \\
\hline (11) & 69 & 68 & 57 & ש6 & 55 \\
\hline (99) & (10) & (5) & (62) & 63 & 89 \\
\hline 48 & (47) & (46) & (45) & (4) & (4) \\
\hline (17) & B8 & (39) & (41) & (41) & (42) \\
\hline BO & (3) & B9 & B3 & (12) & (11) \\
\hline (2) & (6) & (27) & 68 & 29 & (31) \\
\hline 29 & 23 & (2) & (11) & (21) & (1) \\
\hline 0 & $\theta$ & (1) & (1) & 0 & 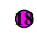 \\
\hline (1) & (1) & (1) & (1) & (8) & (1) \\
\hline (1) & (2) & (3) & (4) & (5) & (6) \\
\hline
\end{tabular}

$13 \mathrm{~mm}$ x $100 \mathrm{~mm}$ Tubes

\begin{tabular}{|c|c|c|c|}
\hline \multicolumn{2}{|c|}{ Peak \# } & Start Tube & End Tube \\
\hline \multicolumn{2}{|l|}{1} & \multirow[b]{2}{*}{ Solvent A } & \multirow[t]{2}{*}{ A:19 } \\
\hline Duration & $\%$ B & & \\
\hline 0.0 & 0.0 & A2 hexane & B2 ethyl acetate \\
\hline 1.0 & 0.0 & A2 hexane & B2 ethyl acetate \\
\hline 14.0 & 100.0 & A2 hexane & B2 ethyl acetate \\
\hline 3.0 & 100.0 & A2 hexane & B2 ethyl acetate \\
\hline 0.0 & 0.0 & A2 hexane & B2 ethyl acetate \\
\hline 1.0 & 0.0 & A2 hexane & B2 ethyl acetate \\
\hline & & & \\
\hline & & & \\
\hline & & & \\
\hline
\end{tabular}

Page 1 of 1 


\section{Synthetic Methods}

\subsubsection{4 (5-((4-fluorophenyl)amino)-2-(hexyloxy)phenyl)(pyrrolidin-1-} yl)methanone: G5-113s5

In a $20 \mathrm{~mL}$ vial with a septum cap and equipped with a drying tube open to the air, (5-amino-2-(hexyloxy)phenyl)(pyrrolidin-1-yl)methanone (G5-106s7, 1.06 mmol, $0.3065 \mathrm{~g})$, 4-fluorophenylboronic acid (1.5 mmol, $0.2128 \mathrm{~g}), \mathrm{Cu}(\mathrm{OAc})_{2}$ (2.3 mmol, $0.313 \mathrm{~g})$, triethylamine $(7.5 \mathrm{mmol}, 0.759 \mathrm{~g}, 1.045 \mathrm{~mL})$, powdered vacuum oven dried 4 Å molecular sieves (1 g) and anhydrous dichloromethane (DCM, $10 \mathrm{~mL}$ ) were combined. After stirring for 4 days, the reaction mixture was filtered through a cotton ball packed into a $10 \mathrm{~mL}$ syringe body, and washed with DCM. Silica gel ( $\sim 5 \mathrm{~g})$ was added to the filtrate, and the resulting mixture was concentrated by rotatory evaporation to a powder, which was used as a dry-load for silica chromatography (FIGURE 7.10). Fractions 23-28 were combined and concentrated by rotatory evaporation to an oil that later crystallized $(0.1578 \mathrm{~g}, 38.7 \%$ yield $)$.

Notes: In climates drier than Portland, Oregon, $0.3 \mathrm{~g}$ of powdered molecular sieves should suffice. Also, if one were to repeat this experiment using $1 \mathrm{~g}$ of powdered molecular sieves, increasing the amount of DCM used would improve the stirrability of the reaction.

\footnotetext{
${ }^{1}$ H-NMR $\delta(p p m)\left(\right.$ DMSO-d $\left._{6}\right): 7.87(1 \mathrm{H}, \mathrm{s}), 6.92-7.08(6 \mathrm{H}, \mathrm{m}), 6.81(1 \mathrm{H}, \mathrm{d}, \mathrm{J}$ $=2.69 \mathrm{~Hz}), 3.93(2 \mathrm{H}, \mathrm{t}, \mathrm{J}=6.22 \mathrm{~Hz}), 3.41(2 \mathrm{H}, \mathrm{t}, \mathrm{J}=6.80 \mathrm{~Hz}), 3.12-3.19(2 \mathrm{H}$, m), 1.74-1.90 (4 H, m), 1.60-1.69 (2 H, m), 1.33-1.44 (2 H, m), 1.26-1.33 (4 H, m), $0.88(3 \mathrm{H}, \mathrm{t}, \mathrm{J}=6.52 \mathrm{~Hz})$.
} 


\section{Synthetic Methods}

FIGURE 7.10: Chromatographic purification of G5-113s4.

Sample: grandy5-113s4

RediSep Column: Silica 40g

Flow Rate: $40 \mathrm{ml} / \mathrm{min}$

Equilibration Volume: $240.0 \mathrm{ml}$

Initial Waste: $0.0 \mathrm{ml}$

Air Purge: $1.0 \mathrm{~min}$

Solvent: A2 hexane

Solvent: B2 ethyl acetate
Rf 200 : OHSU COHEN RF200\#1

Peak Tube Volume: Max.

Non-Peak Tube Volume: Max.

Loading Type: Solid

Wavelength 1 (red): 254nm

Peak Width: 2 min

Threshold: 0.05 AU

Wavelength 2 (purple): 280nm
Monday 08 October 2012 01:45PM

Run Notes: self-packed column

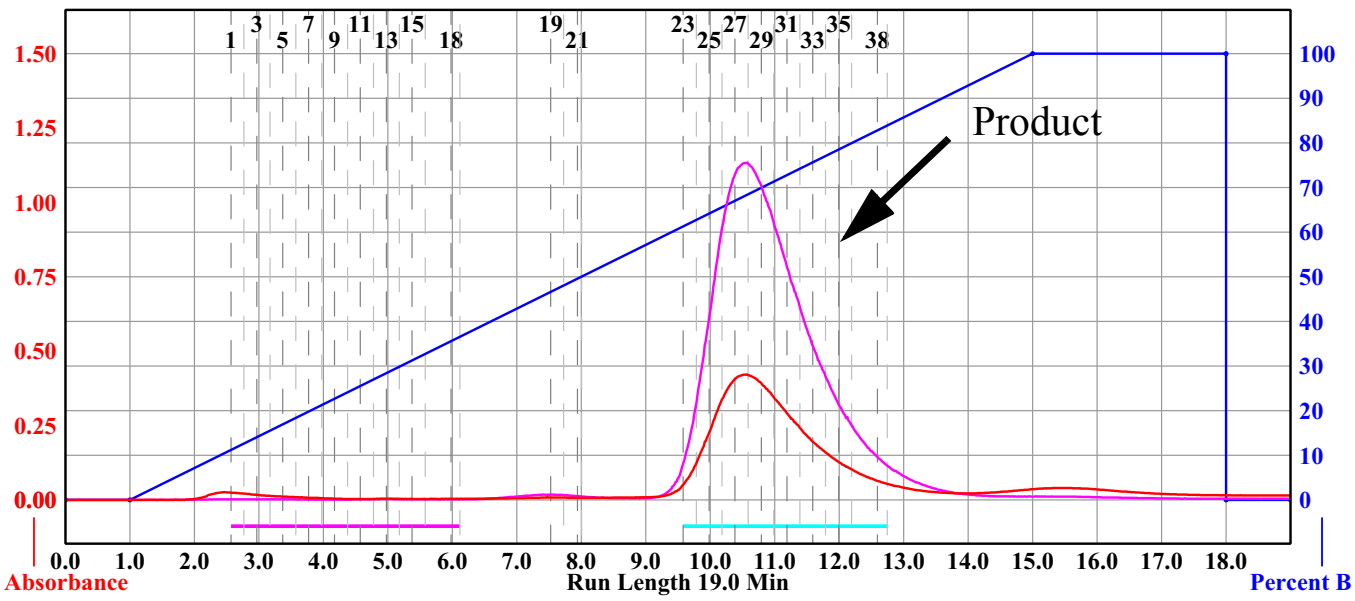

Rack A

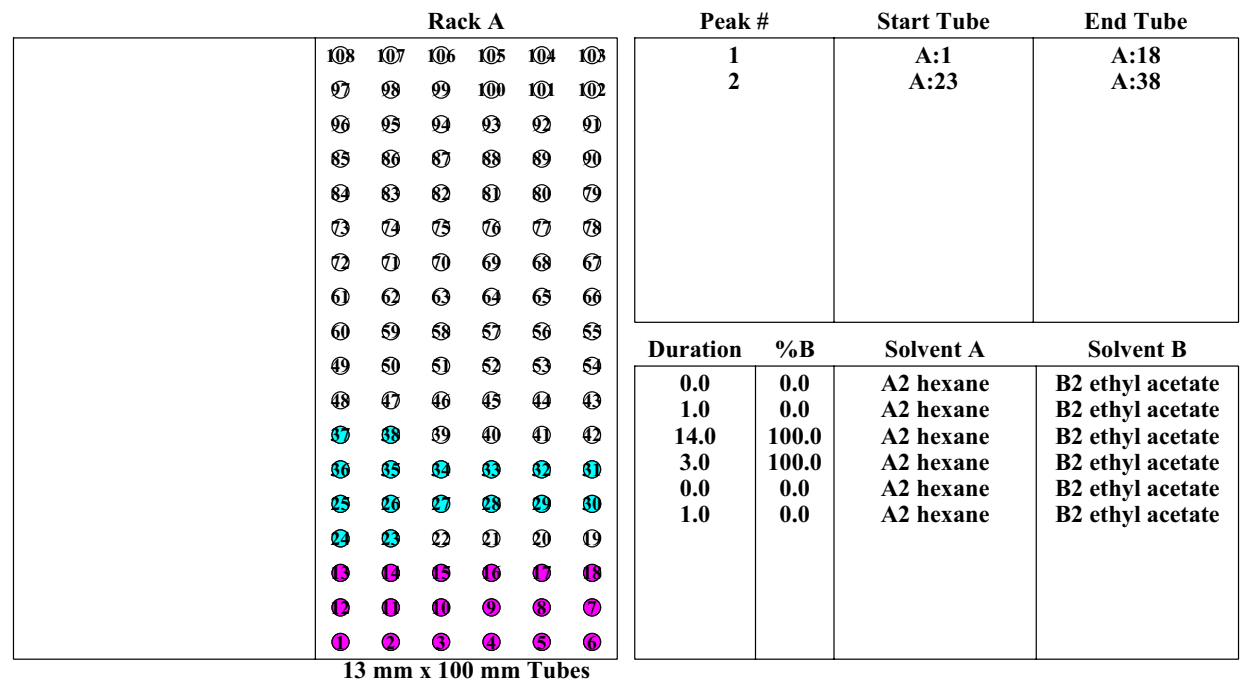

Page 1 of 1 


\section{Synthetic Methods}

\subsubsection{5 (5-((3-fluorophenyl)amino)-2-(hexyloxy)phenyl)(pyrrolidin-1-}

yl)methanone: G5-114s5

In a $20 \mathrm{~mL}$ vial with a septum cap and equipped with a drying tube open to the air, (5-amino-2-(hexyloxy)phenyl)(pyrrolidin-1-yl)methanone (G5-106s7, 0.83 mmol, $0.2406 \mathrm{~g})$, 3-fluorophenylboronic acid (1.5 mmol, $0.1396 \mathrm{~g}), \mathrm{Cu}(\mathrm{OAc})_{2}$ (2.3 mmol, $0.313 \mathrm{~g})$, triethylamine $(7.5 \mathrm{mmol}, 0.759 \mathrm{~g}, 1.045 \mathrm{~mL})$, powdered vacuum oven dried 4 Å molecular sieves (1 g) and anhydrous dichloromethane (DCM, $10 \mathrm{~mL}$ ) were combined. After stirring for 4 days, the reaction mixture was filtered through a cotton ball packed into a $10 \mathrm{~mL}$ syringe body, and washed with DCM. Silica gel ( $\sim 5 \mathrm{~g})$ was added to the filtrate, and the resulting mixture was concentrated by rotatory evaporation to a powder, which was used as a dry-load for silica chromatography (FIGURE 7.11). Fractions 1-15 were combined and concentrated by rotatory evaporation to an oil that later crystallized $(0.1897 \mathrm{~g}, 59.4 \%$ yield $)$.

Notes: In climates drier than Portland, Oregon, $0.3 \mathrm{~g}$ of powdered molecular sieves should suffice. Also, if one were to repeat this experiment using $1 \mathrm{~g}$ of powdered molecular sieves, increasing the amount of DCM used would improve the stirrability of the reaction.

${ }^{1} \mathrm{H}-\mathrm{NMR} \delta(\mathrm{ppm})\left(\mathrm{DMSO}_{6}\right): 8.19(1 \mathrm{H}, \mathrm{s}), 7.15-7.23(1 \mathrm{H}, \mathrm{m}), 7.12(1 \mathrm{H}, \mathrm{dd}$, $\mathrm{J}=8.80,2.72 \mathrm{~Hz}), 7.02(1 \mathrm{H}, \mathrm{d}, \mathrm{J}=8.83 \mathrm{~Hz}), 6.90(1 \mathrm{H}, \mathrm{d}, \mathrm{J}=2.74 \mathrm{~Hz}), 6.70-6.74$ (1 H, m), $6.63(1 \mathrm{H}, \mathrm{dt}, \mathrm{J}=12.01,2.29 \mathrm{~Hz}), 6.46-6.54(1 \mathrm{H}, \mathrm{m}), 3.96(2 \mathrm{H}, \mathrm{t}, \mathrm{J}=$ $6.23 \mathrm{~Hz}), 3.42(2 \mathrm{H}, \mathrm{t}, \mathrm{J}=6.67 \mathrm{~Hz}), 3.12-3.20(2 \mathrm{H}, \mathrm{m}), 1.75-1.89(4 \mathrm{H}, \mathrm{m}), 1.61-$ $1.71(2 \mathrm{H}, \mathrm{m}), 1.34-1.44(2 \mathrm{H}, \mathrm{m}), 1.26-1.34(4 \mathrm{H}, \mathrm{m}), 0.88(3 \mathrm{H}, \mathrm{t}, \mathrm{J}=6.68 \mathrm{~Hz})$. 


\section{Synthetic Methods}

FIGURE 7.11: Chromatographic purification of G5-114s4.

Sample: grandy5-114s4

RediSep Column: Silica 40g

Flow Rate: $40 \mathrm{ml} / \mathrm{min}$

Equilibration Volume: $240.0 \mathrm{ml}$

Initial Waste: $0.0 \mathrm{ml}$

Air Purge: $1.0 \mathrm{~min}$

Solvent: A2 hexane

Solvent: B2 ethyl acetate
Rf 200 : OHSU COHEN RF200\#1

Tuesday 09 October 2012 02:37PM

Peak Tube Volume: Max.

Non-Peak Tube Volume: Max.

Loading Type: Solid (Pause)

Wavelength 1 (red): 254nm

Peak Width: 2 min

Threshold: 0.05 AU

Wavelength 2 (purple): 280nm

Run Notes:

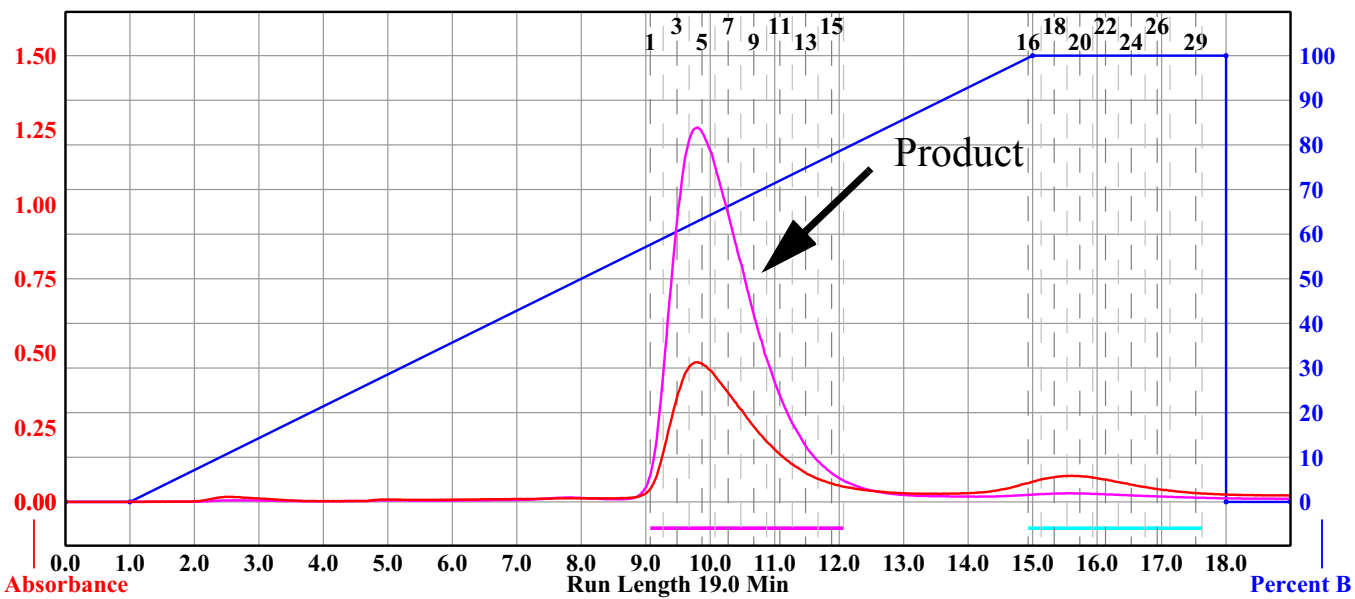

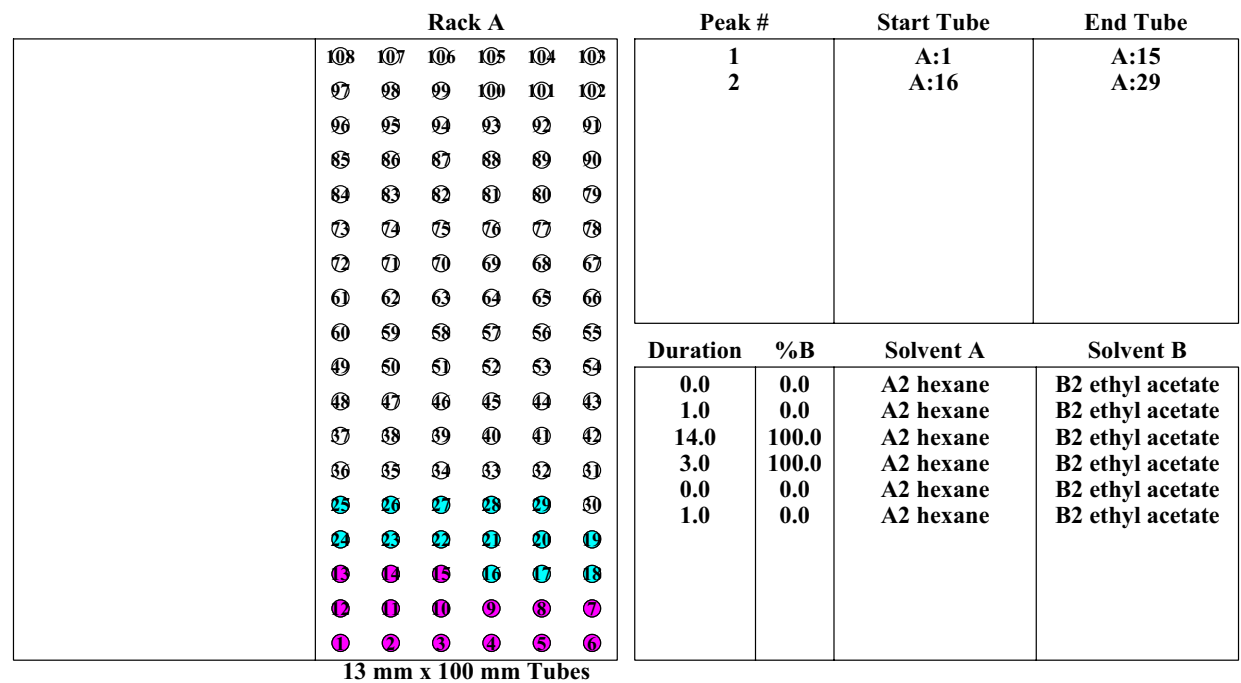

Page 1 of 1 


\section{Synthetic Methods}

\subsubsection{3-(1,3-dioxolan-2-yl)-4-(hexyloxy)- $N$-phenylaniline: G5-125s6}

In a $20 \mathrm{~mL}$ vial with a septum cap and equipped with a drying tube open to the air, 3-(1,3-dioxolan-2-yl)-4-(hexyloxy)aniline (G5-124s5, 2.2 mmol, 0.582 g), phenylboronic acid (3.3 mmol, $0.4024 \mathrm{~g}), \mathrm{Cu}(\mathrm{OAc})_{2}(4.8 \mathrm{mmol}, 0.8718 \mathrm{~g})$, triethylamine (16.5 mmol, $1.670 \mathrm{~g}, 2.3 \mathrm{~mL}$ ), powdered vacuum oven dried $4 \AA$ molecular sieves $(1 \mathrm{~g})$ and anhydrous dichloromethane (DCM, $10 \mathrm{~mL})$ were combined. After stirring for 3 days, the reaction mixture was slurried with 1:3 mixture of silica gel and celite ( $\sim 5 \mathrm{~g}$ total), and DCM. The slurry was filtered through a sintered glass funnel, and washed with DCM. Silica gel ( $\sim 5 \mathrm{~g})$ was added to the filtrate, and the resulting mixture was concentrated by rotatory evaporation to a powder, which was used as a dry-load for silica chromatography (FIGURE 7.12). Fractions 17-37 were combined and concentrated by rotatory evaporation to an oil that later crystallized $(0.3039 \mathrm{~g}, 37.7 \%$ yield $)$.

Notes: In climates drier than Portland, Oregon, $0.3 \mathrm{~g}$ of powdered molecular sieves should suffice. Also, if one were to repeat this experiment using $1 \mathrm{~g}$ of powdered molecular sieves, increasing the amount of DCM used would improve the stirrability of the reaction.

${ }^{1} \mathrm{H}-\mathrm{NMR} \delta(\mathrm{ppm})\left(\mathrm{DMSO}_{6}\right): 7.89(1 \mathrm{H}, \mathrm{s}), 7.13-7.21(3 \mathrm{H}, \mathrm{m}), 7.08(1 \mathrm{H}, \mathrm{dd}$, $\mathrm{J}=8.74,2.86 \mathrm{~Hz}), 6.94(1 \mathrm{H}, \mathrm{d}, \mathrm{J}=8.80 \mathrm{~Hz}), 6.88-6.93(2 \mathrm{H}, \mathrm{m}), 6.69-6.75(1 \mathrm{H}$, m), $5.96(1 \mathrm{H}, \mathrm{s}), 4.00-4.04(2 \mathrm{H}, \mathrm{m}), 3.88-3.99$ (4 H, m), 1.65-1.75 (2 H, m), 1.38$1.48(2 \mathrm{H}, \mathrm{m}), 1.28-1.35(4 \mathrm{H}, \mathrm{m}), 0.89(3 \mathrm{H}, \mathrm{t}, \mathrm{J}=6.77 \mathrm{~Hz})$. 


\section{Synthetic Methods}

${ }^{13} \mathrm{C}-\mathrm{NMR} \delta(\mathrm{ppm})\left(\right.$ DMSO-d $\left._{6}\right): 151.3,144.9,135.9,129.1,126.6,120.4$, $118.4,117.9,114.9,113.5,98.1,68.5,64.6,30.9,28.7,25.1,22.1,13.9$. 


\section{Synthetic Methods}

FIGURE 7.12: Chromatographic purification of G5-125s5.

Sample: grandy5-125s5

RediSep Column: Silica 40g

Flow Rate: $40 \mathrm{ml} / \mathrm{min}$

Equilibration Volume: 5.0 CV

Initial Waste: $0.0 \mathrm{CV}$

Air Purge: $1.0 \mathrm{~min}$

Solvent: A2 hexane

Solvent: B2 ethyl acetate
Rf 200 : OHSU COHEN RF200\#1

Peak Tube Volume: Max.

Non-Peak Tube Volume: Max.

Loading Type: Solid

Wavelength 1 (red): 254nm

Peak Width: 2 min

Threshold: 0.05 AU

Wavelength 2 (purple): 280nm
Tuesday 23 October 2012 11:15AM

All Wavelength (orange): 200nm - 360nm

Peak Width: 2 min

Threshold: 0.20 AU

Run Notes: self-packed column

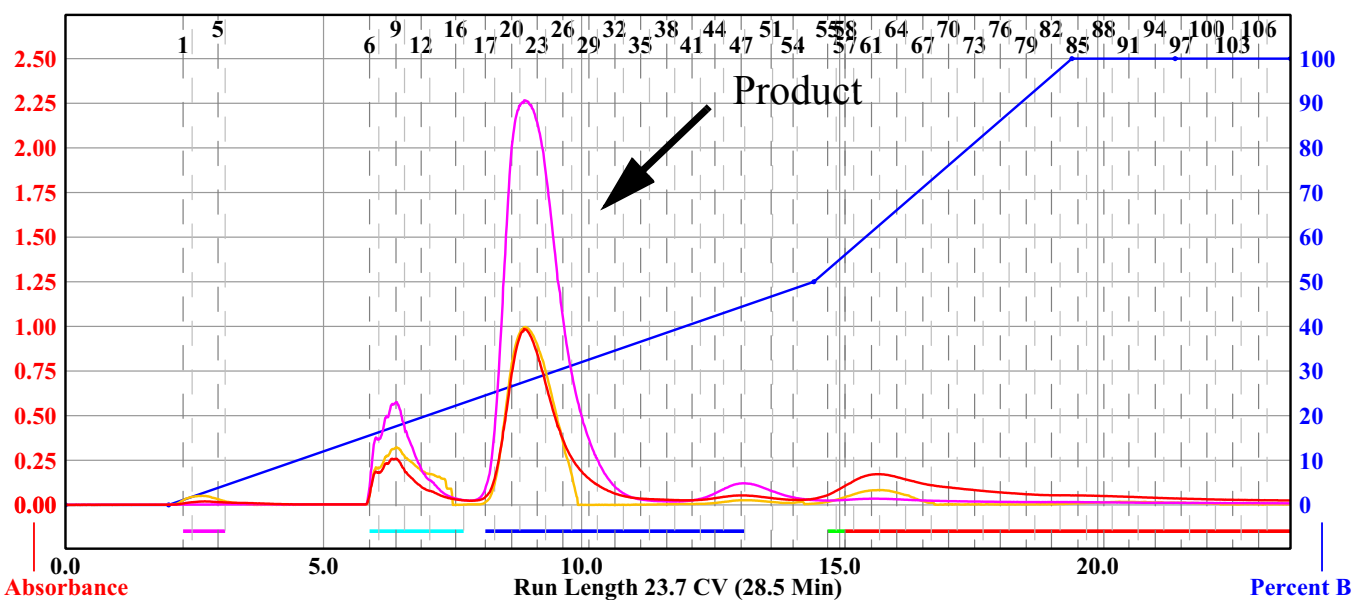

Rack A

Rack B

\begin{tabular}{|c|c|c|c|c|c|c|c|c|c|c|c|}
\hline 108 & 107 & 106 & 105 & 104 & 103 & 108 & (10) & 106 & 105 & 1(144 & (103 \\
\hline 8 & 28 & 0 & 100 & 10 & 102 & 97 & 8 & $\mathscr{1 9}$ & 100 & (101 & (102 \\
\hline 26 & (2) & (2) & 2 & 0 & (D) & ஜ & (13 & (4) & (2) & (2) & (21) \\
\hline 35 & 8 & 87 & 8 & 8 & ம & 85 & 80 & 87 & 88 & 89 & ஜD \\
\hline (3) & 8 & (2) & (1) & (1) & 0 & 8 & 83 & 82 & (11) & 80 & 0 \\
\hline 13 & 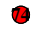 & (2) & (6) & 0 & 0 & 13 & 84 & (1) & (10) & (17) & 88 \\
\hline (2) & 0 & (1) & (9) & 6 & 0 & (12) & (11) & (10) & 69 & 6 & 60 \\
\hline (1) & (2) & 6 & $\theta$ & (6) & (1) & (1) & (2) & 63 & 9 & 6 & 60 \\
\hline (1) & (9) & 68 & 57 & ஏ0 & 65 & (1) & 69 & 68 & 57 & 60 & 65 \\
\hline 49 & b0 & (1) & (2) & 33 & 69 & (49) & פ0 & (1) & (2) & 63 & 69 \\
\hline 48 & 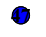 & (1) & C & 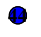 & 0 & (48) & (47) & (46) & 45 & (4) & (43) \\
\hline & 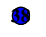 & 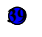 & (1) & 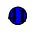 & 0 & (B) & B8 & B9 & (41) & (41) & (42) \\
\hline & 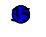 & 0 & 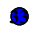 & 0 & - & BV & BS & B9 & B3 & (32) & (BD) \\
\hline & ก & $\Omega$ & (2) & 0 & - & (2) & ש6 & (27) & 8 & 29 & (10) \\
\hline 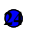 & - & 0 & 0 & • & 0 & (29) & 23 & (22) & (21) & (21) & (19) \\
\hline (1) & $\mathbb{B}$ & (1) & (10) & 0 & - & (1) & (1) & (1) & (1) & (1) & 18 \\
\hline (12) & (1) & (1) & (9) & (8) & (1) & (12) & (11) & (1) & (9) & (8) & (7) \\
\hline (1) & (2) & (3) & (4) & (5) & (6) & (1) & (2) & (3) & (4) & (5) & (6) \\
\hline
\end{tabular}

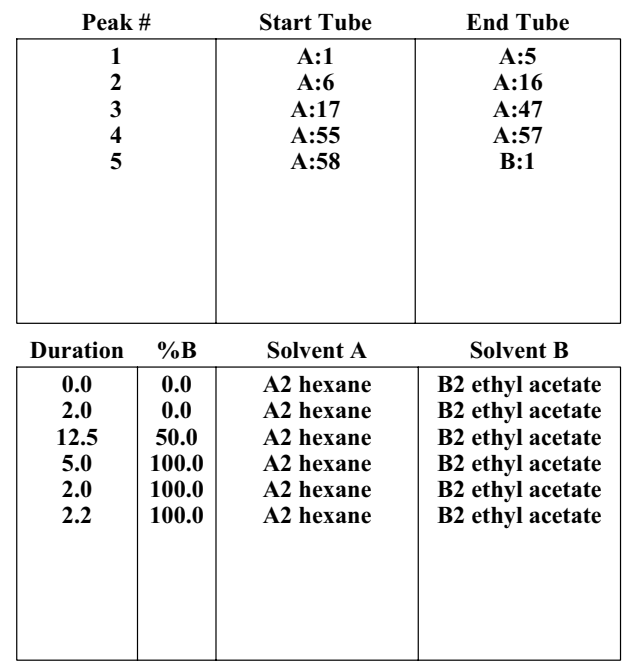

Page 1 of 1 


\section{Synthetic Methods}

\subsubsection{3-(1,3-dioxolan-2-yl)- $N$-(4-fluorophenyl)-4-(hexyloxy)aniline: G5-126s6}

In a $20 \mathrm{~mL}$ vial with a septum cap and equipped with a drying tube open to the air, 3-(1,3-dioxolan-2-yl)-4-(hexyloxy)aniline (G5-124s5, $2.66 \mathrm{mmol}, 0.7052 \mathrm{~g})$, 4-fluorophenylboronic acid (4.05 mmol, $0.5667 \mathrm{~g}), \mathrm{Cu}(\mathrm{OAc})_{2}$ (6 mmol, $\left.1.090 \mathrm{~g}\right)$, triethylamine (20.25 mmol, $2.049 \mathrm{~g}, 2.822 \mathrm{~mL})$, powdered vacuum oven dried $4 \AA$ molecular sieves ( $1 \mathrm{~g})$ and anhydrous dichloromethane (DCM, $10 \mathrm{~mL})$ were combined. After stirring for 3 days, the reaction mixture was slurried with 1:3 mixture of silica gel and celite ( $\sim 5 \mathrm{~g}$ total), and DCM. The slurry was filtered through a sintered glass funnel, and washed with DCM. Silica gel ( $\sim 5 \mathrm{~g})$ was added to the filtrate, and the resulting mixture was concentrated by rotatory evaporation to a powder, which was used as a dry-load for silica chromatography (FIGURE 7.13). Fractions 22-37 were combined and concentrated by rotatory evaporation to an oil that later crystallized $(0.3211 \mathrm{~g}, 32.3 \%$ yield $)$.

Notes: In climates drier than Portland, Oregon, $0.3 \mathrm{~g}$ of powdered molecular sieves should suffice. Also, if one were to repeat this experiment using $1 \mathrm{~g}$ of powdered molecular sieves, increasing the amount of DCM used would improve the stirrability of the reaction.

${ }^{1} \mathrm{H}-\mathrm{NMR} \delta(\mathrm{ppm})\left(\right.$ DMSO-d $\left._{6}\right): 7.84(1 \mathrm{H}, \mathrm{s}), 7.12(1 \mathrm{H}, \mathrm{d}, \mathrm{J}=2.84 \mathrm{~Hz}), 6.99$ -

7.06 (3 H, m), 6.88-6.95 (3 H, m), 5.95 (1 H, s), 4.00-4.04 (2 H, m), 3.90-3.96 (4 H, m), 1.65-1.73 (2 H, m), 1.37-1.48 (2 H, m), 1.28-1.35 (4 H, m), 0.85-0.92 (3 H, m). 


\section{Synthetic Methods}

FIGURE 7.13: Chromatographic purification of G5-126s5.

Sample: grandy5-126s

RediSep Column: Silica 40g

Flow Rate: $\mathbf{4 0}$ ml/min

Equilibration Volume: 5.0 CV

Initial Waste: $0.0 \mathrm{CV}$

Air Purge: $1.0 \mathrm{~min}$

Solvent: A2 hexane

Solvent: B2 ethyl acetate
Rf 200 : OHSU COHEN RF200\#1

Peak Tube Volume: Max.

Non-Peak Tube Volume: Max.

Loading Type: Solid

Wavelength 1 (red): 254nm

Peak Width: 2 min

Threshold: $0.05 \mathrm{AU}$

Wavelength 2 (purple): 280nm
Wednesday 24 October 2012 01:21PM

All Wavelength (orange): 200nm - 360nm

Peak Width: 2 min

Threshold: 0.20 AU

Run Notes:

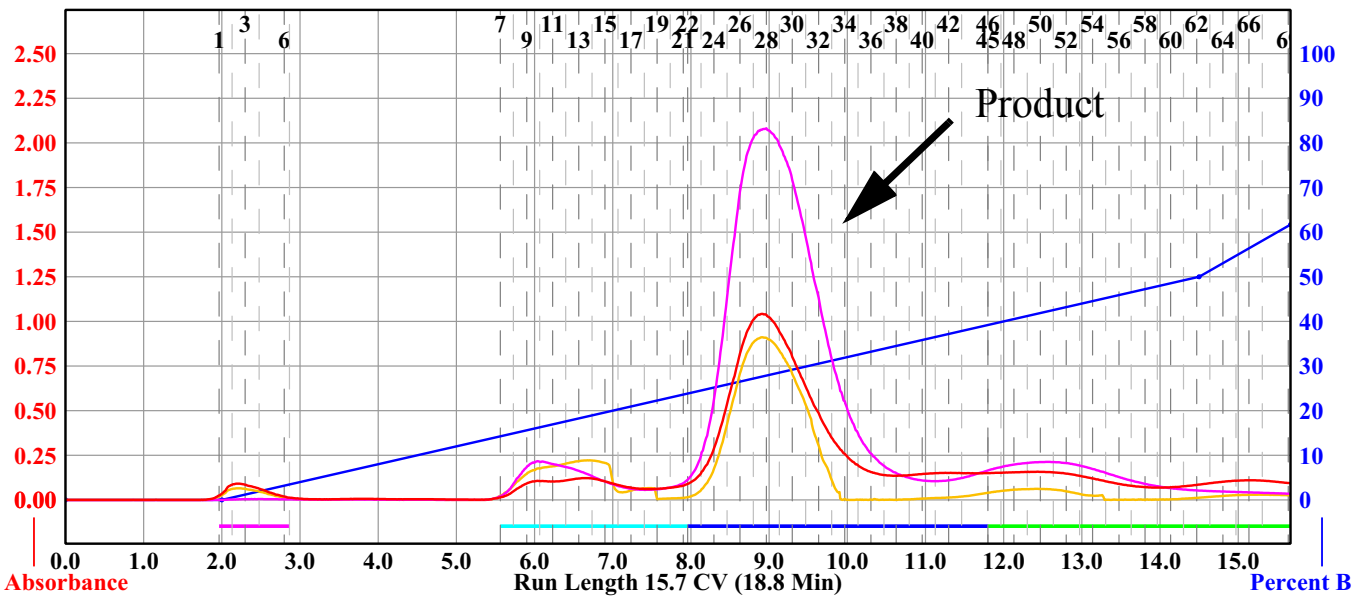

Rack A

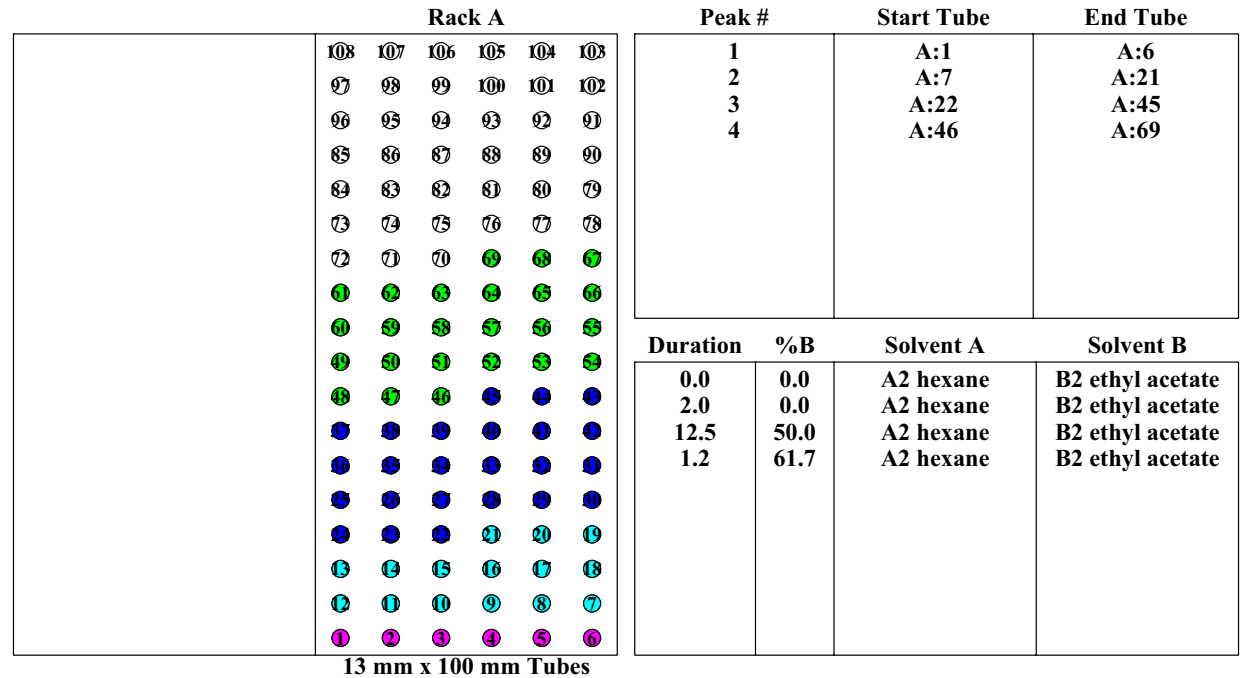

Page 1 of 1 


\section{Synthetic Methods}

\subsubsection{3-(1,3-dioxolan-2-yl)- $N$-(3-fluorophenyl)-4-(hexyloxy)aniline: G5-127s6}

In a $20 \mathrm{~mL}$ vial with a septum cap and equipped with a drying tube open to the air, 3-(1,3-dioxolan-2-yl)-4-(hexyloxy)aniline (G5-124s5, 2.64 mmol, $0.7011 \mathrm{~g})$, 3-fluorophenylboronic acid (4.05 mmol, $0.5667 \mathrm{~g}), \mathrm{Cu}(\mathrm{OAc})_{2}$ (6 mmol, $\left.1.090 \mathrm{~g}\right)$, triethylamine $(20.25 \mathrm{mmol}, 2.049 \mathrm{~g}, 2.822 \mathrm{~mL})$, powdered vacuum oven dried $4 \AA$ molecular sieves ( $1 \mathrm{~g})$ and anhydrous dichloromethane (DCM, $10 \mathrm{~mL}$ ) were combined. After stirring for 3 days, the reaction mixture was slurried with 1:3 mixture of silica gel and celite ( $\sim 5 \mathrm{~g}$ total), and DCM. The slurry was filtered through a sintered glass funnel, and washed with DCM. Silica gel ( $\sim 5 \mathrm{~g})$ was added to the filtrate, and the resulting mixture was concentrated by rotatory evaporation to a powder, which was used as a dry-load for silica chromatography (FIGURE 7.14). Fractions 22-37 were combined and concentrated by rotatory evaporation to an oil that later crystallized ( $0.4405 \mathrm{~g}, 44.7 \%$ yield $)$.

Notes: In climates drier than Portland, Oregon, $0.3 \mathrm{~g}$ of powdered molecular sieves should suffice. Also, if one were to repeat this experiment using $1 \mathrm{~g}$ of powdered molecular sieves, increasing the amount of DCM used would improve the stirrability of the reaction.

\footnotetext{
${ }^{1} \mathrm{H}-\mathrm{NMR} \delta(\mathrm{ppm})\left(\mathrm{DMSO}_{6}\right): 8.17(1 \mathrm{H}, \mathrm{s}), 7.10-7.21(3 \mathrm{H}, \mathrm{m}), 6.98(1 \mathrm{H}, \mathrm{d}, \mathrm{J}$ = 8.75 Hz), 6.66-6.71 (1 H, m), $6.59(1 \mathrm{H}, \mathrm{dt}, \mathrm{J}=12.09,2.30 \mathrm{~Hz}), 6.44-6.51(1 \mathrm{H}$, m), 5.97 (1 H, s), 3.89-4.04 (6 H, m), 1.66-1.75 (2 H, m), 1.38-1.49 (2 H, m), 1.31$1.32(4 \mathrm{H}, \mathrm{m}), 0.89(3 \mathrm{H}, \mathrm{t}, \mathrm{J}=6.74 \mathrm{~Hz})$.
} 


\section{Synthetic Methods}

FIGURE 7.14: Chromatographic purification of G5-127s5.

Sample: grandy5-127s5

RediSep Column: Silica 40g

Flow Rate: $40 \mathrm{ml} / \mathrm{min}$

Equilibration Volume: 5.0 CV

Initial Waste: $0.0 \mathrm{CV}$

Air Purge: $1.0 \mathrm{~min}$

Solvent: A2 hexane

Solvent: B2 ethyl acetate
Rf 200 : OHSU COHEN RF200\#1

Peak Tube Volume: Max.

Non-Peak Tube Volume: Max.

Loading Type: Solid

Wavelength 1 (red): 254nm

Peak Width: 2 min

Threshold: 0.05 AU

Wavelength 2 (purple): 280nm
Wednesday 24 October 2012 01:52PM

All Wavelength (orange): $200 \mathrm{~nm}-360 \mathrm{~nm}$

Peak Width: 2 min

Threshold: 0.20 AU

Run Notes:

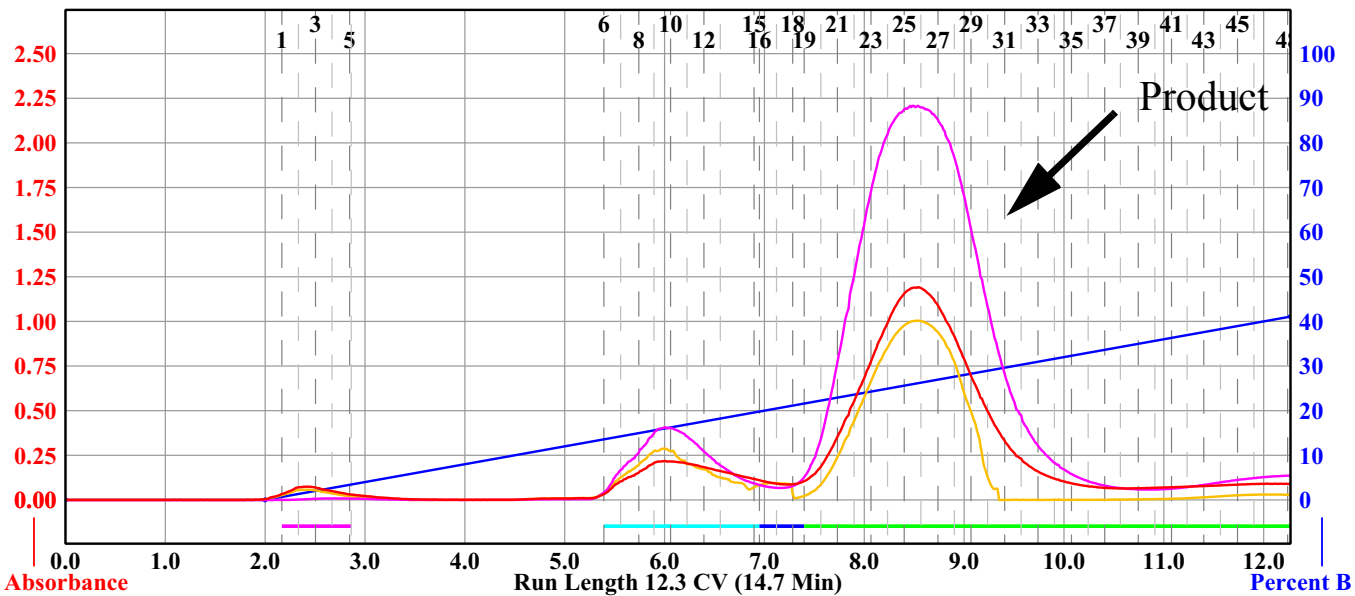

Rack A

\begin{tabular}{|c|c|c|c|c|c|}
\hline 108 & (107 & (106 & 105 & (104 & (103 \\
\hline 02 & $\mathscr{0}$ & $\mathscr{V}$ & (10) & (10) & (102 \\
\hline ש6 & (5) & அ & (2) & (2) & (21) \\
\hline 89 & 80 & 87 & $B$ & 89 & D10 \\
\hline 89 & 83 & 82 & (11) & (10) & (19) \\
\hline 13 & (2) & (2) & (20) & (2) & 88 \\
\hline (12) & (11) & (10) & 69 & 68 & (10) \\
\hline (11) & (2) & 63 & 9 & 67 & ஐ \\
\hline (10) & 59 & 68 & 67 & ש6 & 65 \\
\hline (49) & b0 & (51) & 62 & 63 & 69 \\
\hline (48) & (47) & (46) & (45) & (4) & (3) \\
\hline BD & 68 & (39) & (41) & (11) & (42) \\
\hline (16) & (3) & (3) & (3) & (2) & (11) \\
\hline (3) & (26) & 87 & 8 & 29 & (BD \\
\hline (4) & (23) & (2) & (21) & (21) & (1) \\
\hline (13) & (1) & (1) & 0 & 0 & 0 \\
\hline (12) & (11) & (11) & (9) & (8) & (1) \\
\hline (1) & (2) & (3) & (4) & (5) & (6) \\
\hline
\end{tabular}

$13 \mathrm{~mm} \times 100 \mathrm{~mm}$ Tubes

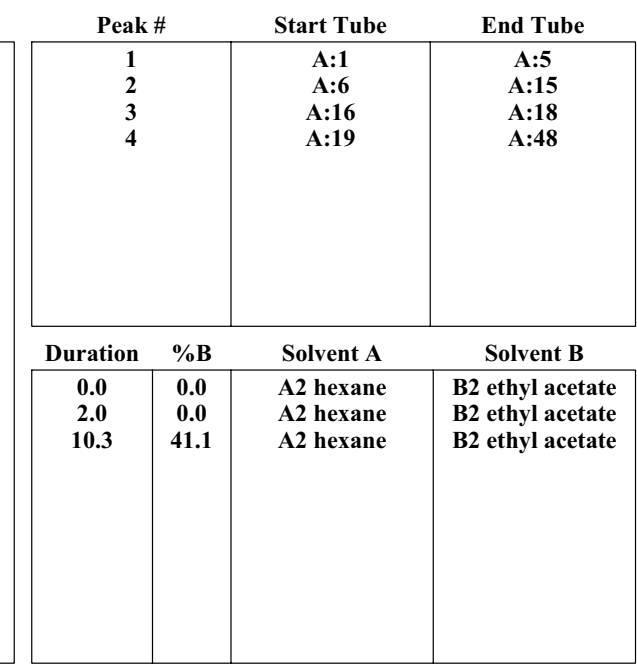

Page 1 of 1 


\section{Synthetic Methods}

\subsubsection{Hexyl 5-(benzylamino)-2-(hexyloxy)benzoate: G5-049s10}

In a $20 \mathrm{~mL}$ vial, hexyl 5-amino-2-(hexyloxy)benzoate (G5-047s9, 82.95

mass\% w/EtOAc, 3 mmol, $1.1669 \mathrm{~g}$ ), and benzaldehyde (3 mmol, $0.3184 \mathrm{~g}, 0.305$

$\mathrm{mL})$ were dissolved in methanol $(10 \mathrm{~mL})$. The solution was stirred for 15 minutes, then $\mathrm{NaBH}_{3} \mathrm{CN}$ (3.3 mmol, $0.2135 \mathrm{~g}$ ) was slowly added, and the reaction stirred for another 18 hours. The reaction was worked-up by adding $1 \mathrm{M} \mathrm{NaOH}(3 \mathrm{~mL})$, then extracting with hexanes $(2 \times 3 \mathrm{~mL})$. The combined hexane phases were washed with water $(3 \mathrm{~mL})$, then dried with $\mathrm{MgSO}_{4}$. After filtering, basic alumina $(\sim 2 \mathrm{~g})$ was added to the filtrate. The mixture was concentrated by rotatory evaporation to a powder, which was used as a dry-load for silica chromatography (FIG-

URE 7.15). Fractions $3-8$ were combined and concentrated by rotatory evaporation (0.6768 g, 54.7\% yield).

${ }^{1} \mathrm{H}-\mathrm{NMR} \delta(\mathrm{ppm})\left(\right.$ DMSO-d $\left._{6}\right):$ 7.28-7.37 (4 H, m), 7.19-7.25 (1 H, m), 6.82-

$6.89(2 \mathrm{H}, \mathrm{m}), 6.71(1 \mathrm{H}, \mathrm{dd}, \mathrm{J}=8.83,3.08 \mathrm{~Hz}), 6.10(1 \mathrm{H}, \mathrm{t}, \mathrm{J}=6.10 \mathrm{~Hz}), 4.23(2$ $\mathrm{H}, \mathrm{d}, \mathrm{J}=6.09 \mathrm{~Hz}), 4.15(2 \mathrm{H}, \mathrm{t}, \mathrm{J}=6.48 \mathrm{~Hz}), 3.84(2 \mathrm{H}, \mathrm{t}, \mathrm{J}=6.42 \mathrm{~Hz}), 1.58-1.68$ (4 H, m), 1.34-1.43 (4 H, m), 1.25-1.34 (8 H, m), 0.84-0.91 (6 H, m).

${ }^{13} \mathrm{C}-\mathrm{NMR} \delta(\mathrm{ppm})\left(\mathrm{DMSO}_{6}\right): 166.6,148.7,142.5,140.1,128.2,127.1$, $126.6,121.6,116.7,115.9,113.8,69.4,64.2,46.9,31.0,30.9,28.9,28.2,25.2(2$ C), 22.1, 22.0, 13.9 (2 C). 


\section{Synthetic Methods}

FIGURE 7.15: Chromatographic purification of G5-049s7.

Sample: grandy5-049s7

RediSep Column: Silica 24g

Flow Rate: 35 ml/min

Equilibration Volume: 5.0 CV

Initial Waste: $0.0 \mathrm{CV}$

Air Purge: $1.0 \mathrm{~min}$

Solvent: A2 hexane

Solvent: B2 ethyl acetate
Rf 200 : OHSU COHEN RF200\#1

Peak Tube Volume: Max.

Non-Peak Tube Volume: Max.

Loading Type: Solid (Pause)

Wavelength 1 (red): 254nm

Peak Width: 1 min

Threshold: $0.05 \mathrm{AU}$

Wavelength 2 (purple): 280nm
Thursday 16 August 2012 02:04PM

Run Notes:

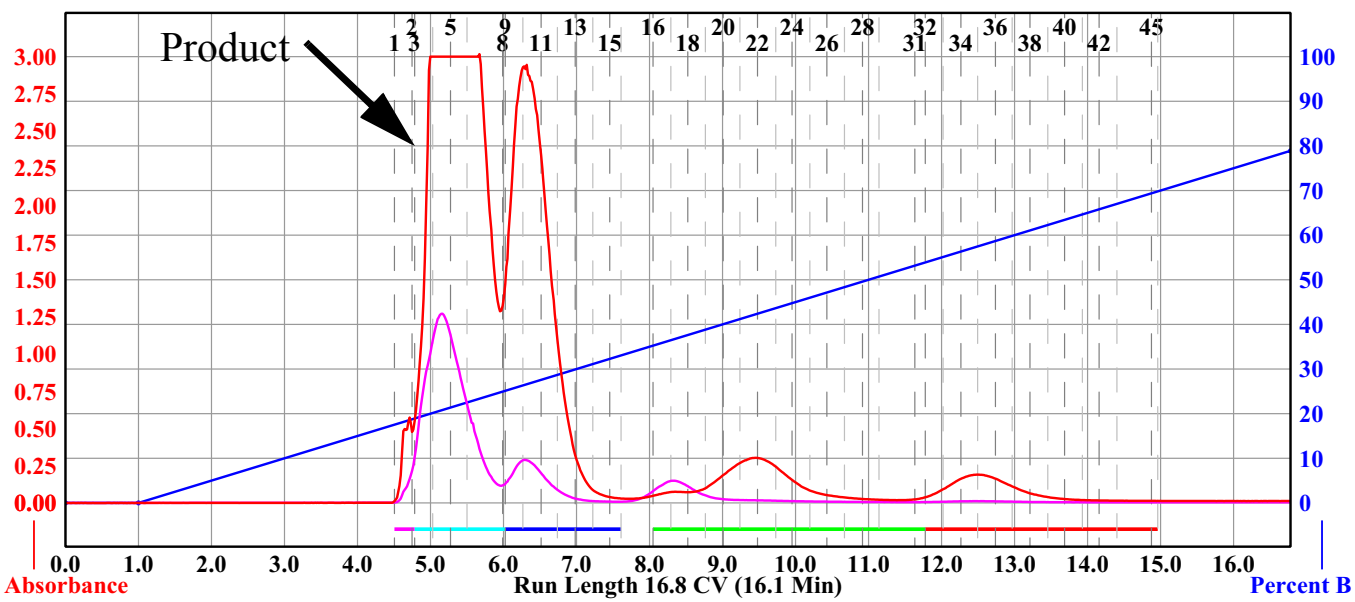

Rack A

\begin{tabular}{|c|c|c|c|c|c|}
\hline 108 & (107 & 106 & 105 & (144 & (103 \\
\hline (2) & $\mathscr{B}$ & (99 & (100) & (1) & (102 \\
\hline ஜ & ஏु & (2) & (23) & (22) & (21) \\
\hline 89 & 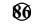 & 87 & 88 & 89 & (2) \\
\hline 8 & 83 & (2) & (11) & (10) & (1) \\
\hline 13 & (3) & (2) & (20) & (27) & 88 \\
\hline (12) & (11) & (10) & (9) & 68 & (6) \\
\hline (11) & (2) & 63 & 6 & 6 & ஞ \\
\hline (1) & 59 & 68 & (57) & 60 & 65 \\
\hline (49) & (10) & (1) & (2) & 63 & 69 \\
\hline 48 & (47) & (46) & (6) & 4 & (3) \\
\hline (3) & (2) & (2) & (1) & (1) & (1) \\
\hline (B) & (5) & (3) & (3) & (2) & (11) \\
\hline (2) & (6) & 07 & 28 & 29 & (10) \\
\hline 89 & 23 & (2) & (2) & (21) & (1) \\
\hline 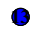 & 0 & 0 & (10) & 10 & 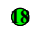 \\
\hline & 0 & 0 & - & (8) & (7) \\
\hline ( & (2) & (3) & (4) & (5) & (6) \\
\hline
\end{tabular}

$13 \mathrm{~mm} \times 100 \mathrm{~mm}$ Tubes

\begin{tabular}{|c|c|c|c|}
\hline \multicolumn{2}{|c|}{ Peak \# } & Start Tube & End Tube \\
\hline $\begin{array}{l}1 \\
2 \\
3 \\
4 \\
5\end{array}$ & & $\begin{array}{c}A: 1 \\
A: 3 \\
A: 9 \\
A: 16 \\
A: 32\end{array}$ & $\begin{array}{c}A: 2 \\
A: 8 \\
A: 15 \\
A: 31 \\
A: 45\end{array}$ \\
\hline Duration & $\%$ B & Solvent A & Solvent B \\
\hline $\begin{array}{c}0.0 \\
1.0 \\
15.8\end{array}$ & $\begin{array}{c}0.0 \\
0.0 \\
78.9\end{array}$ & $\begin{array}{l}\text { A2 hexane } \\
\text { A2 hexane } \\
\text { A2 hexane }\end{array}$ & $\begin{array}{l}\text { B2 ethyl acetate } \\
\text { B2 ethyl acetate } \\
\text { B2 ethyl acetate }\end{array}$ \\
\hline
\end{tabular}

Page 1 of 1 


\section{Synthetic Methods}

\subsubsection{Hexyl 2-(hexyloxy)-5-((4-fluorobenzyl)amino)benzoate: G5-050s10}

In a $20 \mathrm{~mL}$ vial, hexyl 5-amino-2-(hexyloxy)benzoate (G5-047s9, 82.95

mass\% w/EtOAc, 3 mmol, $1.1674 \mathrm{~g}$ ), and 4-fluorobenzaldehyde (3 mmol, 0.3723

$\mathrm{g}, 0.3218 \mathrm{~mL})$ were dissolved in methanol $(10 \mathrm{~mL})$. The solution was stirred for 15

minutes, then $\mathrm{NaBH}_{3} \mathrm{CN}(3.3 \mathrm{mmol}, 0.2101 \mathrm{~g})$ was slowly added, and the reaction

stirred for another 18 hours. The reaction was worked-up by adding $1 \mathrm{M} \mathrm{NaOH}$ (3

$\mathrm{mL})$, then extracting with hexanes $(2 \times 3 \mathrm{~mL})$. The combined hexane phases were washed with water $(3 \mathrm{~mL})$, then dried with $\mathrm{MgSO}_{4}$. After filtering, basic alumina $(\sim 2 \mathrm{~g})$ was added to the filtrate. The mixture was concentrated by rotatory evaporation to a powder, which was used as a dry-load for silica chromatography (FIG-

URE 7.16). Fractions $12-25$ were combined and concentrated by rotatory evaporation to an oil ( $0.6987 \mathrm{~g}, 54.2 \%$ yield).

${ }^{1} \mathrm{H}-\mathrm{NMR} \delta(\mathrm{ppm})\left(\mathrm{DMSO}_{6}\right):$ 7.34-7.40 (2 H, m), 7.10-7.17 (2 H, m), 6.86 (1 $\mathrm{H}, \mathrm{d}, \mathrm{J}=8.88 \mathrm{~Hz}), 6.83(1 \mathrm{H}, \mathrm{d}, \mathrm{J}=2.96 \mathrm{~Hz}), 6.70(1 \mathrm{H}, \mathrm{dd}, \mathrm{J}=8.92,3.00 \mathrm{~Hz})$, $6.11(1 \mathrm{H}, \mathrm{t}, \mathrm{J}=6.12 \mathrm{~Hz}), 4.21(2 \mathrm{H}, \mathrm{d}, \mathrm{J}=6.04 \mathrm{~Hz}), 4.15(2 \mathrm{H}, \mathrm{t}, \mathrm{J}=6.48 \mathrm{~Hz}), 3.84$ (2 H, t, J = 6.44 Hz), 1.58-1.68 (4 H, m), 1.33-1.43 (4 H, m), 1.23-1.33 (8 H, m), 084-0.90 (6 H, m).

${ }^{13} \mathrm{C}-\mathrm{NMR} \delta(\mathrm{ppm})\left(\mathrm{DMSO}-\mathrm{d}_{6}\right): 166.6,161.0(1 \mathrm{C}, \mathrm{d}, \mathrm{J}=241.76 \mathrm{~Hz}), 148.6(1$ $\mathrm{C}, \mathrm{d}, \mathrm{J}=44.51 \mathrm{~Hz}), 142.3,136.2(1 \mathrm{C}, \mathrm{d}, \mathrm{J}=2.89 \mathrm{~Hz}), 129.0(1 \mathrm{C}, \mathrm{d}, \mathrm{J}=8.03 \mathrm{~Hz})$, $121.6,116.7,115.9,115.0,114.8,113.9,69.3,64.2,46.2,31.0,30.9,28.9,28.2$, 25.2 (2 C), 22.1, 22.0, 13.9 (2 C). 


\section{Synthetic Methods}

FIGURE 7.16: Chromatographic purification of G5-050s7.

Sample: grndy5-050s7

RediSep Column: Silica 24g SN: E041039E8A1DA Lot: 1920177010W

Flow Rate: $35 \mathrm{ml} / \mathrm{min}$

Equilibration Volume: 5.0 CV

Initial Waste: $0.0 \mathrm{CV}$

Air Purge: $1.0 \mathrm{~min}$

Solvent: A2 hexane

Solvent: B2 ethyl acetate
Rf 200 : OHSU COHEN RF200\#1

Thursday 16 August 2012 03:51PM

Peak Tube Volume: Max.

Non-Peak Tube Volume: Max.

Loading Type: Solid

Wavelength 1 (red): 254nm

Peak Width: 1 min

Threshold: 0.05 AU

Wavelength 2 (purple): 280nm

Run Notes:

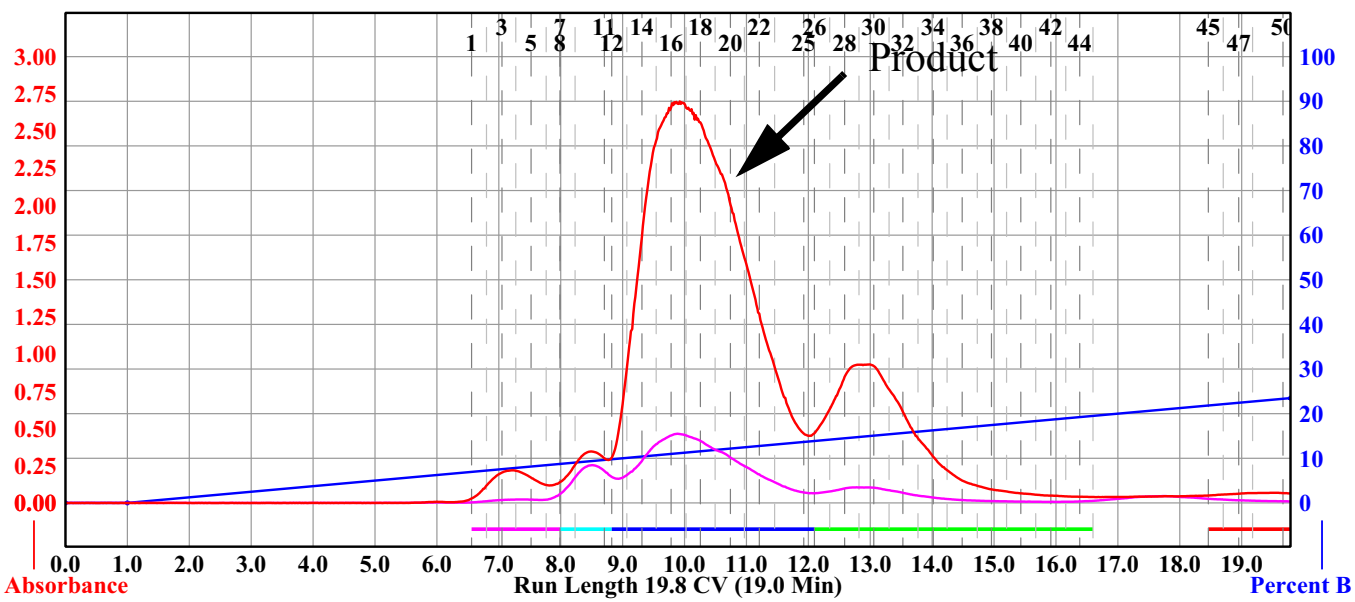

Rack A

\begin{tabular}{|c|c|c|c|c|c|}
\hline (108 & (107) & 106 & 105 & (144) & (103 \\
\hline (27) & 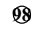 & (99 & (10) & (1) & (102 2 \\
\hline (6) & (25 & (2) & 93 & (2) & (21) \\
\hline 8 & 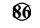 & 87 & 88 & 89 & (2) \\
\hline 8 & 8 & (2) & (11) & (10) & 10 \\
\hline$(3)$ & (3) & (2) & (2) & (27) & 88 \\
\hline (12) & (11) & (10) & (9) & 68 & (6) \\
\hline (1) & (2) & 63 & 9 & 67 & ஞ \\
\hline (1) & 59 & 68 & 57 & 60 & 69 \\
\hline (4) & (1) & (1) & 62 & 63 & 69 \\
\hline (4) & (6) & (6) & (65) & (4) & (4) \\
\hline (3) & B8 & (3) & (4) & (41) & (4) \\
\hline (16) & (3) & (3) & (3) & (32) & (11) \\
\hline d & (6) & 07 & 28 & 29 & (10) \\
\hline & 0 & 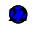 & 0 & & - \\
\hline & 0 & 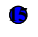 & 0 & 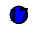 & - \\
\hline$C$ & (11) & (11) & (2) & (8) & (1) \\
\hline A & (2) & (3) & (4) & (5) & (6) \\
\hline
\end{tabular}

$13 \mathrm{~mm} \times 100 \mathrm{~mm}$ Tubes

\begin{tabular}{|c|c|c|c|}
\hline \multicolumn{2}{|c|}{ Peak \# } & Start Tube & End Tube \\
\hline $\begin{array}{l}1 \\
2 \\
3 \\
4 \\
5\end{array}$ & & $\begin{array}{c}A: 1 \\
A: 8 \\
A: 12 \\
A: 26 \\
A: 45\end{array}$ & $\begin{array}{c}A: 7 \\
A: 11 \\
A: 25 \\
A: 44 \\
A: 50\end{array}$ \\
\hline Duration & $\%$ B & Solvent A & Solvent B \\
\hline $\begin{array}{c}0.0 \\
1.0 \\
18.8\end{array}$ & $\begin{array}{c}0.0 \\
0.0 \\
23.5\end{array}$ & $\begin{array}{l}\text { A2 hexane } \\
\text { A2 hexane } \\
\text { A2 hexane }\end{array}$ & $\begin{array}{l}\text { B2 ethyl acetate } \\
\text { B2 ethyl acetate } \\
\text { B2 ethyl acetate }\end{array}$ \\
\hline
\end{tabular}

Page 1 of 1 


\section{Synthetic Methods}

\subsubsection{Hexyl 2-(hexyloxy)-5-((pyridin-2-ylmethyl)amino)benzoate: G5-051s10}

In a $20 \mathrm{~mL}$ vial, hexyl 5-amino-2-(hexyloxy)benzoate (G5-047s9, 82.95

mass\% w/EtOAc, 3 mmol, $1.1627 \mathrm{~g}$ ), and picolinaldehyde (3 mmol, $0.3213 \mathrm{~g}$, $0.2854 \mathrm{~mL})$ were dissolved in methanol $(10 \mathrm{~mL})$. The solution was stirred for 15 minutes, then $\mathrm{NaBH}_{3} \mathrm{CN}(3.3 \mathrm{mmol}, 0.2215 \mathrm{~g})$ was slowly added, and the reaction stirred for another 18 hours. The reaction was worked-up by adding $1 \mathrm{M} \mathrm{NaOH}(3$ $\mathrm{mL})$, then extracting with hexanes $(2 \times 3 \mathrm{~mL})$. The combined hexane phases were washed with water ( $3 \mathrm{~mL})$, then dried with $\mathrm{MgSO}_{4}$. After filtering, silica ( 2 g) was added to the filtrate. The mixture was concentrated by rotatory evaporation to a powder, which was used as a dry-load for silica chromatography (FIGURE 7.17).

Fractions 31-54 were combined and concentrated by rotatory evaporation to an oil (0.68 g, 55.0\% yield).

${ }^{1} \mathrm{H}-\mathrm{NMR} \delta(\mathrm{ppm})\left(\right.$ DMSO-d $\left._{6}\right):$ 8.50-8.54 $(1 \mathrm{H}, \mathrm{m}), 7.73(1 \mathrm{H}, \mathrm{td}, \mathrm{J}=7.67,1.82$ Hz), $7.35(1 \mathrm{H}, \mathrm{d}, \mathrm{J}=7.86 \mathrm{~Hz}), 7.22-7.27(1 \mathrm{H}, \mathrm{m}), 6.87(1 \mathrm{H}, \mathrm{d}, \mathrm{J}=8.92 \mathrm{~Hz}), 6.85$ $(1 \mathrm{H}, \mathrm{d}, \mathrm{J}=3.00 \mathrm{~Hz}), 6.71(1 \mathrm{H}, \mathrm{dd}, \mathrm{J}=8.87,3.02 \mathrm{~Hz}), 6.21(1 \mathrm{H}, \mathrm{t}, \mathrm{J}=6.16 \mathrm{~Hz})$, $4.32(2 \mathrm{H}, \mathrm{d}, \mathrm{J}=6.11 \mathrm{~Hz}), 4.15(2 \mathrm{H}, \mathrm{d}, \mathrm{J}=6.48 \mathrm{~Hz}), 3.84(2 \mathrm{H}, \mathrm{t}, \mathrm{J}=6.38 \mathrm{~Hz})$, 1.59-1.68 (4 H, m), 1.33-1.43 (4 H, m), 1.24-1.33 (8 H, m), 0.84-0.90 (6 H, m).

${ }^{13} \mathrm{C}-\mathrm{NMR} \delta$ (ppm)(DMSO-d 6 ): 166.6, 159.7, 148.9, 142.3, 136.6, 122.0, $121.7,121.0,116.7,115.9,113.8,69.3,64.2,49.0,31.0,30.9,28.9,28.2,25.2$ $25.1,22.1,22.0,13.9(2 \mathrm{C})$. 


\section{Synthetic Methods}

FIGURE 7.17: Chromatographic purification of G5-051s7.

Sample: grandy5-051s7

RediSep Column: Silica 40g SN: E04103BADFD88 Lot: 1922189010X

Flow Rate: $40 \mathrm{ml} / \mathrm{min}$

Equilibration Volume: 5.0 CV

Initial Waste: $0.0 \mathrm{CV}$

Air Purge: $1.0 \mathrm{~min}$

Solvent: A2 hexane

Solvent: B2 ethyl acetate
Rf 200 : OHSU COHEN RF200\#1

Friday 17 August 2012 02:18PM

Peak Tube Volume: Max.

Non-Peak Tube Volume: Max.

Loading Type: Solid

Wavelength 1 (red): 254nm

Peak Width: 2 min

Threshold: $0.05 \mathrm{AU}$

Wavelength 2 (purple): 280nm

Run Notes:

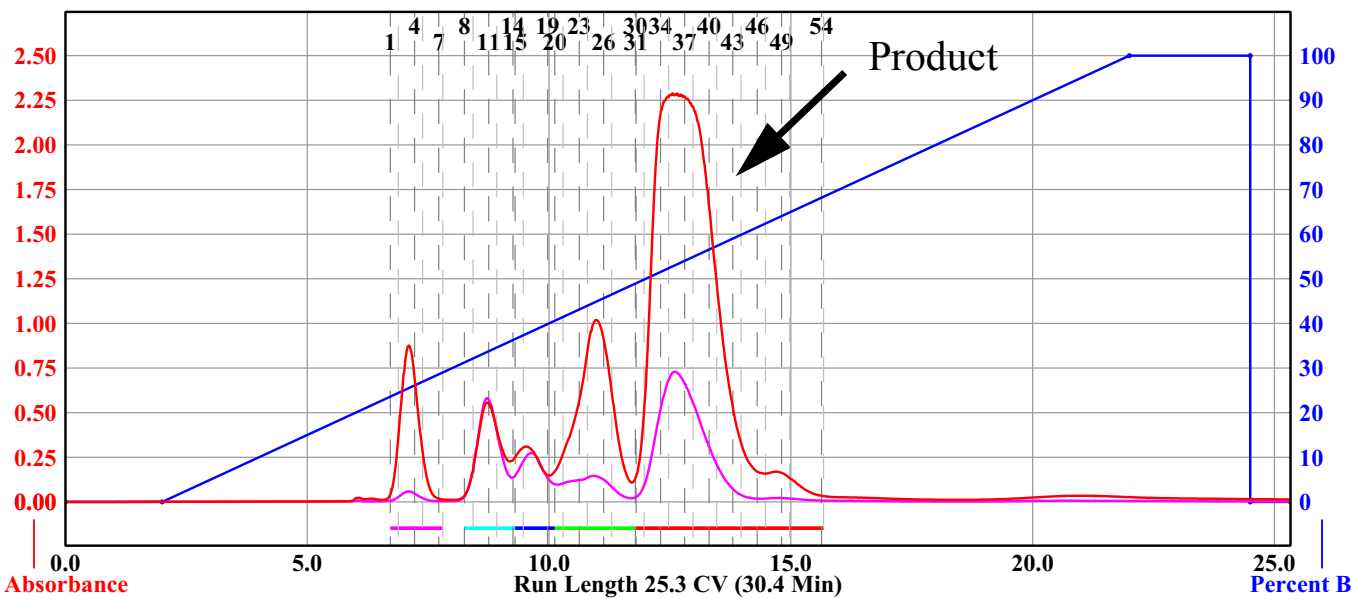

Rack A

\begin{tabular}{|c|c|c|c|c|c|}
\hline 108 & (107 & 106 & 105 & (14) & (103 \\
\hline (2) & 8 & (29) & 100 & (10) & (102 \\
\hline ש6 & (25) & (9) & (2) & (22) & (1) \\
\hline 85 & 80 & 87 & 88 & 89 & $\mathscr{D}$ \\
\hline 89 & 83 & (2) & (11) & (10) & (19) \\
\hline 13 & (2) & (2) & (20) & (2) & 18 \\
\hline (12) & (11) & (11) & (9) & 68 & (6) \\
\hline (1) & (2) & 63 & (9) & 65 & 60 \\
\hline (10 & 69 & 68 & (57) & 50 & 69 \\
\hline (4) & (1) & (1) & 6 & 6 & (3) \\
\hline (48) & (6) & (4) & (6) & (4) & (3) \\
\hline (3) & (B) & (2) & (1) & (1) & (4) \\
\hline (16) & (3) & (3) & (3) & (2) & (1) \\
\hline (2) & (26) & 87 & 28 & (29) & (BD \\
\hline (2) & (23) & (2) & (21) & (21) & - \\
\hline (1) & (1) & ○ & 0 & 0 & 0 \\
\hline (12) & (1) & (1) & (9) & (8) & (1) \\
\hline (1) & (2) & (3) & (4) & (5) & (6) \\
\hline
\end{tabular}

$13 \mathrm{~mm} \times 100 \mathrm{~mm}$ Tubes

\begin{tabular}{|c|c|c|c|}
\hline \multicolumn{2}{|c|}{ Peak \# } & Start Tube & End Tube \\
\hline $\begin{array}{l}1 \\
2 \\
3 \\
4 \\
5\end{array}$ & & $\begin{array}{c}A: 1 \\
A: 8 \\
A: 15 \\
A: 20 \\
A: 31\end{array}$ & $\begin{array}{c}\text { A:7 } \\
A: 14 \\
A: 19 \\
A: 30 \\
A: 54\end{array}$ \\
\hline Duration & $\%$ B & Solvent A & Solvent B \\
\hline $\begin{array}{c}0.0 \\
2.0 \\
20.0 \\
2.5 \\
0.0 \\
0.8\end{array}$ & $\begin{array}{c}0.0 \\
0.0 \\
100.0 \\
100.0 \\
0.0 \\
0.0\end{array}$ & $\begin{array}{l}\text { A2 hexane } \\
\text { A2 hexane } \\
\text { A2 hexane } \\
\text { A2 hexane } \\
\text { A2 hexane } \\
\text { A2 hexane }\end{array}$ & $\begin{array}{l}\text { B2 ethyl acetate } \\
\text { B2 ethyl acetate } \\
\text { B2 ethyl acetate } \\
\text { B2 ethyl acetate } \\
\text { B2 ethyl acetate } \\
\text { B2 ethyl acetate }\end{array}$ \\
\hline
\end{tabular}

Page 1 of 1 


\section{Synthetic Methods}

\subsubsection{Hexyl 5-((2-fluorobenzyl)amino)-2-(hexyloxy)benzoate: G5-052s10}

In a $20 \mathrm{~mL}$ vial, hexyl 5-amino-2-(hexyloxy)benzoate (G5-047s9, 82.95

mass\% w/EtOAc, $3 \mathrm{mmol}, 1.1617 \mathrm{~g}$ ), and 2-fluorobenzaldehyde (3 mmol, 0.3723

g, $0.316 \mathrm{~mL})$ were dissolved in methanol $(10 \mathrm{~mL})$. The solution was stirred for 15

minutes, then $\mathrm{NaBH}_{3} \mathrm{CN}(3.3 \mathrm{mmol}, 0.2070 \mathrm{~g})$ was slowly added, and the reaction

stirred for another 18 hours. The reaction was worked-up by adding $1 \mathrm{M} \mathrm{NaOH}$ (3

$\mathrm{mL})$, then extracting with hexanes $(2 \times 3 \mathrm{~mL})$. The combined hexane phases were washed with water $(3 \mathrm{~mL})$, then dried with $\mathrm{MgSO}_{4}$. After filtering, basic alumina $(\sim 2 \mathrm{~g})$ was added to the filtrate. The mixture was concentrated by rotatory evaporation to a powder, which was used as a dry-load for silica chromatography (FIG-

URE 7.18). Fractions 13-31 were combined and concentrated by rotatory evaporation to an oil $(0.7858 \mathrm{~g}, 61.0 \%$ yield $)$.

${ }^{1} \mathrm{H}-\mathrm{NMR} \delta(\mathrm{ppm})\left(\mathrm{DMSO}_{6}\right): 7.39(1 \mathrm{H}, \mathrm{t}, \mathrm{J}=7.72 \mathrm{~Hz}), 7.25-7.33(1 \mathrm{H}, \mathrm{m})$, 7.11-7.21 (2 H, m), $6.88(1 \mathrm{H}, \mathrm{d}, \mathrm{J}=8.92 \mathrm{~Hz}), 6.87(1 \mathrm{H}, \mathrm{d}, \mathrm{J}=3.00 \mathrm{~Hz}), 6.73(1 \mathrm{H}$, dd, $J=8.88,3.04 \mathrm{~Hz}), 6.07(1 \mathrm{H}, \mathrm{t}, \mathrm{J}=6.18 \mathrm{~Hz}), 4.27(2 \mathrm{H}, \mathrm{d}, \mathrm{J}=6.12 \mathrm{~Hz}), 4.15(2$ $\mathrm{H}, \mathrm{t}, \mathrm{J}=6.52 \mathrm{~Hz}), 3.85(2 \mathrm{H}, \mathrm{t}, \mathrm{J}=6.34 \mathrm{~Hz}), 1.58-1.69(4 \mathrm{H}, \mathrm{m}), 1.33-1.44(4 \mathrm{H}$, m), 1.22-1.33 (8 H, m), 0.81-0.93 (6 H, m).

${ }^{13} \mathrm{C}-\mathrm{NMR} \delta(\mathrm{ppm})\left(\mathrm{DMSO}_{6}\right): 166.6,160.3(1 \mathrm{C}, \mathrm{d}, \mathrm{J}=243.65 \mathrm{~Hz}), 148.9$, 142.2, $129.3(1 \mathrm{C}, \mathrm{d}, \mathrm{J}=4.55 \mathrm{~Hz}), 128.6(1 \mathrm{C}, \mathrm{d}, \mathrm{J}=8.16 \mathrm{~Hz}), 126.6(1 \mathrm{C}, \mathrm{d}, \mathrm{J}=$ $14.64 \mathrm{~Hz}), 124.3(1 \mathrm{C}, \mathrm{d}, \mathrm{J}=3.20 \mathrm{~Hz}), 121.6,116.6,115.9,115.0(1 \mathrm{C}, \mathrm{d}, \mathrm{J}=21.31$ 


\section{Synthetic Methods}

$\mathrm{Hz}), 113.7,69.3,64.2,40.4(1 \mathrm{C}, \mathrm{d}, \mathrm{J}=3.88 \mathrm{~Hz}), 31.0,30.9,28.9,28.2,25.2(2 \mathrm{C})$,

$22.1,22.0,13.9(2 \mathrm{C})$. 


\section{Synthetic Methods}

FIGURE 7.18: Chromatographic purification of G5-052s7.

Sample: grandy5-052s7

RediSep Column: Silica 24g SN: E041039E8CEB1 Lot: 1920177010W Flow Rate: $35 \mathrm{ml} / \mathrm{min}$

Equilibration Volume: 5.0 CV

Initial Waste: $0.0 \mathrm{CV}$

Air Purge: $1.0 \mathrm{~min}$

Solvent: A2 hexane

Solvent: B2 ethyl acetate
Rf 200 : OHSU COHEN RF200\#1

Thursday 16 August 2012 03:11PM

Peak Tube Volume: Max.

Non-Peak Tube Volume: Max.

Loading Type: Solid (Pause)

Wavelength 1 (red): $254 \mathrm{~nm}$

Peak Width: 1 min

Threshold: 0.05 AU

Wavelength 2 (purple): 280nm

Run Notes:

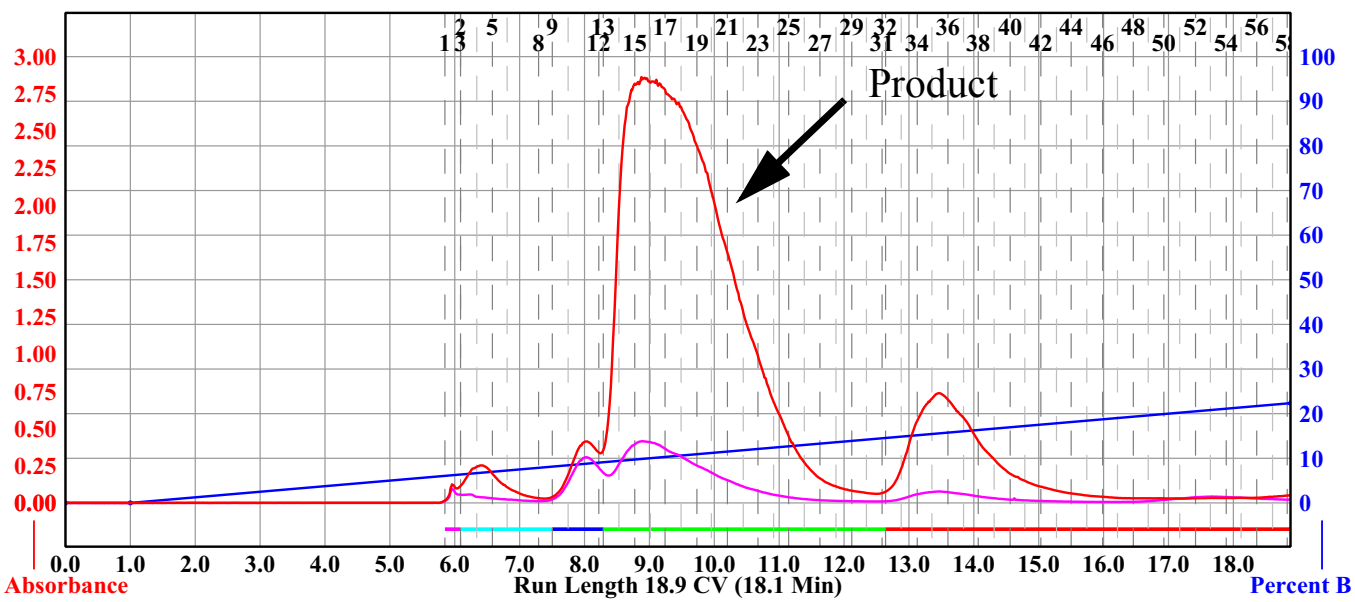

Rack A

\begin{tabular}{|c|c|c|c|c|c|}
\hline 108 & (107) & 106 & 105 & (104 & $1(13)$ \\
\hline 90 & $\mathscr{B}$ & 99 & (100) & (1) & (102 \\
\hline ஜூ & (2) & அ & Q3 & (2) & (21) \\
\hline 85 & 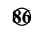 & 87 & 88 & 89 & (1) \\
\hline 8 & 83 & $(12$ & (11) & (81) & (19) \\
\hline 13 & (2) & (1) & (2) & (12) & 18 \\
\hline (12) & (11) & (11) & (9) & 8 & (67) \\
\hline (11) & (2) & 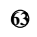 & (6) & (1) & ø \\
\hline (10 & 59 & 68 & 67 & 6 & (5) \\
\hline (1) & (1) & (1) & (2) & 6 & 6 \\
\hline (4) & 0 & (6) & (6) & $\theta$ & (3) \\
\hline (b) & (3) & (2) & (1) & (1) & (1) \\
\hline (1) & (5) & (3) & (3) & (1) & (11) \\
\hline (2) & (26) & 07 & 8 & 29 & (10) \\
\hline 89 & (23) & (2) & (1) & (21) & (1) \\
\hline (1) & (1) & (1) & (16) & 0 & 0 \\
\hline 0 & 0 & 0 & 0 & (8) & (1) \\
\hline (1) & (2) & (3) & (4) & (5) & (6) \\
\hline
\end{tabular}

$13 \mathrm{~mm} \times 100 \mathrm{~mm}$ Tubes

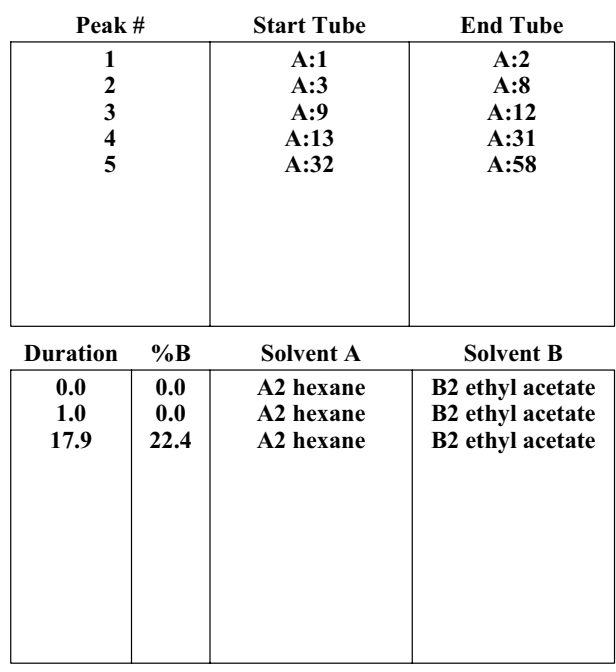

Page 1 of 1 


\section{Synthetic Methods}

\subsubsection{Hexyl 5-((3-fluorobenzyl)amino)-2-(hexyloxy)benzoate: G5-053s10}

In a $20 \mathrm{~mL}$ vial, hexyl 5-amino-2-(hexyloxy)benzoate (G5-047s9, 82.95

mass\% w/EtOAc, $3 \mathrm{mmol}, 1.1658 \mathrm{~g}$ ), and 3-fluorobenzaldehyde (3 mmol, 0.3723

$\mathrm{g}, 0.3182 \mathrm{~mL})$ were dissolved in methanol $(10 \mathrm{~mL})$. The solution was stirred for 15 minutes, then $\mathrm{NaBH}_{3} \mathrm{CN}(3.3 \mathrm{mmol}, 0.20 \mathrm{~g}$ ) was slowly added, and the reaction stirred for another 18 hours. The reaction was worked-up by adding $1 \mathrm{M} \mathrm{NaOH}$ (3 $\mathrm{mL})$, then extracting with hexanes $(2 \times 3 \mathrm{~mL})$. The combined hexane phases were washed with water $(3 \mathrm{~mL})$, then dried with $\mathrm{MgSO}_{4}$. After filtering, basic alumina $(\sim 2 \mathrm{~g})$ was added to the filtrate. The mixture was concentrated by rotatory evaporation to a powder, which was used as a dry-load for silica chromatography (FIG-

URE 7.19). Fractions 11-26 were combined and concentrated by rotatory evaporation to an oil (0.7699 $\mathrm{g}, 59.7 \%$ yield $)$.

${ }^{1} \mathrm{H}-\mathrm{NMR} \delta(\mathrm{ppm})\left(\mathrm{DMSO}_{6}\right): 7.32-7.40(1 \mathrm{H}, \mathrm{m}), 7.19(1 \mathrm{H}, \mathrm{d}, \mathrm{J}=7.68 \mathrm{~Hz})$, 7.11-7.17 (1 H, m), 7.04 (1 H, td, J = 8.61, 2.57 Hz), $6.87(1 \mathrm{H}, \mathrm{d}, \mathrm{J}=8.86 \mathrm{~Hz})$, $6.84(1 \mathrm{H}, \mathrm{d}, \mathrm{J}=2.96 \mathrm{~Hz}), 6.70(1 \mathrm{H}, \mathrm{dd}, \mathrm{J}=8.87,2.98 \mathrm{~Hz}), 6.17(1 \mathrm{H}, \mathrm{t}, \mathrm{J}=6.21$ Hz), $4.26(2 \mathrm{H}, \mathrm{d}, \mathrm{J}=6.16 \mathrm{~Hz}), 4.15(2 \mathrm{H}, \mathrm{t}, \mathrm{J}=6.49 \mathrm{~Hz}), 3.84(2 \mathrm{H}, \mathrm{t}, \mathrm{J}=6.37 \mathrm{~Hz})$, 1.58-1.68 (4 H, m), 1.33-1.43 (4 H, m), 1.24-1.33 (8 H, m), 0.83-0.90 (6 H, m).

${ }^{13} \mathrm{C}-\mathrm{NMR} \delta(\mathrm{ppm})\left(\mathrm{DMSO}_{6}\right): 166.6,162.3(1 \mathrm{C}, \mathrm{d}, \mathrm{J}=243.38 \mathrm{~Hz}), 148.9$, 143.5 (1 C, d, J = 6.75 Hz), 142.2, $130.1(1 \mathrm{C}, \mathrm{d}, \mathrm{J}=8.24 \mathrm{~Hz}), 123.0$ (1 C, d, J = 2.34 Hz), 121.6, 116.3 (1 C, d, J = 88.03 Hz), 113.9, 113.7, 113.5 (1 C, d, J = 8.78 


\section{Synthetic Methods}

Hz), 113.2, 69.3, 64.2, 46.4, 31.0, 30.9, 28.9, 28.2, 25.2, 25.1, 22.1, 22.0, 13.9 (2

C). 


\section{Synthetic Methods}

FIGURE 7.19: Chromatographic purification of G5-053s7.

Sample: grandy5-053s7

RediSep Column: Silica 24g SN: E041037FE8E71 Lot: 1916333010W Flow Rate: $35 \mathrm{ml} / \mathrm{min}$

Equilibration Volume: 5.0 CV

Initial Waste: $0.0 \mathrm{CV}$

Air Purge: $1.0 \mathrm{~min}$

Solvent: A2 hexane

Solvent: B2 ethyl acetate
Rf 200 : OHSU COHEN RF200\#1

Thursday 16 August 2012 02:33PM

Peak Tube Volume: Max.

Non-Peak Tube Volume: Max.

Loading Type: Solid

Wavelength 1 (red): 254nm

Peak Width: 1 min

Threshold: 0.05 AU

Wavelength 2 (purple): 280nm

Run Notes:

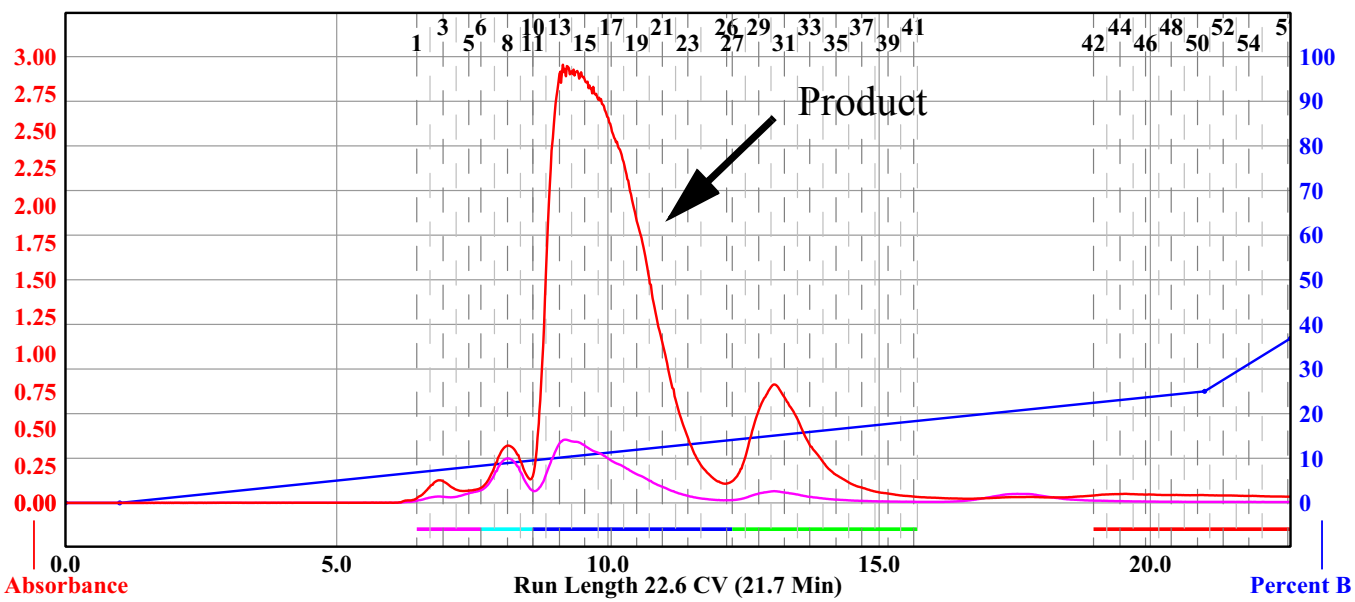

Rack A

\begin{tabular}{|c|c|c|c|c|c|}
\hline 108 & (107 & 106 & 105 & (144) & (103 \\
\hline (2) & $\mathscr{0}$ & $\mathscr{9 9}$ & (100 & (10) & (102 \\
\hline (ன & 98 & (2) & (23) & (2) & (21) \\
\hline 87 & 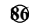 & 87 & 88 & 89 & (20 \\
\hline 8 & 83 & (32) & (11) & (10) & (1) \\
\hline$(3)$ & (8) & 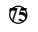 & (2) & (27) & 88 \\
\hline (12) & (11) & (11) & 69 & 68 & (6) \\
\hline (1) & (2) & 63 & 6 & 67 & ஐ \\
\hline (1) & 59 & 68 & 67 & 6 & 6 \\
\hline (9) & (1) & 0 & 0 & 6 & 6 \\
\hline (4) & 0 & (4) & (6) & 4 & (4) \\
\hline (3) & $B$ & (3) & (41) & (41) & (4) \\
\hline (6) & (3) & (3) & (3) & (32) & (11) \\
\hline 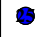 & - & 87 & 28 & 29 & (10) \\
\hline & 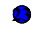 & 0 & 0 & & 0 \\
\hline 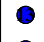 & 0 & 0 & 0 & 0 & - \\
\hline 0 & 0 & (11) & (9) & (8) & (7) \\
\hline (1) & (2) & (3) & (4) & (5) & (6) \\
\hline
\end{tabular}

$13 \mathrm{~mm} \times 100 \mathrm{~mm}$ Tubes

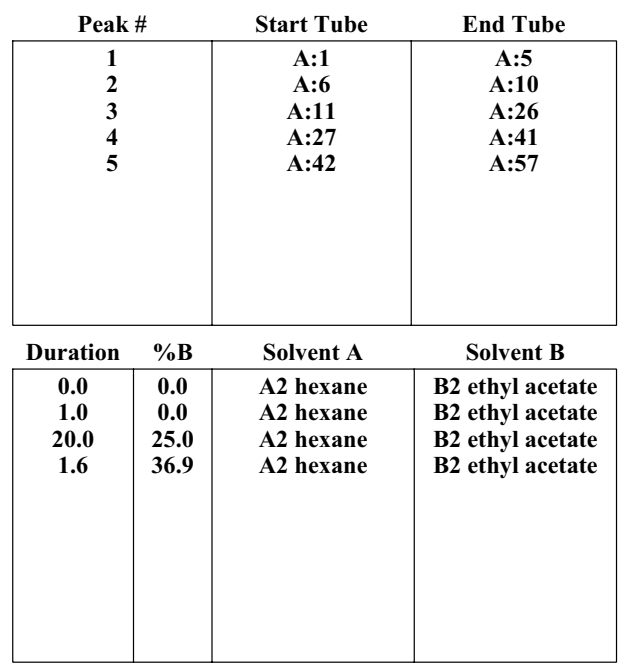

Page 1 of 1 


\section{Synthetic Methods}

7.1.2.24 (5-(benzylamino)-2-(hexyloxy)phenyl)(pyrrolidin-1-yl)methanone: G5103s10

In a $20 \mathrm{~mL}$ vial, (5-amino-2-(hexyloxy)phenyl)(pyrrolidin-1-yl)methanone

(G5-101s5, $1 \mathrm{mmol}, 0.290 \mathrm{~g})$, and benzaldehyde (1 mmol, $0.1061 \mathrm{~g}, 0.102 \mathrm{~mL}$ )

were dissolved in methanol $(10 \mathrm{~mL})$. The solution was stirred for 15 minutes, then

$\mathrm{NaBH}_{3} \mathrm{CN}$ (1.1 mmol, $0.0691 \mathrm{~g}$ ) was added, and the reaction stirred for another 18

hours. The reaction was worked-up by adding $1 \mathrm{M} \mathrm{NaOH}(3 \mathrm{~mL})$, then extracted

with hexanes $(5 \times 3 \mathrm{~mL})$. The combined hexane phases were washed with water $(3$

$\mathrm{mL}$ ), then dried with $\mathrm{MgSO}_{4}$. The hexane extractions were not effective, so the

aqueous phase was diluted with water $(15 \mathrm{~mL})$, then extracted with ethyl acetate

(EtOAc, $3 \times 10 \mathrm{~mL}$ ), and finally dried with $\mathrm{MgSO}_{4}$. After filtering, the haxene and

EtOAc solutions were combined, and concentrated by rotatory evaporation to a

yellow oil, which was purified by silica chromatography (FIGURE 7.20). Fractions

15-30 were combined and concentrated by rotatory evaporation.

${ }^{1} \mathrm{H}-\mathrm{NMR} \delta(\mathrm{ppm})\left(\right.$ DMSO-d $\left._{6}\right): 7.29-7.38(4 \mathrm{H}, \mathrm{m}), 7.19-7.25(1 \mathrm{H}, \mathrm{m}), 6.79$ (1

$\mathrm{H}, \mathrm{d}, \mathrm{J}=8.83 \mathrm{~Hz}), 6.54(1 \mathrm{H}, \mathrm{dd}, \mathrm{J}=8.83,2.88 \mathrm{~Hz}), 6.40(1 \mathrm{H}, \mathrm{d}, \mathrm{J}=2.86 \mathrm{~Hz})$,

$5.95(1 \mathrm{H}, \mathrm{t}, \mathrm{J}=6.06 \mathrm{~Hz}), 4.21(2 \mathrm{H}, \mathrm{d}, \mathrm{J}=6.01 \mathrm{~Hz}), 3.82(2 \mathrm{H}, \mathrm{t}, \mathrm{J}=6.22 \mathrm{~Hz}), 3.38$

$(2 \mathrm{H}, \mathrm{t}, \mathrm{J}=6.82 \mathrm{~Hz}), 3.06(2 \mathrm{H}, \mathrm{m}), 1.70-1.87$ (4 H, m), 1.54-1.63 (2 H, m), 1.32-

$1.39(2 \mathrm{H}, \mathrm{m}), 1.23-1.32(4 \mathrm{H}, \mathrm{m}), 0.84-0.90(3 \mathrm{H}, \mathrm{t}, \mathrm{J}=6.66 \mathrm{~Hz})$. 


\section{Synthetic Methods}

FIGURE 7.20: Chromatographic purification of G5-103s8.

Sample: grandy5-103s8

RediSep Column: Silica 40g SN: E0410597A508F Lot: 2112259020X

Flow Rate: $40 \mathrm{ml} / \mathrm{min}$

Equilibration Volume: 5.0 CV

Initial Waste: $0.0 \mathrm{CV}$

Air Purge: $1.0 \mathrm{~min}$

Solvent: A1 hexane

Solvent: B1 ethyl acetate
Rf 200 : PDX-PEYTON RF200\#1

Monday 01 October 2012 11:13AM

Peak Tube Volume: Max.

Non-Peak Tube Volume: Max.

Loading Type: Solid (Pause)

Wavelength 1 (red): 254nm

Peak Width: 2 min

Threshold: 0.05 AU

Wavelength 2 (purple): 280nm

Run Notes:

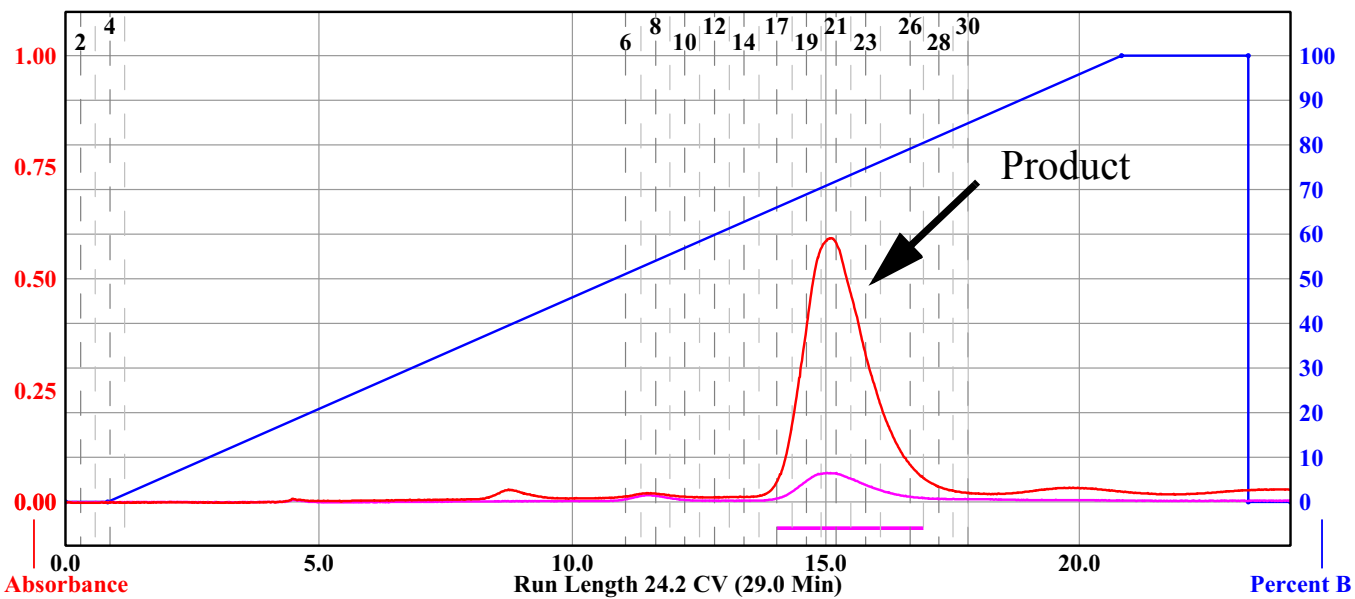

\begin{tabular}{|c|c|c|c|c|}
\hline \multicolumn{5}{|c|}{ Rack A } \\
\hline (71) & (72) & (73) & (74) & (75) \\
\hline (70) & (9) & 68 & (67) & 60 \\
\hline (61) & (62) & (63) & (64) & (65) \\
\hline (6) & (59) & 58 & (57) & (56) \\
\hline (51) & (52) & (53) & (54) & (55) \\
\hline (50) & (49) & (48) & (47) & (46) \\
\hline (41) & (42) & (43) & (44) & (45) \\
\hline (40) & (39) & (38) & (37) & (36) \\
\hline (31) & (32) & (33) & (34) & (35) \\
\hline (30) & (29) & (28) & (27) & (26) \\
\hline (21) & (22) & (23) & (24) & (25) \\
\hline (20) & (19) & (18) & (17) & (1) \\
\hline (11) & (12) & (13) & (14) & (15) \\
\hline (10) & (9) & (8) & (7) & 6 \\
\hline (1) & (2) & (3) & (4) & 5 \\
\hline
\end{tabular}

\begin{tabular}{|c|c|c|c|}
\hline \multicolumn{2}{|c|}{ Peak \# } & Start Tube & End Tube \\
\hline \multicolumn{2}{|l|}{1} & \multirow[t]{2}{*}{ A:17 } & \multirow[t]{2}{*}{ A:26 } \\
\hline Duration & $\%$ B & & \\
\hline 0.0 & 0.0 & A1 hexane & B1 ethyl acetate \\
\hline 0.8 & 0.0 & A1 hexane & B1 ethyl acetate \\
\hline 20.0 & 100.0 & A1 hexane & B1 ethyl acetate \\
\hline 2.5 & 100.0 & A1 hexane & B1 ethyl acetate \\
\hline 0.0 & 0.0 & A1 hexane & B1 ethyl acetate \\
\hline 0.8 & 0.0 & A1 hexane & B1 ethyl acetate \\
\hline & & & \\
\hline & & & \\
\hline & & & \\
\hline
\end{tabular}

Page 1 of 1 


\section{Synthetic Methods}

\subsubsection{5 (5-((4-fluorobenzyl)amino)-2-(hexyloxy)phenyl)(pyrrolidin-1-}

yl)methanone: G5-108s10

In a $20 \mathrm{~mL}$ vial, (5-amino-2-(hexyloxy)phenyl)(pyrrolidin-1-yl)methanone

(G5-106s7, $1 \mathrm{mmol}, 0.2889 \mathrm{~g})$, and 4-fluorobenzaldehyde (1 mmol, $0.1241 \mathrm{~g}$, $0.1073 \mathrm{~mL})$ were dissolved in methanol $(10 \mathrm{~mL})$. The solution was stirred for 15 minutes, then $\mathrm{NaBH}_{3} \mathrm{CN}$ (1.59 mmol, $0.10 \mathrm{~g}$ ) was added, and the reaction stirred for another 18 hours. The reaction was worked-up by adding $1 \mathrm{M} \mathrm{NaOH}(3 \mathrm{~mL})$, then extracted with ethyl acetate (EtOAc, 2 x $5 \mathrm{~mL}$ ), and finally dried with $\mathrm{MgSO}_{4}$. After filtering, silica gel ( $\left.\sim \mathrm{g}\right)$ was added to the filtrate. The mixture was concentrated by rotatory evaporation to a powder, which was used as a dry-load for by silica chromatography (FIGURE 7.21). Fractions 1-27 were combined, using dichloromethane to aid the transfer, and concentrated by rotatory evaporation (0.4902 g gross, 42.9 mol\% w/EtOAc by NMR, 77.23 mass\%, 0.3786 g net, $\sim 95 \%$ yield).

${ }^{1} \mathrm{H}-\mathrm{NMR} \delta(\mathrm{ppm})\left(\mathrm{DMSO}_{6}\right):$ 7.35-7.41 (2 H, m), 7.10-7.17 (2 H, m), 6.79 (1 $\mathrm{H}, \mathrm{d}, \mathrm{J}=8.83 \mathrm{~Hz}), 6.53(1 \mathrm{H}, \mathrm{d}, \mathrm{J}=8.86,2.90 \mathrm{~Hz}), 6.39(1 \mathrm{H}, \mathrm{d}, \mathrm{J}=2.86 \mathrm{~Hz}), 5.96$ $(1 \mathrm{H}, \mathrm{t}, \mathrm{J}=6.12 \mathrm{~Hz}), 4.20(2 \mathrm{H}, \mathrm{d}, \mathrm{J}=6.14 \mathrm{~Hz}), 3.82(2 \mathrm{H}, \mathrm{t}, \mathrm{J}=6.26 \mathrm{~Hz}), 3.37(2$ $\mathrm{H}, \mathrm{t}, \mathrm{J}=6.78 \mathrm{~Hz}), 3.00-3.13(2 \mathrm{H}, \mathrm{m}), 1.70-1.83(4 \mathrm{H}, \mathrm{m}), 1.54-1.65(2 \mathrm{H}, \mathrm{m})$, 1.30-1.39 (2 H, m), 1.22-1.30 (4 H, m), 0.87 (3 H, t, J = 6.73 Hz). 


\section{Synthetic Methods}

FIGURE 7.21: Chromatographic purification of G5-108s7.

Sample: grandy5-108s 7

RediSep Column: Silica 40g

Flow Rate: $40 \mathrm{ml} / \mathrm{min}$

Equilibration Volume: $240.0 \mathrm{ml}$

Initial Waste: $0.0 \mathrm{ml}$

Air Purge: $1.0 \mathrm{~min}$

Solvent: A2 hexane

Solvent: B2 ethyl acetate
Rf 200 : OHSU COHEN RF200\#1

Peak Tube Volume: Max.

Non-Peak Tube Volume: Max.

Loading Type: Solid

Wavelength 1 (red): 254nm

Threshold: 0.05 AU

Wavelength 2 (purple): 280nm
Peak Width: 2 min

\section{Wednesday 10 October 2012 02:52PM}

Run Notes:

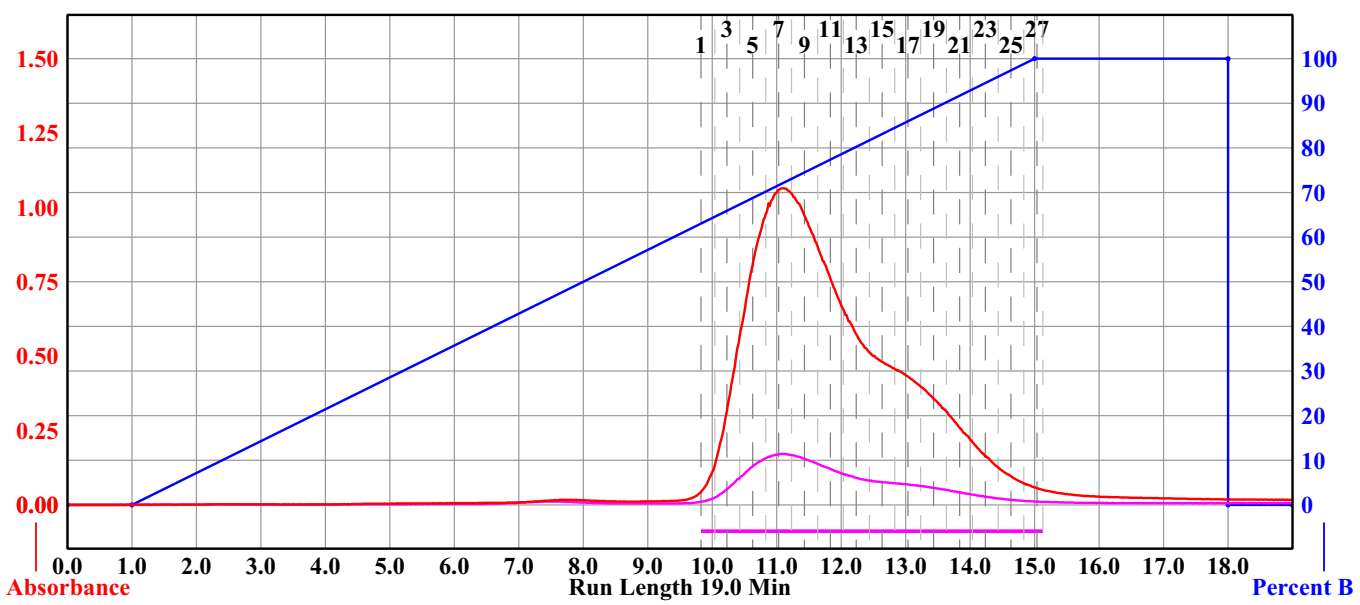

Rack A

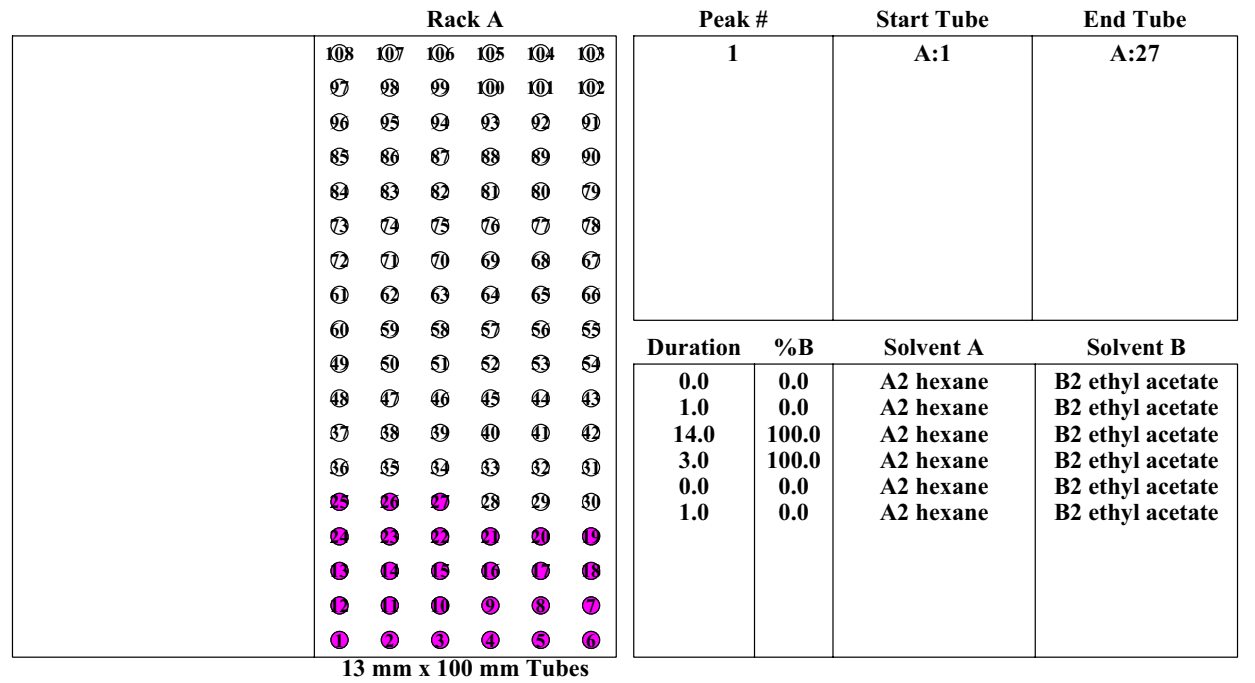

Page 1 of 1 


\section{Synthetic Methods}

\subsubsection{6 (5-((3-fluorobenzyl)amino)-2-(hexyloxy)phenyl)(pyrrolidin-1-}

yl)methanone: G5-109s8

In a $20 \mathrm{~mL}$ vial, (5-amino-2-(hexyloxy)phenyl)(pyrrolidin-1-yl)methanone

(G5-106s7, $1 \mathrm{mmol}, 0.2990 \mathrm{~g}$ ), and 3-fluorobenzaldehyde (1 mmol, $0.1241 \mathrm{~g}$, $0.1061 \mathrm{~mL})$ were dissolved in methanol $(10 \mathrm{~mL})$. The solution was stirred for 15 minutes, then $\mathrm{NaBH}_{3} \mathrm{CN}$ (1.59 mmol, $0.10 \mathrm{~g}$ ) was added, and the reaction stirred for another 18 hours. The reaction was worked-up by adding $1 \mathrm{M} \mathrm{NaOH}(3 \mathrm{~mL})$, then extracted with ethyl acetate (EtOAc, 2 x $5 \mathrm{~mL}$ ), and finally dried with $\mathrm{MgSO}_{4}$. After filtering, silica gel ( $\left.\sim 2 \mathrm{~g}\right)$ was added to the filtrate. The mixture was concentrated by rotatory evaporation to a powder, which was used as a dry-load for by silica chromatography (FIGURE 7.22). Fractions 1-18 were combined, using dichloromethane to aid the transfer, and concentrated by rotatory evaporation.

${ }^{1} \mathrm{H}-\mathrm{NMR} \delta(\mathrm{ppm})\left(\right.$ DMSO-d $\left._{6}\right):$ 7.31-7.40 (1 H, m), 7.17-7.21 (1 H, m), 7.127.17 (1 H, m), 7.00-7.07 (1 H, m), 6.80 (1 H, d, J = 8.84 Hz), 6.53 (1 H, dd, J = 8.83, $2.89 \mathrm{~Hz}), 6.39(1 \mathrm{H}, \mathrm{d}, \mathrm{J}=2.86 \mathrm{~Hz}), 6.02(1 \mathrm{H}, \mathrm{t}, \mathrm{J}=6.23 \mathrm{~Hz}), 4.24(2 \mathrm{H}, \mathrm{d}, \mathrm{J}$ $=6.18 \mathrm{~Hz}), 3.82(2 \mathrm{H}, \mathrm{t}, \mathrm{J}=6.22 \mathrm{~Hz}), 3.38(2 \mathrm{H}, \mathrm{t}, \mathrm{J}=6.80 \mathrm{~Hz}), 3.06(2 \mathrm{H}, \mathrm{m})$, 1.71-1.87 (4 H, m), 1.54-1.64 (2 H, m), 1.31-1.39 (2 H, m), 1.23-1.31 (4 H, m), $0.87(3 \mathrm{H}, \mathrm{t}, \mathrm{J}=6.72 \mathrm{~Hz})$. 


\section{Synthetic Methods}

FIGURE 7.22: Chromatographic purification of G5-109s7.

Sample: grandy5-109s7

RediSep Column: Silica 40g

Flow Rate: $40 \mathrm{ml} / \mathrm{min}$

Equilibration Volume: $240.0 \mathrm{ml}$

Initial Waste: $0.0 \mathrm{ml}$

Air Purge: $1.0 \mathrm{~min}$

Solvent: A2 hexane

Solvent: B2 ethyl acetate
Rf 200 : OHSU COHEN RF200\#1

Peak Tube Volume: Max.

Non-Peak Tube Volume: Max.

Loading Type: Solid

Wavelength 1 (red): 254nm

Threshold: $0.05 \mathrm{AU}$

Wavelength 2 (purple): 280nm
Peak Width: 2 min

\section{Wednesday 10 October 2012 03:27PM}

Run Notes:

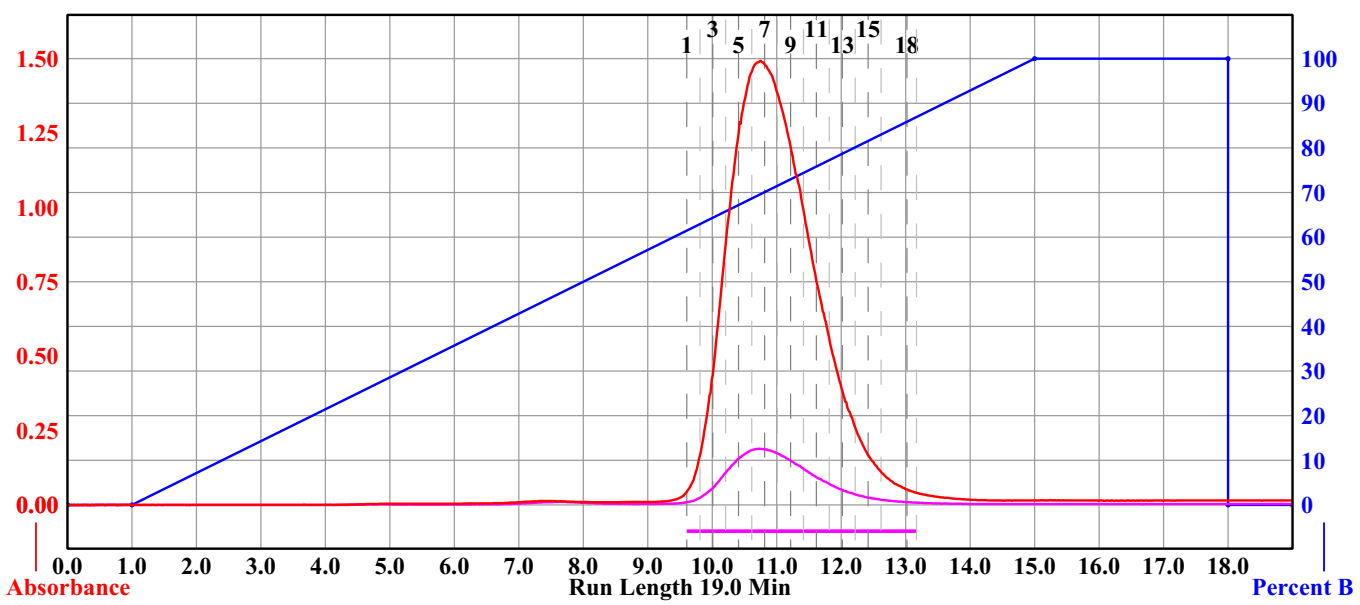

Rack A

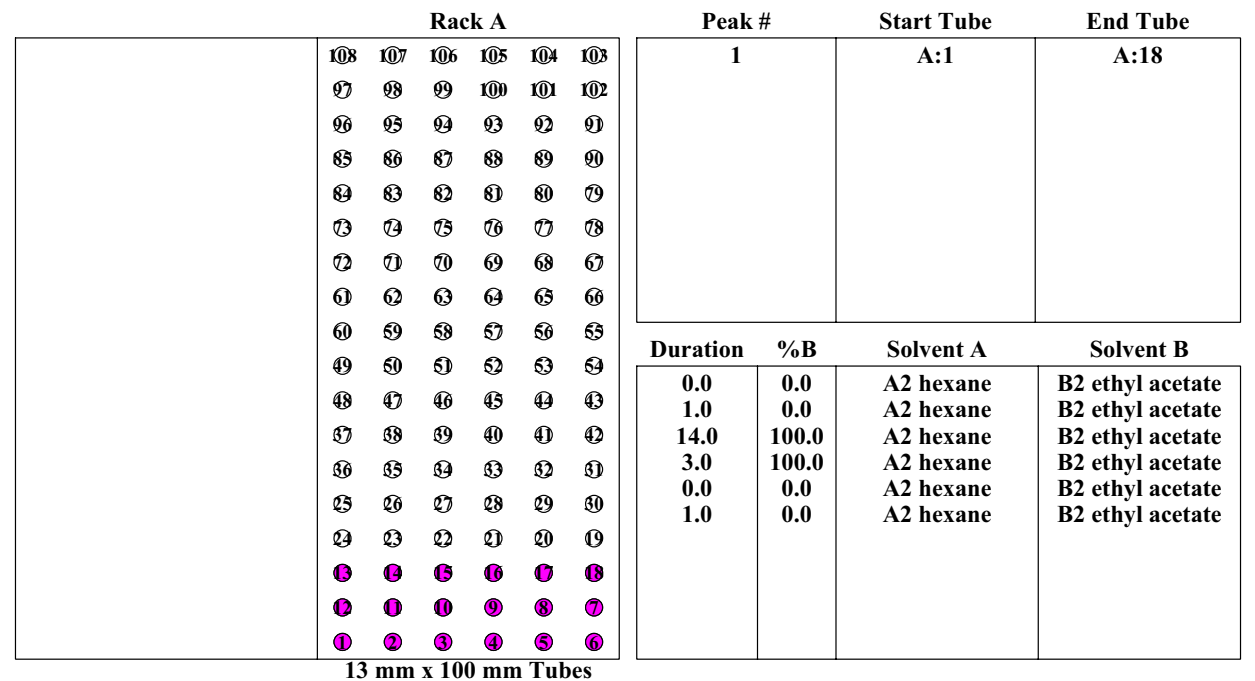

Page 1 of 1 


\section{Synthetic Methods}

\subsubsection{7 (5-((2-fluorobenzyl)amino)-2-(hexyloxy)phenyl)(pyrrolidin-1-}

yl)methanone: G5-110s8

In a $20 \mathrm{~mL}$ vial, (5-amino-2-(hexyloxy)phenyl)(pyrrolidin-1-yl)methanone

(G5-106s7, $1 \mathrm{mmol}, 0.2857 \mathrm{~g}$ ), and 2-fluorobenzaldehyde (1 mmol, $0.1241 \mathrm{~g}$, $0.1053 \mathrm{~mL})$ were dissolved in methanol $(10 \mathrm{~mL})$. The solution was stirred for 15 minutes, then $\mathrm{NaBH}_{3} \mathrm{CN}$ (1.59 mmol, $0.10 \mathrm{~g}$ ) was added, and the reaction stirred for another 18 hours. The reaction was worked-up by adding $1 \mathrm{M} \mathrm{NaOH}(3 \mathrm{~mL})$, then extracted with ethyl acetate (EtOAc, 2 x $5 \mathrm{~mL}$ ), and finally dried with $\mathrm{MgSO}_{4}$. After filtering, silica gel ( $\left.\sim 2 \mathrm{~g}\right)$ was added to the filtrate. The mixture was concentrated by rotatory evaporation to a powder, which was used as a dry-load for by silica chromatography (FIGURE 7.23). Fractions 1-19 were combined, using dichloromethane to aid the transfer, and concentrated by rotatory evaporation.

${ }^{1} \mathrm{H}-\mathrm{NMR} \delta(\mathrm{ppm})\left(\right.$ DMSO-d $\left._{6}\right):$ 7.37-7.43 (1 H, m), 7.25-7.33 (1 H, m), 7.12-

$7.21(2 \mathrm{H}, \mathrm{m}), 6.81(1 \mathrm{H}, \mathrm{d}, \mathrm{J}=8.82 \mathrm{~Hz}), 6.56(1 \mathrm{H}, \mathrm{dd}, \mathrm{J}=8.82,2.89 \mathrm{~Hz}), 6.42(1$ $\mathrm{H}, \mathrm{d}, \mathrm{J}=2.85 \mathrm{~Hz}), 5.91(1 \mathrm{H}, \mathrm{t}, \mathrm{J}=6.15 \mathrm{~Hz}), 4.25(2 \mathrm{H}, \mathrm{d}, \mathrm{J}=6.08 \mathrm{~Hz}), 3.83(2 \mathrm{H}$, $\mathrm{t}, \mathrm{J}=6.20 \mathrm{~Hz}), 3.38(2 \mathrm{H}, \mathrm{t}, \mathrm{J}=6.74 \mathrm{~Hz}), 3.03-3.12(2 \mathrm{H}, \mathrm{m}), 1.71-1.88(4 \mathrm{H}, \mathrm{m})$, 1.54-1.64 (2 H, m), 1.31-1.40 (2 H, m), 1.23-1.31 (4 H, m), $0.87(3 \mathrm{H}, \mathrm{t}, \mathrm{J}=6.53$ $\mathrm{Hz})$. 


\section{Synthetic Methods}

FIGURE 7.23: Chromatographic purification of G5-110s7.

Sample: grandy5-110s7

RediSep Column: Silica 40g

Flow Rate: $40 \mathrm{ml} / \mathrm{min}$

Equilibration Volume: $240.0 \mathrm{ml}$

Initial Waste: $0.0 \mathrm{ml}$

Air Purge: $1.0 \mathrm{~min}$

Solvent: A2 hexane

Solvent: B2 ethyl acetate
Rf 200 : OHSU COHEN RF200\#1

Peak Tube Volume: Max.

Non-Peak Tube Volume: Max.

Loading Type: Solid

Wavelength 1 (red): 254nm

Peak Width: 2 min

Threshold: $0.05 \mathrm{AU}$

Wavelength 2 (purple): 280nm
Wednesday 07 November 2012 02:28PM

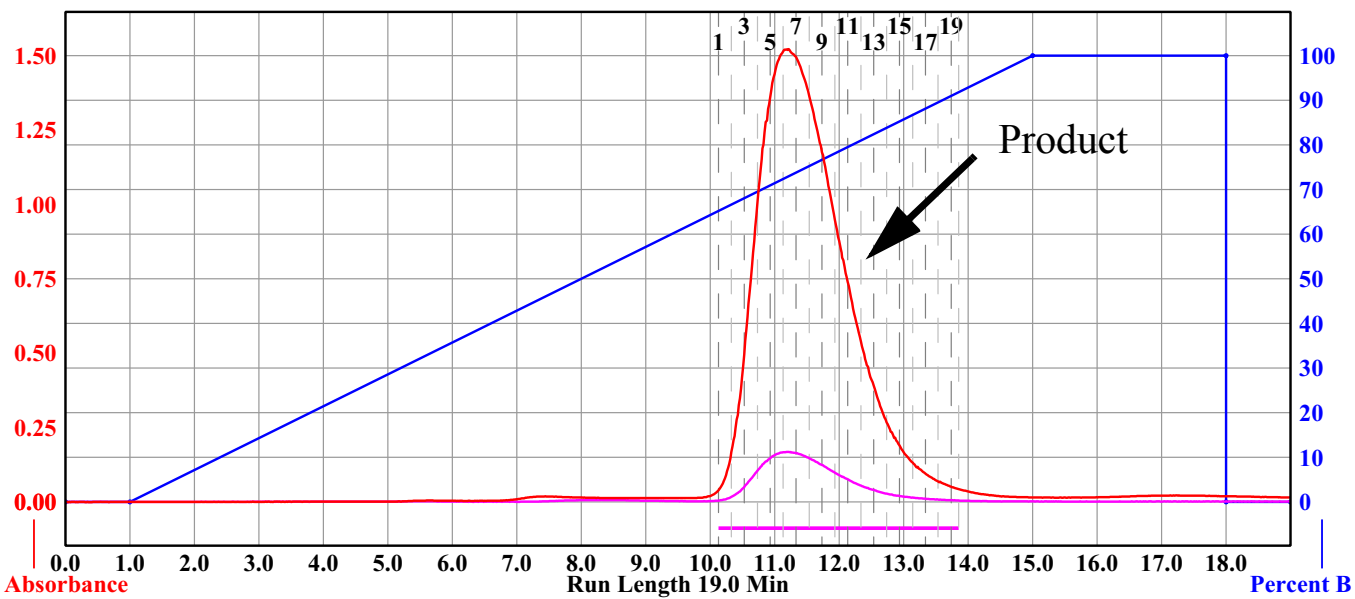

Rack A

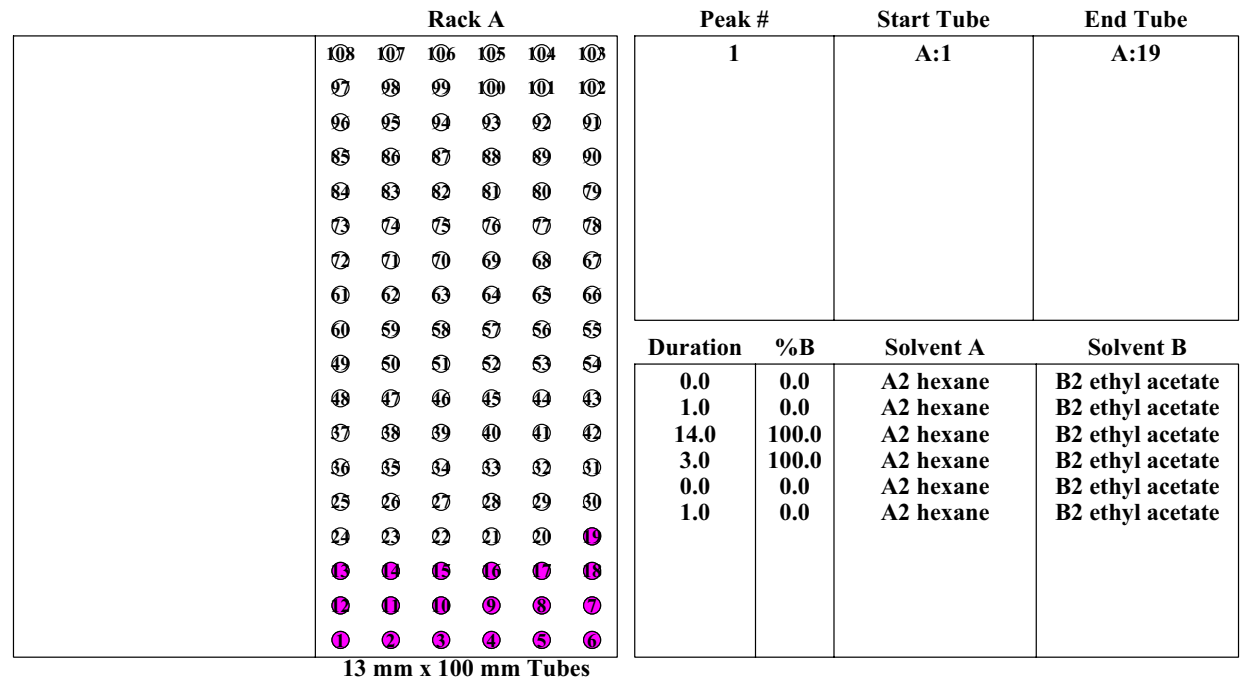

Page 1 of 1 


\section{Synthetic Methods}

\subsubsection{8 (2-(hexyloxy)-5-((pyridin-2-ylmethyl)amino)phenyl)(pyrrolidin-1-}

yl)methanone: G5-111s8

In a $20 \mathrm{~mL}$ vial, (5-amino-2-(hexyloxy)phenyl)(pyrrolidin-1-yl)methanone

(G5-106s7, $1 \mathrm{mmol}, 0.2919 \mathrm{~g})$, and picolinaldehyde (1 mmol, $0.1071 \mathrm{~g}, 0.0951$

$\mathrm{mL})$ were dissolved in methanol $(10 \mathrm{~mL})$. The solution was stirred for 15 minutes, then $\mathrm{NaBH}_{3} \mathrm{CN}(1.59 \mathrm{mmol}, 0.10 \mathrm{~g})$ was added, and the reaction stirred for another 18 hours. The reaction was worked-up by adding $1 \mathrm{M} \mathrm{NaOH}(3 \mathrm{~mL})$, then extracted with ethyl acetate (EtOAc, 2 x $5 \mathrm{~mL}$ ), and finally dried with $\mathrm{MgSO}_{4}$. After filtering, silica gel ( $\sim 2 \mathrm{~g})$ was added to the filtrate. The mixture was concentrated by rotatory evaporation to a powder, which was used as a dry-load for by silica chromatography (FIGURE 7.23). Fractions 14-45 were combined, using dichloromethane to aid the transfer, and concentrated by rotatory evaporation.

${ }^{1} \mathrm{H}-\mathrm{NMR} \delta(\mathrm{ppm})\left(\mathrm{DMSO}_{6}\right): 8.52(1 \mathrm{H}, \mathrm{ddd}, \mathrm{J}=4.82,1.72,0.90 \mathrm{~Hz}), 7.73(1$ $\mathrm{H}, \mathrm{td}, \mathrm{J}=7.66,1.83 \mathrm{~Hz}), 7.36(1 \mathrm{H}, \mathrm{d}, \mathrm{J}=7.86 \mathrm{~Hz}), 7.22-7.27(1 \mathrm{H}, \mathrm{m}), 6.80(1 \mathrm{H}$, d, J = 8.82 Hz), $6.54(1 \mathrm{H}, \mathrm{dd}, \mathrm{J}=8.80,2.95 \mathrm{~Hz}), 6.40(1 \mathrm{H}, \mathrm{d}, \mathrm{J}=2.89 \mathrm{~Hz}), 6.08(1$ $\mathrm{H}, \mathrm{t}, \mathrm{J}=6.16 \mathrm{~Hz}), 4.31(2 \mathrm{H}, \mathrm{d}, \mathrm{J}=6.12 \mathrm{~Hz}), 3.82(2 \mathrm{H}, \mathrm{t}, \mathrm{J}=6.23 \mathrm{~Hz}), 3.03-3.09$ (2 H, m), 1.70-1.87 (4 H, m), 1.54-1.63 (2 H, m), 1.29-1.38 (2 H, m), 1.22-1.29 (4 $\mathrm{H}, \mathrm{m}), 0.87(3 \mathrm{H}, \mathrm{t}, \mathrm{J}=6.67 \mathrm{~Hz})$.

${ }^{13} \mathrm{C}-\mathrm{NMR} \delta(\mathrm{ppm})\left(\mathrm{DMSO}_{\mathrm{d}}\right): 166.7,159.8,148.8,145.4,142.7,136.6$, $128.6,121.9,121.1,114.5,113.2,111.3,68.7,49.1,46.8,45.0,30.9,28.9,25.4$, $25.2,24.1,22.1,13.8$. 


\section{Synthetic Methods}

FIGURE 7.24: Chromatographic purification of G5-111s7.

Sample: grandy5-111s7

RediSep Column: Silica 40g

Flow Rate: $40 \mathrm{ml} / \mathrm{min}$

Equilibration Volume: 5.0 CV

Initial Waste: $0.0 \mathrm{CV}$

Air Purge: $1.0 \mathrm{~min}$

Solvent: A1 ethyl acetate

Solvent: B1 methanol
Rf 200 : OHSU COHEN RF200\#1

Peak Tube Volume: Max.

Non-Peak Tube Volume: Max.

Loading Type: Solid

Wavelength 1 (red): 254nm

Peak Width: 2 min

Threshold: $0.05 \mathrm{AU}$

Wavelength 2 (purple): 280nm
Friday 09 November 2012 12:41PM

All Wavelength (orange): $200 \mathrm{~nm}-360 \mathrm{~nm}$

Peak Width: 2 min

Threshold: 0.20 AU

Run Notes: self-packed column

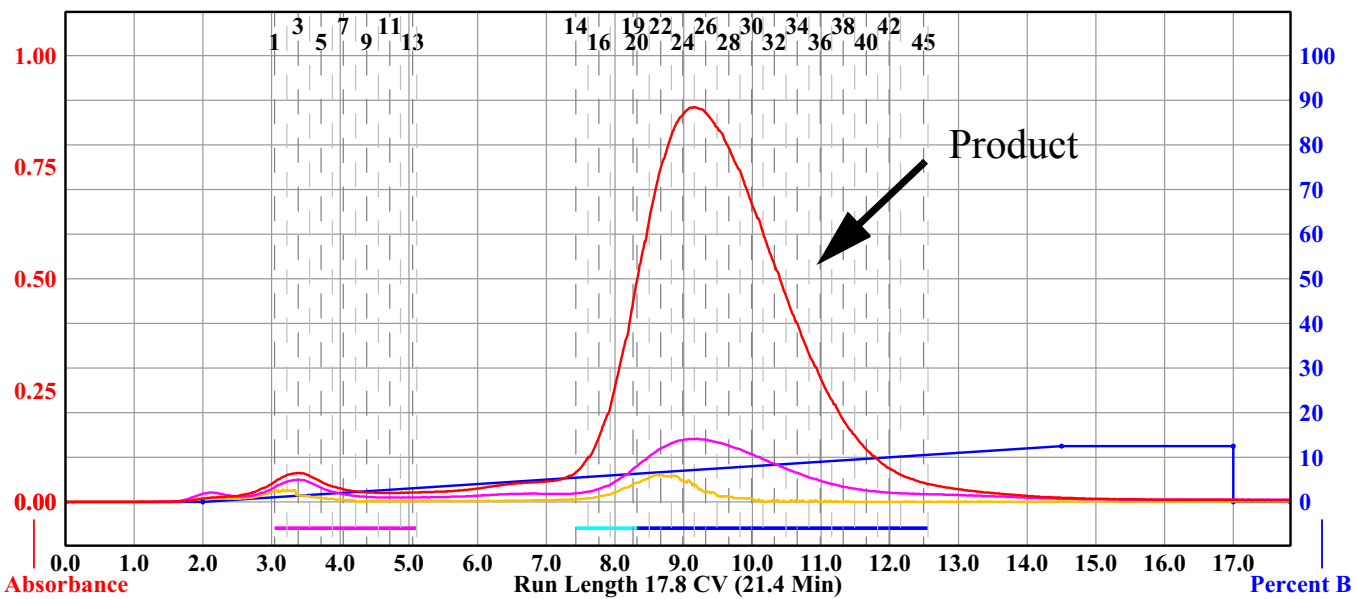

Rack A

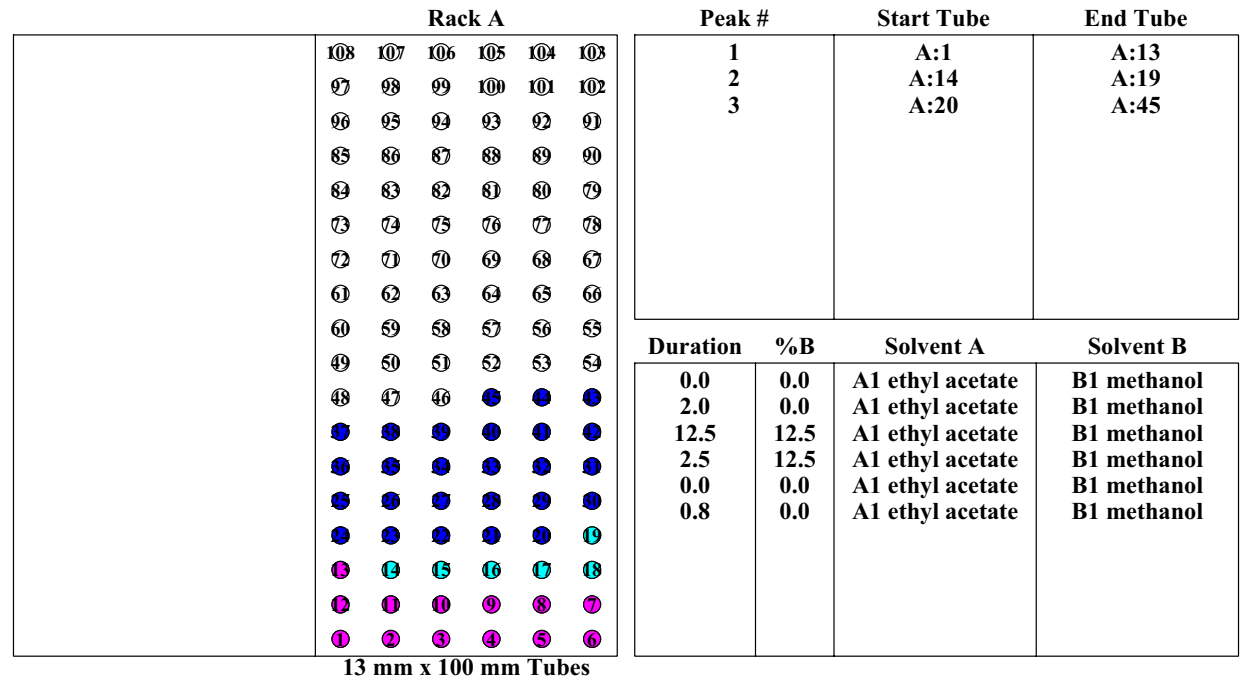

Page 1 of 1 


\section{Synthetic Methods}

\subsubsection{Hexyl 5-amino-2-(hexyloxy)benzoate: G5-048s10}

In a $75 \mathrm{~mL} \# 25$ bushing pressure vessel (ChemGlass: CG-1880-43 with CG364-62) connected to a Parr hydrogenator, a solution of ethyl acetate (EtOAc, 30 $\mathrm{mL})$ and glacial acetic acid (60 mmol, $3.603 \mathrm{~g}, 3.43 \mathrm{~mL})$ was added to hexyl 2(hexyloxy)-5-nitrobenzoate (G5-047s10, $29.1 \mathrm{mmol}, 10.23 \mathrm{~g}$ ) and 10\% $\mathrm{Pd} / \mathrm{C}$ $(0.25 \mathrm{~g})$. The vessel was sealed, then evacuated and filled with $\mathrm{H}_{2}$ to 55 PSI three times. The reaction mixture was stirred until the pressure ceased to drop. The mixture was filtered through a packed plug of celite $(\sim 5 \mathrm{~cm}$ thick $)$, and the plug washed with EtOAc $(\sim 30 \mathrm{~mL})$ and water $(\sim 20 \mathrm{~mL})$. Water $(200 \mathrm{~mL})$ was added to the filtrate, and the $\mathrm{pH}$ was adjusted with $\mathrm{NaHCO}_{3}$ until the $\mathrm{pH}=8$. After mixing throughly, the phases were separated, and the aqueous phase was extracted with EtOAc $(2 \times 50 \mathrm{~mL})$. The combined EtOAc phases were washed with brine (100 $\mathrm{mL}$ ), then dried with $\mathrm{MgSO}_{4}$. After filtering, the filtrate was concentrated by rotatory evaporation to an oil. A ${ }^{1} \mathrm{H}-\mathrm{NMR}$ spectrum of the oil revealed that it was 82.95 mass \% product with the remainder EtOAc.

Note: Later experiments revealed that the use of acid was unnecessary.

${ }^{1} \mathrm{H}-\mathrm{NMR} \delta(\mathrm{ppm})\left(\mathrm{DMSO}_{6}\right): 6.85(1 \mathrm{H}, \mathrm{d}, \mathrm{J}=2.88 \mathrm{~Hz}), 6.83(1 \mathrm{H}, \mathrm{d}, \mathrm{J}=8.76$ Hz), $6.70(1 \mathrm{H}, \mathrm{dd}, \mathrm{J}=8.73,2.89 \mathrm{~Hz}), 4.86(2 \mathrm{H}, \mathrm{s}), 4.16(2 \mathrm{H}, \mathrm{t}, \mathrm{J}=6.52 \mathrm{~Hz}), 3.84$ (2 H, t, J = 6.34 Hz), 1.59-1.70 (4 H, m), 1.34-1.45 (4 H, m), 1.22-1.34 (8 H, m), 0.82-0.93 (6 H, m). 


\section{Synthetic Methods}

7.1.2.30 (5-amino-2-(hexyloxy)phenyl)(pyrrolidin-1-yl)methanone : G5-106s 7

In a $75 \mathrm{~mL} \# 25$ bushing pressure vessel (ChemGlass: CG-1880-43 with CG-

364-62) connected to a Parr hydrogenator, ethanol (30 mL) was added to (2-(hexyloxy)-5-nitrophenyl)(pyrrolidin-1-yl)methanone (G5-099s8, 7.27 mmol, 2.3298

g) and $10 \% \mathrm{Pd} / \mathrm{C}(0.2387 \mathrm{~g})$. The vessel was sealed, then evacuated and filled with $\mathrm{H}_{2}$ to 55 PSI three times. The reaction mixture was stirred until the pressure ceased to drop. The mixture was filtered through a packed plug of celite ( $\sim 2 \mathrm{~cm}$ thick), and the plug washed with ethanol $(\sim 10 \mathrm{~mL})$. The filtrate was concentrated by rotatory evaporation to an oil. The oil was dissolved in ethyl acetate (EtOAc, $10 \mathrm{~mL}$ ), and the solution was extracted with water $(2 \times 5 \mathrm{~mL})$. The EtOAc phase was dried with $\mathrm{MgSO}_{4}$. After filtering, the filtrate was concentrated by rotatory evaporation to an oil. A ${ }^{1} \mathrm{H}-\mathrm{NMR}$ spectrum of the oil revealed that it was 86.83 mass $\%$ product with the remainder EtOAc (2.1567 g gross, $1.8727 \mathrm{~g}$ net product, $88.7 \%$ yield).

${ }^{1} \mathrm{H}-\mathrm{NMR} \delta(\mathrm{ppm})\left(\mathrm{DMSO}_{\mathrm{d}}\right): 6.75(1 \mathrm{H}, \mathrm{d}, \mathrm{J}=8.70 \mathrm{~Hz}), 6.54(1 \mathrm{H}, \mathrm{dd}, \mathrm{J}=$ 8.68, $2.80 \mathrm{~Hz}), 6.38(1 \mathrm{H}, \mathrm{d}, \mathrm{J}=2.78 \mathrm{~Hz}), 4.74(2 \mathrm{H}, \mathrm{s}), 3.82(2 \mathrm{H}, \mathrm{t}, \mathrm{J}=6.23 \mathrm{~Hz})$, $3.39(2 \mathrm{H}, \mathrm{t}, \mathrm{J}=6.88 \mathrm{~Hz}), 3.08-3.17(2 \mathrm{H}, \mathrm{m}), 1.73-1.88(4 \mathrm{H}, \mathrm{m}), 1.55-1.65(2 \mathrm{H}$, m), 1.31-1.40 (2 H, m), 1.24-1.31 (4 H, m), 0.87 (3 H, t, J = 6.72 Hz).

\subsubsection{3-(1,3-dioxolan-2-yl)-4-(hexyloxy)aniline: G5-124s5}

In a $75 \mathrm{~mL} \# 25$ bushing pressure vessel (ChemGlass: CG-1880-43 with CG364-62) connected to a Parr hydrogenator, a solution of ethyl acetate (EtOAc, 20 $\mathrm{mL})$ and ethanol (5 mL) was added to 2-(2-(hexyloxy)-5-nitrophenyl)-1,3-dioxol- 


\section{Synthetic Methods}

ane (G5-122s6, $5.73 \mathrm{~g}, 19.4 \mathrm{mmol})$ and $10 \% \mathrm{Pd} / \mathrm{C}(0.8 \mathrm{~g})$. The vessel was sealed, then evacuated and filled with $\mathrm{H}_{2}$ to 55 PSI three times. The reaction mixture was stirred until the pressure ceased to drop. The mixture was filtered through a packed plug of celite $(\sim 5 \mathrm{~cm}$ thick), and the plug washed with EtOAc $(\sim 20 \mathrm{~mL})$. The filtrate was concentrated by rotatory evaporation to an oil. A ${ }^{1} \mathrm{H}-\mathrm{NMR}$ spectrum of the oil revealed that it was 90.0 mass\% product with the remainder EtOAc $(5.28 \mathrm{~g}$ gross, 4.752 g net product, $92.3 \%$ ).

${ }^{1} \mathrm{H}-\mathrm{NMR} \delta(\mathrm{ppm})\left(\right.$ DMSO-d $\left._{6}\right): 6.72(1 \mathrm{H}, \mathrm{d}, \mathrm{J}=8.64 \mathrm{~Hz}), 6.69(1 \mathrm{H}, \mathrm{d}, \mathrm{J}=2.84$ Hz), $6.52(1 \mathrm{H}, \mathrm{dd}, \mathrm{J}=8.60,2.89 \mathrm{~Hz}), 5.89(1 \mathrm{H}, \mathrm{s}), 4.68(2 \mathrm{H}, \mathrm{s}), 3.86-4.07$ (4 H, m), 3.83 (2 H, m), 1.60-1.69 (2 H, m), 1.33-1.44 (2 H, m), 1.26-1.33 (4 H, m), 0.88 (3 H, t, J = 6.58 Hz).

${ }^{13}$ C-NMR $\delta(p p m)\left(\right.$ DMSO-d $\left._{6}\right): 148.2,142.2,126.6,115.4,114.4,113.3,112.7$, $98.3,69.1,31.0,30.9,29.0,28.9,25.3,25.2,22.1,16.0,13.9$ (2 C).

\subsubsection{2-(5-bromo-2-(hexyloxy)phenyl)-2-phenylacetonitrile: G4-026s10}

A $20 \mathrm{~mL}$ vial was charged with $\operatorname{InBr}_{3}(0.0117 \mathrm{~g}, 0.033 \mathrm{mmol})$, evacuated and filled with $\mathrm{N}_{2}$ followed by the addition of anhydrous dichloromethane (DCM, 2.5 $\mathrm{mL}$ ) and trimethylsilylcyanide (TMSCN, $0.069 \mathrm{~mL}, 54.5 \mathrm{mg}, 0.550 \mathrm{mmol})$. In a round bottom flask, and under $\mathrm{N}_{2}$ atmosphere, (5-bromo-2-(hexyloxy)phenyl)(phenyl)methanol (G4-019s 14, $100 \mathrm{mg}, 0.275 \mathrm{mmol}$ ) was dissolved in anhydrous DCM (2.5 mL). The solution containing G4-019s14 was added dropwise to 


\section{Synthetic Methods}

the $\mathrm{InBr}_{3} / \mathrm{TMSCN}$ solution at a rate of 2-3 drops per second to ensure no color change occurred, which is an indication of a side reaction that results in the transfer of the n-hexyl group from the phenolic oxygen to the methanolic oxygen. After the addition of G4-019s14 was complete, the reaction was stirred for an additional 30 minutes. Basic alumina ( $\sim 1 \mathrm{~g})$ was added to the reaction mixture, then the solvent was removed by rotatory evaporation. The resulting powder was used as a dry load for basic alumina chromatography (FIGURE 7.25). Fraction 11 was concentrated by rotatory evaporation to a colorless oil that slowly crystallized to a white solid (0.0749 g, 73.2\% yield).

Notes: Using silica, instead of basic alumina, as the stationary phase during chromatograpghy results in an impure product. Also, the use of a gradient is required to remove residual TMSCN (UV inactive at $254 \mathrm{~nm}$ and $280 \mathrm{~nm}$ ).

${ }^{1} \mathrm{H}_{-N M R} \delta(\mathrm{ppm})\left(\mathrm{DMSO}_{6}\right):$ 7.50-7.65 (2 H, m), 7.30-7.42 (5 H, m), $7.03(1$ H, d, J = 8.41 Hz), $5.77(1 \mathrm{H}, \mathrm{s}), 3.90-4.00(2 \mathrm{H}, \mathrm{m}), 1.59-1.68(2 \mathrm{H}, \mathrm{m}), 1.21-1.36$ (6 H, m), 084-0.90 (3 H, m).

${ }^{13} \mathrm{C}-\mathrm{NMR} \delta(\mathrm{ppm})\left(\mathrm{DMSO}_{6}\right): 155.0,135.1,132.5,131.3,128.8,127.9$, $127.4,126.4,119.4,114.7,111.6,68.3,36.4,30.9,28.3,25.0,22.0,13.9$.

HRMS (ESI) $m / z$ for $\mathrm{C}_{20} \mathrm{H}_{22} \mathrm{BrNNaO}^{+}[\mathrm{M}+\mathrm{Na}]^{+}$: calcd, 394.07770; found, 394.07787. 


\section{Synthetic Methods}

FIGURE 7.25: Chromatographic purification of G4-026s8.

Sample: grandy4-026s8

RediSep Column: Al2O3 pH=7 24g SN: E0410597B6D1A Lot: 2115308090W

Flow Rate: $30 \mathrm{ml} / \mathrm{min}$

Equilibration Volume: 3.0 CV

Initial Waste: $0.0 \mathrm{CV}$

Air Purge: $0.5 \mathrm{~min}$

Solvent: A1 hexane

Solvent: B1 ethyl acetate
Rf 200 : OHSU COHEN RF200\#1

Tuesday 14 February 2012 04:48PM

Peak Tube Volume: Max.

Non-Peak Tube Volume: Max.

Loading Type: Liquid

Wavelength 1 (red): 254nm

Peak Width: 1 min

Threshold: 0.05 AU

Wavelength 2 (purple): 280nm

Run Notes: Basic Alumina was used.

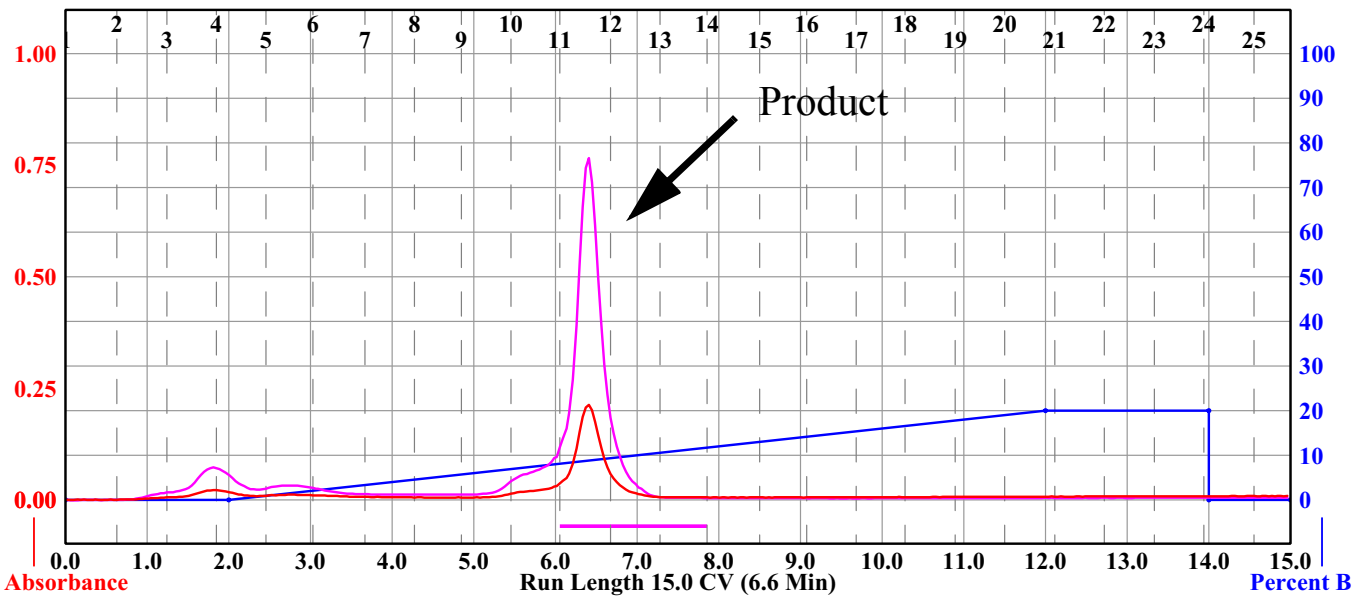

Rack A

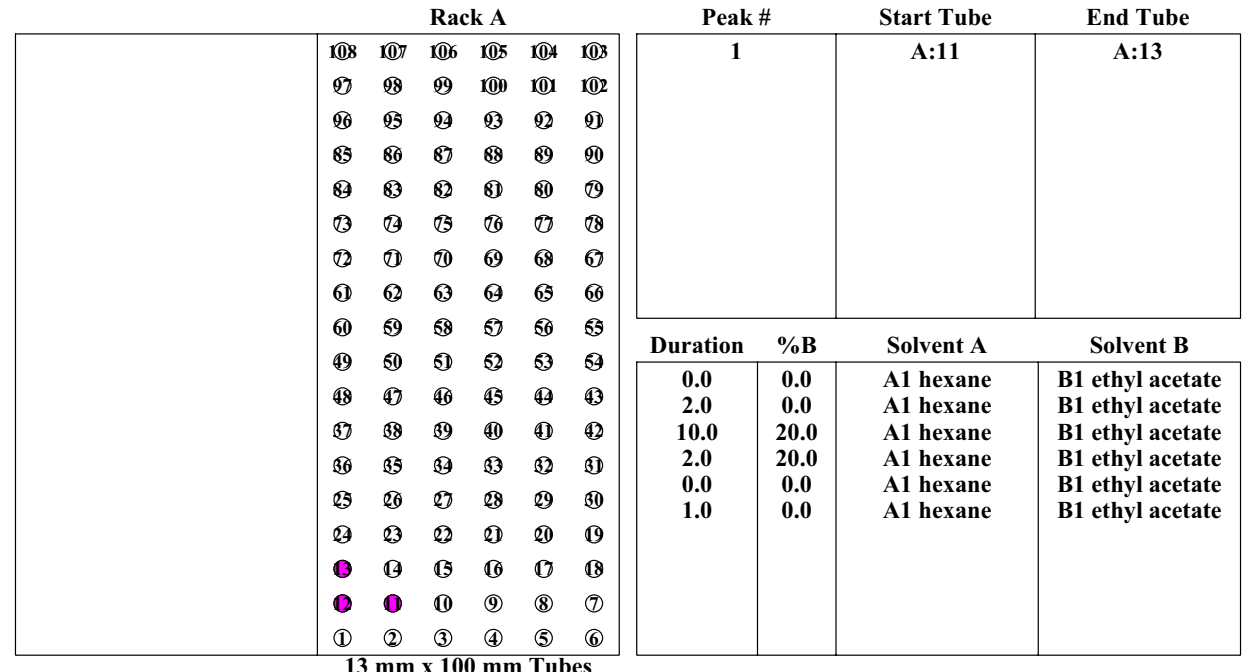

Page 1 of 1 


\section{Synthetic Methods}

\subsubsection{2-(5-(4-fluorophenoxy)-2-(hexyloxy)phenyl)-2-phenylacetonitrile: G4-}

079s9

A round bottom flask was charged with $\operatorname{InBr}_{3}(0.1496 \mathrm{~g}, 0.422 \mathrm{mmol})$, evacuated and filled with $\mathrm{N}_{2}$, followed by the addition of anhydrous dichloromethane (DCM, $35 \mathrm{~mL}$ ) and trimethylsilylcyanide (TMSCN, $1.056 \mathrm{~mL}, 0.837 \mathrm{~g}, 8.44$ mmol). In a separate round bottom flask, and under $\mathrm{N}_{2}$ atmosphere, (5-(4-fluorophenoxy)-2-(hexyloxy)phenyl)(phenyl)methanol (G4-074s14, 1.6659 g, 4.22 mmol) was dissolved in anhydrous DCM (35 mL). The solution containing G4074s14, was added dropwise to the $\operatorname{InBr}_{3} / \mathrm{TMSCN}$ solution at a rate of 2-3 drops per second to ensure no color change occurred, which is an indication of a side reaction that results in the transfer of the n-Hexyl group from the phenolic oxygen to the methanolic oxygen. Towards the end of the reaction, additional TMSCN $(0.5$ $\mathrm{mL}, 0.3965 \mathrm{~g}, 4.00 \mathrm{mmol}$ ) was added because a color change was occurring despite the slow rate of addition of G4-074s14. After the addition of G4-074s14 was complete, the reaction was stirred for an additional 30 minutes. Basic alumina ( $\sim 5 \mathrm{~g}$ ) was added to the reaction mixture, then the solvent was removed by rotatory evaporation. The resulting powder was used as a dry load for basic alumina chromatography (see FIGURE 7.26). Fractions 1-51 were combined and concentrated by rotatory evaporation to a pale yellow oil (1.7027 $\mathrm{g}, 88.5 \%$ yield).

Notes: Using silica, instead of basic alumina, as the stationary phase during chromatograpghy results in an impure product (mixture of regenerated starting material (G4-074s14), the desired product and a side-product: 4-(4-fluorophe- 


\section{Synthetic Methods}

noxy)-2-((hexyloxy)(phenyl)methyl)phenol). Also, the use of a gradient is

required to remove residual TMSCN (UV inactive at $254 \mathrm{~nm}$ and $280 \mathrm{~nm}$ ).

${ }^{1} \mathrm{H}-\mathrm{NMR} \delta(\mathrm{ppm})\left(\mathrm{DMSO}_{6}\right): 7.30$ - $7.41(\mathrm{~m}, 5 \mathrm{H}), 7.19$ - $7.24(\mathrm{~m}, 2 \mathrm{H}), 7.094$ $(\mathrm{d}, \mathrm{J}=3.0 \mathrm{~Hz}, 1 \mathrm{H}), 7.060(\mathrm{~d}, \mathrm{~J}=9.0 \mathrm{~Hz}, 1 \mathrm{H}), 7.04-7.005(\mathrm{~m}, 2 \mathrm{H}), 6.994(\mathrm{dd}, \mathrm{J}=$ $9.0 \mathrm{~Hz}, 3.0 \mathrm{~Hz}, 1 \mathrm{H}), 5.748$ (s, 1H), 3.90 - 3.96 (m, 2H), 1.61-1.67 (m, 2H), 1.30 $1.36(\mathrm{~m}, 2 \mathrm{H}), 1.22-1.30(\mathrm{~m}, 4 \mathrm{H}), 0.874(\mathrm{t}, \mathrm{J}=7.5 \mathrm{~Hz}, 3 \mathrm{H})$.

${ }^{13} \mathrm{C}-\mathrm{NMR} \delta(\mathrm{ppm})\left(\mathrm{DMSO}_{\mathrm{d}}\right): 157.88(\mathrm{~d}, \mathrm{~J}=239.9 \mathrm{~Hz}), 153.47(\mathrm{~d} . \mathrm{J}=1.5$ Hz), 151.86, 149.74, 135.37, 128.76, 127.75, 127.41, 125.37, 119.79, 119.76, 119.62. $119.53(d, J=7.5 \mathrm{~Hz}), 116.47$ (d, $J=22.6 \mathrm{~Hz}), 113.67,68.33,36.61$, $30.94,28.50,25.04,21.99,13.86$.

${ }^{19} \mathrm{~F}-\mathrm{NMR} \delta(\mathrm{ppm})\left(\mathrm{DMSO}-\mathrm{d}_{6}\right.$, with $\mathrm{C}_{6} \mathrm{~F}_{6}$ as reference at $\left.-164.9 \mathrm{ppm}\right):-122.84$ to -122.94 .

HRMS (ESI) $m / z$ for $\mathrm{C}_{26} \mathrm{H}_{26} \mathrm{FNNaO}_{2}{ }^{+}[\mathrm{M}+\mathrm{Na}]^{+}$: calcd, 426.18398; found, 426.18435. 


\section{Synthetic Methods}

FIGURE 7.26: Chromatographic purification of G4-079s8.

Sample: grandy4-079s8

RediSep Column: Al2O3 pH=7 160g

Flow Rate: $60 \mathrm{ml} / \mathrm{min}$

Equilibration Volume: 3.0 CV

Initial Waste: $0.0 \mathrm{CV}$

Air Purge: $1.0 \mathrm{~min}$

Solvent: A1 hexane

Solvent: B1 ethyl acetate
Rf 200 : OHSU COHEN RF200\#1

Thursday 19 April 2012 01:55PM

Peak Tube Volume: Max.

Non-Peak Tube Volume: Max.

Loading Type: Solid

Wavelength 1 (red): 254nm

Peak Width: 2 min

Threshold: $0.05 \mathrm{AU}$

Wavelength 2 (purple): $280 \mathrm{~nm}$

Run Notes: Basic Alumina was used.

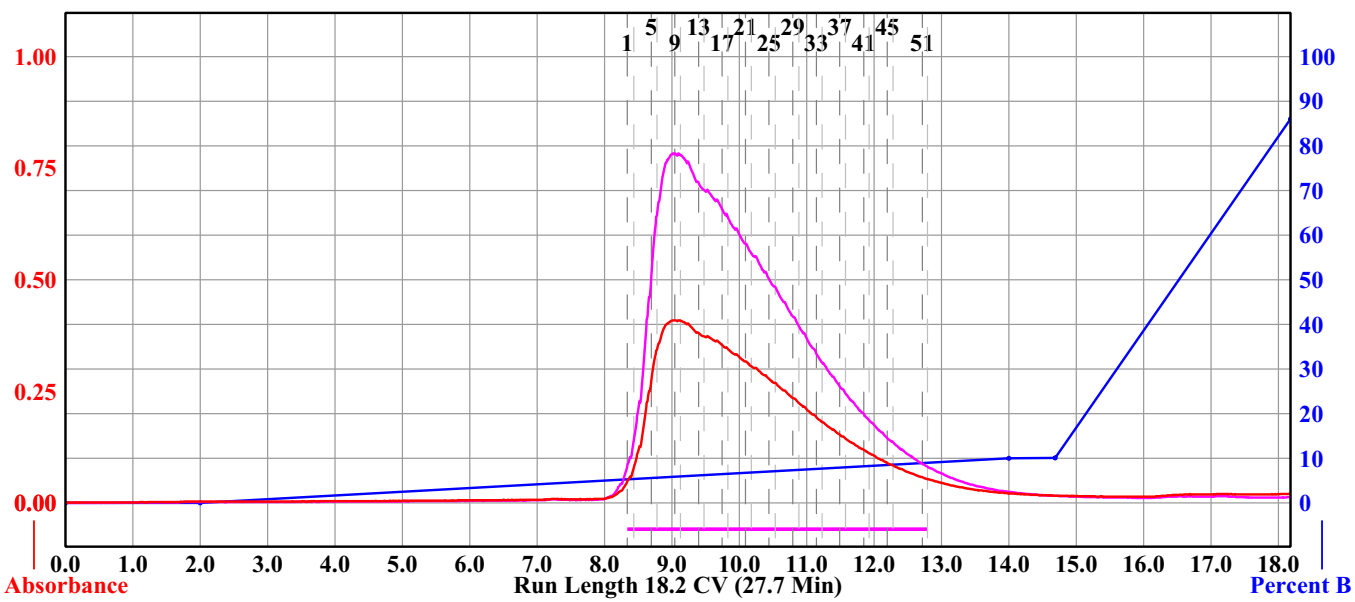

Rack A

\begin{tabular}{|c|c|c|c|c|c|}
\hline 108 & (107 & 106 & 105 & (144) & (103 \\
\hline (2) & $\mathscr{0}$ & $\mathscr{9 9}$ & (100 & (10) & (102 \\
\hline (ன & 98 & (2) & (23) & (2) & (21) \\
\hline 87 & 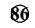 & 87 & 88 & 89 & (20 \\
\hline 8 & 83 & (32) & (11) & (10) & (1) \\
\hline$(3)$ & (8) & 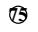 & (2) & (27) & 88 \\
\hline (12) & (11) & (11) & 69 & 68 & (6) \\
\hline (1) & (2) & 63 & 6 & 67 & ஐ \\
\hline (1) & 59 & 68 & 57 & 60 & 65 \\
\hline (4) & (1) & 6 & 62 & 63 & 69 \\
\hline (48) & (7) & (40) & (45) & (4) & (4) \\
\hline (B) & $B$ & (3) & (4) & (4) & (4) \\
\hline (6) & (3) & (3) & (B) & (2) & (1) \\
\hline (2) & 6 & 0 & 8 & 0 & (B) \\
\hline 8 & (2) & (2) & (1) & (2) & (1) \\
\hline (1) & $\theta$ & (1) & (6) & 0 & 0 \\
\hline 4 & (1) & (1) & (2) & (8) & (1) \\
\hline (1) & (2) & (3) & (4) & (5) & (6) \\
\hline
\end{tabular}

$13 \mathrm{~mm} \times 100 \mathrm{~mm}$ Tubes

\begin{tabular}{|c|c|c|c|}
\hline \multicolumn{2}{|c|}{ Peak \# } & Start Tube & End Tube \\
\hline \multicolumn{2}{|l|}{1} & \multirow[b]{2}{*}{ Solvent A } & \multirow[t]{2}{*}{ A:51 } \\
\hline Duration & $\%$ B & & \\
\hline 0.0 & 0.0 & A1 hexane & B1 ethyl acetate \\
\hline 2.0 & 0.0 & A1 hexane & B1 ethyl acetate \\
\hline 12.0 & 10.0 & A1 hexane & B1 ethyl acetate \\
\hline 0.7 & 10.1 & A1 hexane & B1 ethyl acetate \\
\hline 3.5 & 86.0 & A1 hexane & B1 ethyl acetate \\
\hline & & & \\
\hline & & & \\
\hline & & & \\
\hline
\end{tabular}

Page 1 of 1 


\section{Synthetic Methods}

\subsubsection{2-(5-bromo-2-(hexyloxy)phenyl)-1,3-dioxolane: G1-130s5}

In a round bottom flask, equipped with a Dean-Stark trap and a reflux condenser, 5-bromo-2-(hexyloxy)benzaldehyde (25 mmol, $7.130 \mathrm{~g})$, ethylene glycol (50 mmol, $3.105 \mathrm{~g}, 2.790 \mathrm{~mL})$ and p-toluenesulfonic acid monohydrate $(0.25$ mmol, $0.0475 \mathrm{~g})$ were dissolve in toluene $(100 \mathrm{~mL})$. The reaction mixture was refluxed at $150{ }^{\circ} \mathrm{C}$ (pot temperature) for 18 hours. The reaction mixture was extracted with $0.1 \mathrm{M} \mathrm{Na}_{2} \mathrm{CO}_{3}(3 \times 100 \mathrm{~mL})$, and the toluene phase was dried with $\mathrm{MgSO}_{4}$. After filtering, the filtrate was concentrated by rotatory evaporation to an oil that smelled of toluene $(8.3989 \mathrm{~g})$.

${ }^{1} \mathrm{H}-\mathrm{NMR} \delta(\mathrm{ppm})\left(\mathrm{CH}_{2} \mathrm{Cl}_{2}-\mathrm{d}_{2}\right): 7.64(1 \mathrm{H}, \mathrm{d}, \mathrm{J}=2.62 \mathrm{~Hz}), 7.44(1 \mathrm{H}, \mathrm{dd}, \mathrm{J}=$ 8.76, 2.63 Hz), 6.83 (1 H, d, J = 8.78 Hz), $6.11(1 \mathrm{H}, \mathrm{s}), 3.98-4.18$ (6 H, m), 1.78$1.87(2 \mathrm{H}, \mathrm{m}), 1.45-1.55(2 \mathrm{H}, \mathrm{m}), 1.35-1.44(4 \mathrm{H}, \mathrm{m}), 0.96(3 \mathrm{H}, \mathrm{t}, \mathrm{J}=6.66 \mathrm{~Hz})$.

\subsubsection{2-(2-(hexyloxy)-5-nitrophenyl)-1,3-dioxolane: G5-122s6}

In a round bottom flask, equipped with a Dean-Stark trap and a reflux condenser, 2-(hexyloxy)-5-nitrobenzaldehyde (G5-120s11, $8.00 \mathrm{~g}, 31.57 \mathrm{mmol}$ ), ethylene glycol (35 mmol, $2.1725 \mathrm{~g}, 1.95 \mathrm{~mL}$ ) and p-toluenesulfonic acid monohydrate $(0.0475 \mathrm{~g})$ were dissolve in toluene $(50 \mathrm{~mL})$. The reaction mixture was refluxed at $150^{\circ} \mathrm{C}$ (pot temperature) for 48 hours. Basic alumina $(\sim 5 \mathrm{~g})$ was added to the reaction mixture, which was then concentrated by rotatory evaporation to a paste. The paste was used as a dry-load for basic alumina chromatography 


\section{Synthetic Methods}

(FIGURE 7.27). Fractions 19-87 were combined, and concentrated by rotatory

evaporation $(5.73 \mathrm{~g})$.

${ }^{1} \mathrm{H}-\mathrm{NMR} \delta(\mathrm{ppm})\left(\mathrm{DMSO}_{\mathrm{d}}\right): 8.27(1 \mathrm{H}, \mathrm{dd}, \mathrm{J}=9.08,3.00 \mathrm{~Hz}), 8.22(1 \mathrm{H}, \mathrm{d}, \mathrm{J}$

$=2.92 \mathrm{~Hz}), 7.28(1 \mathrm{H}, \mathrm{d}, \mathrm{J}=9.14 \mathrm{~Hz}), 6.01(1 \mathrm{H}, \mathrm{s}), 4.18(2 \mathrm{H}, \mathrm{t}, \mathrm{J}=6.41 \mathrm{~Hz})$,

3.93-4.15 (4 H, m), 1.71-1.80 (2 H, m), 1.38-1.49 (2 H, m), 1.26-1.38 (4 H, m),

$0.88(3 \mathrm{H}, \mathrm{t}, \mathrm{J}=6.75 \mathrm{~Hz})$.

${ }^{13}$ C-NMR $\delta(p p m)\left(\right.$ DMSO-d $\left._{6}\right): 162.0,140.2,127.0,126.6,122.4,112.7,97.4$, 69.0, 64.9, 30.8, 28.2, 24.9, 22.0, 13.8. 


\section{Synthetic Methods}

FIGURE 7.27: Chromatographic purification of G5-122s5.

Sample: grandy5-122s5

RediSep Column: Al2O3 $\mathrm{pH}=\mathbf{7 4 8 g}$

Flow Rate: $50 \mathrm{ml} / \mathrm{min}$

Equilibration Volume: $5.0 \mathrm{CV}$

Initial Waste: $0.0 \mathrm{CV}$

Air Purge: $1.0 \mathrm{~min}$

Solvent: A2 hexane

Solvent: B2 dichloromethane
Rf 200 : OHSU COHEN RF200\#1

Peak Tube Volume: Max.

Non-Peak Tube Volume: Max.

Loading Type: Solid

Wavelength 1 (red): 254nm

Peak Width: 1 min

Threshold: 0.05 AU

Wavelength 2 (purple): 280nm
Friday 19 October 2012 10:38AM

All Wavelength (orange): $200 \mathrm{~nm}$ - 360nm

Peak Width: 1 min

Threshold: 0.20 AU

Run Notes: self-packed basic alumina

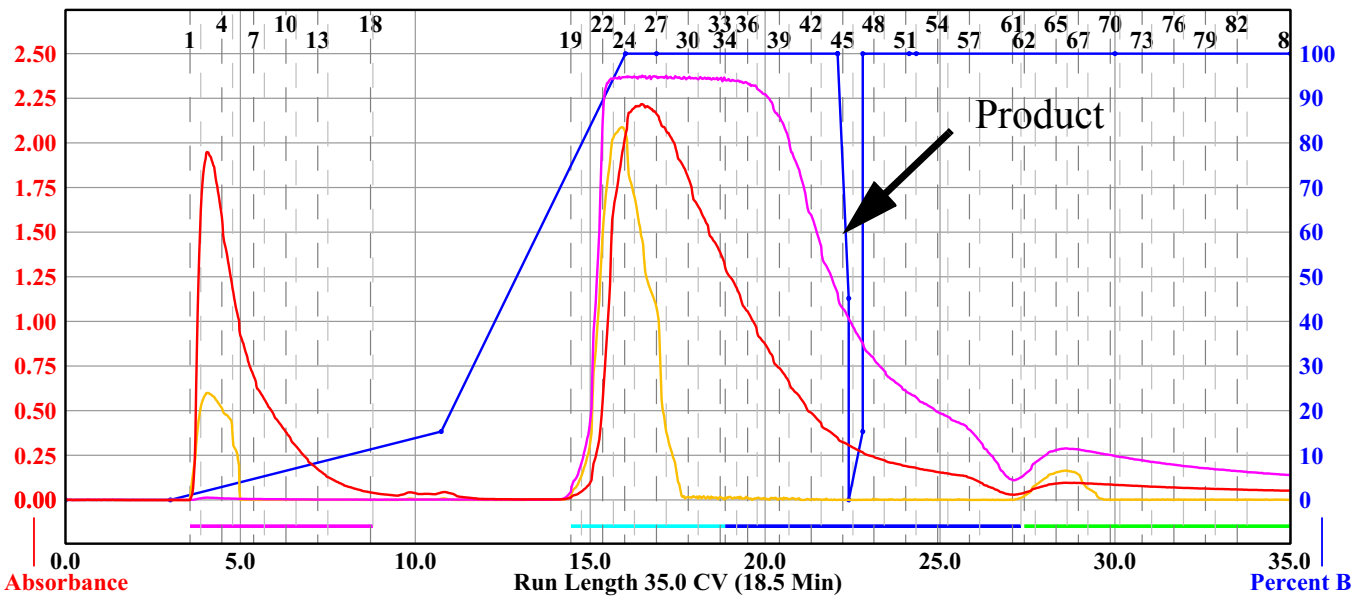

Rack A

\begin{tabular}{|c|c|c|c|c|c|}
\hline 108 & (1)7 & 106 & 105 & (104) & (10) \\
\hline (2) & 08 & (9) & 100 & (10) & (102 \\
\hline னூ & (25) & (2) & (2) & (2) & (21) \\
\hline 8 & 80 & 87 & 88 & 89 & פD \\
\hline 8 & 83 & (2) & (1) & (810 & (1) \\
\hline 13 & (1) & (2) & (20 & (1) & 88 \\
\hline (12) & (1) & (1) & (9) & 68 & (2) \\
\hline 5 & (2) & 6 & 6 & 65 & ஏ \\
\hline 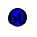 & ( & - & 0 & (1) & ๑ \\
\hline & 0 & ? & ? & 2 & \\
\hline & 2 & 6 & 8 & 0 & \\
\hline (1) & - & - & B3 & (B2) & (11) \\
\hline (2) & 6 & 87 & 8 & (29) & (10) \\
\hline (2) & 23 & (2) & (21) & (21) & (1) \\
\hline 10 & 0 & (1) & 0 & 0 & (1) \\
\hline (1) & (1) & (1) & (2) & (8) & (1) \\
\hline (1) & (2) & (3) & (4) & (5) & (6) \\
\hline
\end{tabular}

$13 \mathrm{~mm} \times 100 \mathrm{~mm}$ Tubes

\begin{tabular}{|c|c|c|c|}
\hline \multicolumn{2}{|c|}{ Peak \# } & Start Tube & End Tube \\
\hline $\begin{array}{l}1 \\
2 \\
3 \\
4\end{array}$ & & $\begin{array}{c}\text { A:1 } \\
\mathbf{A}: 19 \\
\mathbf{A}: 34 \\
\mathbf{A}: 62\end{array}$ & $\begin{array}{l}\text { A:18 } \\
\text { A:33 } \\
\text { A:61 } \\
\text { A:87 }\end{array}$ \\
\hline Duration & $\%$ В & Solvent A & Solvent B \\
\hline 0.0 & 0.0 & A2 hexane & B2 dichloromethan \\
\hline 3.0 & 0.0 & A2 hexane & B2 dichloromethan \\
\hline 7.7 & 15.3 & A2 hexane & B2 dichloromethan \\
\hline 5.3 & 100.0 & A2 hexane & B2 dichloromethan \\
\hline 0.9 & 100.0 & A2 hexane & B2 dichloromethan \\
\hline 5.2 & 100.0 & A2 hexane & B2 dichloromethan \\
\hline 0.0 & 100.0 & A2 hexane & B2 dichloromethan \\
\hline 0.3 & 45.2 & A2 hexane & B2 dichloromethan \\
\hline 0.0 & $\mathbf{0 . 0}$ & A2 hexane & B2 dichloromethan \\
\hline 0.4 & 15.3 & A2 hexane & B2 dichloromethan \\
\hline$\cdots$ & $\cdots$ & & \\
\hline
\end{tabular}

Page 1 of 1 


\section{Synthetic Methods}

\subsubsection{2-(5-(4-fluorophenoxy)-2-(hexyloxy)phenyl)-2-phenylethanamine}

hydrochloride: ET-92 (G4-091s14 and G5-002s12)

In a round bottom flask equipped with a reflux condenser, 2-(5-(4-fluorophenoxy)-2-(hexyloxy)phenyl)-2-phenylacetonitrile (G4-079s9, 0.0574 g, 0.142 mmol) was dissolved in anhydrous tetrahydrofuran (THF, $5 \mathrm{~mL}$ ). The reaction vessel was evacuated and filled with $\mathrm{N}_{2}$, then $1 \mathrm{M} \mathrm{BH}_{3} \cdot \mathrm{DMS}$ in THF $(0.145 \mathrm{~mL}$, $0.145 \mathrm{mmol}$ ) was slowly added by syringe. The reaction was refluxed for 2.5 hours, and then cooled to room temperature. Methanol $(\mathrm{MeOH}, 0.25 \mathrm{~mL}, 6.17$ mmol) was slowly added by syringe. After $\mathrm{H}_{2}$ evolution stopped, $3.0 \mathrm{M} \mathrm{HCl}$ in EtOAc $(0.05 \mathrm{~mL}, 0.15 \mathrm{mmol})$ was added, and the mixture was heated to $40{ }^{\circ} \mathrm{C}$ for 15 minutes. The mixture was then concentrate by rotatory evaporation, chasing with $\mathrm{MeOH}(3 \times 5 \mathrm{~mL})$ and ethyl acetate $(1 \times 2 \mathrm{~mL})$. Next, diethyl ether $(2 \mathrm{~mL})$ was added, and the solution was then cool to $-68^{\circ} \mathrm{C}$. After a precipitate form, the mixture was centrifuged, and the supernatant was decanted off. The white solid was dried under vacuum for 12 hours $(0.0246 \mathrm{~g}, 38.9 \%$ yield $)$.

${ }^{1} \mathrm{H}-\mathrm{NMR} \delta(\mathrm{ppm})\left(\mathrm{DMSO}_{6}\right): 7.895(\mathrm{bs}, 3 \mathrm{H}), 7.30$ - $7.33(\mathrm{~m}, 4 \mathrm{H}), 7.224$ (d, J $=3.0,1 \mathrm{H}), 7.23-7.25(\mathrm{~m}, 1 \mathrm{H}), 7.16-7.21(\mathrm{~m}, 2 \mathrm{H}), 6.96-7.00(\mathrm{~m}, 2 \mathrm{H}), 6.960(\mathrm{~d}$, $\mathrm{J}=9.0 \mathrm{~Hz}, 1 \mathrm{H}), 6.841(\mathrm{dd}, \mathrm{J}=9.0 \mathrm{~Hz}, 3.0 \mathrm{~Hz}, 1 \mathrm{H}), 4.619(\mathrm{t}, \mathrm{J}=8.1 \mathrm{~Hz}, 1 \mathrm{H}), 3.83$ - $3.94(\mathrm{~m}, 2 \mathrm{H}), 3.46$ - $3.54(\mathrm{~m}, 2 \mathrm{H}), 1.66-1.72(\mathrm{~m}, 2 \mathrm{H}), 1.35$ - $1.42(\mathrm{~m}, 2 \mathrm{H}), 1.26-$ $1.35(\mathrm{~m}, 4 \mathrm{H}), 0.891(\mathrm{t}, \mathrm{J}=6.9 \mathrm{~Hz}, 3 \mathrm{H})$. 


\section{Synthetic Methods}

${ }^{13} \mathrm{C}-\mathrm{NMR} \delta(\mathrm{ppm})\left(\mathrm{DMSO}_{\mathrm{d}}\right): 157.61(\mathrm{~d}, \mathrm{~J}=238.4 \mathrm{~Hz}), 154.04(\mathrm{~d} . \mathrm{J}=22.2$

$\mathrm{Hz}), 152.46,149.56,140.32,130.33,128.48,128.03,126.90,119.36,118.92$ (d, J

$=9.1 \mathrm{~Hz}), 118.38,116.29(\mathrm{~d}, \mathrm{~J}=24.1 \mathrm{~Hz}), 113.12,68.07,41.63,42.08,31.00$,

$28.74,25.21,22.04,13.91$.

${ }^{19} \mathrm{~F}-\mathrm{NMR} \delta(\mathrm{ppm})\left(\mathrm{DMSO}-\mathrm{d}_{6}\right.$, with $\mathrm{C}_{6} \mathrm{~F}_{6}$ as reference at $\left.-164.9 \mathrm{ppm}\right):-123.54$ to -123.65 .

HRMS (ESI) $m / z$ for $\mathrm{C}_{26} \mathrm{H}_{31} \mathrm{FNO}_{2}{ }^{+}[\mathrm{M}+\mathrm{H}]^{+}$: calcd, 408.23333; found, 408.23287 .

\subsubsection{7 (2-hydroxy-5-nitrophenyl)(pyrrolidin-1-yl)methanone: G5-098s6 and G5-088s8}

In a round bottom flask, 2-hydroxy-5-nitrobenzoic acid (30 mmol, $5.4956 \mathrm{~g}$ ), and 1-ethyl-3-(3-dimethylaminopropyl)carbodiimide hydrochloride (5.751 g, 30 mmol) were dissolved in anhydrous dichloromethane (DCM, 100mL). The solution was stirred for 5 minutes, then pyrrolidine $(120 \mathrm{mmol}, 8.5544 \mathrm{~g}, 10 \mathrm{~mL})$ was added. The reaction was stirred for 2 hours, then extracted with $0.72 \mathrm{M} \mathrm{HCl}(2 \mathrm{x}$ $56 \mathrm{~mL}$ ) then the $\mathrm{DCM}$ phase was dried with $\mathrm{MgSO}_{4}$. After filtering, the filtrate was concentrated by rotatory evaporator.

${ }^{1} \mathrm{H}-\mathrm{NMR} \delta(\mathrm{ppm})\left(\mathrm{DMSO}_{6}\right): 11.56(1 \mathrm{H}, \mathrm{s}), 8.16(1 \mathrm{H}, \mathrm{dd}, \mathrm{J}=9.06,2.91 \mathrm{~Hz})$, $8.06(1 \mathrm{H}, \mathrm{d}, \mathrm{J}=2.88 \mathrm{~Hz}), 7.07(1 \mathrm{H}, \mathrm{d}, \mathrm{J}=9.07 \mathrm{~Hz}), 3.46(2 \mathrm{H}, \mathrm{t}, \mathrm{J}=6.74 \mathrm{~Hz})$, $3.23(2 \mathrm{H}, \mathrm{t}, \mathrm{J}=6.74 \mathrm{~Hz}), 1.79-1.89(4 \mathrm{H}, \mathrm{m})$. 


\section{Synthetic Methods}

${ }^{13} \mathrm{C}-\mathrm{NMR} \delta(\mathrm{ppm})\left(\right.$ DMSO-d $\left._{6}\right): 164.5,159.7,139.3,126.3,125.9,124.3$, $116.5,47.0,45.4,25.4,23.9$.

\subsubsection{5-(4-fluorophenoxy)-2-(hexyloxy)benzaldehyde: G4-068s20 and G4-70s6} CuI (6.3595 g, $35.01 \mathrm{mmol})$, 1,10-phenanthroline (6.3102 g, $35.02 \mathrm{mmol}), \mathrm{KI}$ $(0.292 \mathrm{~g}, 1.757 \mathrm{mmol})$ and $\mathrm{K}_{2} \mathrm{CO}_{3}(9.6753 \mathrm{~g}, 70.00 \mathrm{mmol})$ were combined in a round bottom flask equipped with a reflux condenser, Dean-Stark trap, addition funnel and gas inlet valve. The apparatus was then purged and filled with $\mathrm{N}_{2}$. Anhydrous dimethylformamide (DMF, $70 \mathrm{~mL}$ ) and toluene $(20 \mathrm{~mL})$ were added. The resulting mixture was stirred for 30 minutes. 5-bromo-2-(hexyloxy)benzaldehyde (G4-012s16, $5.0038 \mathrm{~g}, 17.55 \mathrm{mmol}$ ) was dissolved in anhydrous DMF (10 $\mathrm{mL}$ ), then added to the reaction vessel. Next, 4-fluorophenol (7.8464 g, 69.99 mmol) was dissolved in anhydrous DMF $(20 \mathrm{~mL})$, then transferred to the addition funnel, whereupon one quarter of the solution was added to the reaction. The reaction was heated to $120^{\circ} \mathrm{C}$ for 2 hours. The remaining 4-fluorophenol solution was added dropwise, and heating was continued for a total of 19 hours. The reaction was cooled to room temperature, then water $(500 \mathrm{~mL})$ and hexanes $(250 \mathrm{~mL})$ were added. After mixing and breaking-up the solids, the mixture was filtered through a medium sintered glass funnel. The residue was washed with hexanes $(200 \mathrm{~mL})$ then discarded. The phases of the filtrate were separated. The aqueous phase was extracted with hexanes $(3 \times 250 \mathrm{~mL})$. The combined hexane layers were washed 


\section{Synthetic Methods}

with $0.05 \mathrm{M} \mathrm{NaOH}(2 \times 500 \mathrm{~mL})$, then dried over $\mathrm{MgSO}_{4}$. After filtering, the fil-

trate was concentrated by rotatory evaporator to an oil (5.0759 g).

The crude product was dissolved in dichloromethane and silica gel $(\sim 20 \mathrm{~g})$ was added. The mixture was concentrated by rotatory evaporator to a powder. The powder was used as a dry load for silica chromatography (FIGURE 7.28). Fractions A1-B73 were combined, then concentrated by rotatory evaporator to an oil (3.9806 g, $\sim 75 \%$ desired product by NMR). 


\section{Synthetic Methods}

FIGURE 7.28: Chromatographic purification of G4-068s14.

Sample: grandy4-068s14

RediSep Column: Silica 80g SN: E0410524A7FAB Lot: 2111419020X Flow Rate: $60 \mathrm{ml} / \mathrm{min}$

Equilibration Volume: 3.0 CV

Initial Waste: $0.0 \mathrm{CV}$

Air Purge: $1.0 \mathrm{~min}$

Solvent: A1 hexane

Solvent: B2 dichloromethane
Rf 200 : OHSU COHEN RF200\#1

Thursday 29 March 2012 02:12PM

Peak Tube Volume: Max.

Non-Peak Tube Volume: Max.

Loading Type: Solid (Pause)

Wavelength 1 (red): $254 \mathrm{~nm}$

Peak Width: 2 min

Threshold: 0.05 AU

Wavelength 2 (purple): 280nm

Run Notes:

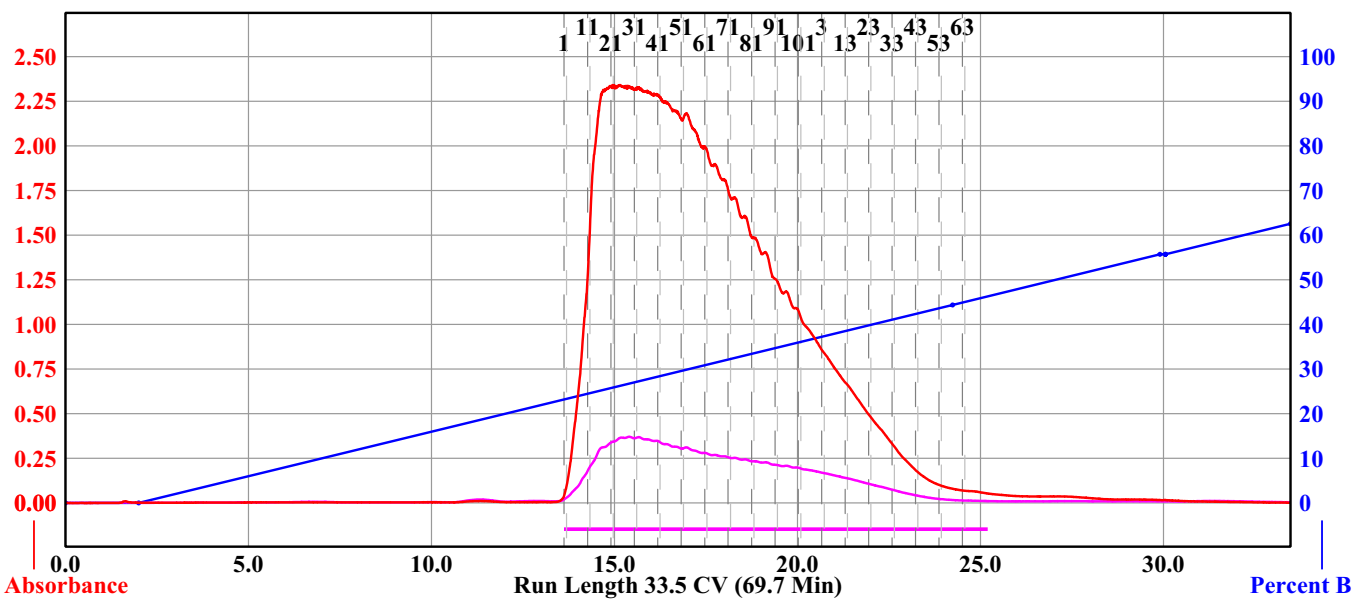

Rack A

\begin{tabular}{|c|c|c|c|c|c|c|c|c|c|c|c|}
\hline 88 & & 106 & 155 & (194 & 03 & 08 & (107) & 106 & 105 & (104 & (103 \\
\hline$n$ & 8 & (2) & 19 & & 102 & & 98 & 99 & & (10) & D) \\
\hline & (2) & (2) & 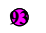 & In & I & V6 & (5) & 9 & & (2) & (1) \\
\hline & 80 & 8 & 8 & 89 & m & 87 & 80 & 87 & 88 & 89 & श) \\
\hline & 8 & (2) & (1) & (11) & 79 & 39 & 83 & 82 & (11) & (10) & (19) \\
\hline & 0 & 8 & (26) & 0 & 88 & (2) & (3) & (1) & (16) & (17) & 18 \\
\hline & (1) & (1) & (9) & (6) & (6) & (12) & (1) & (1) & (9) & 6 & (6) \\
\hline & (2) & 6 & 6 & (6) & 6 & 0 & (2) & 6 & (6) & (6) & (6) \\
\hline & D) & 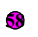 & 5 & 6 & 65 & (1) & 6 & 68 & (5) & 6 & 65 \\
\hline & (10) & E & 62 & 3 & F & (4) & (10) & (1) & (2) & 6 & อ \\
\hline & (4) & (1) & 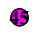 & (4) & $\uplus$ & (48) & 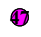 & (4) & (4) & (4) & (1) \\
\hline & (B) & 2 & 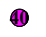 & 4 & 4 & (3) & $B$ & (3) & (41) & (1) & (4) \\
\hline & (B) & (B) & (3) & (3) & (2) & (1) & (3) & (3) & (B) & (3) & (1) \\
\hline & 60 & 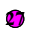 & 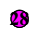 & 6 & (10) & (6) & 0 & 0 & 8 & 29 & (10) \\
\hline & (2) & 22 & (2) & (21) & (1) & (2) & (2) & (2) & (1) & (20) & (1) \\
\hline & 0 & (6) & 0 & 0 & 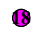 & (1) & 0 & 0 & (6) & 0 & (1) \\
\hline & (1) & (1) & (2) & (8) & (1) & (1) & (1) & (1) & (9) & (8) & (1) \\
\hline & (2) & (3) & (4) & (5) & (6) & (1) & (2) & (3) & (4) & (5) & (6) \\
\hline
\end{tabular}

$13 \mathrm{~mm} \times 100 \mathrm{~mm}$ Tubes $13 \mathrm{~mm} \times 100 \mathrm{~mm}$ Tubes

\begin{tabular}{|c|c|c|c|}
\hline \multicolumn{2}{|c|}{ Peak \# } & Start Tube & End Tube \\
\hline \multicolumn{2}{|l|}{1} & A:1 & B:73 \\
\hline Duration & $\%$ B & Solvent A & Solvent B \\
\hline 0.0 & 0.0 & A1 hexane & B2 dichloromethan \\
\hline 2.0 & 0.0 & A1 hexane & B2 dichloromethan \\
\hline 22.2 & 44.4 & A1 hexane & B2 dichloromethan \\
\hline 5.7 & 55.7 & A1 hexane & B2 dichloromethan \\
\hline 0.1 & 55.7 & A1 hexane & B2 dichloromethan \\
\hline 0.0 & 55.7 & A1 hexane & B2 dichloromethan \\
\hline 3.4 & 62.6 & A1 hexane & B2 dichloromethan \\
\hline & & & \\
\hline & & & \\
\hline
\end{tabular}

Page 1 of 1 


\section{Synthetic Methods}

The final purification was done by 7 reverse phase HPLC (TABLE 7.4) runs, were a solution of the crude oil dissolved in methanol $(15 \mathrm{~mL})$ was used to load the HPLC column. The product was the last major peak observed at $\sim 44$ minute mark. The HPLC product fractions were combined, then concentrated by rotatory evaporator to an orange oil ( $2.47 \mathrm{~g}, 44.5 \%$ yield $)$.

Notes: The toluene was an attempt to azeotropically remove any water in the reaction, however the vapor condensed before reaching the Dean-Stark trap, leaving water droplets on the glassware. The toluene definitely doesn't hurt the reaction but it is unclear if it helps. Also, with a larger batch, it may be possible to distill the product under high vacuum.

TABLE 7.4: HPLC Conditions used to purify G4-068s16

\begin{tabular}{|c|c|c|c|}
\hline & $\begin{array}{c}\text { Supelco } \\
\text { Discovery } 18 \\
(\mathrm{C} 18,25 \mathrm{~cm} \times \\
21.2 \mathrm{~mm}, 5 \mu \mathrm{m} \\
(\text { cat\#:569226- } \\
\text { U) })\end{array}$ & $\begin{array}{c}\text { Gradient } \\
\text { (min.) }\end{array}$ & \% B \\
\hline Flow & $20 \mathrm{~mL} / \mathrm{min}$ & $\mathbf{0}$ & 50 \\
\hline$\lambda_{\mathbf{1}}$ & $254 \mathrm{~nm}$ & $\mathbf{5}$ & 50 \\
\hline$\lambda_{\mathbf{2}}$ & $280 \mathrm{~nm}$ & $\mathbf{5 5}$ & 100 \\
\hline Solvent A: & water & $\mathbf{6 0}$ & 100 \\
\hline Solvent B: & acetonitrile & $\mathbf{-}$ & - \\
\hline
\end{tabular}

${ }^{1} \mathrm{H}-\mathrm{NMR} \delta(\mathrm{ppm})\left(\right.$ DMSO-d $\left._{6}\right): 10.337(\mathrm{~s}, 1 \mathrm{H}), \delta 7.380(\mathrm{dd}, \mathrm{J}=9.0 \mathrm{~Hz}, 3.0 \mathrm{~Hz}$, 1H), $7.189(\mathrm{~d}, \mathrm{~J}=3.0 \mathrm{~Hz}, 1 \mathrm{H}), 7.289(\mathrm{~d}, \mathrm{~J}=9.0 \mathrm{~Hz}, 1 \mathrm{H}), 7.210-7.26(\mathrm{~m}, 2 \mathrm{H}), 7.03-$ $7.08(\mathrm{~m}, 2 \mathrm{H}), 4.132(\mathrm{t}, \mathrm{J}=6.6 \mathrm{~Hz}, 2 \mathrm{H}), 1.75-1.81(\mathrm{~m}, 2 \mathrm{H}), 1.42-1.49(\mathrm{~m}, 2 \mathrm{H})$, $1.28-1.36(\mathrm{~m}, 4 \mathrm{H}), 0.885(\mathrm{t}, \mathrm{J}=6.9 \mathrm{~Hz}, 3 \mathrm{H})$. 


\section{Biological Assays: TEVC Methods}

${ }^{13} \mathrm{C}-\mathrm{NMR} \delta(\mathrm{ppm})\left(\mathrm{DMSO}_{\mathrm{d}}\right)$ ): 188.54, 158.93, 157.36, 152.99, 150.35,

$126.94,124.83,120.16,116.61,115.96,115.77,68.90,30.90,28.43,25.09,22.00$, 13.86 .

${ }^{19}$ F-NMR $\delta(\mathrm{ppm})\left(\right.$ DMSO- $\mathrm{d}_{6}$, with $\mathrm{C}_{6} \mathrm{~F}_{6}$ as reference at $\left.-164.9 \mathrm{ppm}\right):-122.17$ to -122.26 .

TABLE 7.5: High resolution MS (ESI) of 5-(4-fluorophenoxy)-2-(hexyloxy)benzaldehyde (G4-068s20)

\begin{tabular}{|c|c|c|c|}
\hline Species & Chemical Formula & Calculated $\boldsymbol{m} / \mathbf{z}$ & Observed $\boldsymbol{m} / \mathbf{z}$ \\
\hline$[\mathbf{M}+\mathbf{H}]^{+}$ & $\mathbf{C}_{\mathbf{1 9}} \mathbf{H}_{\mathbf{2 2}} \mathbf{F O}_{\mathbf{3}}{ }^{+}$ & 317.15475 & 371.16291 \\
\hline$[\mathbf{M}+\mathbf{N a}]^{+}$ & $\mathbf{C}_{\mathbf{1 9}} \mathbf{H}_{\mathbf{2 1}} \mathbf{F N a O}_{3}{ }^{+}$ & 339.13669 & 339.13728 \\
\hline$[\mathbf{M}+\mathbf{M e O H}+\mathbf{N a}]^{+\mathbf{a}}$ & $\mathbf{C}_{\mathbf{2 0}} \mathbf{H}_{\mathbf{2 5}} \mathbf{F N a O}_{\mathbf{4}}{ }^{+}$ & 371.16291 & 371.16289 \\
\hline
\end{tabular}

a. This is the sodium cation form of the hemiacetal with $\mathrm{MeOH}$.

\subsection{Biological Assays: TEVC Methods}

7.2.1 In Vitro Transcription (used with permission from Dr. Yohei Norimatsu)

Human CFTR cDNA was used in a modified pBluescript vector (Agilent Technologies, Santa Clara, CA) containing T7 promoter and the 5' untranslated region of the Xenopus $\beta$-globin gene upstream from the coding sequence of CFTR.

Human TAAR1 cDNA was used in the pcDNA3.1 vector (Life Technologies, Carlsbad, CA). The DNA sequences were confirmed by direct DNA sequencing. The cRNAs for Xenopus oocyte injection were synthesized using the mMessage mMachine T7-Ultra ${ }^{\mathrm{TM}}$ in vitro transcription kit from Life Technologies (Carlsbad, CA). After transcription, poly(A) tails were added to the transcripts using E. coli 


\section{Biological Assays: TEVC Methods}

Poly(A) polymerase as described in the mMessage mMachine T7 Ultra transcription kit.

7.2.2 Preparation and Microinjection of Oocytes (used with permission from Dr. Yohei Norimatsu)

Female Xenopus Laevis frogs were anesthetized by immersion in cold water containing Tricaine, $3 \mathrm{mg} / \mathrm{ml}$ (Sigma Chemical Co., St. Louis, MO). The oocytes were removed through a small abdominal incision which was then closed by 4.0 nylon suture. The follicular membranes were removed by mechanical agitation (12 h) in a $\mathrm{Ca}^{2+}$-free solution containing $82.5 \mathrm{mM} \mathrm{NaCl}, 2 \mathrm{mM} \mathrm{KCl}, 1 \mathrm{mM} \mathrm{MgCl} 2,5$ mM HEPES (pH 7.5), and 0.2 Wünsch units/mL Liberase Blendzyme ${ }^{\mathrm{TM}}$ (Roche Molecular Biochemicals, Indianapolis, IN). We selected Stage V and VI defolliculated oocytes, which were then washed and incubated at $18^{\circ} \mathrm{C}$ in a modified Barth's solution (MBSH) containing $88 \mathrm{mM} \mathrm{NaCl}, 1 \mathrm{mM} \mathrm{KCl}, 0.82 \mathrm{mM} \mathrm{MgSO}_{4}$, $0.33 \mathrm{mM} \mathrm{Ca}\left(\mathrm{NO}_{3}\right)_{2}, 0.41 \mathrm{mM} \mathrm{CaCl}_{2}, 2.4 \mathrm{mM} \mathrm{NaHCO}_{3}, 10 \mathrm{mM}$ HEPES-Hemi$\mathrm{Na}$, and $250 \mathrm{mg} / \mathrm{L}$ Amikacin with $150 \mathrm{mg} / \mathrm{L}$ Gentamicin (pH 7.5) until injection the next day. Each oocyte was injected with $50 \mathrm{nl}$ of RNA solution containing 100 ng/ $\mu 1 \mathrm{hTAAR} 1$ and $10 \mathrm{ng} / \mu \mathrm{l}$ hCFTR cRNA. Injected oocytes were incubated at $18{ }^{\circ} \mathrm{C}$ in 12-well plates containing MBSH. Injection pipettes were pulled from filamented glass capillary tubes (Sutter Instrument, Novato CA) on a P-97 Flaming Brown micropipette puller (Sutter Instrument, Novato CA). Typically, the oocytes were used 3 to 7 days after injection. 


\section{Biological Assays: TEVC Methods}

\subsubsection{Whole Cell Recordings (used with permission from Dr. Yohei Norimatsu)}

Individual oocytes were placed in a $500 \mu \mathrm{L} \mathrm{RC}-1 \mathrm{Z}$ recording chamber (Warner Instruments, Hamden CT) and continuously perfused with Frog Ringer's solution ( $\sim 5 \mathrm{~mL} / \mathrm{min})$ via a siphon. The Ringer's solution contained $98 \mathrm{mM} \mathrm{NaCl}, 2 \mathrm{mM}$ $\mathrm{KCl}, 1 \mathrm{mM} \mathrm{MgCl} 2,1.8 \mathrm{mM} \mathrm{CaCl}_{2}$, and $5 \mathrm{mM}$ HEPES (hemi-Na) (pH 7.4). Oocytes were initially maintained in the experimental chamber under open circuit conditions and experiments began when the transmembrane voltage was between $25 \mathrm{mV}$ and $-40 \mathrm{mV}$. The membrane potential was ramped from $-120 \mathrm{mV}$ to +60 $\mathrm{mV}$ in $1.8 \mathrm{~s}$.

Membrane currents were recorded from oocytes with a two-electrode voltage clamp using an amplifier (TEV-200; Dagan, Minneapolis, MN) at a room temperature of $\sim 22^{\circ} \mathrm{C}$. Current-injecting and potential-measuring electrodes had resistances of $\sim 1.0-3.0 \mathrm{M} \Omega$, when filled with $3 \mathrm{M} \mathrm{KCl}$. The bath solution was connected to the ground via a low-resistance agarose bridge containing $2 \%$ agarose in $3 \mathrm{M} \mathrm{KCl}$. Current measurements were low-pass filtered at $0.5 \mathrm{kHz}$. Data acquisition was performed on a Pentium-based microcomputer using pCLAMP software and an analog-to-digital converter (Axon Instruments, Foster City, CA).

\subsubsection{Compound Assays}

A $100 \mathrm{mM}$ stock solution of each test compound, in DMSO, was made, and then used to make the test solutions. 


\section{Biological Assays: TEVC Methods}

With the exception of the first and last compound assay (FIGURE 7.4 and

7.6(1), respectfully), all assays followed a head-to-head competition assay paradigm where a 1.5 minute pulse of $10 \mu \mathrm{M}$ Meth in Frog Ringer's solution (FR) was perfused over an oocyte, injected with hTAAR1 and hCFTR encoding RNA, followed by a wash-out period with FR. After the initial Meth pulse and wash-out, a series of 1.5 minute pulse of $10 \mu \mathrm{M}$ Meth and test compound ( $1 \mathrm{nM}$ to $10 \mu \mathrm{M})$ in FR followed by FR wash-out cycles were preformed. At the end of each experiment, a 1.5 minute pulse of $10 \mu \mathrm{M}$ forskolin and $10 \mu \mathrm{M}$ of the test compound in FR followed by a FR wash-out period. The wash-out periods lasted between 15 and 25 minutes (long enough for the conductance to stabilize).

The assay shown in FIGURE 4.4 used an oocyte injected with hTAAR1 and hCFTR encoding RNA. The Meth pulses were 1.5 minutes long and at $100 \mu \mathrm{M}$ concentration in FR. Each Meth pulse was followed by a 15 minute FR wash-out period. After the second pulse of Meth, $20 \mu \mathrm{M}$ G5-109s8 in FR was applied immediately followed by a Meth pulse. No forskolin control was done in this experiment.

The assay shown in FIGURE 4.6(1) used an oocyte injected with hCFTR encoding RNA. Between each pulse of testing solution, the oocyte was washed with FR. The 1.5 minute pulses used were: $5 \mu \mathrm{M}$ forskolin, $10 \mu \mathrm{M}$ G5-109s8, 10 


\section{Biological Assays: TEVC Methods}

$\mu \mathrm{M}$ Meth, and a combination of $5 \mu \mathrm{M}$ forskolin and $10 \mu \mathrm{M}$ G5-109s8. All of these

solutions were made using FR. 


\section{References \& Notes}

1. Katzung, B.G. Basic and Clinical Pharmacology, 11th ed.; Lange Medical Books/McGraw Hill: New York, 2007; pp 557 and 565.

2. Ibid., p 556.

3. Wikipedia: Dopamine (accessed Feb. 6, 2013), http://en.wikipedia.org/wiki/ Dopamine

4. Bunzow, J. R.; et al.; Amphetamine, 3,4-methylenedioxymethamphetamine, lysergic acid diethylamide, and metabolites of the catecholamine neurotransmitters are agonists of a rat trace amine receptor. Mol. Pharmacol. 2001, 60(6), 1181-1188.

5. Achat-Mendes, C., et al., Augmentation of methamphetamine-induced behaviors in transgenic mice lacking the trace amine-associated receptor 1. Pharmacol. Biochem. Behav. 2012. 101(2), 201-207.

6. Miller, G. M. The emerging role of trace amine-associated receptor 1 in the functional regulation of monoamine transporters and dopaminergic activity. J. Neurochem. 2011, 116(2), 164-176.

7. Borowsky, B.; et al.; Trace amines: identification of a family of mammalian G protein-coupled receptors. Proc. Natl. Acad. Sci. U.S.A. 2001. 98(16), 8966-8971.

8. Revel, F. G.; et al.; A new perspective for schizophrenia: TAAR1 agonists reveal antipsychotic- and antidepressant-like activity, improve cognition and control body weight. Mol. Psychiatry, DOI: 10.1038/mp.2012.57, Published online May 30, 2012, http://www.ncbi.nlm.nih.gov/pubmed/22641180.

9. Revel, F. G.; et al.; Trace amine-associated receptor 1 partial agonism reveals novel paradigm for neuropsychiatric therapeutics. Biol. Psychiatry, 2012, 72(11), 934-942.

10. Miller, G. M. Avenues for the development of therapeutics that target trace amine associated receptor 1 (TAAR1). J. Med. Chem. 2012, 55(5), 18091814.

11. Bradaia, A.; et al.; The selective antagonist EPPTB reveals TAAR1-mediated regulatory mechanisms in dopaminergic neurons of the mesolimbic system. Proc. Natl. Acad. Sci. U.S.A, 2009, 106(47), 20081-20086.

12. Smith, S. B.; et al.; Large candidate gene association study reveals genetic risk factors and therapeutic targets for fibromyalgia. Arthritis Rheum. 2012, 64(2), 584-593. 
13. Wasik, A. M.; et al.; Evidence for functional trace amine associated receptor1 in normal and malignant B cells. Leuk. Res. 2012, 36(2), 245-249.

14. Salahpour, A.; et al.; BRET biosensors to study GPCR biology, pharmacology, and signal transduction. Front. Endocrinol. (Lausanne), 2012, 3, 105 and references therein.

15. A survey of the literature revealed a paucity of information on what happens to the GDP after it is released from the TAAR $1-\mathrm{G}_{\alpha \mathrm{s}} / \mathrm{G}_{\beta / \gamma}$ complex.

16. Wettschureck, N.; Offermanns, S. Mammalian G proteins and their cell type specific functions. Physiol. Rev. 2005, 85(4), 1159-1204.

17. Tan, E.S. Understanding the molecular basis of trace amine-associated receptor 1 activation by thyronamines and related analogs. Ph.D. Dissertation, University of California, San Francisco, CA, 2007.

18. (a) Piehl, S.: et al.; Thyronamines-past, present, and future. Endocr. Rev. 2011, 32(1), 64-80 and references therein. (b) Scanlan, T. S.; et al.; 3-Iodothyronamine is an endogenous and rapid-acting derivative of thyroid hormone. Nat. Med. 2004, 10, 638 - 642

19. Panas, H. N.; et al.; Normal thermoregulatory responses to 3-iodothyronamine, trace amines and amphetamine-like psychostimulants in trace amine associated receptor 1 knockout mice. J. Neurosci. Res. 2010, 88(9), 1962-1969.

20. Simmler, L.; et al.; Pharmacological characterization of designer cathinones in vitro. Br. J. Pharmacol. 2013, 168(2), 458-470.

21. Katzung, B. G. Basic and Clinical Pharmacology, 11th ed.; Lange Medical Books/McGraw Hill: New York, 2007; pp 134, 141, 144-145, and 553-568.

22. Benjamin, J.; et al.; Population and familial association between the D4 dopamine receptor gene and measures of Novelty Seeking. Nat. Genet. 1996, 12(1), 81-84.

23. Ebstein, R. P.; et al.; Dopamine D4 receptor (D4DR) exon III polymorphism associated with the human personality trait of Novelty Seeking. Nat. Genet. 1996, 12(1), 78-80.

24. Ikemoto, S.; Panksepp, J. The role of nucleus accumbens dopamine in motivated behavior: a unifying interpretation with special reference to rewardseeking. Brain Res. Rev. 1999, 31(1), 6-41.

25. Pessiglione, M.; et al.; Dopamine-dependent prediction errors underpin reward-seeking behaviour in humans. Nature, 2006, 442(7106), 1042-1045.

26. Roitman, M. F.; et al.; Dopamine operates as a subsecond modulator of food seeking. J. Neurosci. 2004, 24(6), 1265-1271.

27. Suhara, T.; et al.; Dopamine D2 receptors in the insular cortex and the personality trait of novelty seeking. Neuroimage, 2001, 13(5), 891-895. 
28. Wikipedia: Methylphenidate (accessed May 5, 2013), http://en.wikipedia.org/wiki/Methylphenidate\#ADHD_and_stimulant_dynamics_in_general.

29. Wikipedia: Anxiety (accessed May 5, 2013), http://en.wikipedia.org/wiki/ Anxiety.

30. Wikipedia: Norepinephrine (accessed May 6, 2013), http://en.wikipedia.org/ wiki/Norepinephrine

31. Wikipedia: Atomoxetine (accessed May 7, 2013), http://en.wikipedia.org/ wiki/Atomoxetine.

32. Wikipedia: Serotonin (accessed May 6, 2013), http://en.wikipedia.org/wiki/ Serotonin

33. Wikipedia: Serotonin Syndrome (accessed May 6, 2013), http://en.wikipedia.org/wiki/Serotonin_syndrome

34. Galley, G.; et al.; Preparation of substituted benzamide derivatives for use as CNS agents, WO 2009016088 A1, 2009.

35. Wikipedia: Cystic fibrosis tranmembrane conductance regulatory (accessed May 15, 2013), http://en.wikipedia.org/wiki/Cystic fibrosis transmembrane_conductance_regulator.

36. Tallman, K.; et al.; Pharmacologic and Genetic Manipulation of Trace Amine-Associated Receptor 1 Signaling In Mice Demonstrates its Role in Methamphetamine-Stimulated Activity, to be submitted for publication, 2012.

37. Galley, G.; et al.; Optimisation of imidazole compounds as selective TAAR1 agonists: discovery of RO5073012. Bioorg Med Chem Lett, 2012, 22(16), 5244-5248.

38. Galley, G.; Norcross, R.; Polara, A.; Preparation of aminooxazoline derivatives for use as TAAR1 ligands. US 20110112080 A1, 2011.

39. Galley, G.; et al.; 4,5-Dihydrooxazol-2-yl derivatives as TAAR gene expression agents and their preparation, pharmaceutical compositions and use in the treatment of diseases. US 20100029589 A1, 2010.

40. Galley, G.; et al.; Preparation of 4,5-dihydro-oxazol-2-yl derivatives with selectivity for trace amine associated receptors (TAAR) for treatment of diseases, WO 2010010014 A1, 2010.

41. Decoret, G.; et al.; Preparation of 2-aminooxazolines as trace amine associated receptor 1 (TAAR1) ligands, WO 2010139707 A1, 2010.

42. Galley, G.; et al.; Preparation of nicotinamide derivatives as TAARl ligands for the treatment of CNS disorders, US 20090036452 A1, 2009.

43. Galley, G.; et al.; Preparation of 2-imidazolines as trace amine associated receptors modulators, WO 2009003868 A2, 2009.

44. Galley, G.; et al.; Preparation of 2-imidazolines as antidepressants, WO 2009003867 A1, 2009. 
45. Galley, G.; et al.; Preparation of 2-azetidinemethaneamines and 2-pyrrolidinemethaneamines as TAAR ligands, US 20090029962 A1, 2009.

46. Galley, G.; et al.; Imidazole derivatives as TAAR modulators and their preparation and pharmaceutical compositions, US 20080146523 A1, 2008.

47. Galley, G.; et al.; Preparation of aminooxazoline derivatives for use as TAAR1 ligands, WO 2008098857 A1, 2008.

48. Galley, G.; et al.; Preparation of aminooxazoline derivatives for use as central nervous system agents, WO 2008092785 A1, 2008.

49. Galley, G.; et al.; Preparation of novel substituted 2-imidazoles as ligands for trace amine associated receptors (TAAR), WO 2008071574 A1, 2008.

50. Galley, G.; et al.; Preparation of substituted 4-imidazoles having good affinity to the trace amine associated receptors (TAARs), WO 2008058867 A2, 2008.

51. Galley, G.; et al.; Preparation of substituted 2-imidazoles as modulators of the trace amine associated receptors, WO 2008052907 A1, 2008.

52. Galley, G.; et al.; Aminomethyl-2-imidazoles with affinity with the trace amine associated receptors and their preparation, pharmaceutical compositions and use in the treatment of diseases, WO 2008046756 A1, 2008.

53. Galley, G.; et al.; Aminomethyl-4-imidazoles and their preparation, pharmaceutical compositions and use in the treatment of diseases, WO 2008046757 A1, 2008.

54. Galley, G.; et al.; Preparation of 2-imidazoles for the treatment of CNS disorders, WO 2007085558 A1, 2007.

55. Galley, G.; et al.; Preparation of aralkylimidazoles as ligands of trace amine associated receptors, especially TAAR1, WO 2007085556 A2, 2007.

56. Galley, G.; et al.; Preparation of substituted 2-imidazole imidazoline derivatives for the treatment of diseases related to trace amine associated receptors, WO 2007085557 A2, 2007.

57. Scanlan, T.S.; et al.; Thyronamine derivatives and analogs and methods of use thereof, US 20050096485 A1, 2005.

58. Naganathan, S., Exelixis Inc.,South San Francisco, CA, Personal communication, c. 2002.

59. Imanishi, M.; et al.; Discovery of a Novel Series of Benzoic Acid Derivatives as Potent and Selective Human $\beta_{3}$ Adrenergic Receptor Agonists with Good Oral Bioavailability. Phenylethanolaminotetraline (PEAT) Skeleton Containing Biphenyl or Biphenyl Ether Moiety. J. Med. Chem. 2008, 51, 4804-4822. 
60. Tipparaju, S. K.; et al.; Design and synthesis of aryl ether inhibitors of the Bacillus anthracis enoyl-ACP reductase. ChemMedChem, 2008, 3, 12501268.

61. Zhu, L.; Duquette, J.; Zhang, M.; An Improved Preparation of Arylboronates: Application in One-Pot Suzuki Biaryl Synthesis. J. Org. Chem. 2003, 68, 3729-3732.

62. Chen, Z.; et al.; A New Multidentate Hexacarboxylic Acid for the Construction of Porous Metal-Organic Frameworks of Diverse Structures and Porosities. Cryst. Growth Des. 2010, 10, 2775-2779.

63. Esumi, T.; et al.; Efficient synthesis and structure-activity relationship of honokiol, a neurotrophic biphenyl-type neolignan. Bioorg. Med. Chem. Lett. 2004, 14, 2621-2625.

64. Inglis, S. R.; et al.; Synthesis and Evaluation of 3-(Dihydroxyboryl)benzoic Acids as D,D-Carboxypeptidase R39 Inhibitors. J. Med. Chem. 2009, 52, 6097-6106.

65. Ishiyama, T.; Murata, M.; Miyaura, N. Palladium(0)-Catalyzed Cross-Coupling Reaction of Alkoxydiboron with Haloarenes: A Direct Procedure for Arylboronic Esters. J. Org. Chem. 1995, 60, 7508-10.

66. Jin, S.; et al.; Synthesis, evaluation, and computational studies of naphthalimide-based long-wavelength fluorescent boronic acid reporters. Chem.Eur. J. 2008, 14, 2795-2804.

67. McKiernan, G. J.; Hartley, R. C. Boronate titanium alkylidene reagents for diversity-based synthesis of benzofurans. Org. Lett. 2003, 5(23), 4389-92.

68. DiMauro, E. F.; Vitullo, J. R. Microwave-Assisted Preparation of Fused Bicyclic Heteroaryl Boronates: Application in One-Pot Suzuki Couplings. J. Org. Chem. 2006, 71, 3959-3962.

69. Ley, S.V.; Thomas, A. W. Modern synthetic methods for copper-mediated C(aryl)-O, C(aryl)-N, and C(aryl)-S bond formation. Angew. Chem., Int. Ed. 2003, 42, 5400-5449.

70. Kürti, L.S.; Czakó, B. Strategic applications of named reactions in organic synthesis: background and detailed mechanisms; Elsevier Academic Press 1: Amsterdam; Boston, 2005; pp 464-467.

71. Sigma-Aldrich: Falling-film distillation heads (accessed June 1, 2012), http:/ /www.sigmaaldrich.com/catalog/search?interface=All\&term=fallingfilm $\% 20 \&$ lang $=$ en\&region $=\mathrm{US} \&$ focus $=$ product $\& \mathrm{~N}=$ $0+220003048+219853269+219853286 \mathrm{p}$.

72. Van Allen, D. Methodology and Mechanism: Reinvestigating the Ullmann Reaction, Ph.D. Dissertation, University of Massachusetts, Amherst, MA, 2004 
73. Fan, X. Y.; et al.; $\mathrm{AlCl}_{3}$ and BDMAEE: A Pair of Potent Reactive Regulators of Aryl Grignard Reagents and Highly Catalytic Asymmetric Arylation of Aldehydes. Chem.-Eur. J. 2010, 16, 7988-7991.

74. Chen, G.; et al.; Facile Preparation of $\alpha$-Aryl Nitriles by Direct Cyanation of Alcohols with TMSCN Under the Catalysis of $\operatorname{InX} X_{3}$. Org. Lett. 2008, 10, 4573-4576.

75. Beignet, J. LinkedIn.com: Can some one suggest ways to reduce an 2-(2alkoxyphenyl)-2-phenylacetonitrile to a 2-(2-alkoxyphenyl)-2-phenylethanamine?, 2012, http://www.linkedin.com/groups/Can-some-one-suggest-ways923867.S.111649468?qid=c9e503d9-2127-4a41-87bb-31e2304da5c6\&trk=group_items_see_more-0-b-ttl p.

76. Rannug, U.; et al.; International Commission for Protection against Environmental Mutagens and Carcinogens. An evaluation of the genetic toxicity of paracetamol. Mutat. Res. 1995, 327(1-2), 179-200.

77. McGregor, D. Hydroquinone: an evaluation of the human risks from its carcinogenic and mutagenic properties. Crit. Rev. Toxicol. 2007, 37(10), 887914.

78. Cha, J. S.; Kwon, S. S. Exceptionally facile reduction of carboxylic esters to aldehydes by lithium aluminum hydride in the presence of diethylamine. J. Org. Chem. 1987, 52, 5486-5487.

79. Wikipedia: Forskolin (accessed May 15, 2013), http://en.wikipedia.org/wiki/ Forskolin

80. Connors, K.A. Chemical Kinetics: The Study of Reaction Rates in Solution; John Wiley \& Sons: New York, 1990; p 179.

81. ALA Scientific: OctaFlow II Multi-function Multi-valve Perfusion System (accessed May 10, 2013), http://www.alascience.com/products/ index.php?main_page=product_info\&cPath $=1 \_6 \&$ products_id $=57$

82. Suchland, K. Oregon Health \& Science University, Portland, OR, Unpublished work, 2007.

83. By reason of similarity: a logical argument stating that if $\mathbf{A}$ is similar to $\mathbf{B}$, then A will have similar properties to B. In Euclidean geometry, two triangles are similar if their corresponding interior angles are equivalent. By similarity, the length of the sides of one triangle will be proportional to the length of the corresponding sides of the other triangle.

84. Compounds can be designated as agonists, neutral antagonists (does not block basal activity) or inverse agonists (an antagonist that blocks basal activity) when dealing with enzymes and receptors that have basal activity. Depending on how strongly they effect their target proteins, compounds may get the modifier of either "full" or "partial" and sometimes "super." The author dislikes the conventional names, as they can easily lead to confusion, 
and prefers "activator" instead of "agonist" and "inhibitor" instead of "antagonist," with inhibitors being further broken down into "access inhibitors" which prevent other ligands from binding to the protein, "active-site inhibitors" an inhibitor that binds to the active site, "conformational inhibitors" taking the place of "inverse agonist" and which prevent the protein from spontaneously changing into the active form, finally "allosteric inhibitors" which prevent allosteric activation.

85. Connors, K. A. Chemical Kinetics: The Study of Reaction Rates in Solution; John Wiley \& Sons: New York, 1990.

86. Revel, F. G.; et al.; Brain-specific overexpression of trace amine-associated receptor 1 alters monoaminergic neurotransmission and decreases sensitivity to amphetamine. Neuropsychopharmacology. 2012, 37(12), 2580-2592. 


\section{A.1 5-bromo-2-(hexyloxy)benzaldehyde: G4-012s16}

FIGURE A.1: Structure of G4-012s16.
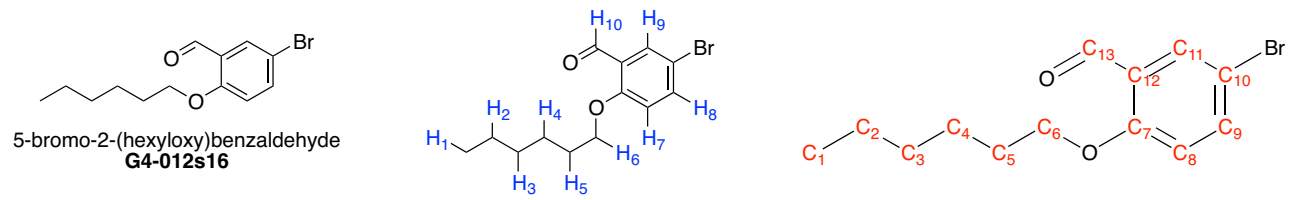

TABLE A.1: ${ }^{1} \mathrm{H}-\mathrm{NMR}$ assignments for G4-012s16, $600 \mathrm{MHz}$ in DMSO (2.50 ppm)

\begin{tabular}{|c|c|c|c|c|c|}
\hline Proton & $\mathbf{p p m}^{\mathrm{a}}$ & Number & Type & $\mathbf{J}_{\mathbf{a}}$ & $\mathbf{J}_{\mathbf{b}}$ \\
\hline $\mathbf{H}_{1}$ & 0.870 & 3 & $\mathbf{t}$ & 6.9 & \\
\hline $\mathrm{H}_{2} \& \mathrm{H}_{3}$ & 1.309 & 4 & m & & \\
\hline $\mathbf{H}_{4}$ & 1.435 & 2 & m & & \\
\hline $\mathbf{H}_{5}$ & 1.763 & 2 & m & & \\
\hline $\mathbf{H}_{6}$ & 4.126 & 2 & $\mathbf{t}$ & 6.6 & \\
\hline $\mathbf{H}_{7}$ & 7.225 & 1 & d & 9.0 & \\
\hline $\mathbf{H}_{8}$ & 7.79 & 1 & dd & 9.0 & 3.0 \\
\hline $\mathbf{H}_{9}$ & 7.724 & 1 & d & 3.0 & \\
\hline $\mathbf{H}_{10}$ & 10.286 & 1 & $\mathbf{S}$ & & \\
\hline
\end{tabular}

a. Taken at the center of the peak.
TABLE A.2: ${ }^{13} \mathrm{C}-\mathrm{NMR}$ assignments for G4-012s16, $600 \mathrm{MHz}$ in DMSO (39.50 ppm)

\begin{tabular}{|c|c|}
\hline Carbon & $p p m$ \\
\hline$C_{1}$ & 13.84 \\
\hline$C_{2}$ & 21.98 \\
\hline$C_{3}$ & 30.87 \\
\hline$C_{4}$ & 25.02 \\
\hline$C_{5}$ & 28.28 \\
\hline$C_{6}$ & 68.82 \\
\hline$C_{7}$ & 160.08 \\
\hline$C_{8}$ & 116.33 \\
\hline$C_{9}$ & 138.45 \\
\hline$C_{10}$ & 112.23 \\
\hline$C_{11}$ & 129.69 \\
\hline$C_{12}$ & 125.7 \\
\hline$C_{13}$ & 187.99 \\
\hline
\end{tabular}


5-bromo-2-(hexyloxy)benzaldehyde: G4-012s16

FIGURE A.2: ${ }^{1}$ H-NMR of G4-012s16.
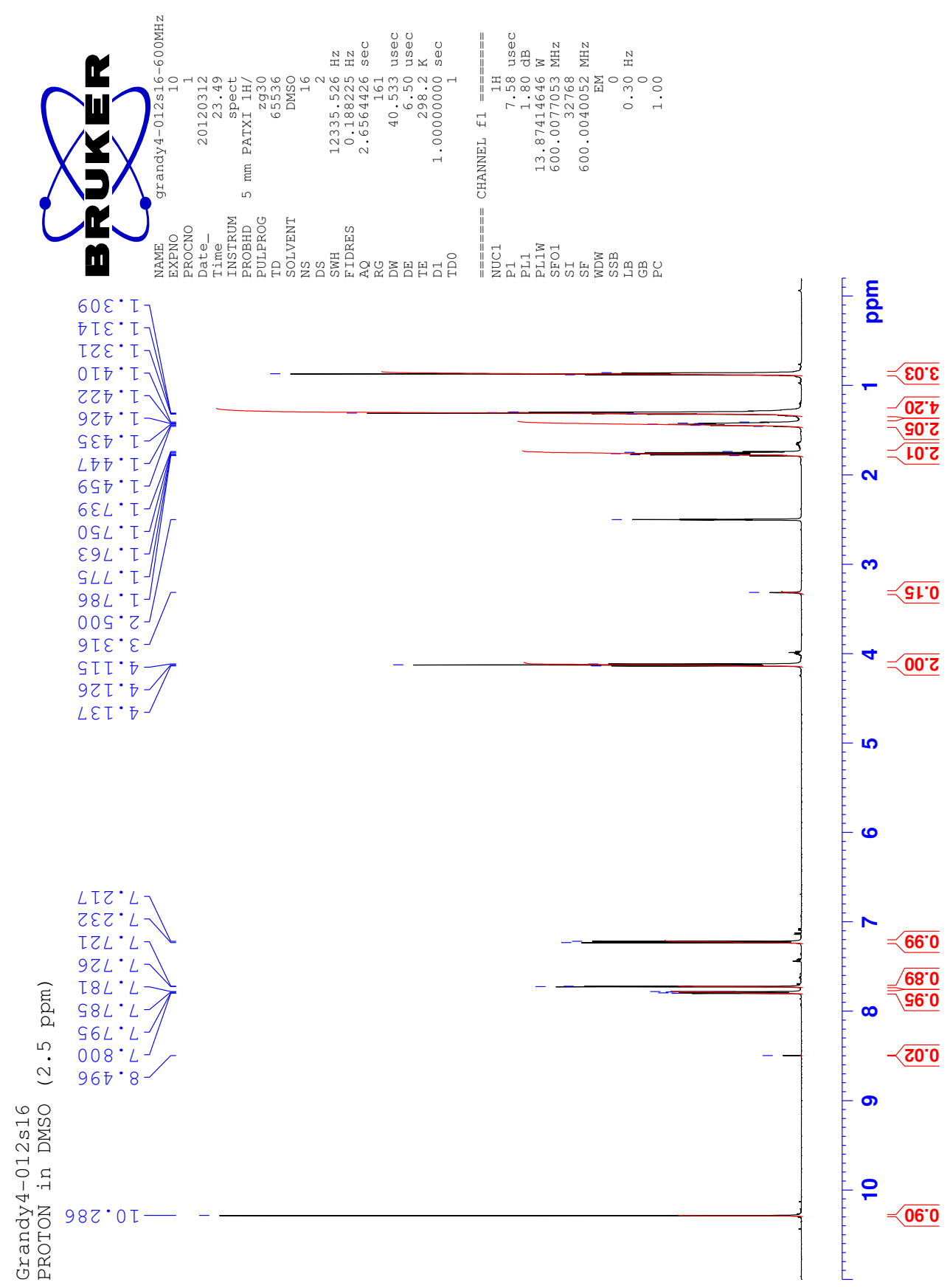
5-bromo-2-(hexyloxy)benzaldehyde: G4-012s16

FIGURE A.3: COSY-NMR of G4-012s16.

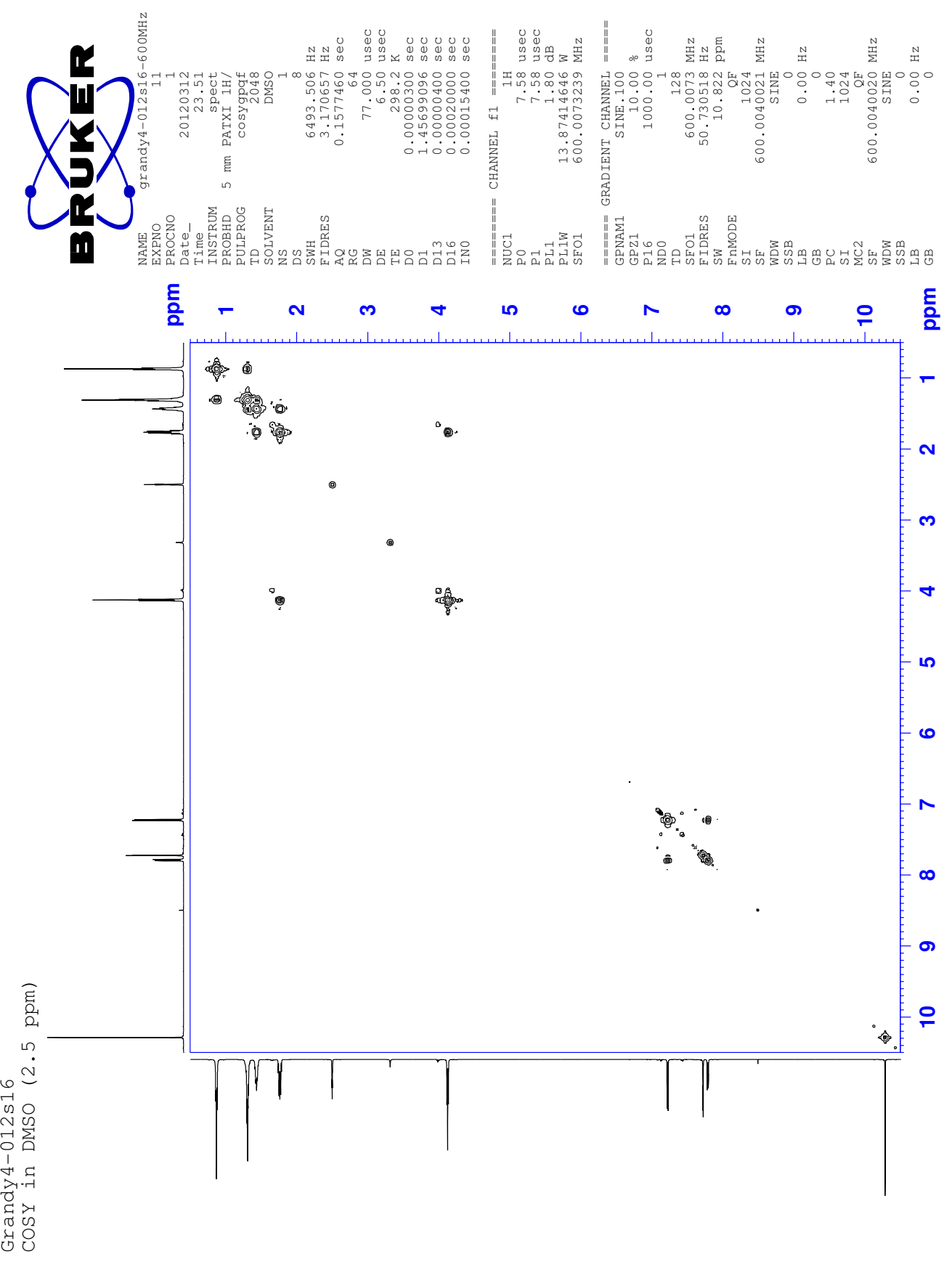


5-bromo-2-(hexyloxy)benzaldehyde: G4-012s16

FIGURE A.4: ${ }^{13} \mathrm{C}-\mathrm{NMR}$ of G4-012s16.

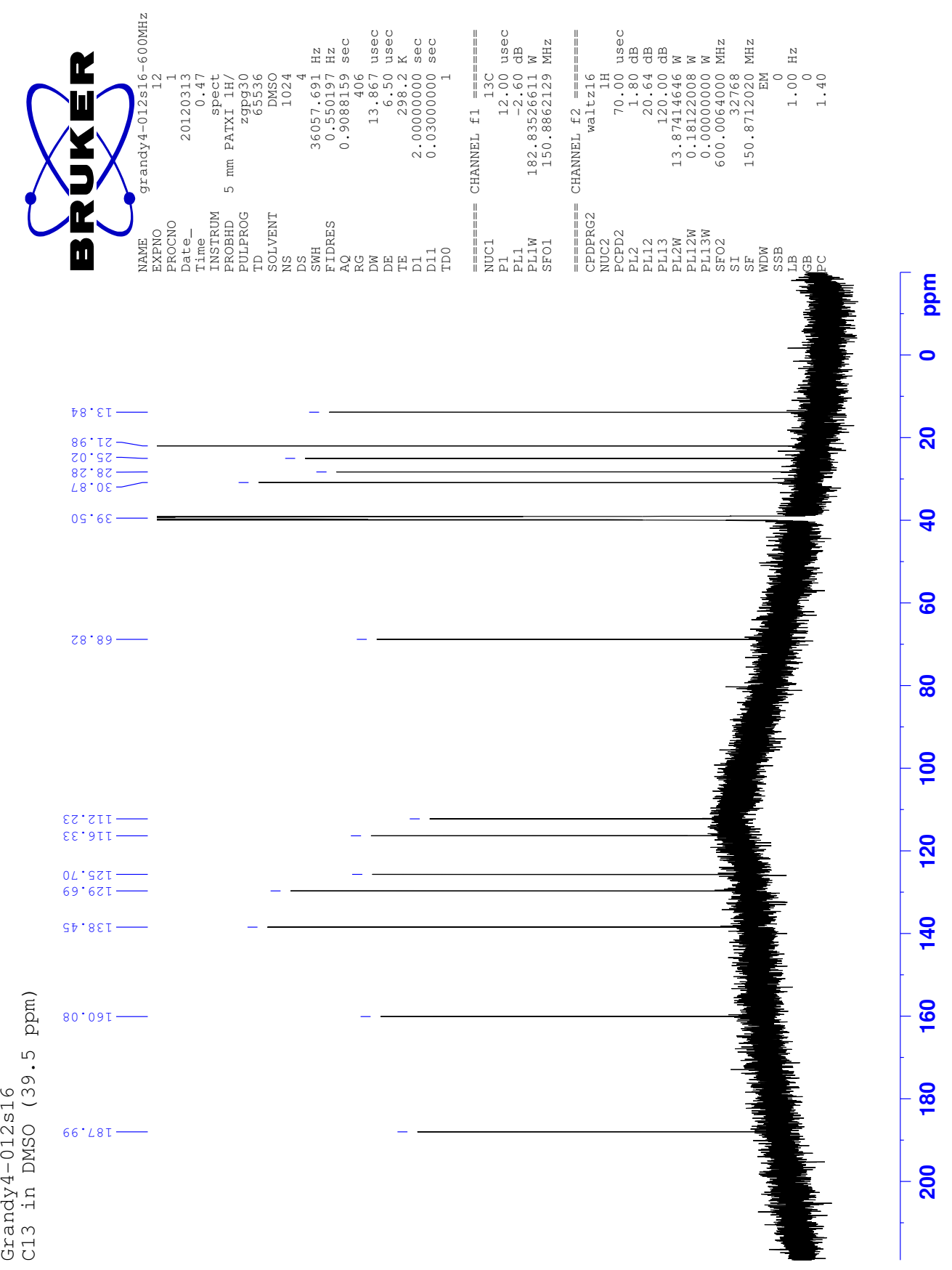


5-bromo-2-(hexyloxy)benzaldehyde: G4-012s16

FIGURE A.5: HSQC-NMR of G4-012s16.

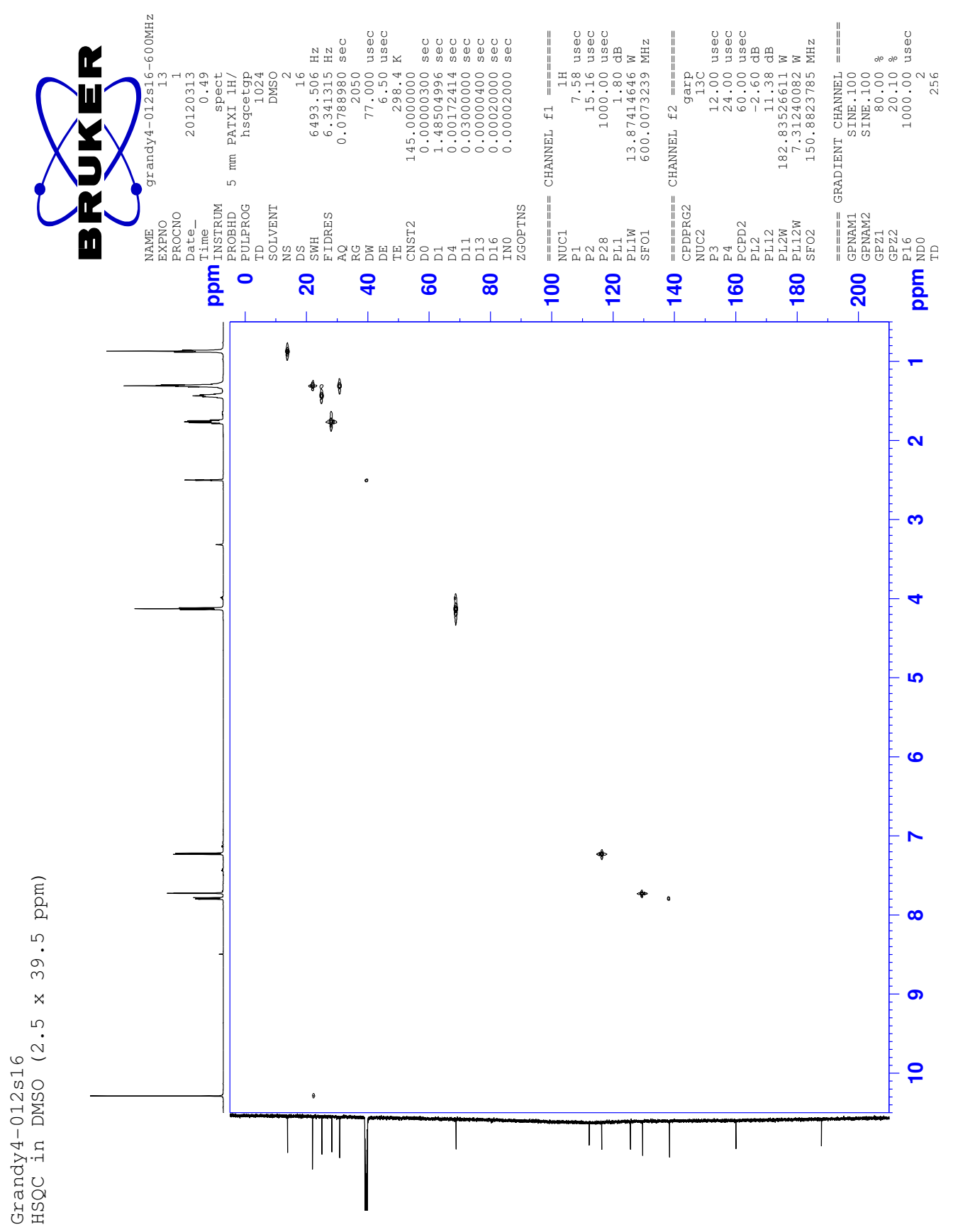


5-bromo-2-(hexyloxy)benzaldehyde: G4-012s16

FIGURE A.6: HMBC-NMR of G4-012s16.

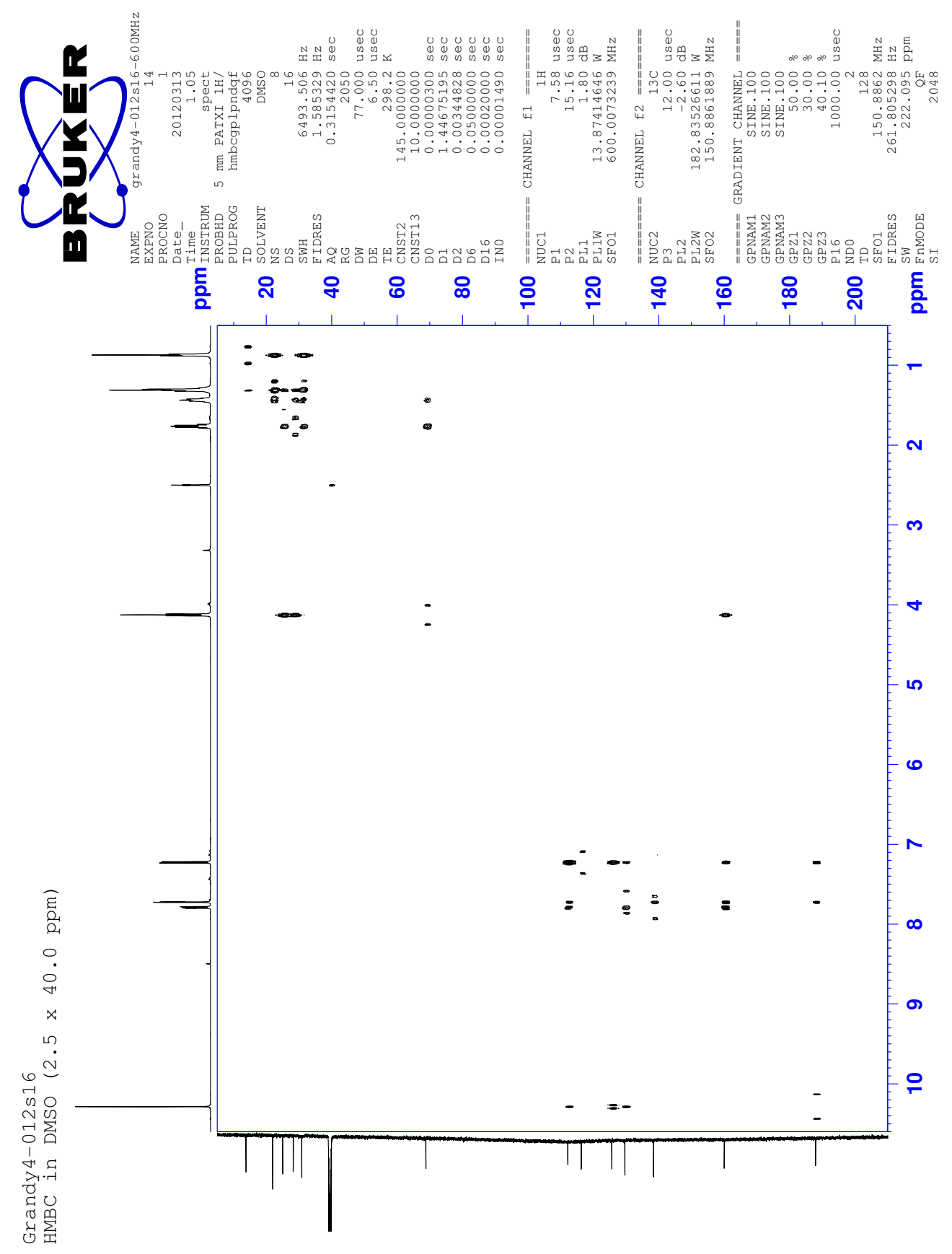


5-bromo-2-(hexyloxy)benzaldehyde: G4-012s16

FIGURE A.7: NOESY-NMR of G4-012s16.

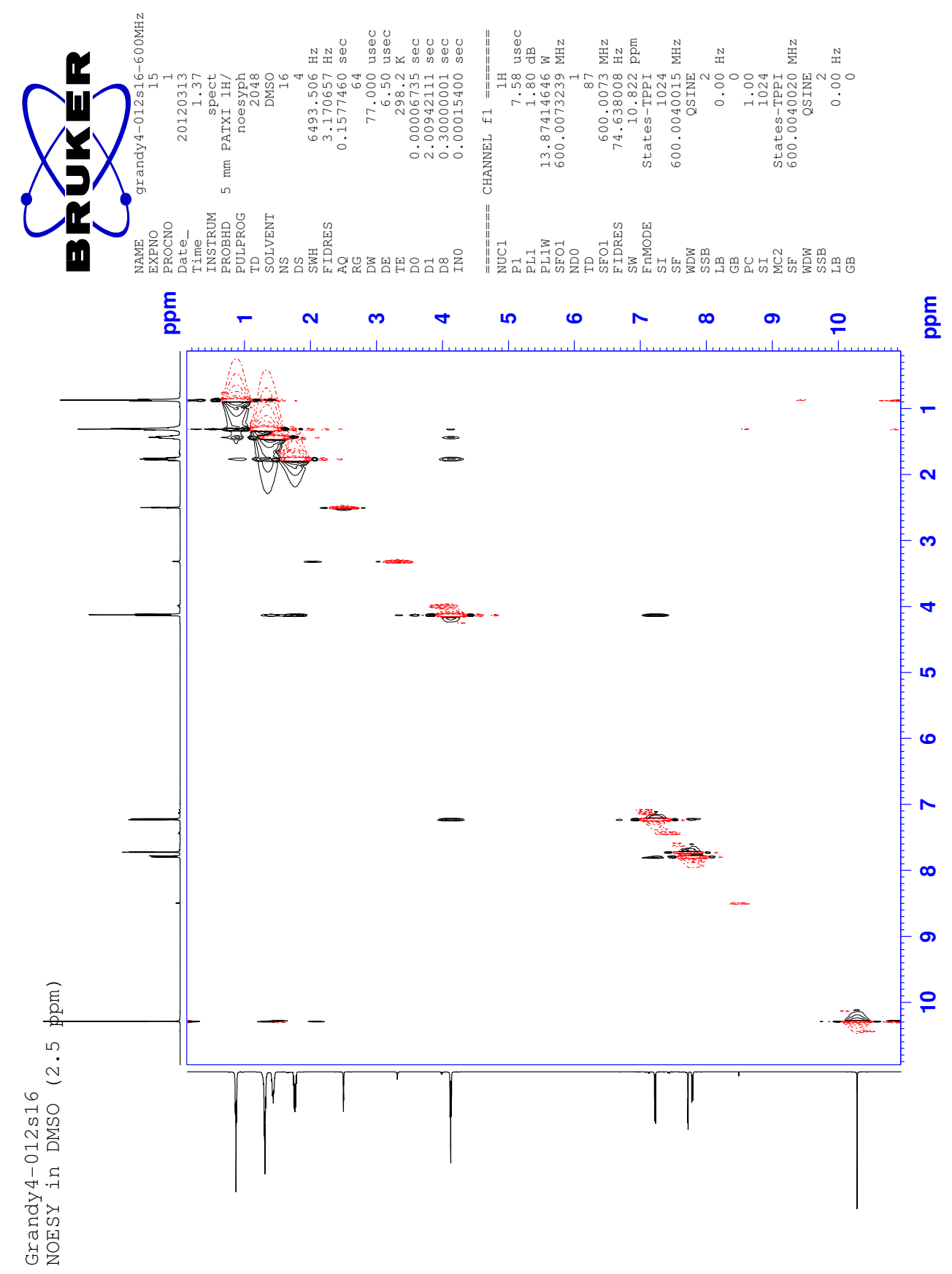


5-(4-fluorophenoxy)-2-(hexyloxy)benzaldehyde: G4-068s20 \& G4-070s6

\section{A.2 5-(4-fluorophenoxy)-2-(hexyloxy)benzaldehyde: G4-068s20 \& G4-070s6}

FIGURE A.8: Structure of G4-070s6.
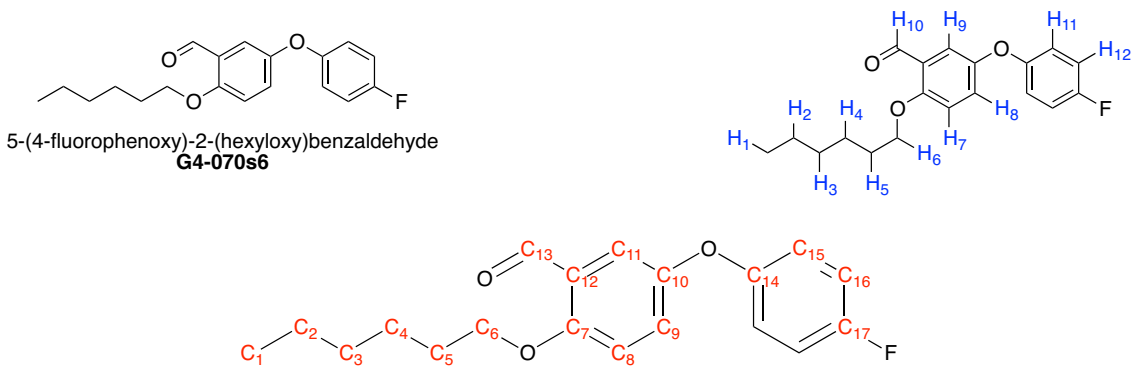

TABLE A.3: ${ }^{1} \mathrm{H}-\mathrm{NMR}$ assignments for G4-070s6, $600 \mathrm{MHz}$ in DMSO (2.50 ppm)

\begin{tabular}{|c|c|c|c|c|c|}
\hline Proton & ppm $^{\mathbf{a}}$ & Number & Type & $\mathbf{J}_{\mathbf{a}}$ & $\mathbf{J}_{\mathbf{b}}$ \\
\hline $\mathrm{H}_{1}$ & 0.885 & 3 & $\mathbf{t}$ & 6.9 & \\
\hline $\mathbf{H}_{2} \& \mathbf{H}_{3}$ & 1.328 & 4 & $\mathbf{m}$ & & \\
\hline $\mathbf{H}_{4}$ & 1.456 & 2 & $\mathbf{m}$ & & \\
\hline $\mathbf{H}_{5}$ & 1.779 & 2 & $\mathbf{m}$ & & \\
\hline $\mathrm{H}_{6}$ & 4.132 & 2 & $\mathbf{t}$ & 6.6 & \\
\hline $\mathbf{H}_{7}$ & 7.289 & 1 & d & 9.0 & \\
\hline $\mathrm{H}_{8}$ & 7.380 & 1 & dd & 9.0 & 3.0 \\
\hline $\mathrm{H}_{9}$ & 7.189 & 1 & d & 3.0 & \\
\hline $\mathrm{H}_{10}$ & 10.337 & 1 & $\mathbf{s}$ & & \\
\hline $\mathbf{H}_{11}$ & 7.231 & 2 & $\mathbf{m}$ & & \\
\hline $\mathrm{H}_{12}$ & 7.054 & 2 & m & & \\
\hline
\end{tabular}

a. Taken at the center of the peak.
TABLE A.4: ${ }^{13} \mathrm{C}-\mathrm{NMR}$ assignments for G4-070s6, $600 \mathrm{MHz}$ in DMSO (39.50 ppm)

\begin{tabular}{|l|l|}
\hline Carbon & ppm \\
\hline$C_{1}$ & 13.86 \\
\hline$C_{2}$ & 22.00 \\
\hline$C_{3}$ & 30.90 \\
\hline$C_{4}$ & 25.09 \\
\hline$C_{5}$ & 28.43 \\
\hline$C_{6}$ & 68.90 \\
\hline$C_{7}$ & 157.36 \\
\hline$C_{8}$ & 115.77 \\
\hline$C_{9}$ & 126.94 \\
\hline$C_{10}$ & 150.35 \\
\hline$C_{11}$ & 115.96 \\
\hline$C_{12}$ & 124.83 \\
\hline$C_{13}$ & 188.54 \\
\hline$C_{14}$ & 152.99 \\
\hline$C_{15}$ & 116.61 \\
\hline$C_{16}$ & 120.16 \\
\hline$C_{17}$ & 158.93 \\
\hline
\end{tabular}


5-(4-fluorophenoxy)-2-(hexyloxy)benzaldehyde: G4-068s20 \& G4-070s6

FIGURE A.9: ${ }^{1} \mathrm{H}-\mathrm{NMR}$ of G4-070s6.

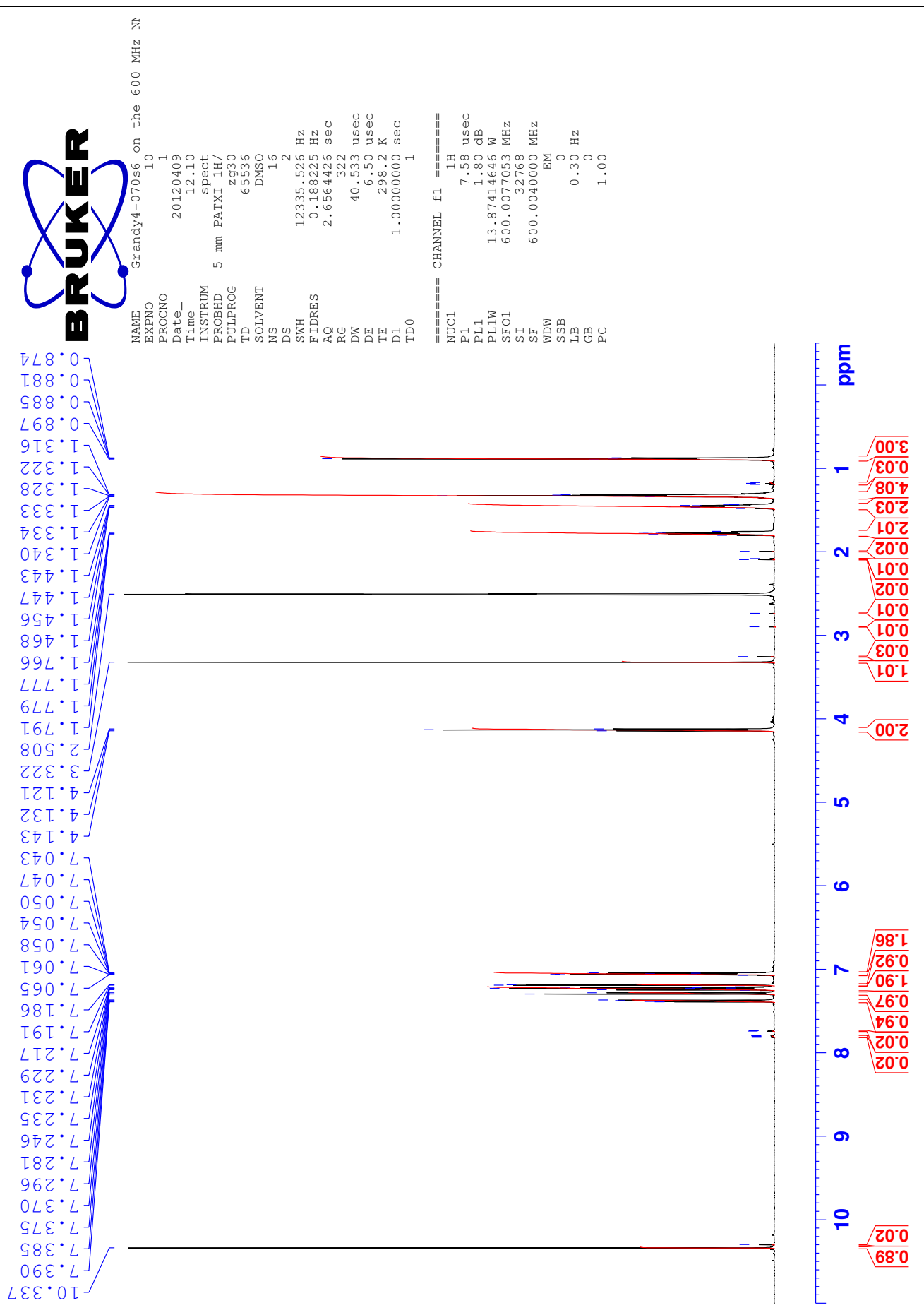


5-(4-fluorophenoxy)-2-(hexyloxy)benzaldehyde: G4-068s20 \& G4-070s6

FIGURE A.10: COSY-NMR of G4-070s6.

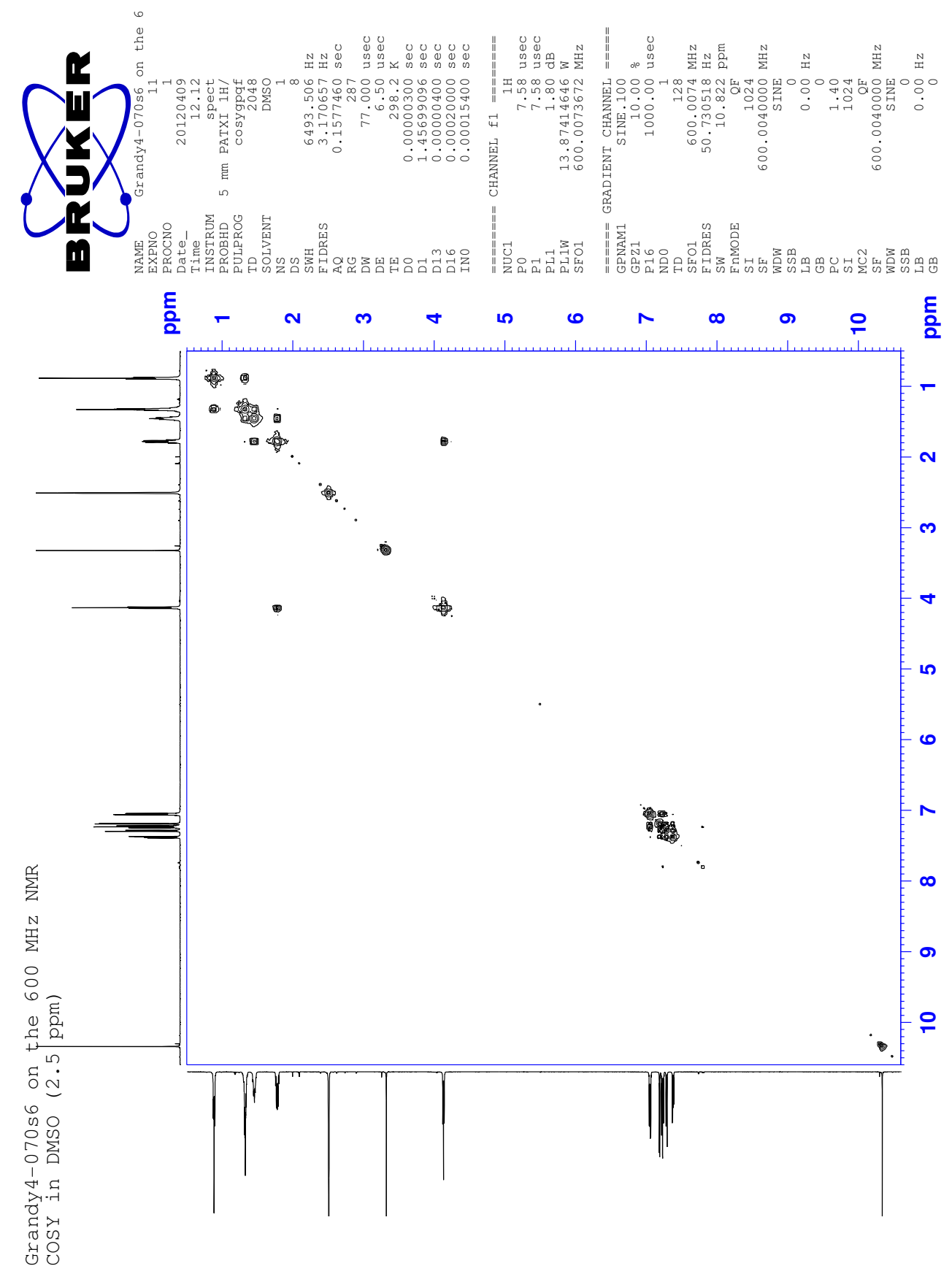


5-(4-fluorophenoxy)-2-(hexyloxy)benzaldehyde: G4-068s20 \& G4-070s6

FIGURE A.11: ${ }^{13} \mathrm{C}-\mathrm{NMR}$ of G4-070s6.

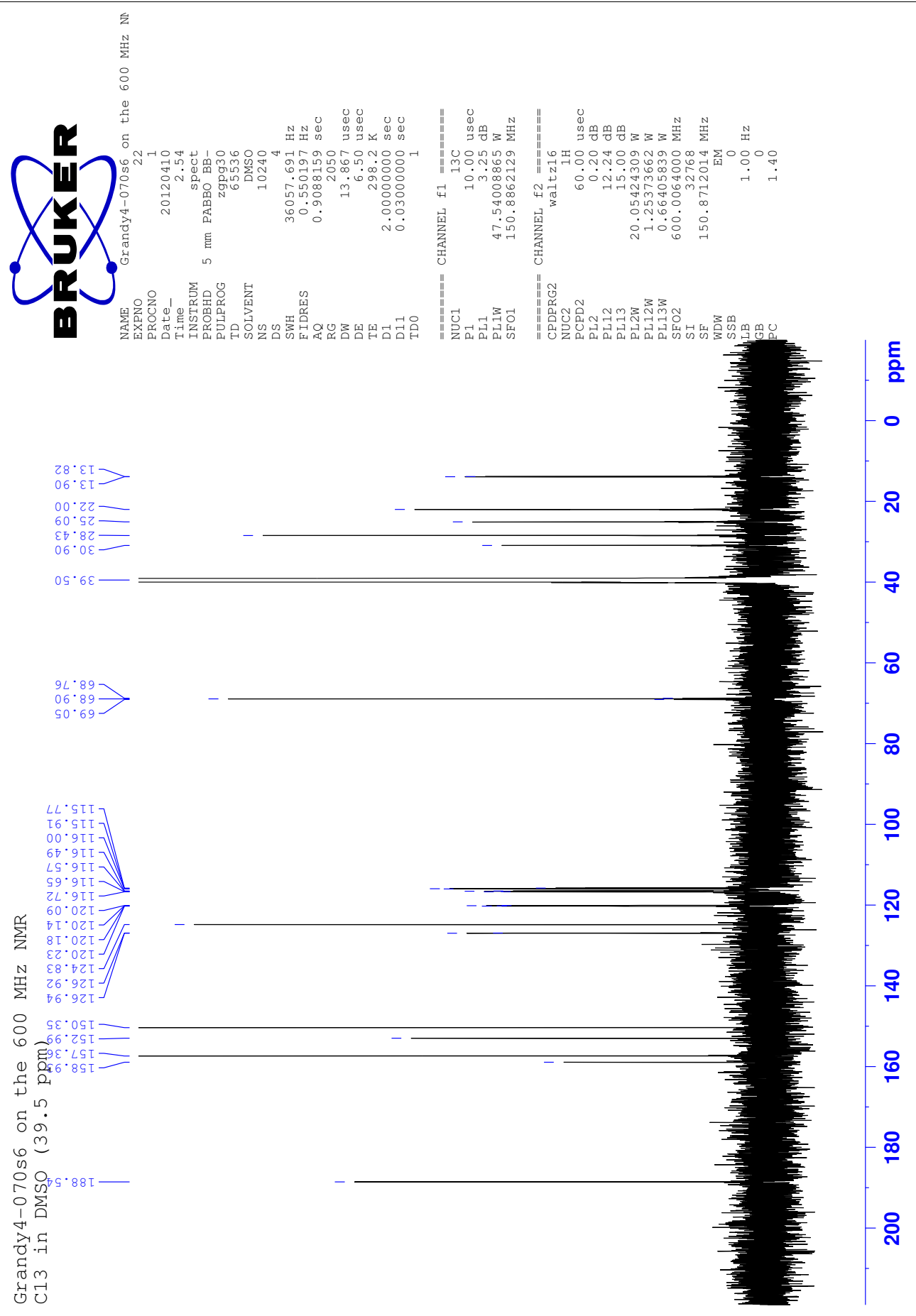


5-(4-fluorophenoxy)-2-(hexyloxy)benzaldehyde: G4-068s20 \& G4-070s6

FIGURE A.12: HSQC-NMR of G4-070s6.

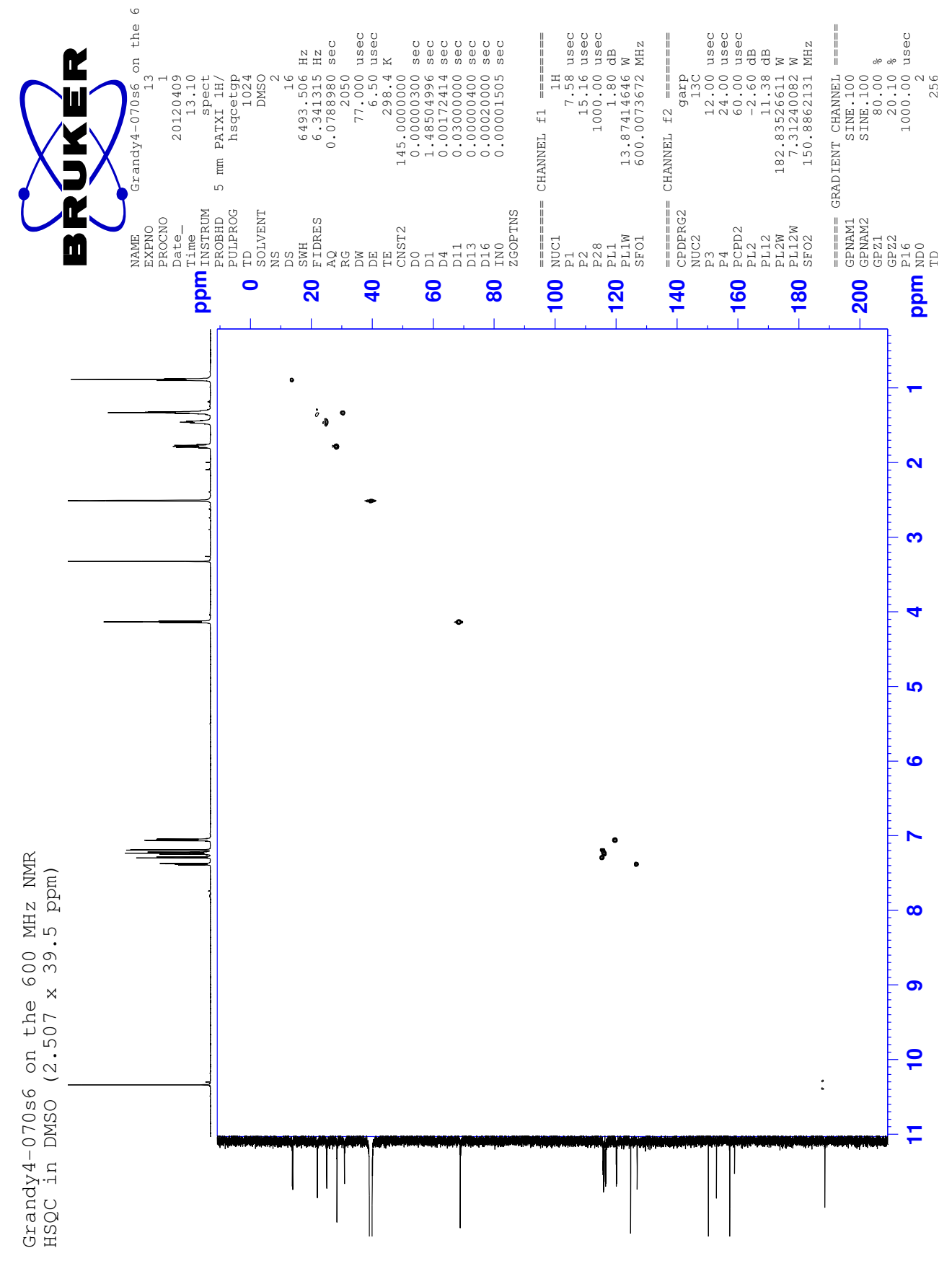


5-(4-fluorophenoxy)-2-(hexyloxy)benzaldehyde: G4-068s20 \& G4-070s6

FIGURE A.13: HMBC-NMR of G4-070s6.

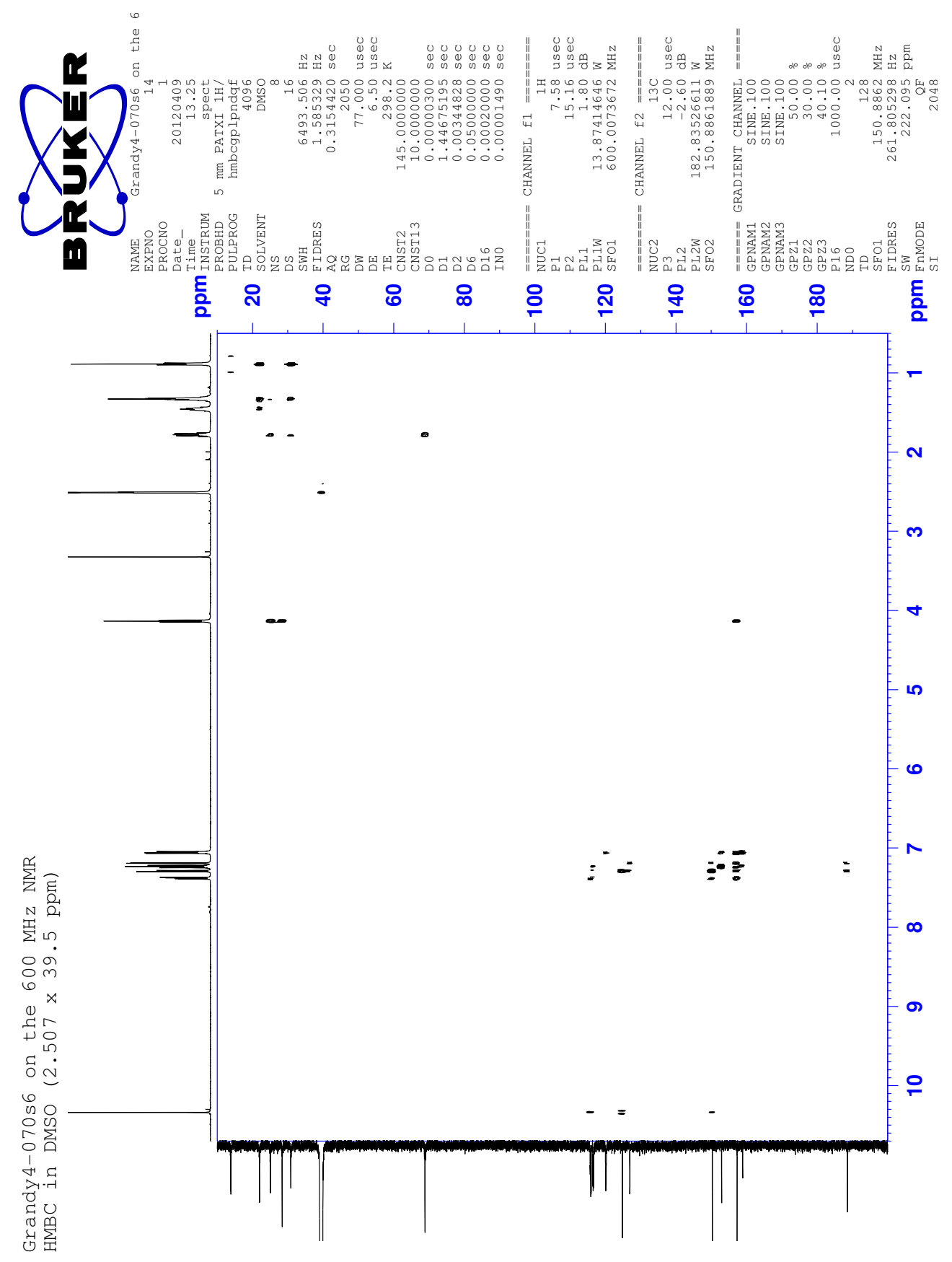


5-(4-fluorophenoxy)-2-(hexyloxy)benzaldehyde: G4-068s20 \& G4-070s6

FIGURE A.14: NOESY-NMR of G4-070s6.

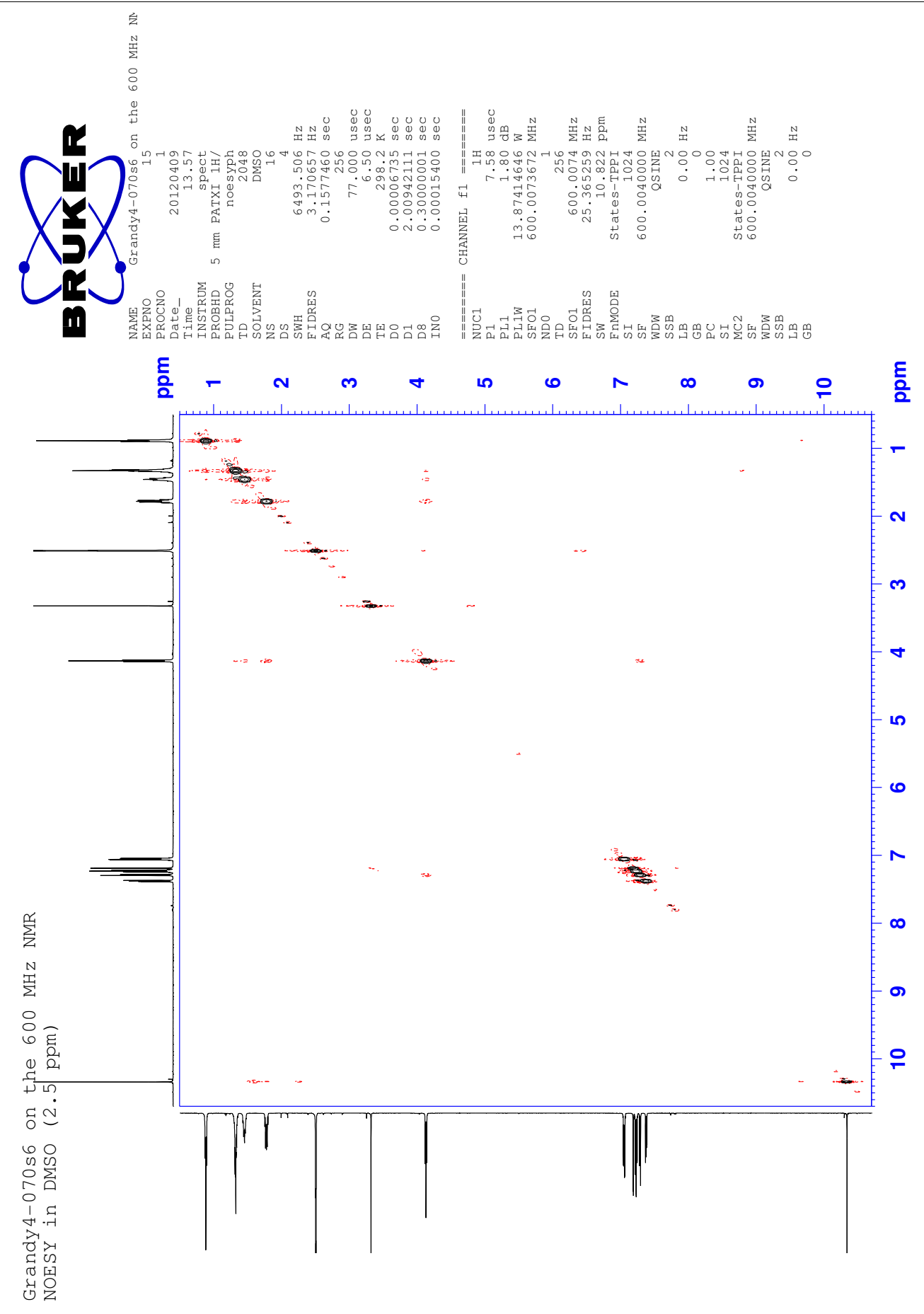


5-(4-fluorophenoxy)-2-(hexyloxy)benzaldehyde: G4-068s20 \& G4-070s6

FIGURE A.15: ${ }^{19} \mathrm{~F}-\mathrm{NMR}$ of G4-070s6, $400 \mathrm{MHz}$ with $\mathrm{C}_{6} \mathrm{~F}_{6}$ (-164.0 ppm).
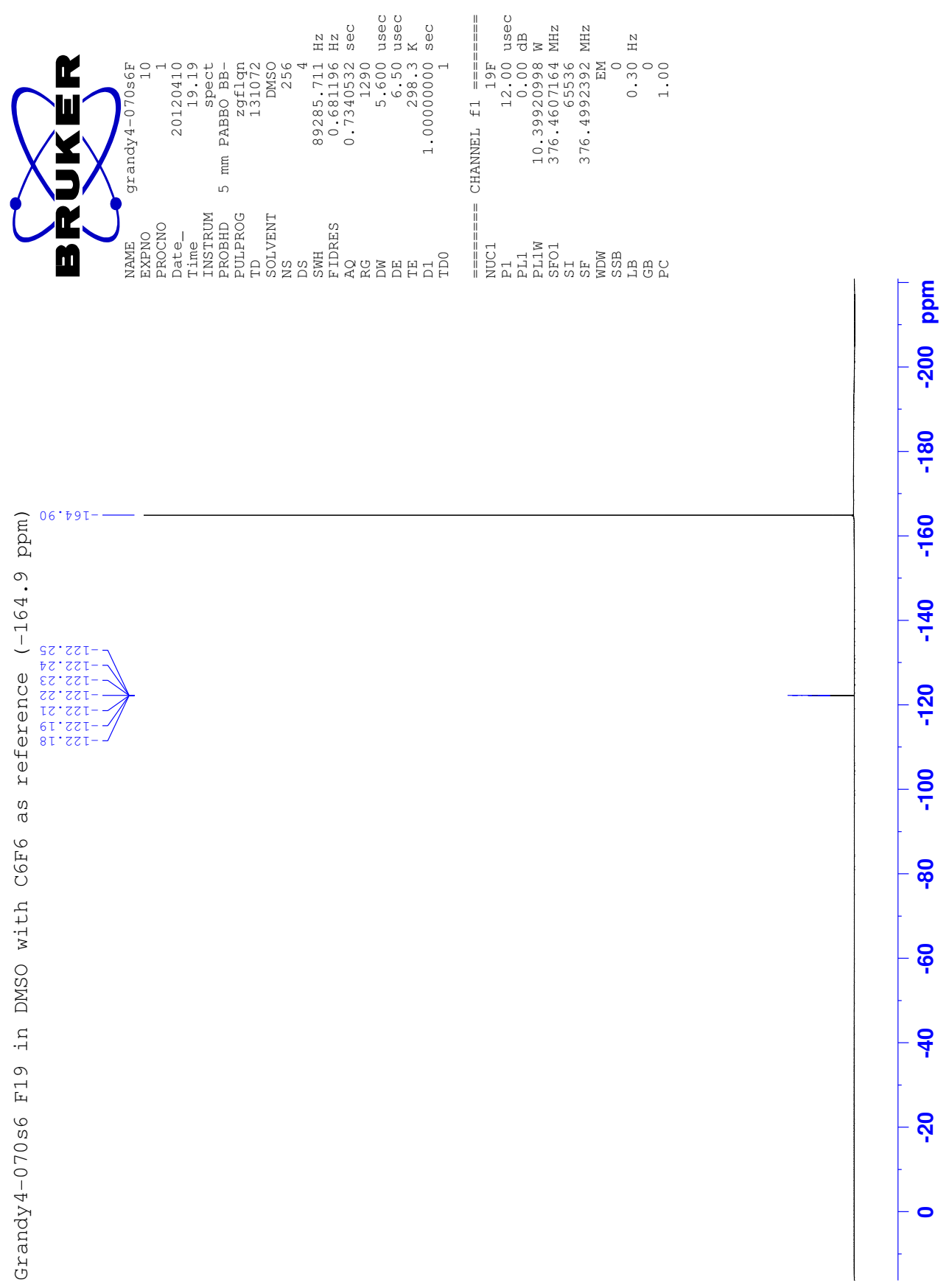
(5-(4-fluorophenoxy)-2-(hexyloxy)phenyl)(phenyl)methanol: G4-074s14

\section{A.3 (5-(4-fluorophenoxy)-2-(hexyloxy)phenyl)(phenyl)methanol: G4-074s14}

FIGURE A.16: Structure of G4-074s14.

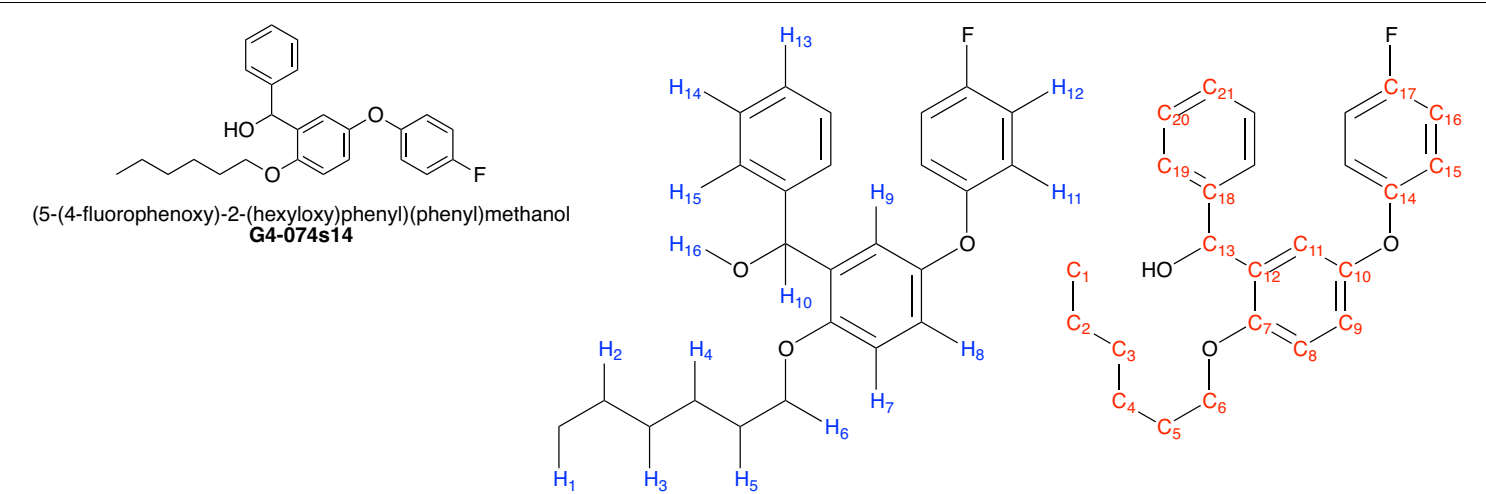

TABLE A.5: ${ }^{1} \mathrm{H}-\mathrm{NMR}$ assignments for G4-074s14, $600 \mathrm{MHz}$ in DMSO (2.51 ppm)

\begin{tabular}{|c|c|c|c|c|c|}
\hline Proton & $\mathbf{p p m}^{\mathbf{a}}$ & Number & Type & $\mathbf{J}_{\mathbf{a}}$ & $\mathbf{J}_{\mathbf{b}}$ \\
\hline $\mathbf{H}_{1}$ & 0.881 & 3 & $\mathbf{t}$ & 7.2 & \\
\hline $\mathrm{H}_{2} \& \mathrm{H}_{3}$ & 1.289 & 4 & $\mathbf{m}$ & & \\
\hline $\mathbf{H}_{4}$ & 1.354 & 2 & $\mathbf{m}$ & & \\
\hline $\mathrm{H}_{5}$ & 1.660 & 2 & $\mathbf{m}$ & & \\
\hline $\mathrm{H}_{6}$ & 3.888 & 2 & $\mathbf{m}$ & & \\
\hline $\mathbf{H}_{7}$ & 6.922 & 1 & d & 9.0 & \\
\hline $\mathrm{H}_{8}$ & 6.848 & 1 & dd & 9.0 & 3.0 \\
\hline $\mathrm{H}_{9}$ & 7.228 & 1 & d & 3.0 & \\
\hline $\mathrm{H}_{10}$ & 5.912 & 1 & d & 9.3 & \\
\hline $\mathrm{H}_{11}$ & 7.197 & 2 & $\mathbf{m}$ & & \\
\hline $\mathrm{H}_{12}$ & 6.974 & 2 & $\mathbf{m}$ & & \\
\hline $\mathrm{H}_{13}$ & 7.186 & 2 & $\mathbf{m}$ & & \\
\hline $\mathrm{H}_{14}$ & 7.287 & 2 & $\mathbf{m}$ & & \\
\hline $\mathrm{H}_{15}$ & 7.265 & 2 & $\mathbf{m}$ & & \\
\hline $\mathrm{H}_{16}$ & 5.730 & 1 & d & & \\
\hline
\end{tabular}

a. Taken at the center of the peak.
TABLE A.6: ${ }^{13} \mathrm{C}-\mathrm{NMR}$ assignments for G4-074s14, $600 \mathrm{MHz}$ in DMSO (39.51 ppm)

\begin{tabular}{|c|c|c|c|c|c|}
\hline Carbon & ppm $^{\mathbf{a}}$ & Carbon & $\mathbf{p p m}^{\mathbf{a}}$ & Type $^{\text {b }}$ & $\mathbf{J}$ \\
\hline$C_{1}$ & 13.87 & $\mathrm{C}_{12}$ & 135.38 & & \\
\hline $\mathrm{C}_{2}$ & 22.03 & $\mathrm{C}_{13}$ & 68.22 & & \\
\hline$C_{3}$ & 30.93 & $\mathrm{C}_{14}$ & 153.94 & & \\
\hline $\mathrm{C}_{4}$ & 25.22 & $\mathrm{C}_{15}$ & 119.20 & d & 7.5 \\
\hline $\mathrm{C}_{5}$ & 28.69 & $\mathrm{C}_{16}$ & 116.33 & d & 22.6 \\
\hline $\mathrm{C}_{6}$ & 67.90 & $\mathrm{C}_{17}$ & 157.64 & d & 239.9 \\
\hline $\mathrm{C}_{7}$ & 151.09 & $\mathrm{C}_{18}$ & 144.80 & & \\
\hline $\mathrm{C}_{8}$ & 112.58 & $\mathrm{C}_{19}$ & 126.65 & & \\
\hline $\mathrm{C}_{9}$ & 117.92 & $\mathrm{C}_{20}$ & 127.82 & & \\
\hline $\mathrm{C}_{10}$ & 149.73 & $C_{21}$ & 126.54 & & \\
\hline $\mathrm{C}_{11}$ & 117.16 & & & & \\
\hline
\end{tabular}

a. Taken at the center of the peak.

b. Singlet unless noted otherwise. 
(5-(4-fluorophenoxy)-2-(hexyloxy)phenyl)(phenyl)methanol: G4-074s14

FIGURE A.17: ${ }^{1} \mathrm{H}-\mathrm{NMR}$ of G4-074s14.

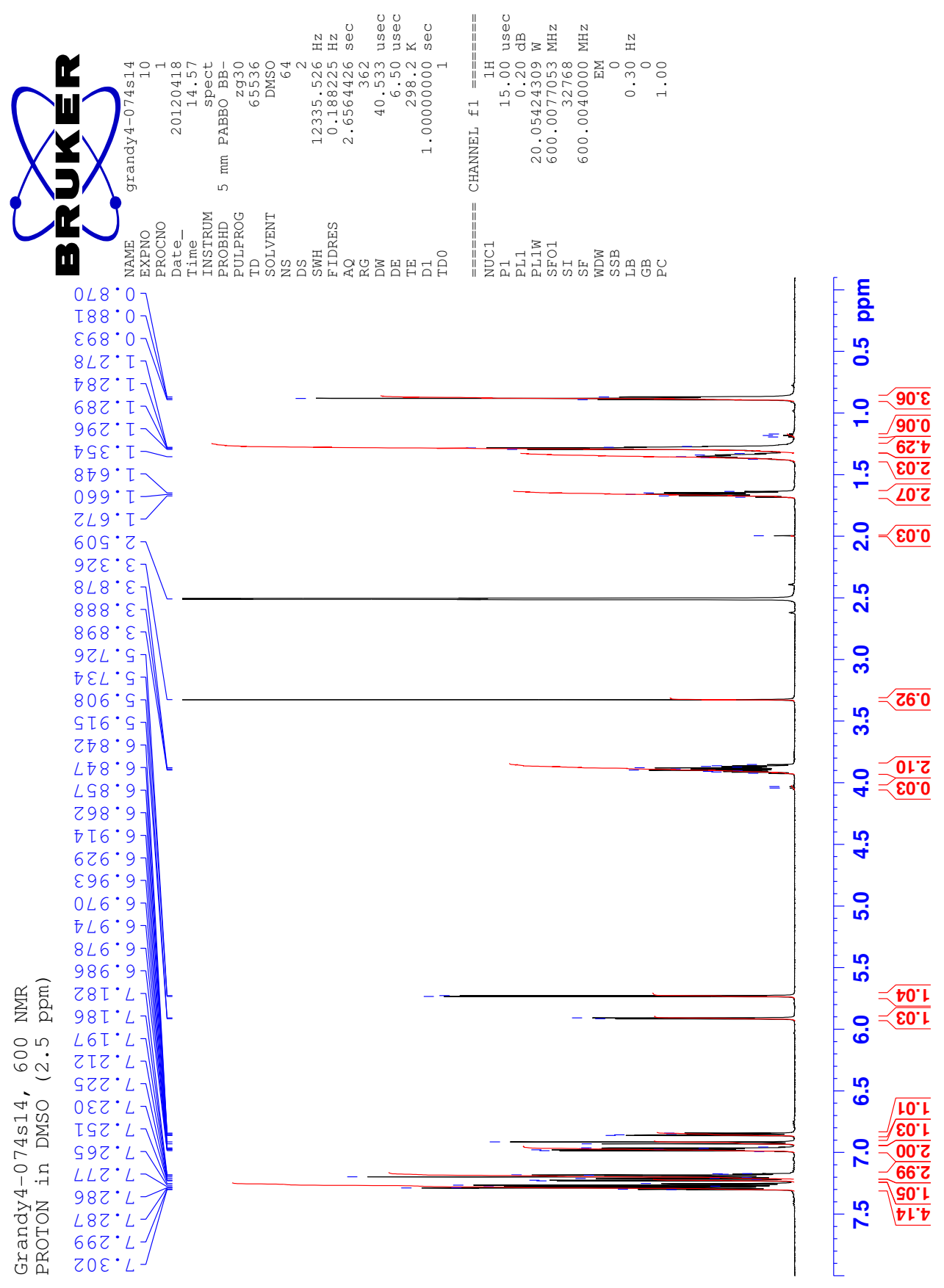


(5-(4-fluorophenoxy)-2-(hexyloxy)phenyl)(phenyl)methanol: G4-074s14

FIGURE A.18: COSY-NMR of G4-074s14.

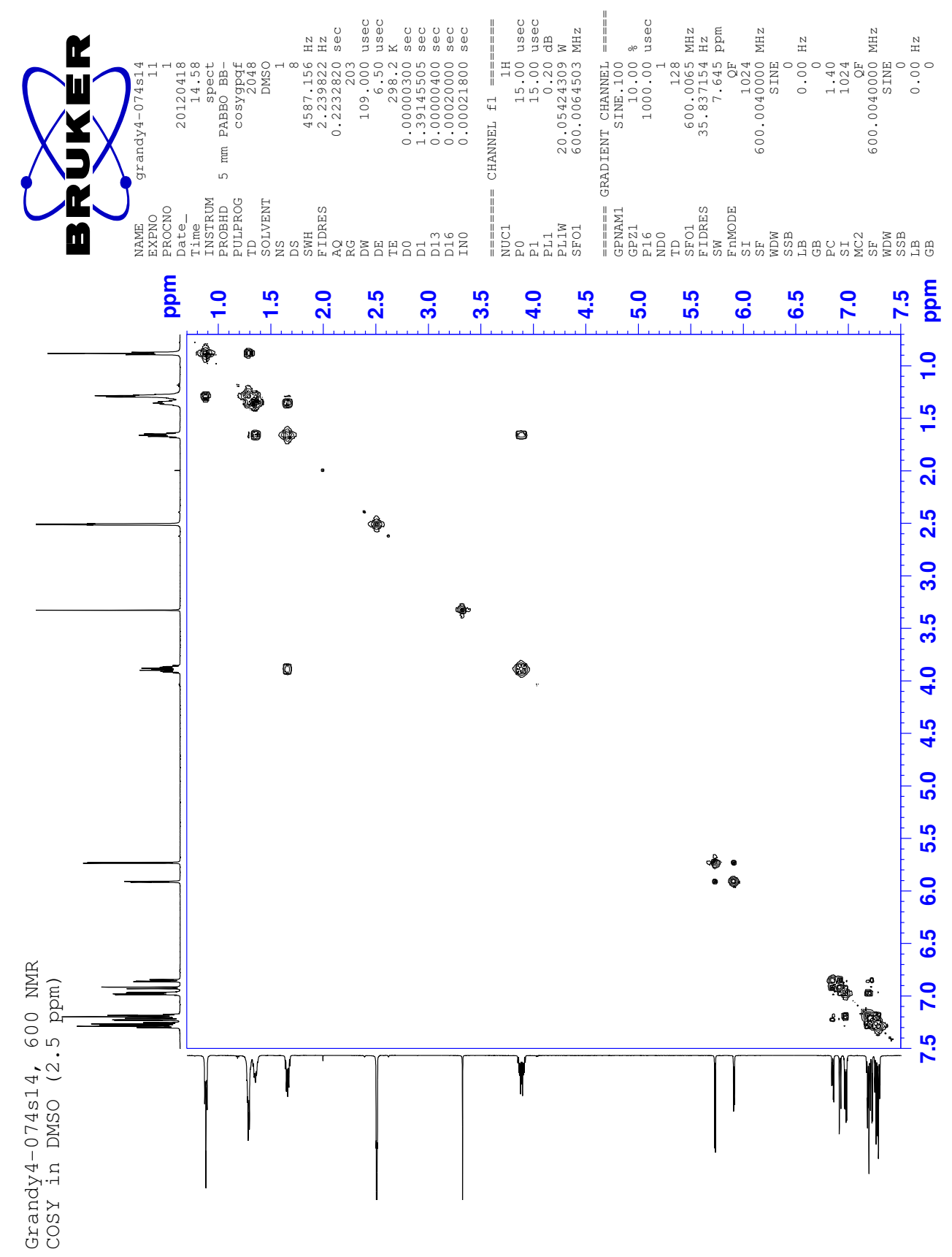


(5-(4-fluorophenoxy)-2-(hexyloxy)phenyl)(phenyl)methanol: G4-074s14

FIGURE A.19: ${ }^{13} \mathrm{C}-\mathrm{NMR}$ of G4-074s14.

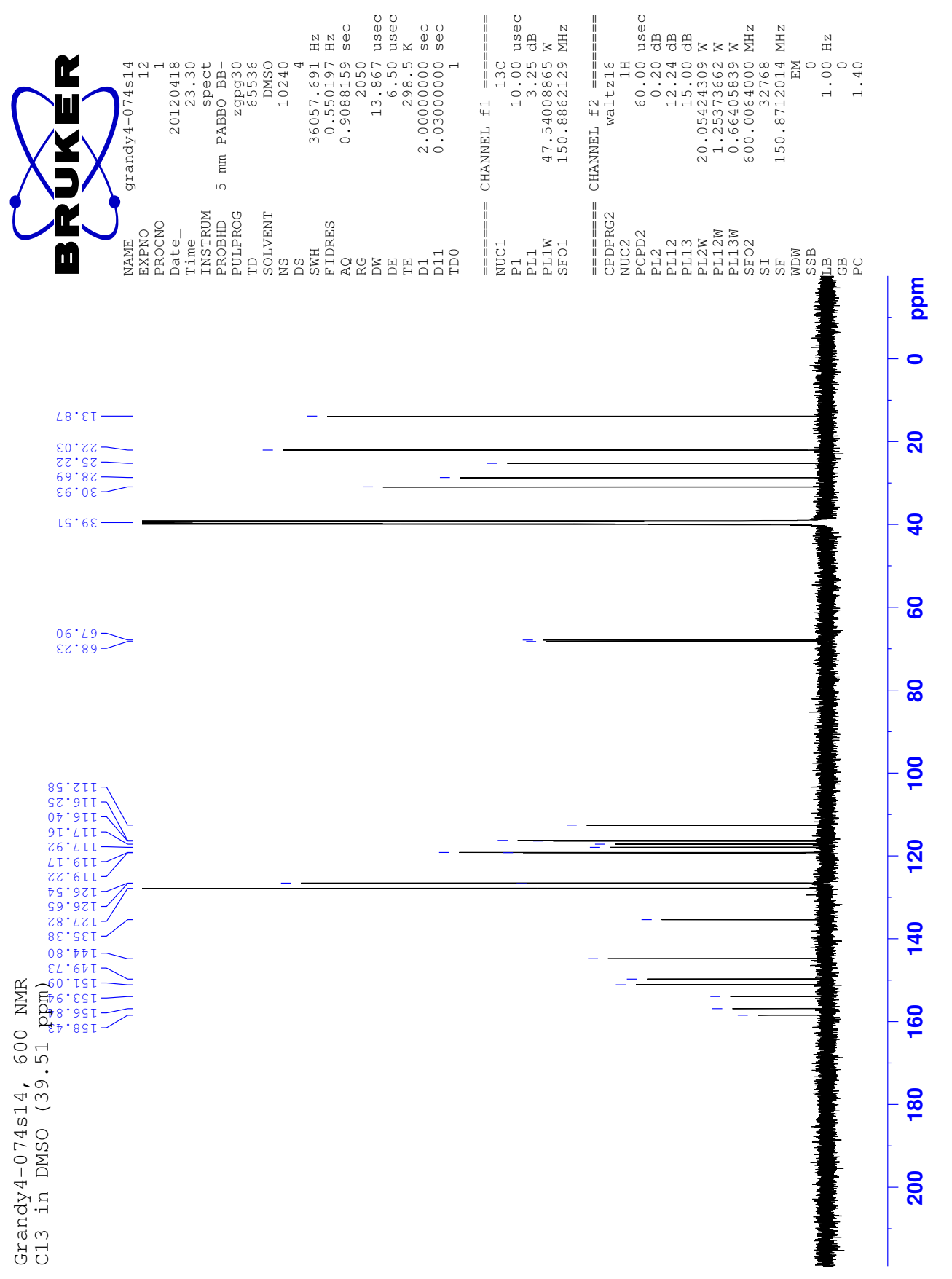


(5-(4-fluorophenoxy)-2-(hexyloxy)phenyl)(phenyl)methanol: G4-074s14

FIGURE A.20: HSQC-NMR of G4-074s14.

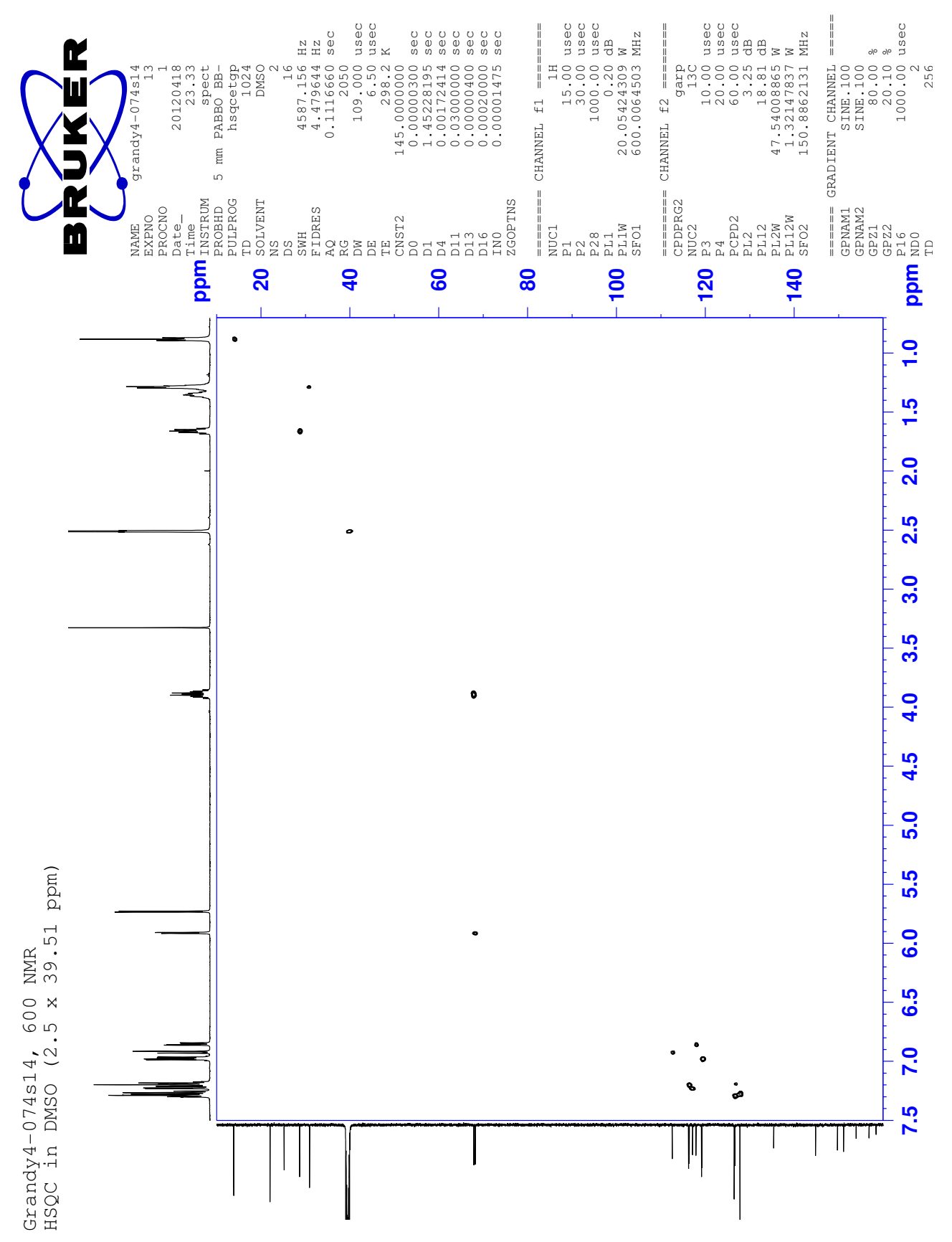


(5-(4-fluorophenoxy)-2-(hexyloxy)phenyl)(phenyl)methanol: G4-074s14

FIGURE A.21: HMBC-NMR of G4-074s14.

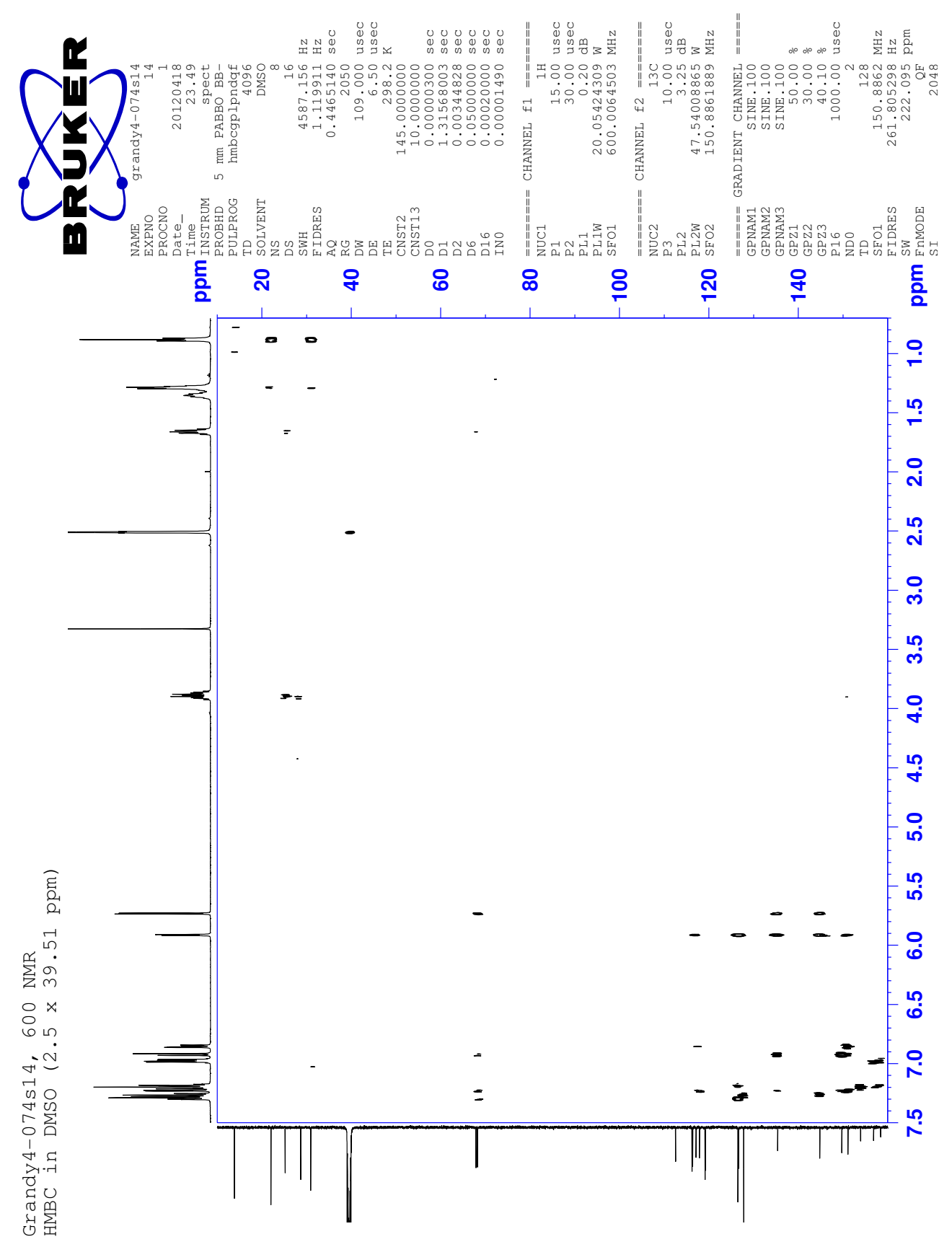


(5-(4-fluorophenoxy)-2-(hexyloxy)phenyl)(phenyl)methanol: G4-074s14

FIGURE A.22: NOESY-NMR of G4-074s14.

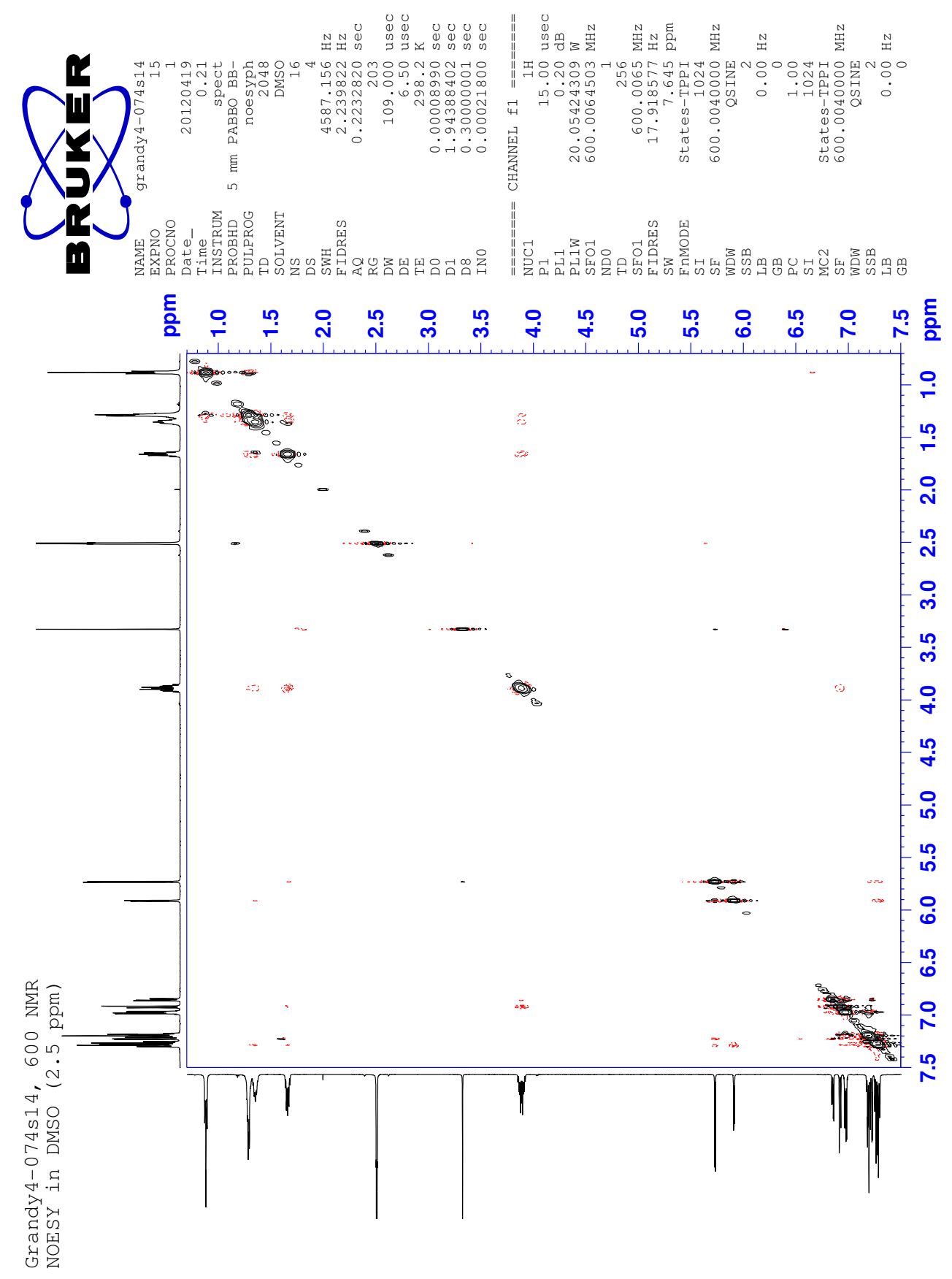


(5-(4-fluorophenoxy)-2-(hexyloxy)phenyl)(phenyl)methanol: G4-074s14

FIGURE A.23: ${ }^{19} \mathrm{~F}-\mathrm{NMR}$ of G4-074s14, $400 \mathrm{MHz}$ with $\mathrm{C}_{6} \mathrm{~F}_{6}(-164.0 \mathrm{ppm})$.
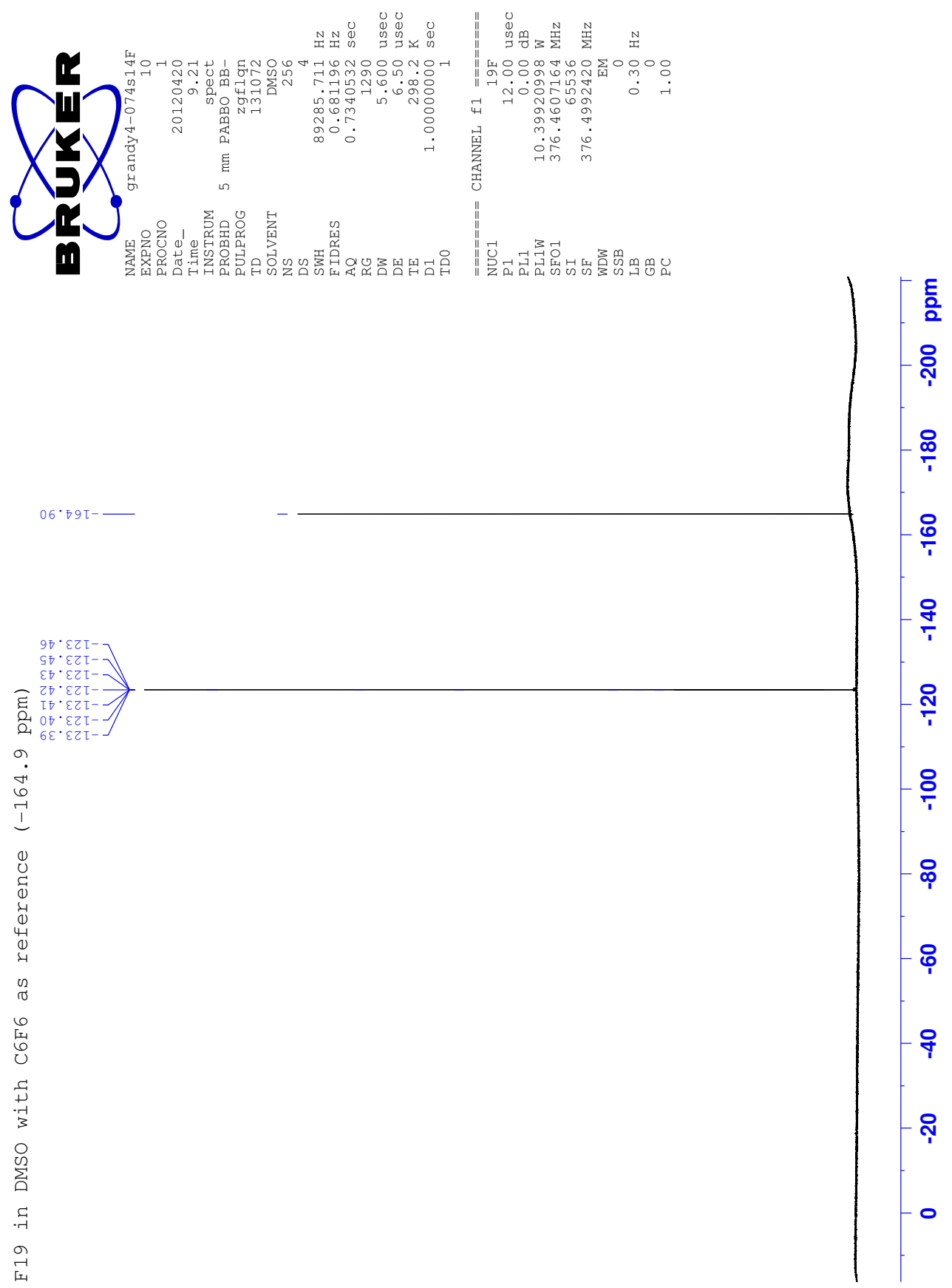
2-(5-(4-fluorophenoxy)-2-(hexyloxy)phenyl)-2-phenylacetonitrile: G4-079s9

A.4 2-(5-(4-fluorophenoxy)-2-(hexyloxy)phenyl)-2-phenylacetonitrile: G4-079s9

FIGURE A.24: Structure of G4-079s9.

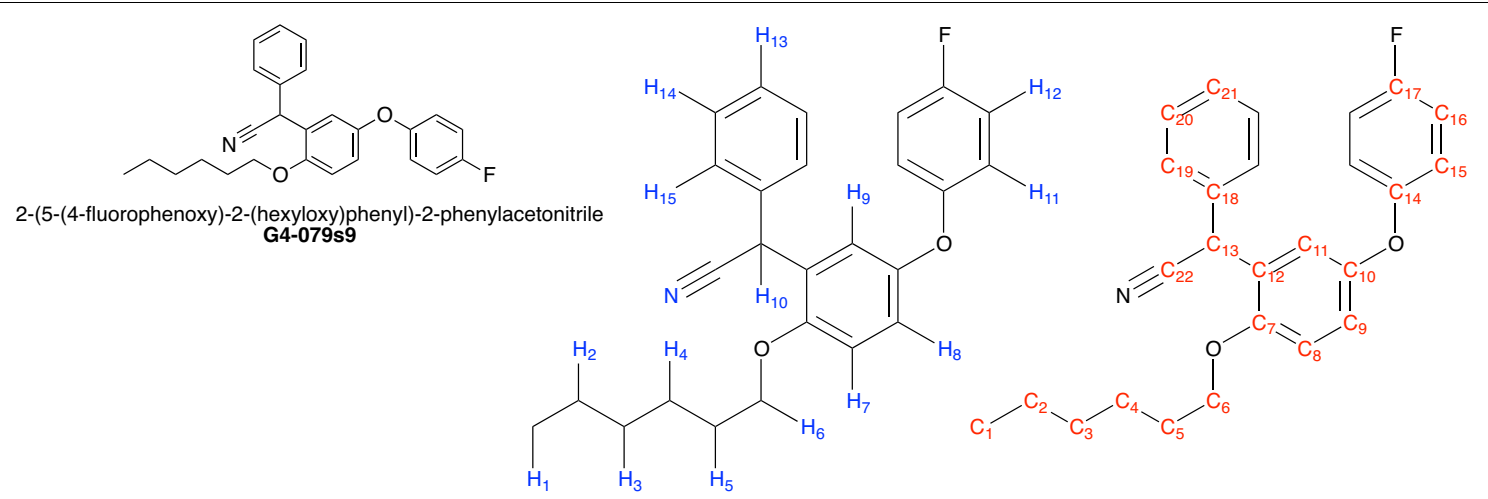

TABLE A.7: ${ }^{1} \mathrm{H}-\mathrm{NMR}$ assignments for G4-079s9, $600 \mathrm{MHz}$ in DMSO (2.51 ppm)

\begin{tabular}{|c|c|c|c|c|c|}
\hline Proton & $\mathbf{p p m}^{\mathbf{a}}$ & Number & Type & $\mathbf{J}_{\mathbf{a}}$ & $\mathbf{J}_{\mathbf{b}}$ \\
\hline $\mathbf{H}_{1}$ & 0.874 & 3 & $\mathbf{t}$ & 7.5 & \\
\hline $\mathbf{H}_{2} \& \mathbf{H}_{3}$ & 1.269 & 4 & m & & \\
\hline $\mathbf{H}_{4}$ & 1.331 & 2 & m & & \\
\hline $\mathbf{H}_{5}$ & 1.644 & 2 & m & & \\
\hline $\mathbf{H}_{6}$ & 3.929 & 2 & m & & \\
\hline $\mathbf{H}_{7}$ & 7.060 & 1 & d & 9.0 & \\
\hline $\mathbf{H}_{8}$ & 6.994 & 1 & dd & 9.0 & 3.0 \\
\hline $\mathbf{H}_{9}$ & 7.094 & 1 & d & 3.0 & \\
\hline $\mathbf{H}_{10}$ & 5.748 & 1 & $\mathbf{s}$ & & \\
\hline $\mathbf{H}_{11}$ & 7.210 & 2 & $\mathbf{m}$ & & \\
\hline $\mathrm{H}_{12}$ & 7.015 & 2 & m & & \\
\hline $\mathrm{H}_{13}$ & 7.320 & 2 & m & & \\
\hline $\mathrm{H}_{14}$ & 7.385 & 2 & $\mathbf{m}$ & & \\
\hline $\mathrm{H}_{15}$ & 7.357 & 2 & m & & \\
\hline
\end{tabular}

a. Taken at the center of the peak.
TABLE A.8: ${ }^{13} \mathrm{C}-\mathrm{NMR}$ assignments for G4-079s9, $600 \mathrm{MHz}$ in DMSO (39.52 ppm)

\begin{tabular}{|c|c|c|c|c|c|}
\hline Carbon & ppm $^{a}$ & Carbon & ppm $^{\mathbf{a}}$ & Type $^{\mathbf{b}}$ & $\mathbf{J}$ \\
\hline $\mathrm{C}_{1}$ & 13.86 & $\mathrm{C}_{12}$ & 125.37 & & \\
\hline $\mathrm{C}_{2}$ & 21.99 & $\mathrm{C}_{13}$ & 36.61 & & \\
\hline $\mathrm{C}_{3}$ & 30.94 & $\mathrm{C}_{14}$ & 153.47 & d & 1.5 \\
\hline $\mathrm{C}_{4}$ & 25.04 & $\mathrm{C}_{15}$ & 119.53 & d & 7.5 \\
\hline $\mathrm{C}_{5}$ & 28.50 & $\mathrm{C}_{16}$ & 116.47 & d & 22.6 \\
\hline $\mathrm{C}_{6}$ & 68.33 & $\mathrm{C}_{17}$ & 157.88 & d & 239.9 \\
\hline $\mathrm{C}_{7}$ & 151.86 & $\mathrm{C}_{18}$ & 135.37 & & \\
\hline $\mathrm{C}_{8}$ & 113.67 & $\mathrm{C}_{19}$ & 127.41 & & \\
\hline $\mathrm{C}_{9}$ & 119.76 & $\mathbf{C}_{20}$ & 128.76 & & \\
\hline $\mathrm{C}_{10}$ & 149.74 & $\mathrm{C}_{21}$ & 127.75 & & \\
\hline $\mathrm{C}_{11}$ & 119.79 & $\mathrm{C}_{22}$ & 119.62 & & \\
\hline
\end{tabular}

a. Taken at the center of the peak.

b. Singlet unless noted otherwise. 
2-(5-(4-fluorophenoxy)-2-(hexyloxy)phenyl)-2-phenylacetonitrile: G4-079s9

FIGURE A.25: ${ }^{1} \mathrm{H}-\mathrm{NMR}$ of G4-079s9.

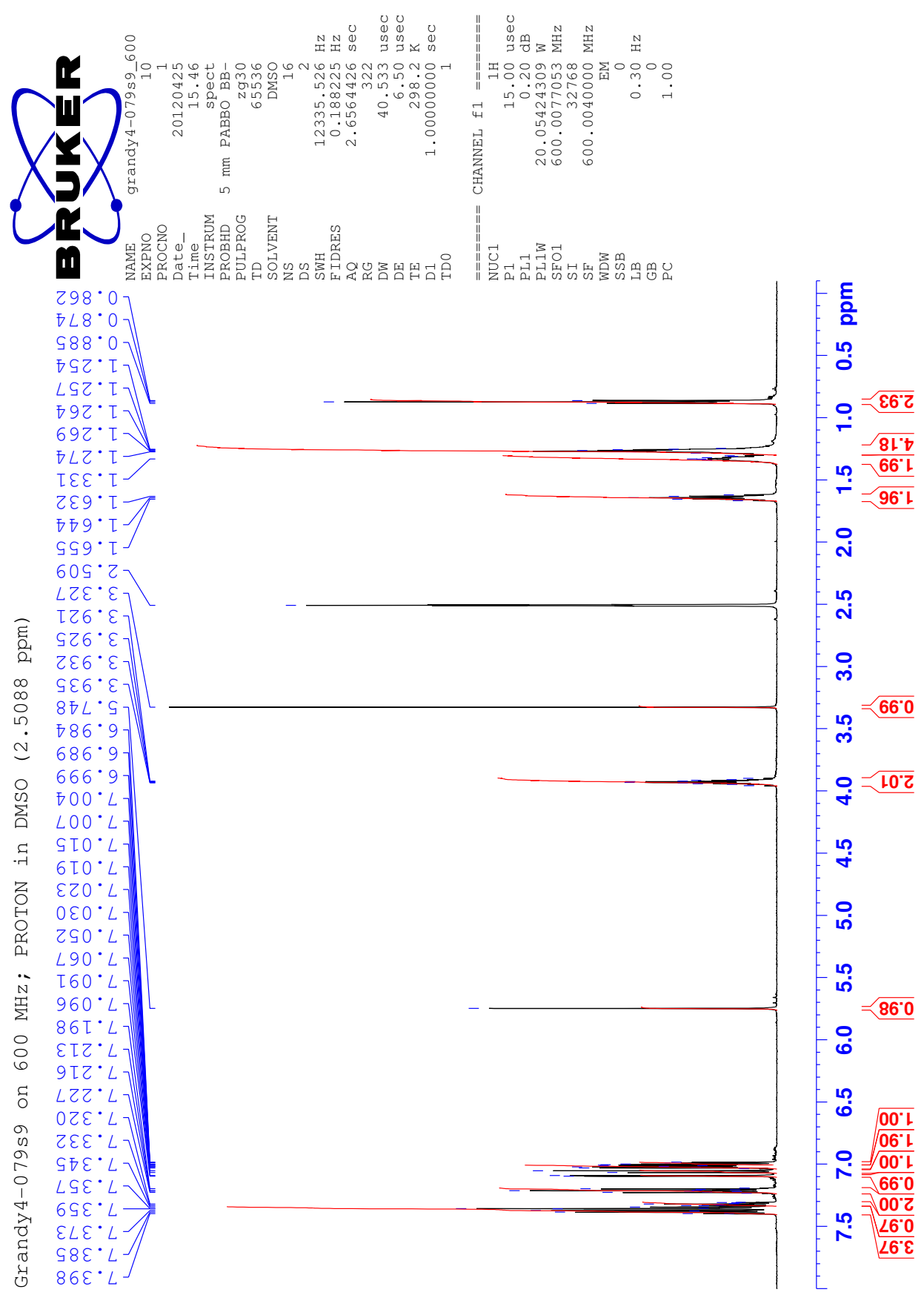


2-(5-(4-fluorophenoxy)-2-(hexyloxy)phenyl)-2-phenylacetonitrile: G4-079s9

FIGURE A.26: COSY-NMR of G4-079s9.

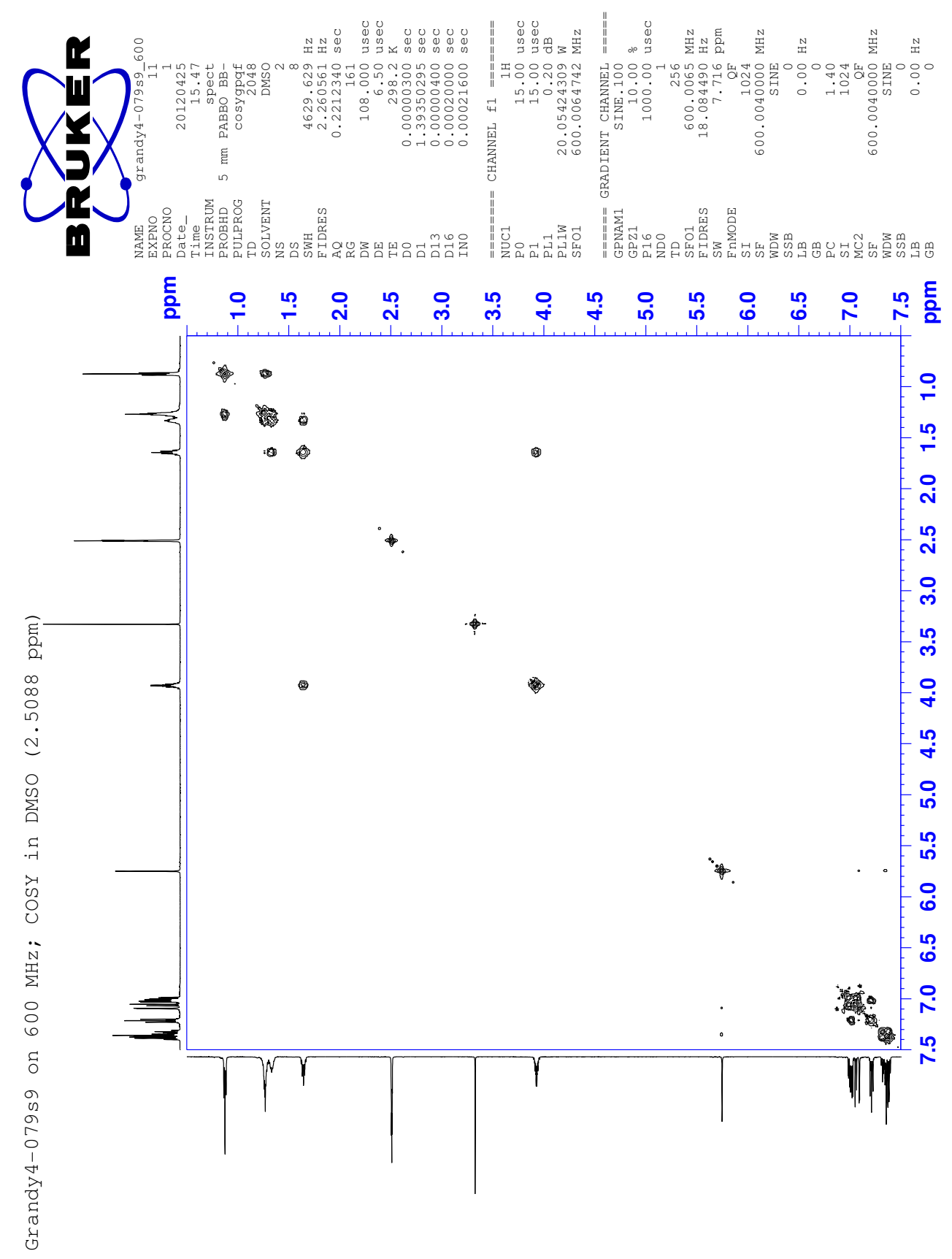


2-(5-(4-fluorophenoxy)-2-(hexyloxy)phenyl)-2-phenylacetonitrile: G4-079s9

FIGURE A.27: ${ }^{13} \mathrm{C}-\mathrm{NMR}$ of G4-079s9.

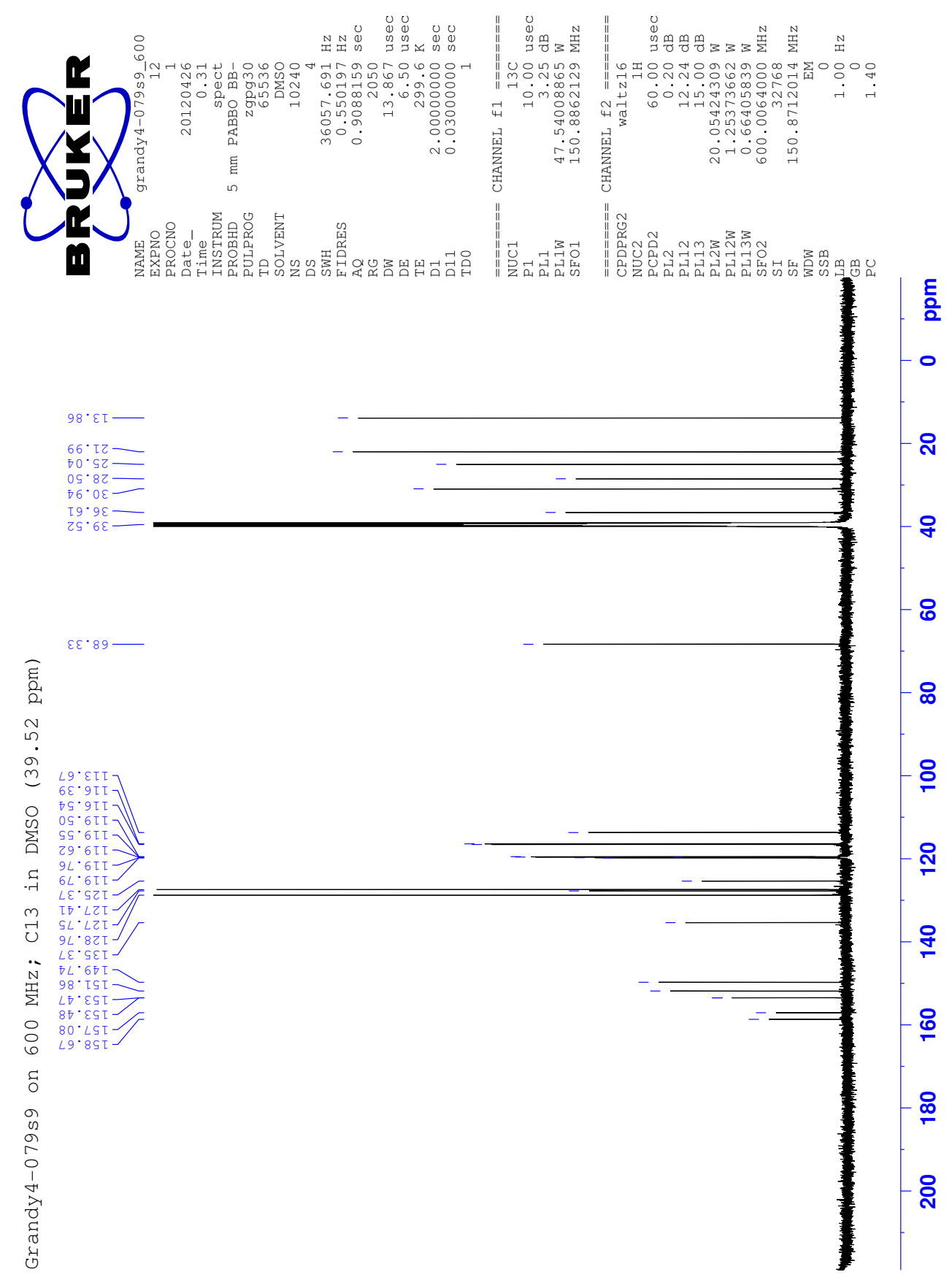


2-(5-(4-fluorophenoxy)-2-(hexyloxy)phenyl)-2-phenylacetonitrile: G4-079s9

FIGURE A.28: HSQC-NMR of G4-079s9.

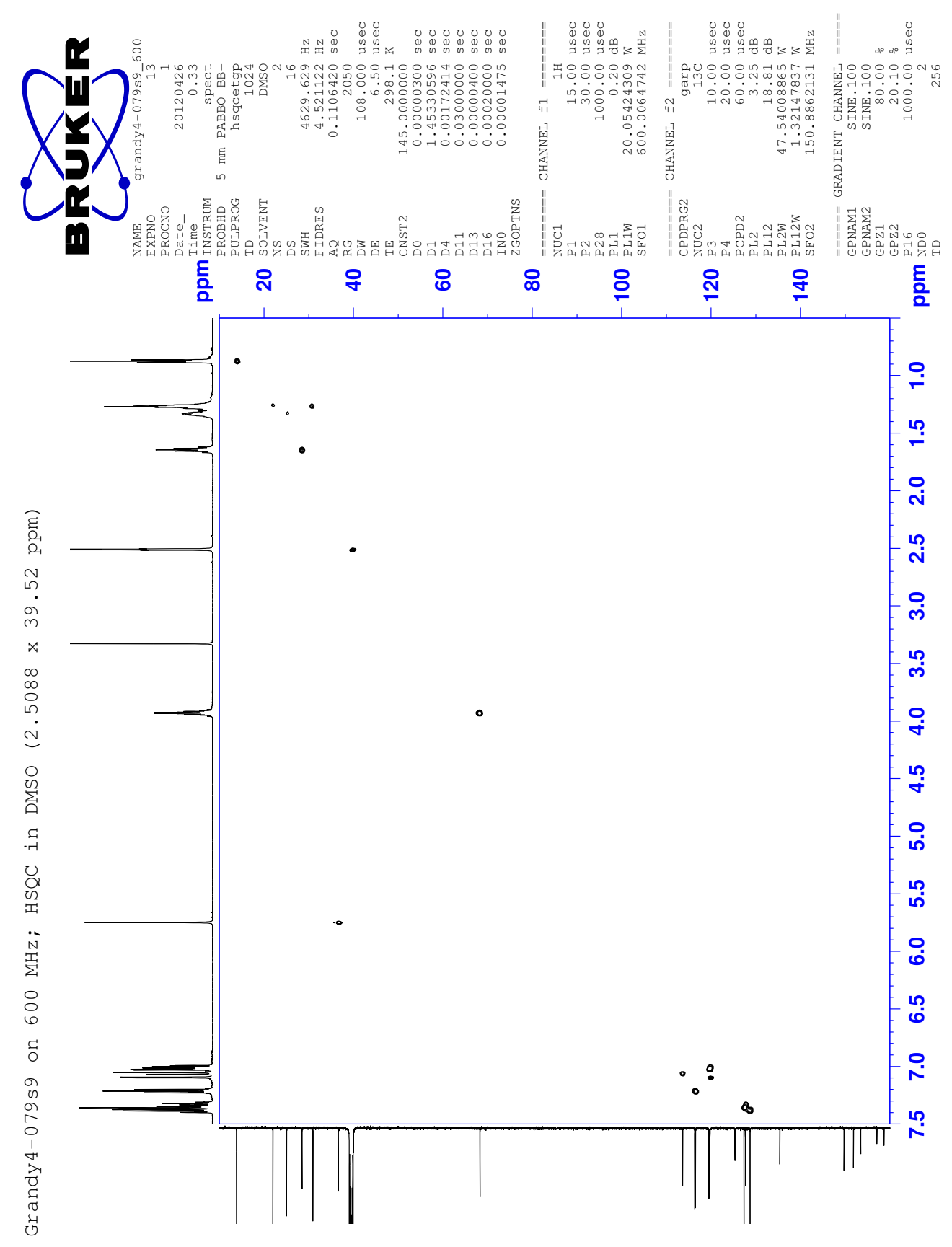


2-(5-(4-fluorophenoxy)-2-(hexyloxy)phenyl)-2-phenylacetonitrile: G4-079s9

FIGURE A.29: HMBC-NMR of G4-079s9.

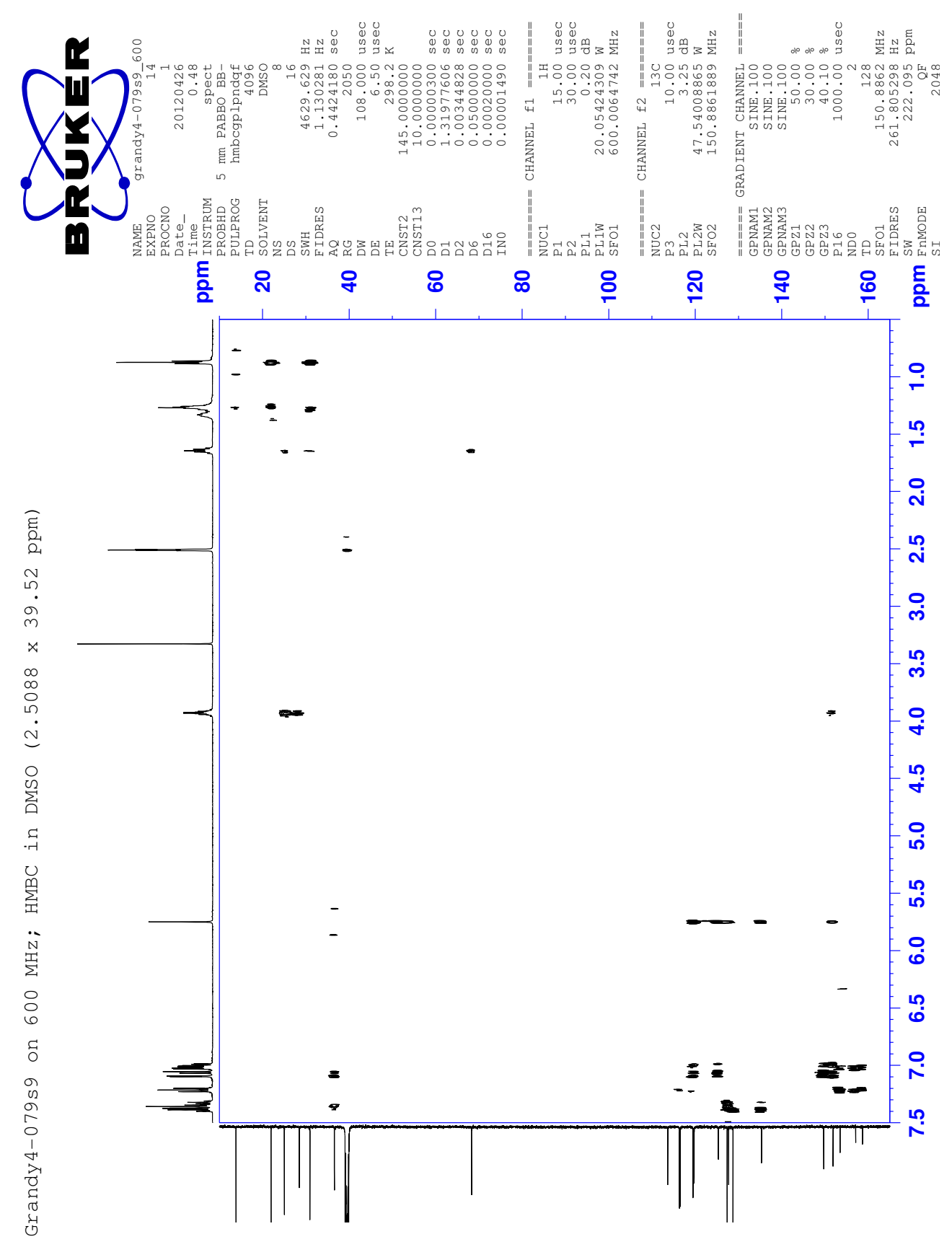


2-(5-(4-fluorophenoxy)-2-(hexyloxy)phenyl)-2-phenylacetonitrile: G4-079s9

FIGURE A.30: NOESY-NMR of G4-079s9.

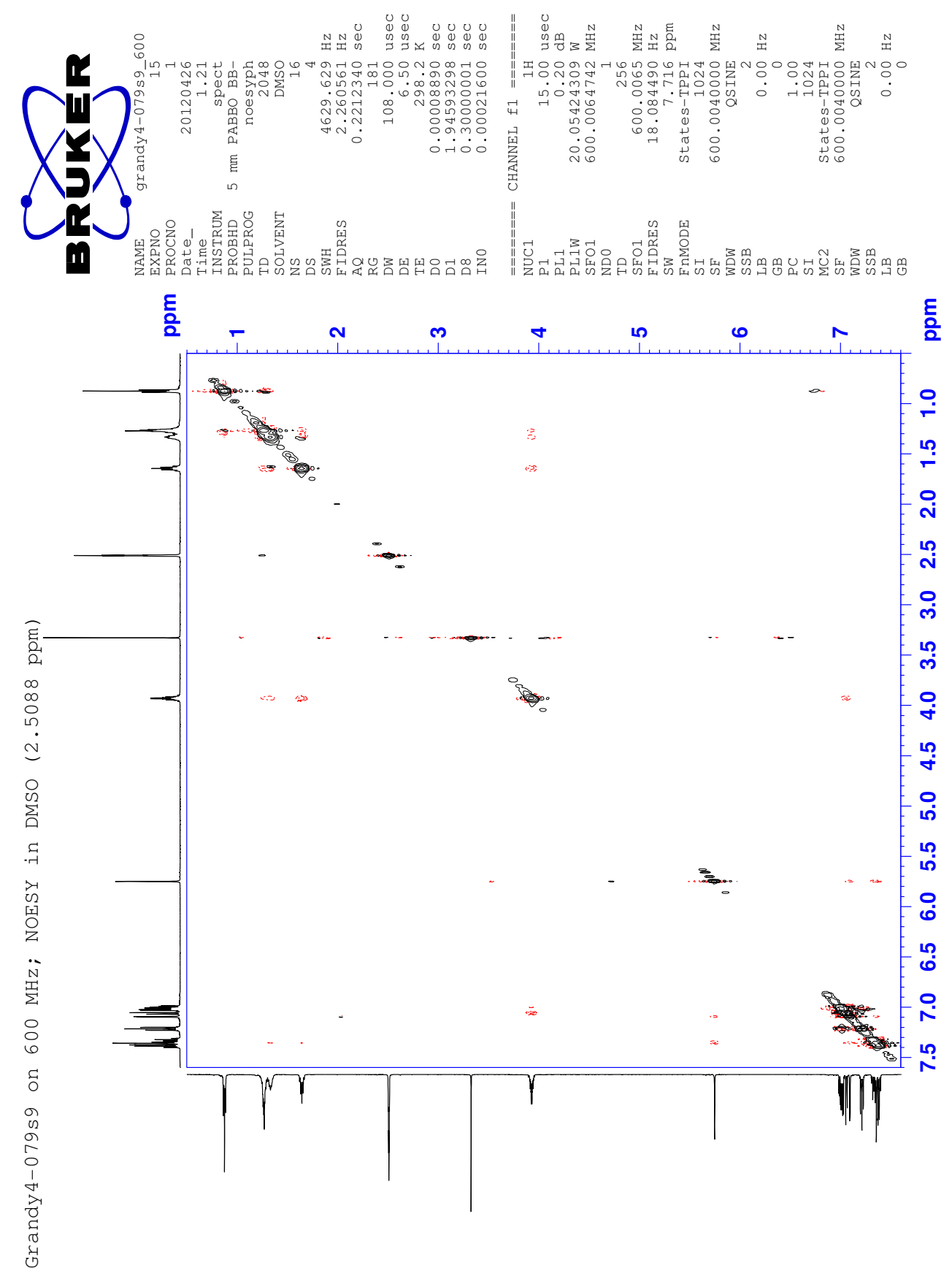


2-(5-(4-fluorophenoxy)-2-(hexyloxy)phenyl)-2-phenylacetonitrile: G4-079s9

FIGURE A.31: ${ }^{19}$ F-NMR of G4-079s9, $400 \mathrm{MHz}$ with $\mathrm{C}_{6} \mathrm{~F}_{6}(-164.0 \mathrm{ppm})$.
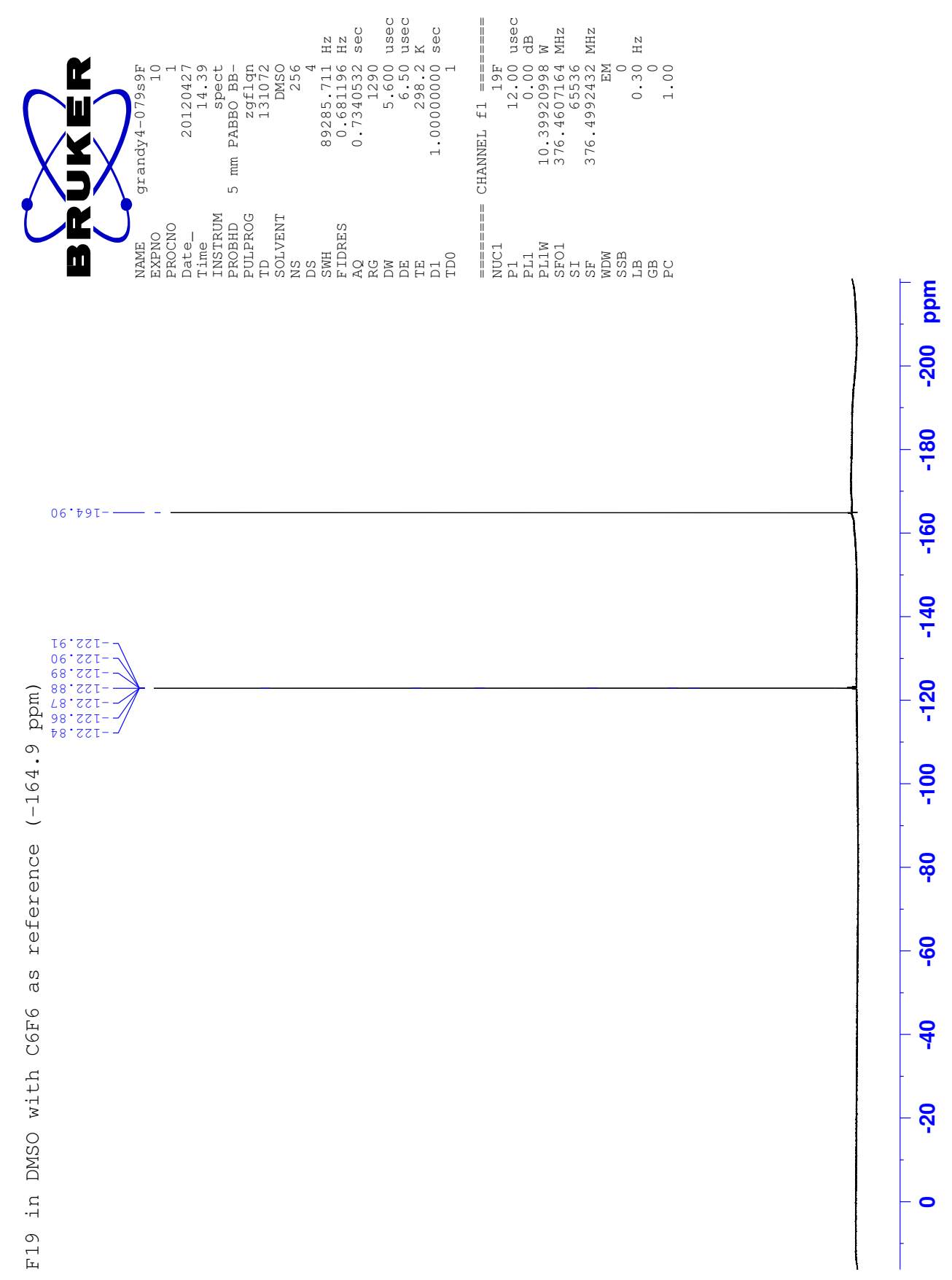
ET-92: 2-(5-(4-fluorophenoxy)-2-(hexyloxy)phenyl)-2-phenylethanamine hydrochlo-

\section{A.5 ET-92: 2-(5-(4-fluorophenoxy)-2-(hexyloxy)phenyl)-2-phenylethanamine} hydrochloride: G4-091s14

FIGURE A.32: Structure of ET-92 (G4-090s14).

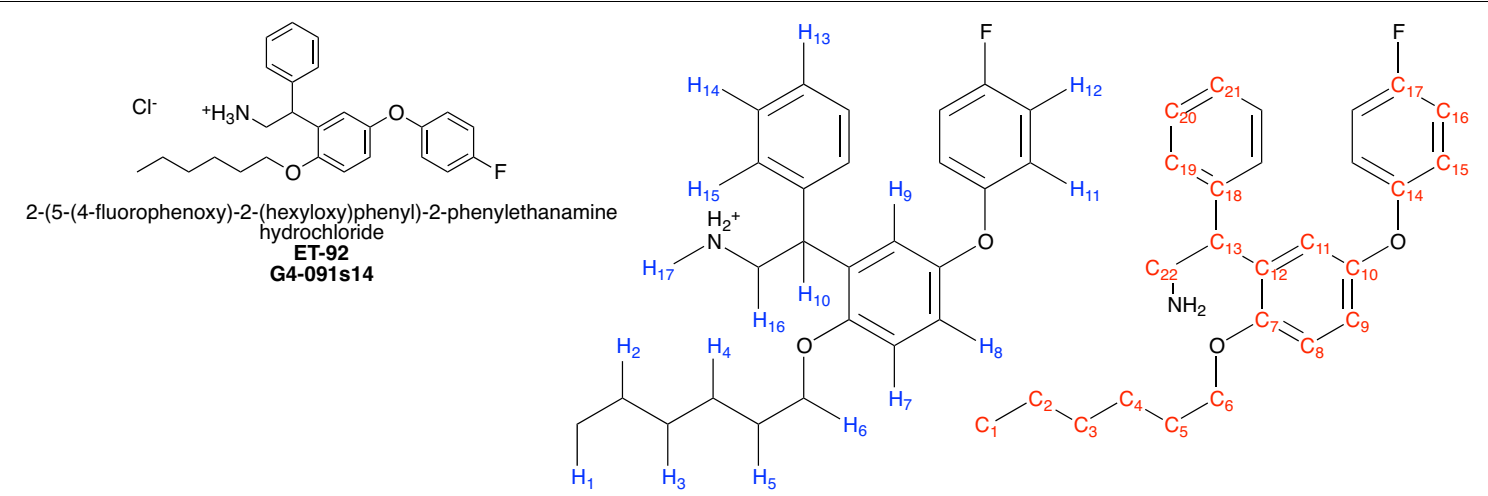

TABLE A.9: ${ }^{1} \mathrm{H}-\mathrm{NMR}$ assignments for G4-090s14, $600 \mathrm{MHz}$ in DMSO (2.50 ppm)

\begin{tabular}{|c|c|c|c|c|c|}
\hline Proton & $\mathbf{p p m}^{\mathbf{a}}$ & Number & Type & $\mathbf{J}_{\mathbf{a}}$ & $\mathbf{J}_{\mathbf{b}}$ \\
\hline $\mathrm{H}_{1}$ & 0.891 & 3 & $\mathbf{t}$ & 6.9 & \\
\hline $\mathbf{H}_{2} \& \mathbf{H}_{3}$ & 1.303 & 4 & $\mathbf{m}$ & & \\
\hline $\mathbf{H}_{4}$ & 1.384 & 2 & $\mathbf{m}$ & & \\
\hline $\mathrm{H}_{5}$ & 1.690 & 2 & $\mathbf{m}$ & & \\
\hline $\mathrm{H}_{6}$ & 3.887 & 2 & $\mathbf{m}$ & & \\
\hline $\mathrm{H}_{7}$ & 6.690 & 1 & d & 9.0 & \\
\hline $\mathbf{H}_{8}$ & 6.841 & 1 & dd & 9.0 & 3.0 \\
\hline $\mathrm{H}_{9}$ & 7.224 & 1 & d & 3.0 & \\
\hline $\mathbf{H}_{10}$ & 4.619 & 1 & $\mathbf{t}$ & 8.1 & \\
\hline $\mathbf{H}_{11}$ & 7.183 & 2 & $\mathbf{m}$ & & \\
\hline $\mathrm{H}_{12}$ & 6.975 & 2 & $\mathbf{m}$ & & \\
\hline $\mathrm{H}_{13}$ & 7.239 & 2 & $\mathbf{m}$ & & \\
\hline $\mathrm{H}_{14} \& \mathrm{H}_{15}$ & 7.314 & 4 & $\mathbf{m}$ & & \\
\hline $\mathrm{H}_{16}$ & 3.500 & 2 & $\mathbf{m}$ & & \\
\hline $\mathbf{H}_{17}$ & 7.895 & 3 & b.s. & & \\
\hline
\end{tabular}

a. Taken at the center of the peak.
TABLE A.10: ${ }^{13} \mathrm{C}-\mathrm{NMR}$ assignments for $\mathbf{G 4 -}$ 090s14, $600 \mathrm{MHz}$ in DMSO (39.52 ppm)

\begin{tabular}{|c|c|c|c|c|c|}
\hline Carbon & $\mathbf{p p m}^{\mathbf{a}}$ & Carbon & $\mathbf{p p m}^{\mathbf{a}}$ & Type $^{\text {b }}$ & $\mathbf{J}$ \\
\hline $\mathrm{C}_{1}$ & 13.91 & $\mathrm{C}_{12}$ & 130.33 & & \\
\hline $\mathrm{C}_{2}$ & 22.04 & $\mathrm{C}_{13}$ & 42.08 & & \\
\hline $\mathrm{C}_{3}$ & 31.00 & $\mathrm{C}_{14}$ & 154.04 & d & 2.2 \\
\hline $\mathrm{C}_{4}$ & 25.21 & $\mathrm{C}_{15}$ & 118.92 & d & 9.1 \\
\hline $\mathrm{C}_{5}$ & 28.74 & $\mathrm{C}_{16}$ & 116.29 & d & 24.1 \\
\hline $\mathrm{C}_{6}$ & 68.07 & $\mathrm{C}_{17}$ & 157.61 & d & 238.4 \\
\hline $\mathrm{C}_{7}$ & 149.56 & $\mathrm{C}_{18}$ & 140.32 & & \\
\hline $\mathrm{C}_{8}$ & 113.12 & $\mathrm{C}_{19}$ & 128.03 & & \\
\hline $\mathrm{C}_{9}$ & 118.38 & $\mathrm{C}_{20}$ & 128.48 & & \\
\hline $\mathrm{C}_{10}$ & 152.46 & $\mathrm{C}_{21}$ & 126.90 & & \\
\hline $\mathrm{C}_{11}$ & 119.36 & $\mathrm{C}_{22}$ & 41.63 & & \\
\hline
\end{tabular}

a. Taken at the center of the peak.

b. Singlet unless noted otherwise. 
ET-92: 2-(5-(4-fluorophenoxy)-2-(hexyloxy)phenyl)-2-phenylethanamine hydrochloFIGURE A.33: ${ }^{1} \mathrm{H}-\mathrm{NMR}$ of ET-92 (G4-090s14).

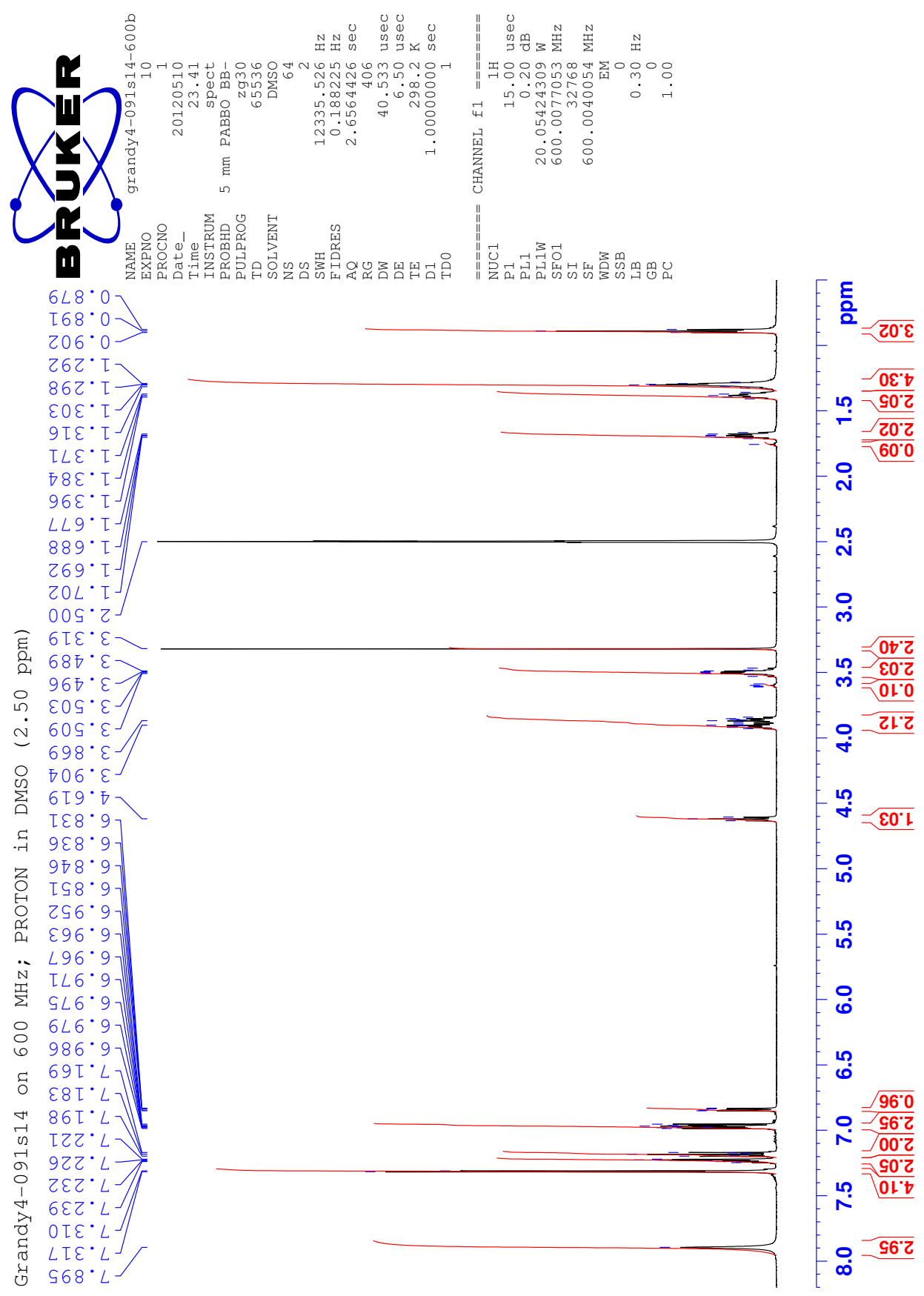


ET-92: 2-(5-(4-fluorophenoxy)-2-(hexyloxy)phenyl)-2-phenylethanamine hydrochloFIGURE A.34: COSY-NMR of ET-92 (G4-090s14).

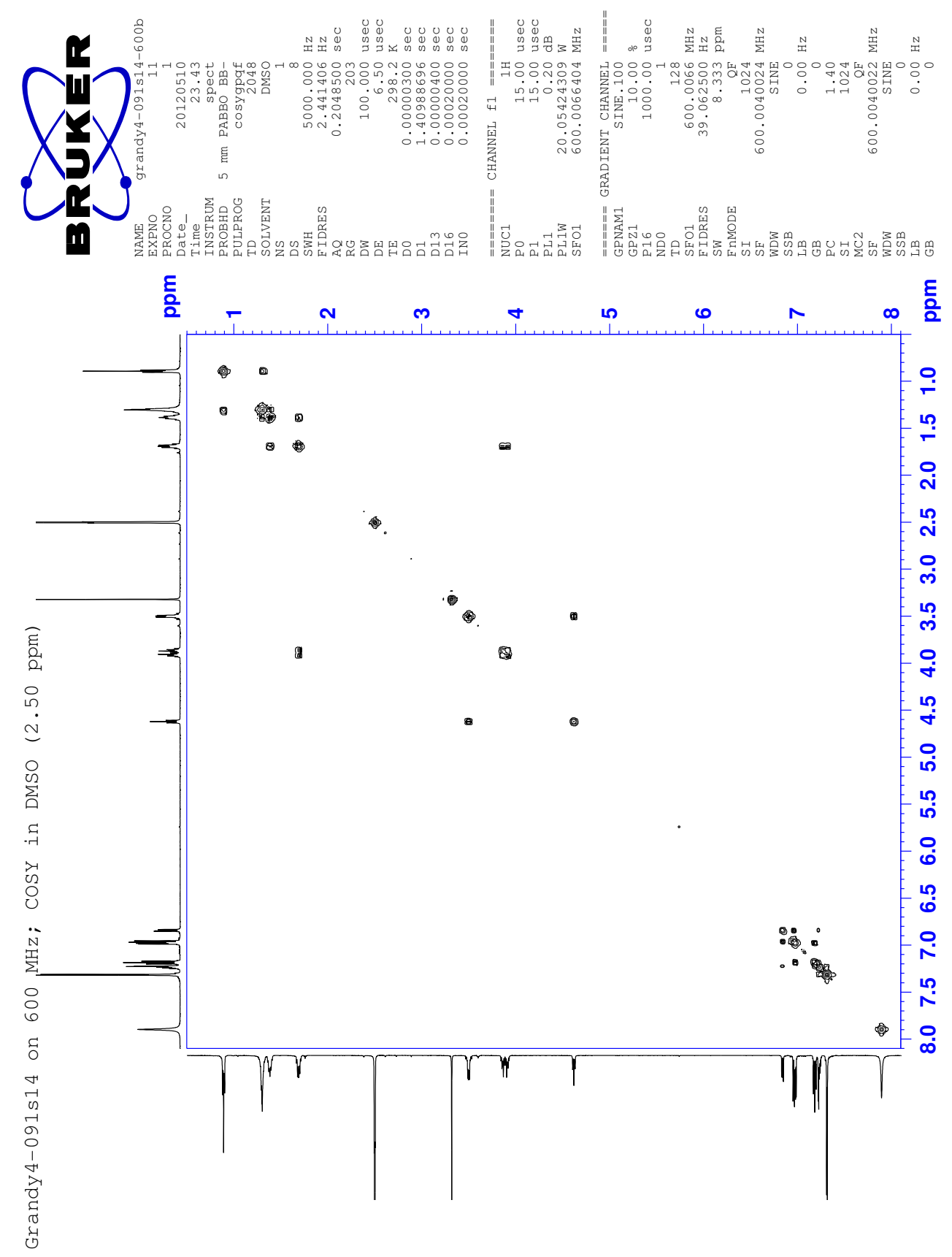


ET-92: 2-(5-(4-fluorophenoxy)-2-(hexyloxy)phenyl)-2-phenylethanamine hydrochloFIGURE A.35: ${ }^{13}$ C-NMR of ET-92 (G4-090s14).

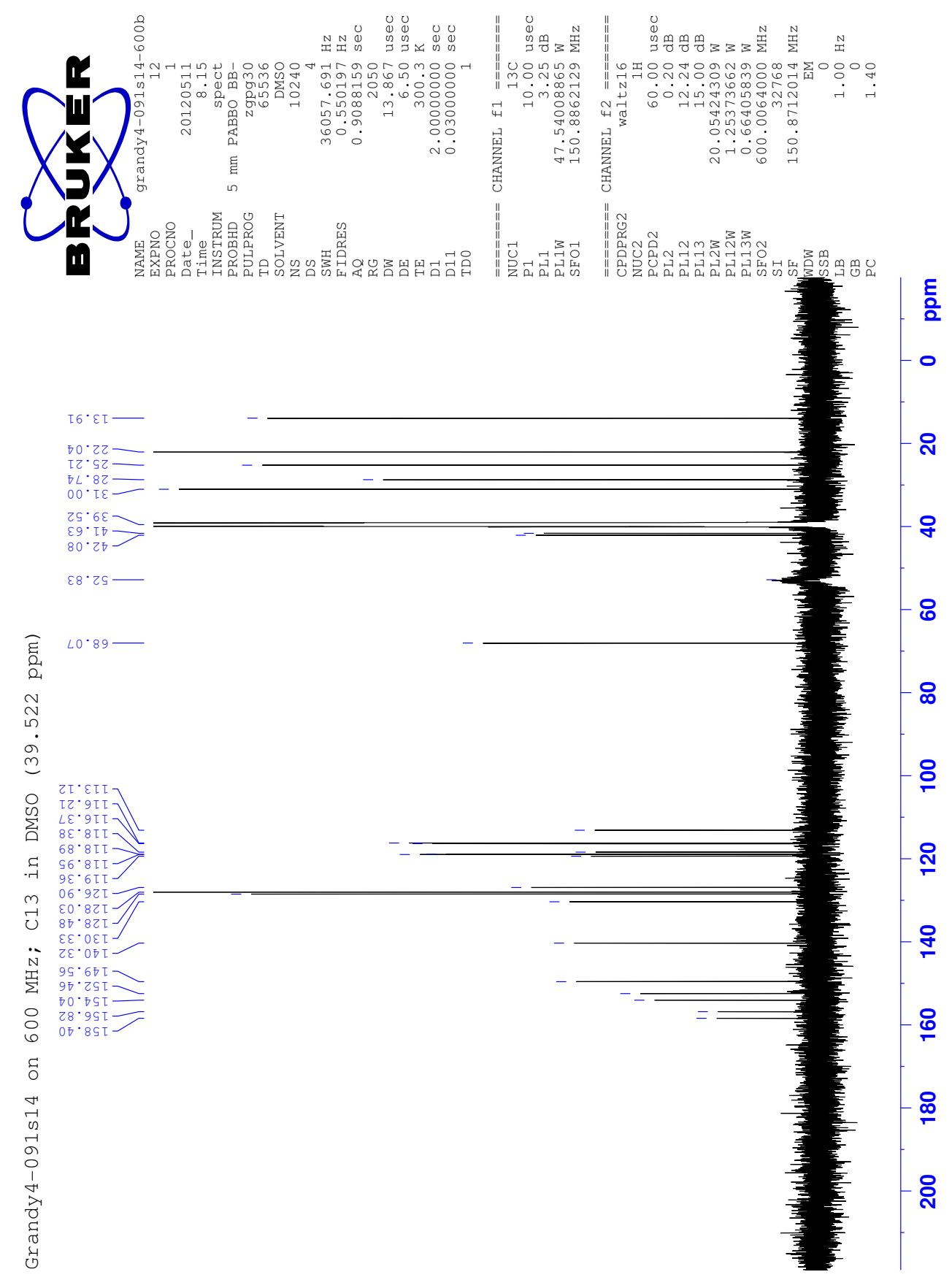


ET-92: 2-(5-(4-fluorophenoxy)-2-(hexyloxy)phenyl)-2-phenylethanamine hydrochloFIGURE A.36: HSQC-NMR of ET-92 (G4-090s14).

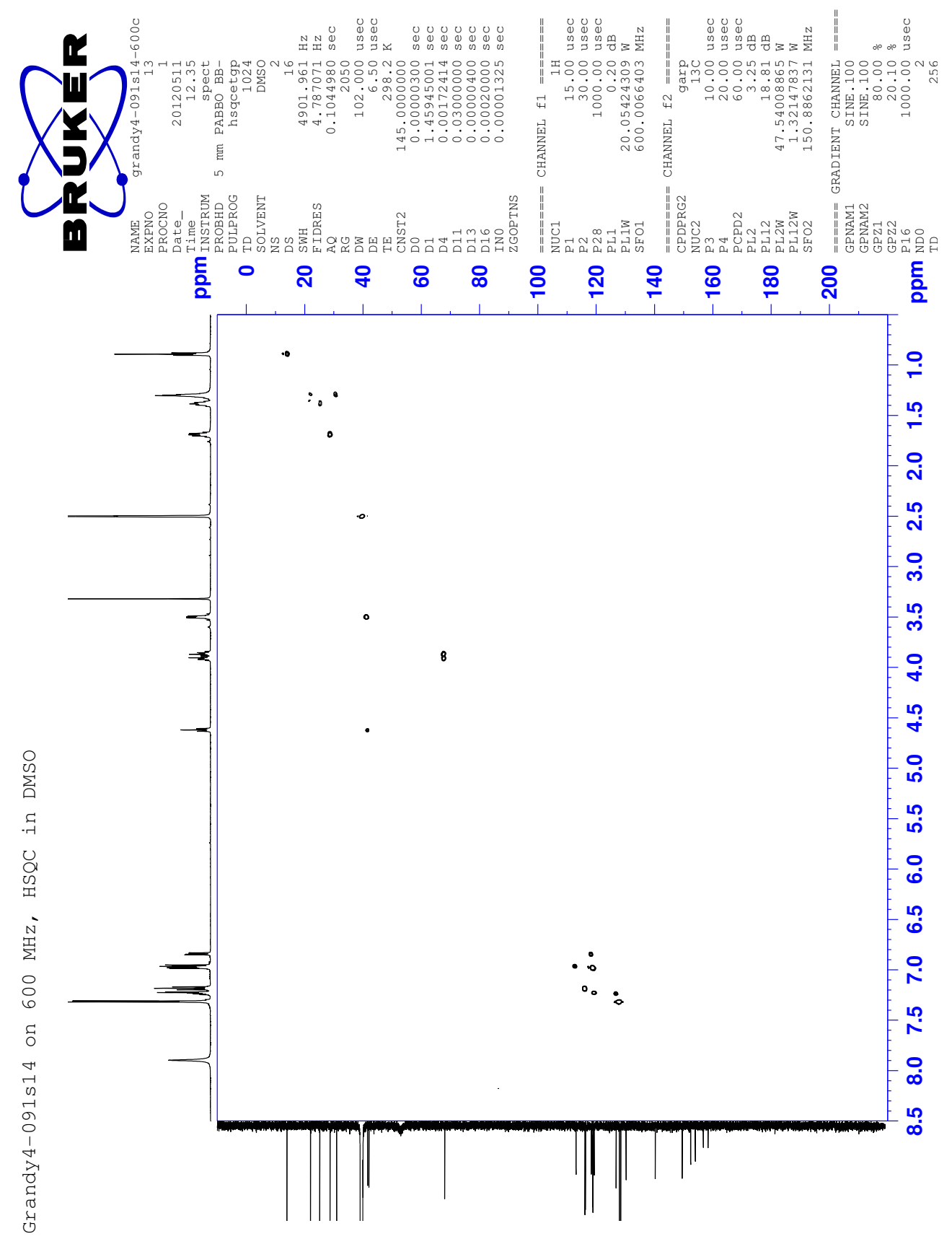


ET-92: 2-(5-(4-fluorophenoxy)-2-(hexyloxy)phenyl)-2-phenylethanamine hydrochloFIGURE A.37: HMBC-NMR of ET-92 (G4-090s14).

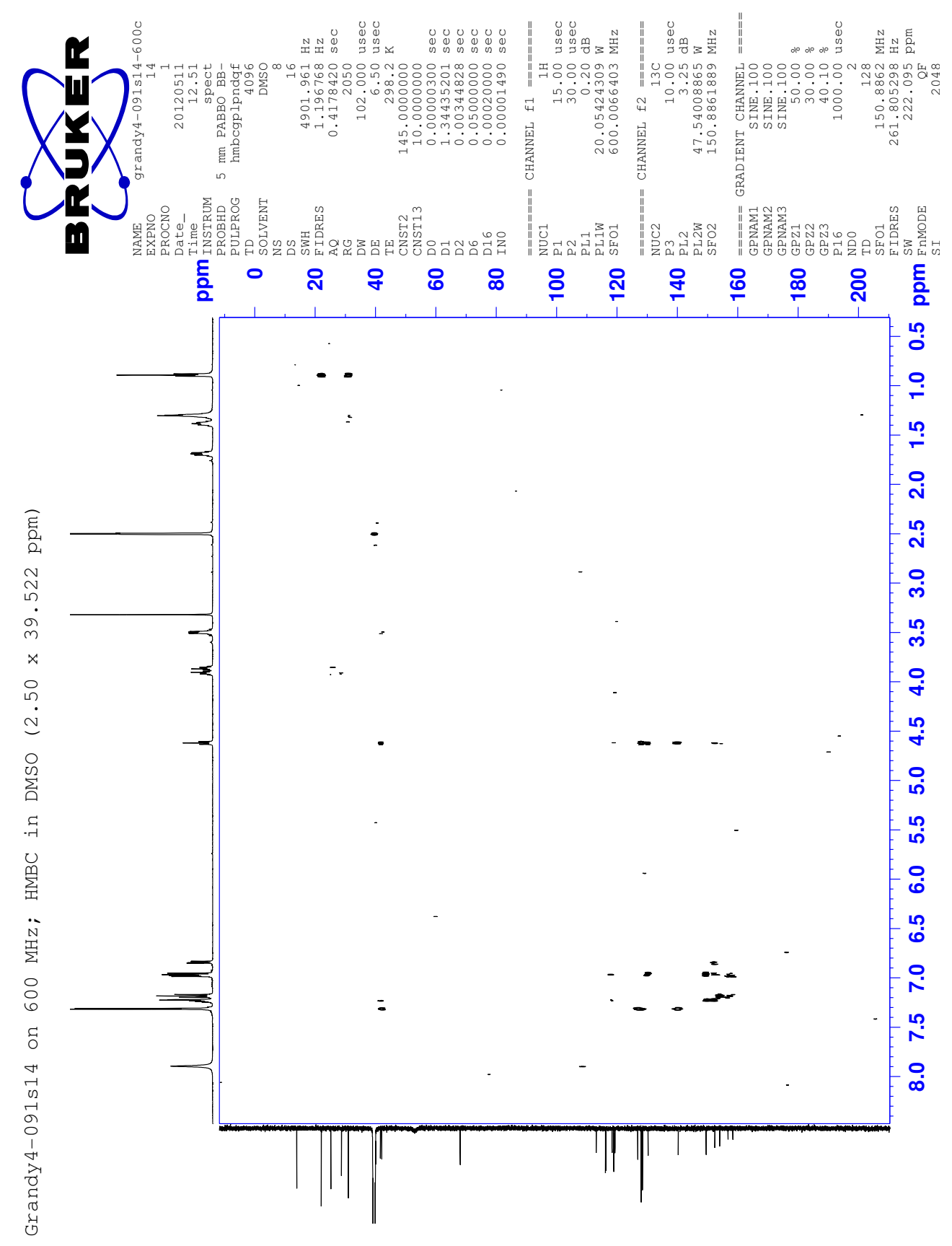


ET-92: 2-(5-(4-fluorophenoxy)-2-(hexyloxy)phenyl)-2-phenylethanamine hydrochloFIGURE A.38: NOESY-NMR of ET-92 (G4-090s14).

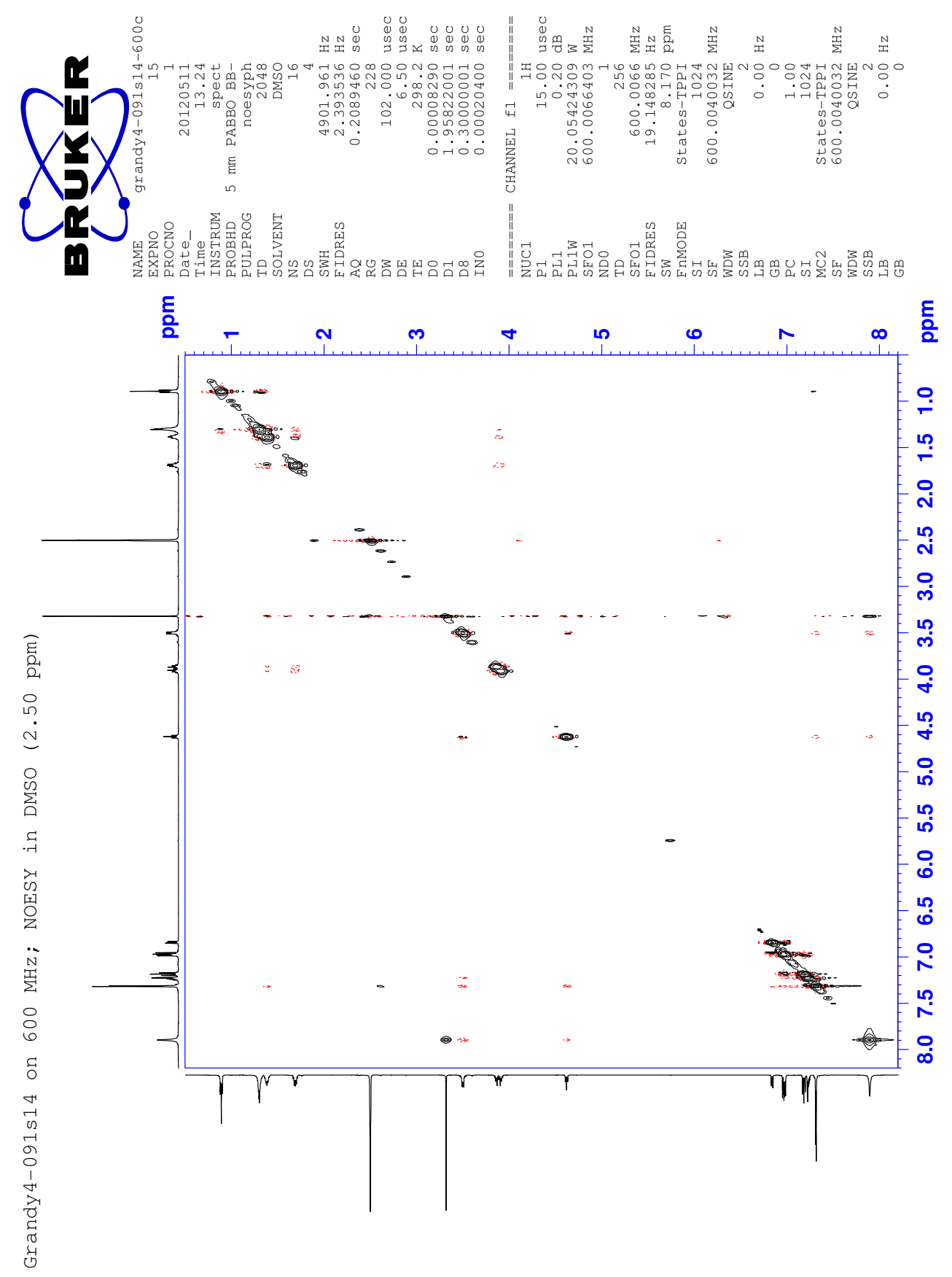


ET-92: 2-(5-(4-fluorophenoxy)-2-(hexyloxy)phenyl)-2-phenylethanamine hydrochloFIGURE A.39: ${ }^{19}$ F-NMR of ET-92 (G4-090s14), $400 \mathrm{MHz}$ with $\mathrm{C}_{6} \mathrm{~F}_{6}(-164.0 \mathrm{ppm})$.

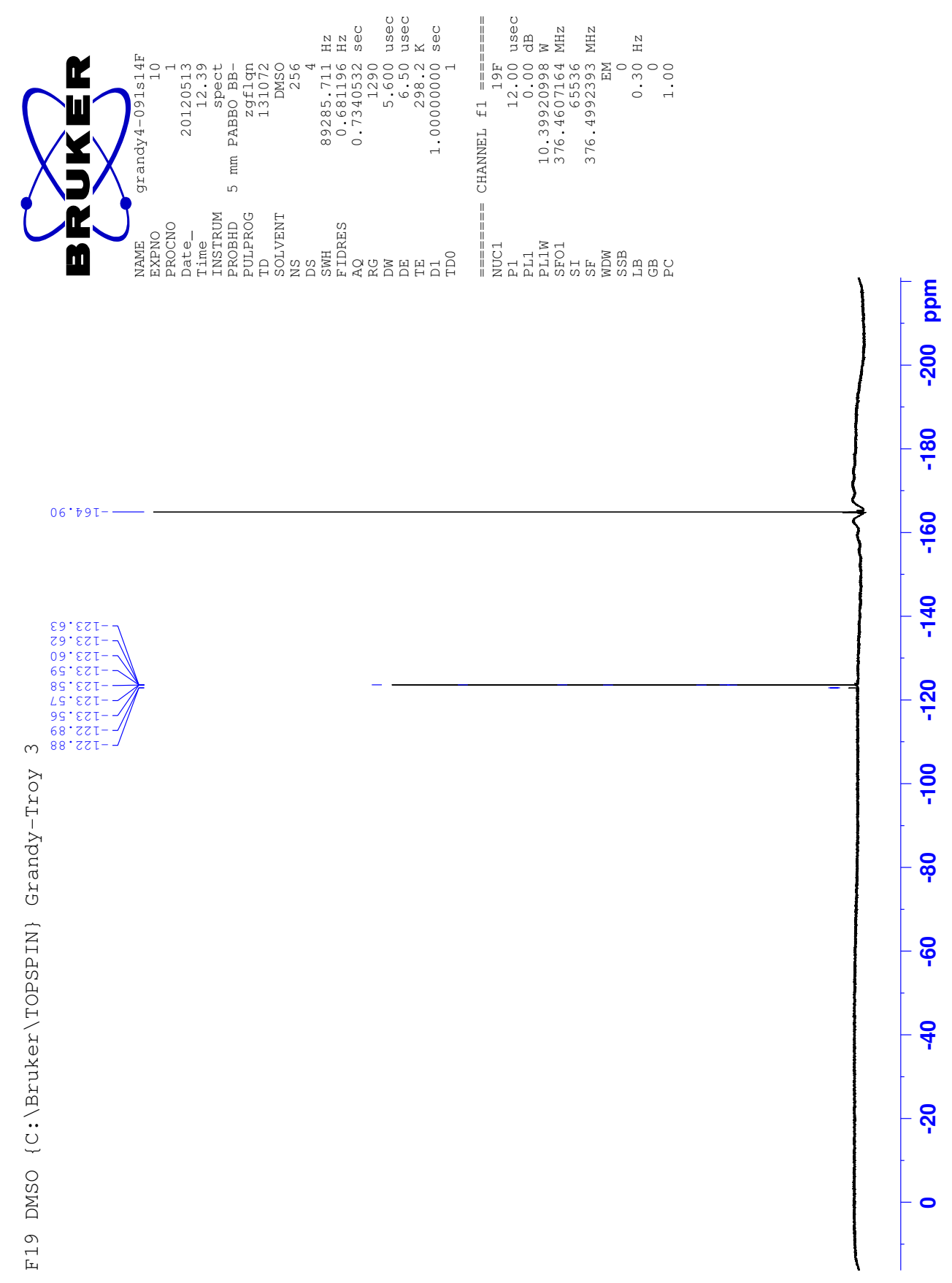


(2-hydroxy-5-nitrophenyl)(pyrrolidin-1-yl)methone: G5-088s8

\section{A.6 (2-hydroxy-5-nitrophenyl)(pyrrolidin-1-yl)methone: G5-088s8}

FIGURE A.40: Structure of G5-088s8.
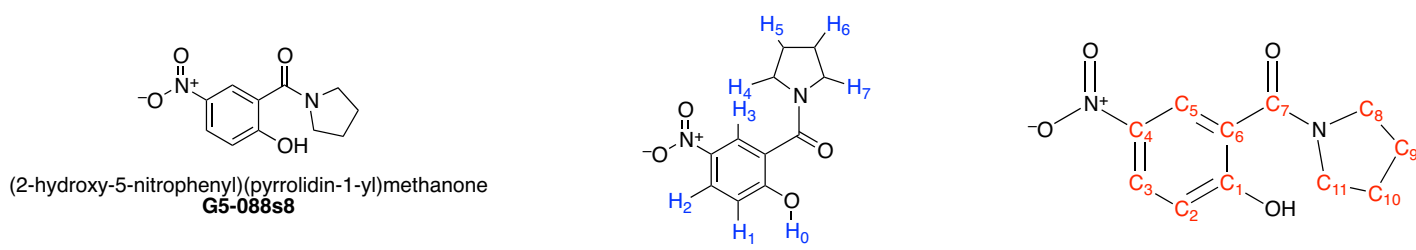

TABLE A.11: ${ }^{1} \mathrm{H}-\mathrm{NMR}$ assignments for G5-088s8, $400 \mathrm{MHz}$ in DMSO (2.50 ppm)

\begin{tabular}{|c|c|c|c|c|c|}
\hline Proton & $\mathbf{p p m}^{\mathbf{a}}$ & Number & Type & $\mathbf{J}_{\mathbf{a}}$ & $\mathbf{J}_{\mathbf{b}}$ \\
\hline $\mathbf{H}_{\mathbf{0}}$ & 11.56 & 1 & $\mathbf{s}$ & & \\
\hline $\mathbf{H}_{1}$ & 7.07 & 1 & d & 9.07 & \\
\hline $\mathbf{H}_{2}$ & 8.16 & 1 & dd & 9.06 & 2.91 \\
\hline $\mathbf{H}_{3}$ & 8.06 & 1 & d & 2.88 & \\
\hline $\mathbf{H}_{4}$ & 3.23 & 2 & $\mathbf{t}$ & 6.74 & \\
\hline $\mathrm{H}_{5} \& \mathrm{H}_{6}$ & 1.84 & 4 & $\mathbf{m}$ & & \\
\hline $\mathrm{H}_{7}$ & 3.46 & 2 & $\mathbf{t}$ & 6.74 & \\
\hline
\end{tabular}

a. Taken at the center of the peak.

TABLE A.12: ${ }^{13} \mathrm{C}-\mathrm{NMR}$ assignments for G5-088s8, $400 \mathrm{MHz}$ in DMSO (39.52 ppm)

\begin{tabular}{|c|c|}
\hline Carbon & $p p m$ \\
\hline$C_{1}$ & 159.74 \\
\hline$C_{2}$ & 116.50 \\
\hline$C_{3}$ & 126.26 \\
\hline$C_{4}$ & 139.33 \\
\hline$C_{5}$ & 125.91 \\
\hline$C_{6}$ & 124.29 \\
\hline$C_{7}$ & 164.47 \\
\hline$C_{8}$ & 45.43 \\
\hline$C_{9}$ & 23.94 \\
\hline$C_{10}$ & 25.38 \\
\hline$C_{11}$ & 46.95 \\
\hline
\end{tabular}


(2-hydroxy-5-nitrophenyl)(pyrrolidin-1-yl)methone: G5-088s8

FIGURE A.41: ${ }^{1} \mathrm{H}-\mathrm{NMR}$ of G5-088s8.

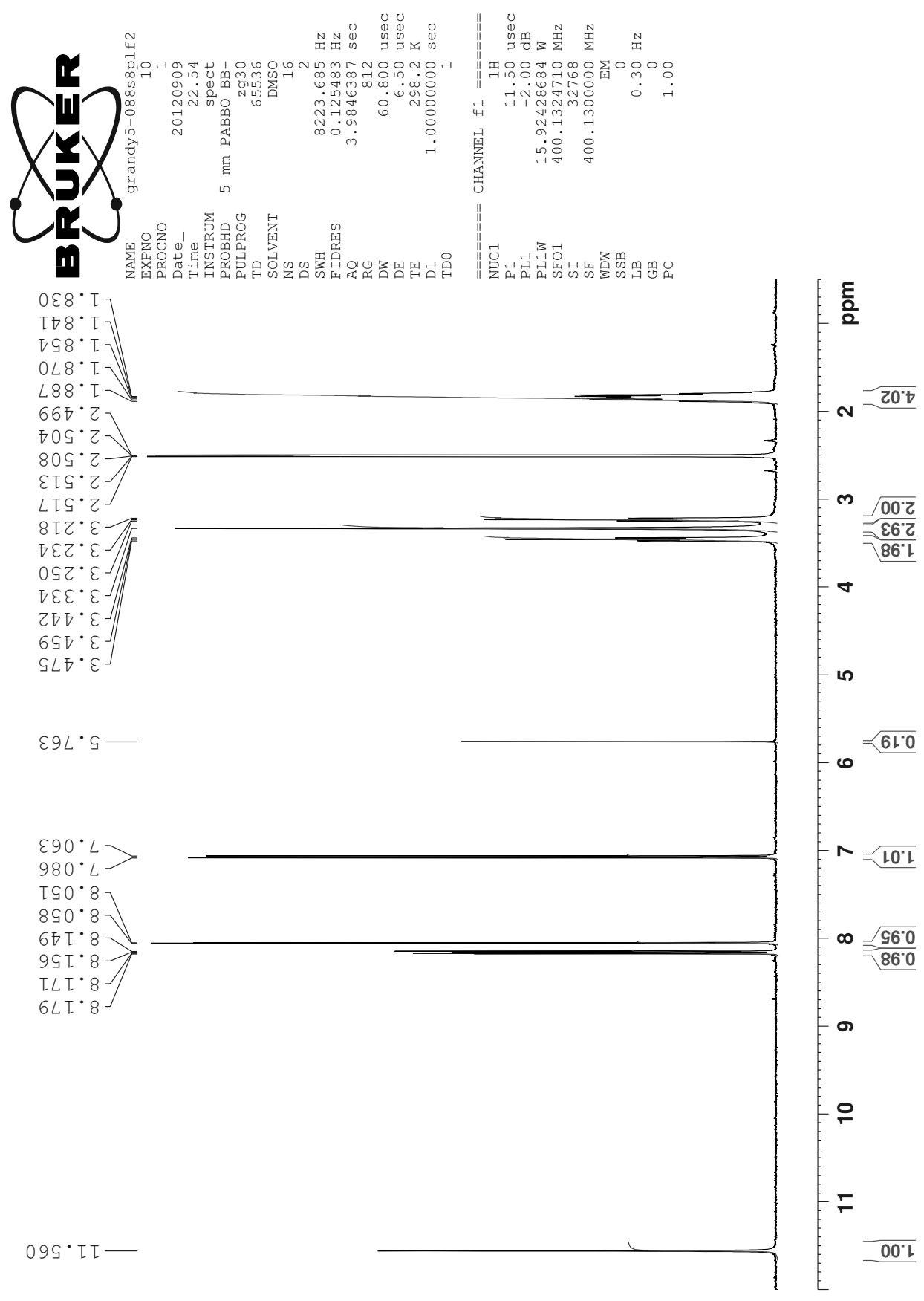


(2-hydroxy-5-nitrophenyl)(pyrrolidin-1-yl)methone: G5-088s8

FIGURE A.42: COSY-NMR of G5-088s8.

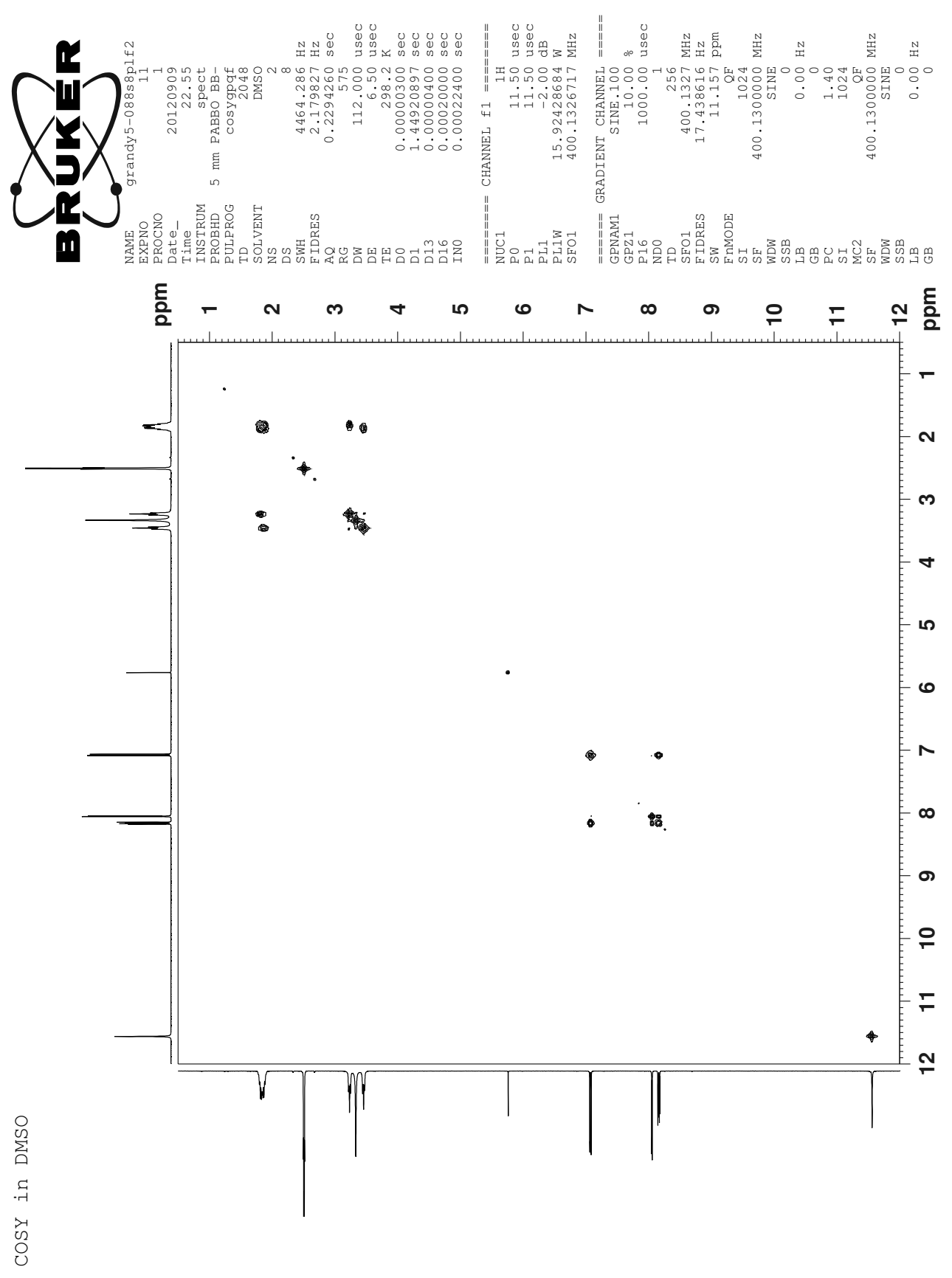


(2-hydroxy-5-nitrophenyl)(pyrrolidin-1-yl)methone: G5-088s8

FIGURE A.43: ${ }^{13} \mathrm{C}-\mathrm{NMR}$ of G5-088s8.

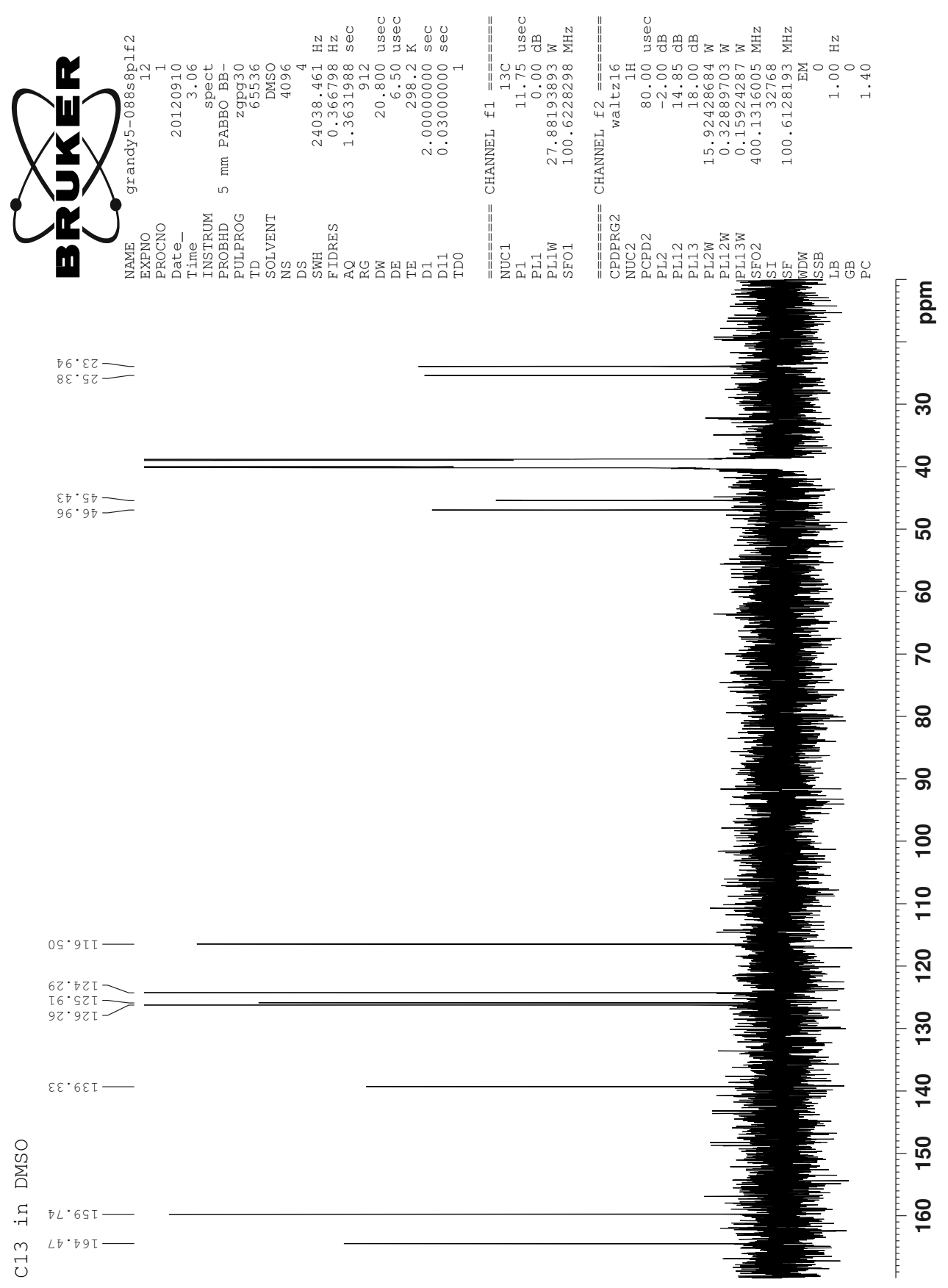


(2-hydroxy-5-nitrophenyl)(pyrrolidin-1-yl)methone: G5-088s8

FIGURE A.44: HSQC-NMR of G5-088s8.

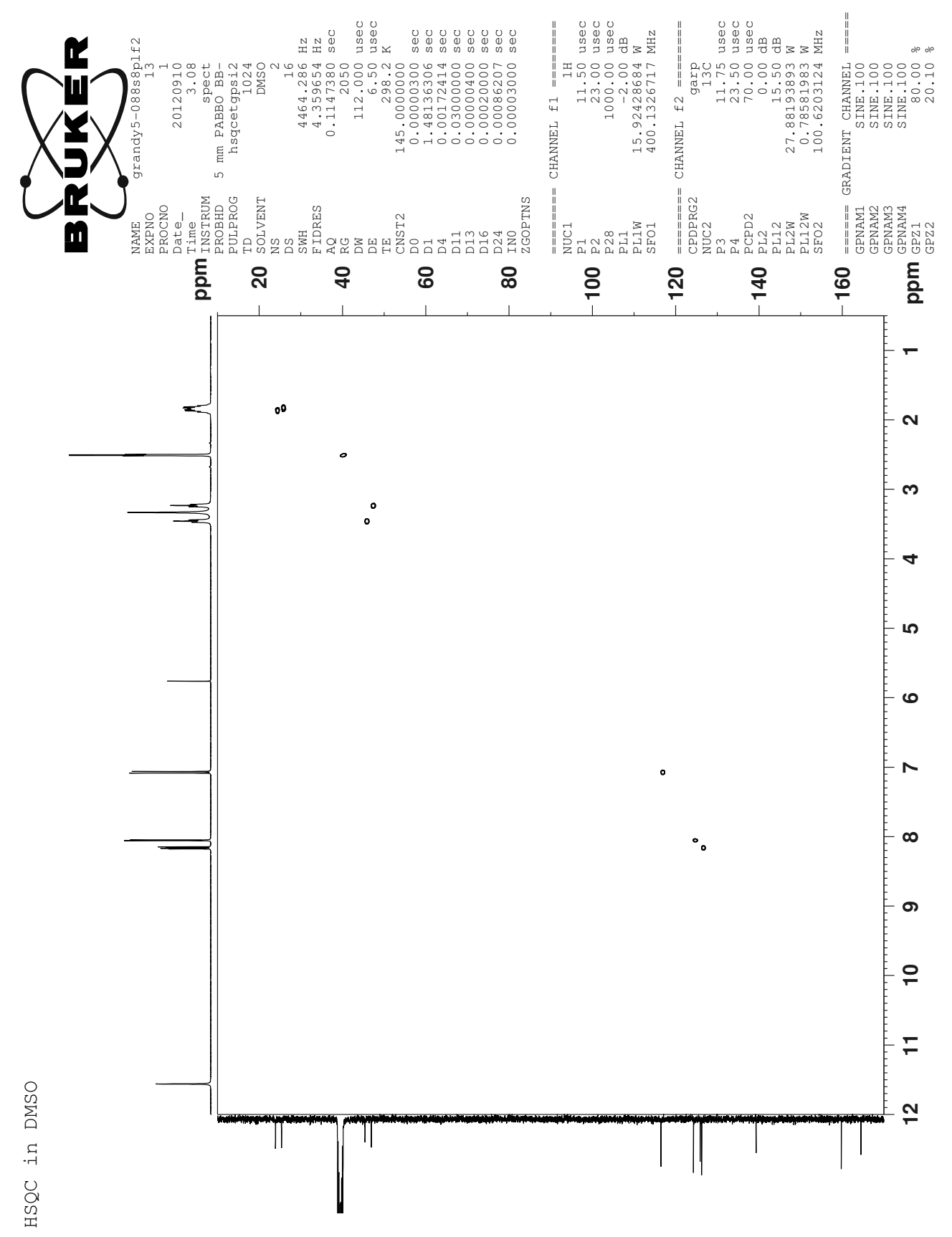


(2-hydroxy-5-nitrophenyl)(pyrrolidin-1-yl)methone: G5-088s8

FIGURE A.45: HMBC-NMR of G5-088s8.

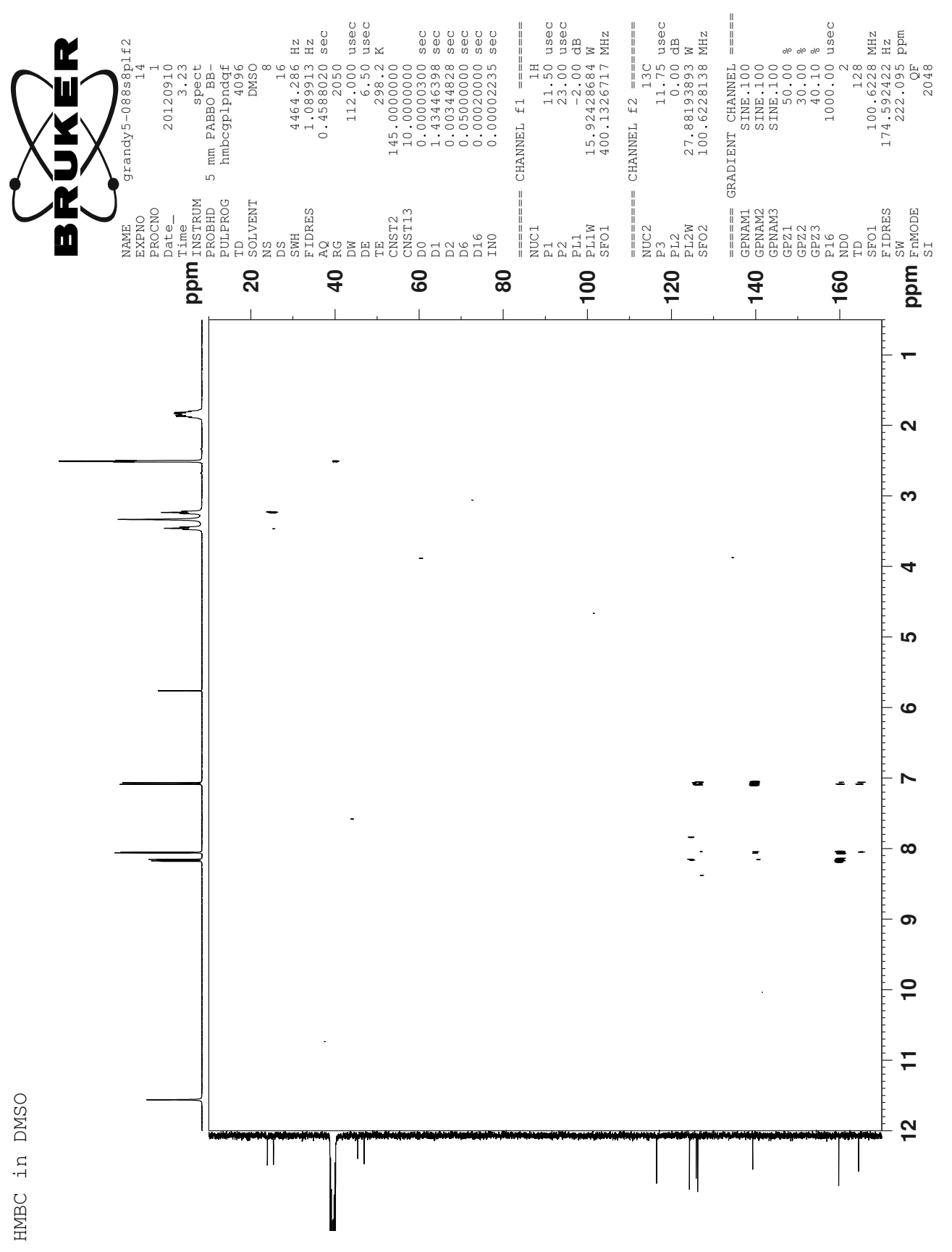


(2-hydroxy-5-nitrophenyl)(pyrrolidin-1-yl)methone: G5-088s8

FIGURE A.46: NOESY-NMR of G5-088s8.
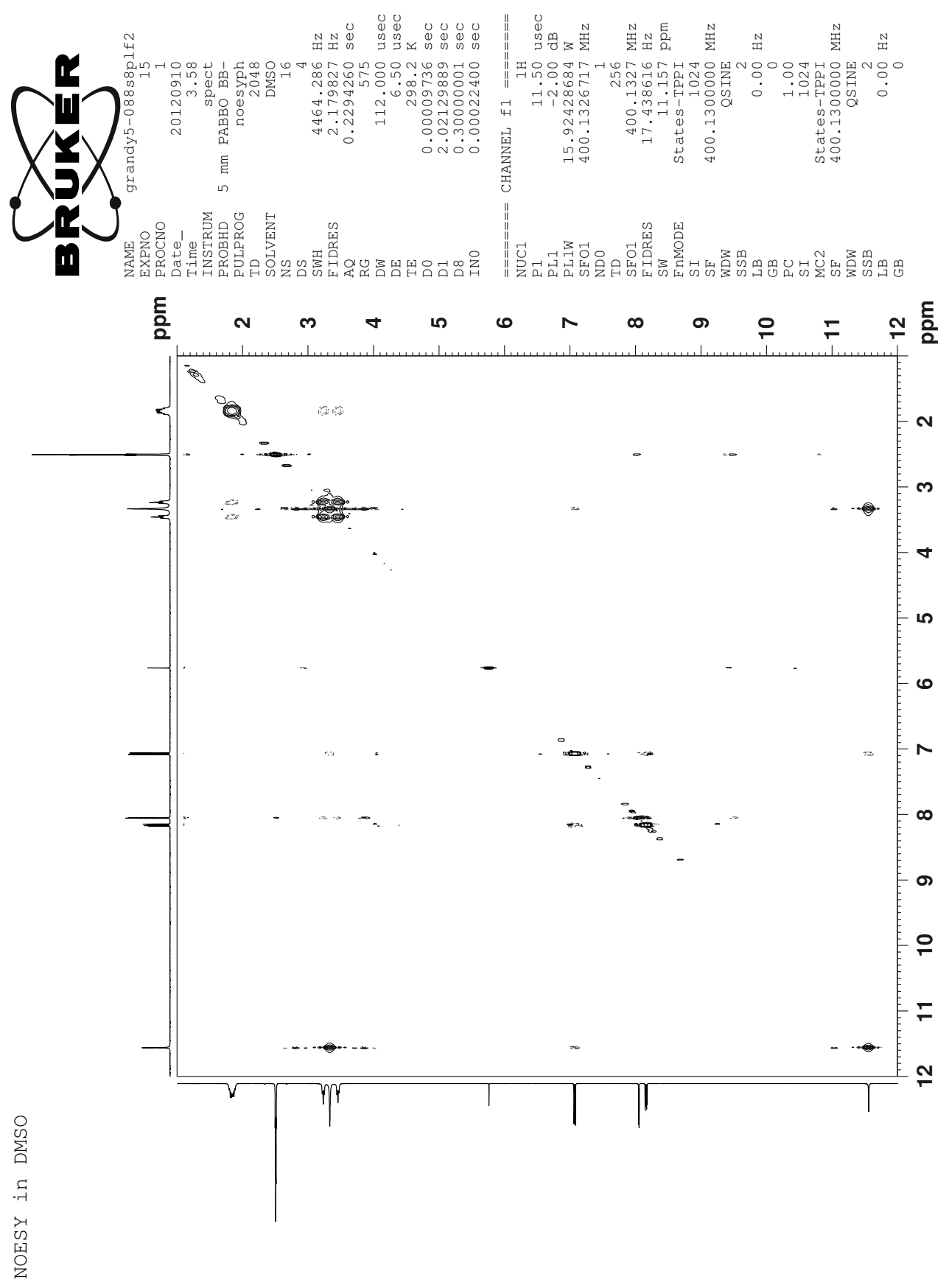
(2-(hexyloxy)-5-nitrophenyl)(pyrrolidin-1-yl)methone: G5-090s10

\section{A.7 (2-(hexyloxy)-5-nitrophenyl)(pyrrolidin-1-yl)methone: G5-090s10}

FIGURE A.47: Structure of G5-090s10.

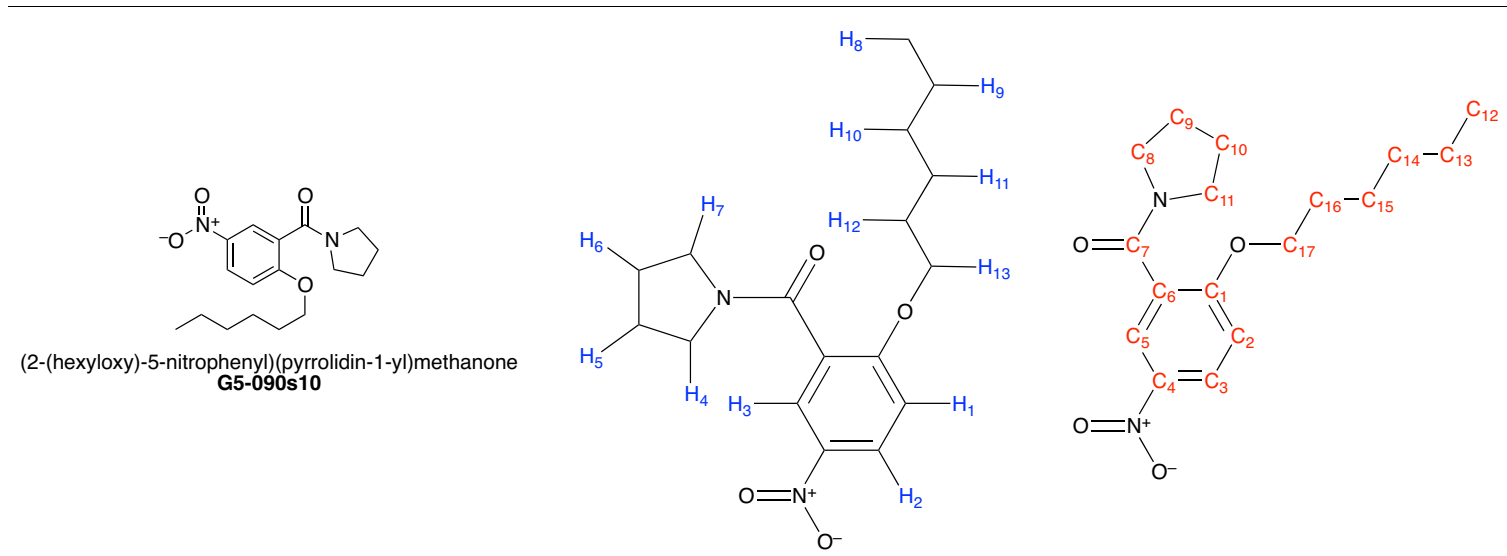

TABLE A.13: ${ }^{1} \mathrm{H}-\mathrm{NMR}$ assignments for $\mathbf{G 5}-$ 090s10, $400 \mathrm{MHz}$ in DMSO (2.50 ppm)

\begin{tabular}{|c|c|c|c|c|c|}
\hline Proton & ppm $^{\mathrm{a}}$ & Number & Type & $\mathrm{J}_{\mathbf{a}}$ & $\mathrm{J}_{\mathbf{b}}$ \\
\hline $\mathrm{H}_{1}$ & 7.31 & 1 & $\mathbf{d}$ & 9.24 & \\
\hline $\mathrm{H}_{2}$ & 8.28 & 1 & dd & 9.19 & 2.90 \\
\hline $\mathrm{H}_{3}$ & 8.06 & 1 & $\mathrm{~d}$ & 2.89 & \\
\hline $\mathrm{H}_{4}$ & 3.13 & 2 & $\mathrm{t}$ & 6.55 & \\
\hline $\mathrm{H}_{5} \& \mathrm{H}_{6}$ & 1.84 & 4 & $\mathrm{~m}$ & & \\
\hline $\mathrm{H}_{7}$ & 3.46 & 2 & $\mathrm{t}$ & 6.55 & \\
\hline $\mathrm{H}_{8}$ & $\mathbf{0 . 8 8}$ & 3 & $\mathrm{t}$ & 6.83 & \\
\hline $\mathrm{H}_{9} \& \mathrm{H}_{10}$ & 1.30 & 4 & $\mathrm{~m}$ & & \\
\hline $\mathrm{H}_{11}$ & 1.39 & 2 & $\mathrm{~m}$ & & \\
\hline $\mathrm{H}_{12}$ & 1.71 & 2 & $\mathrm{~m}$ & & \\
\hline $\mathrm{H}_{13}$ & 4.18 & 2 & $\mathrm{t}$ & 6.27 & \\
\hline
\end{tabular}

a. Taken at the center of the peak.
TABLE A.14: ${ }^{13} \mathrm{C}-\mathrm{NMR}$ assignments for G5090s10, $400 \mathrm{MHz}$ in DMSO (39.52 ppm)

\begin{tabular}{|c|c|c|c|}
\hline Carbon & $p p m$ & Carbon & $p p m$ \\
\hline$C_{1}$ & 159.38 & $C_{10}$ & 25.36 \\
\hline$C_{2}$ & 112.81 & $C_{11}$ & 46.85 \\
\hline$C_{3}$ & 126.30 & $C_{12}$ & 13.79 \\
\hline$C_{4}$ & 140.42 & $C_{13}$ & 22.02 \\
\hline$C_{5}$ & 123.28 & $C_{14}$ & 30.77 \\
\hline$C_{6}$ & 128.11 & $C_{15}$ & 24.93 \\
\hline$C_{7}$ & 163.89 & $C_{16}$ & 28.25 \\
\hline$C_{8}$ & 45.28 & $C_{17}$ & 68.99 \\
\hline$C_{9}$ & 24.06 & - & - \\
\hline
\end{tabular}


(2-(hexyloxy)-5-nitrophenyl)(pyrrolidin-1-yl)methone: G5-090s10

FIGURE A.48: ${ }^{1} \mathrm{H}-\mathrm{NMR}$ of G5-090s10.

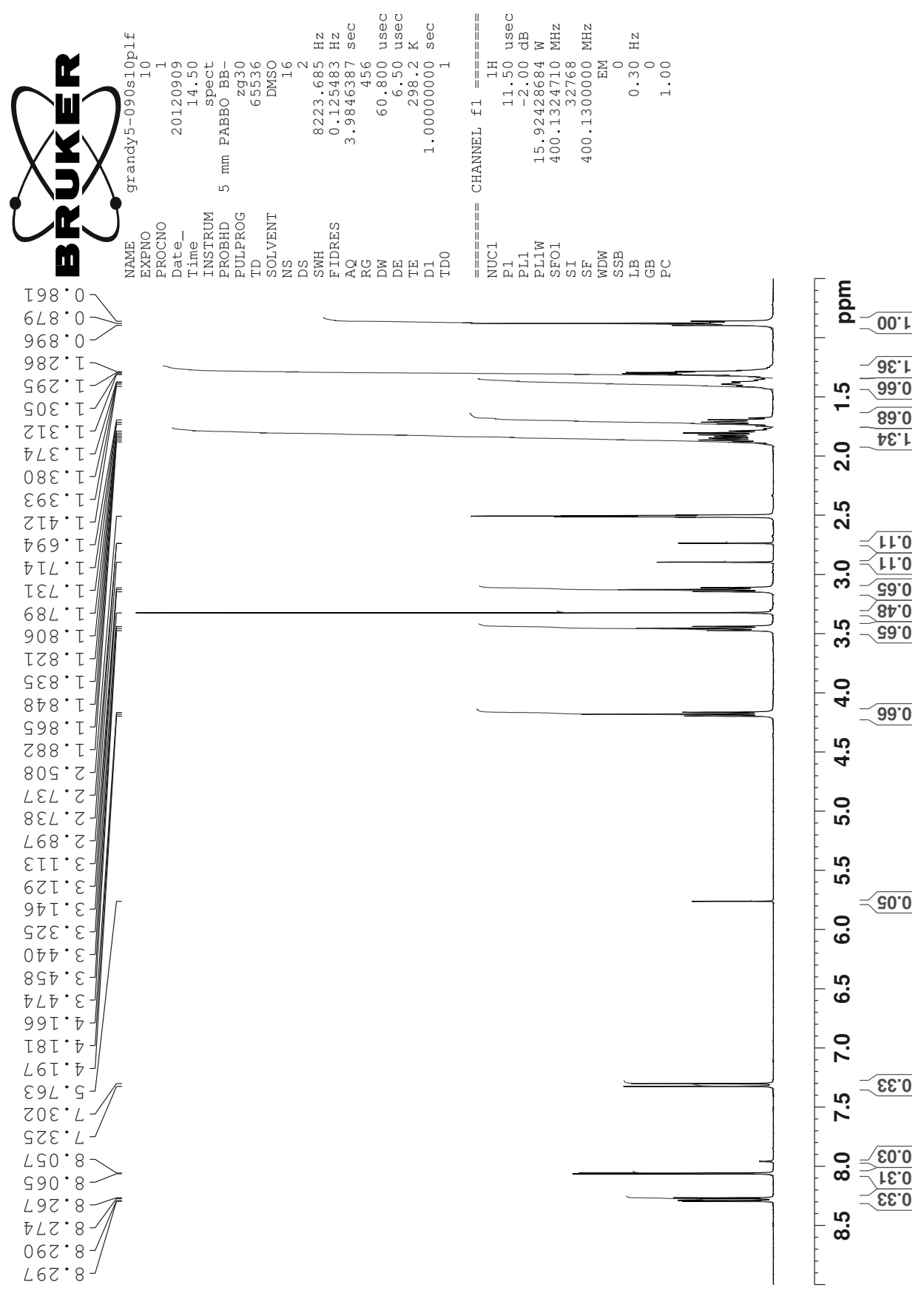


(2-(hexyloxy)-5-nitrophenyl)(pyrrolidin-1-yl)methone: G5-090s10

FIGURE A.49: COSY-NMR of G5-090s10.

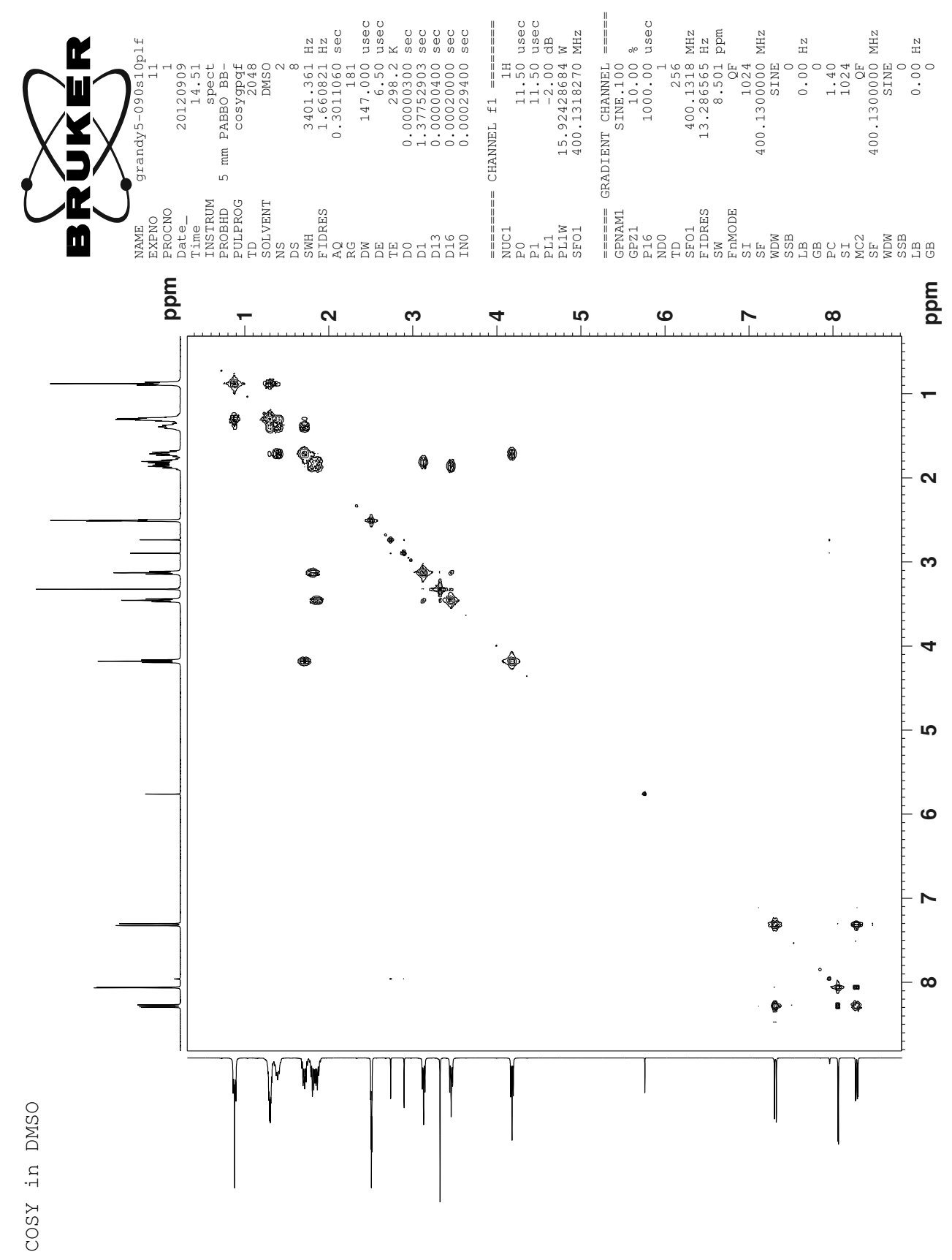


(2-(hexyloxy)-5-nitrophenyl)(pyrrolidin-1-yl)methone: G5-090s10

FIGURE A.50: ${ }^{13} \mathrm{C}-\mathrm{NMR}$ of G5-090s10.

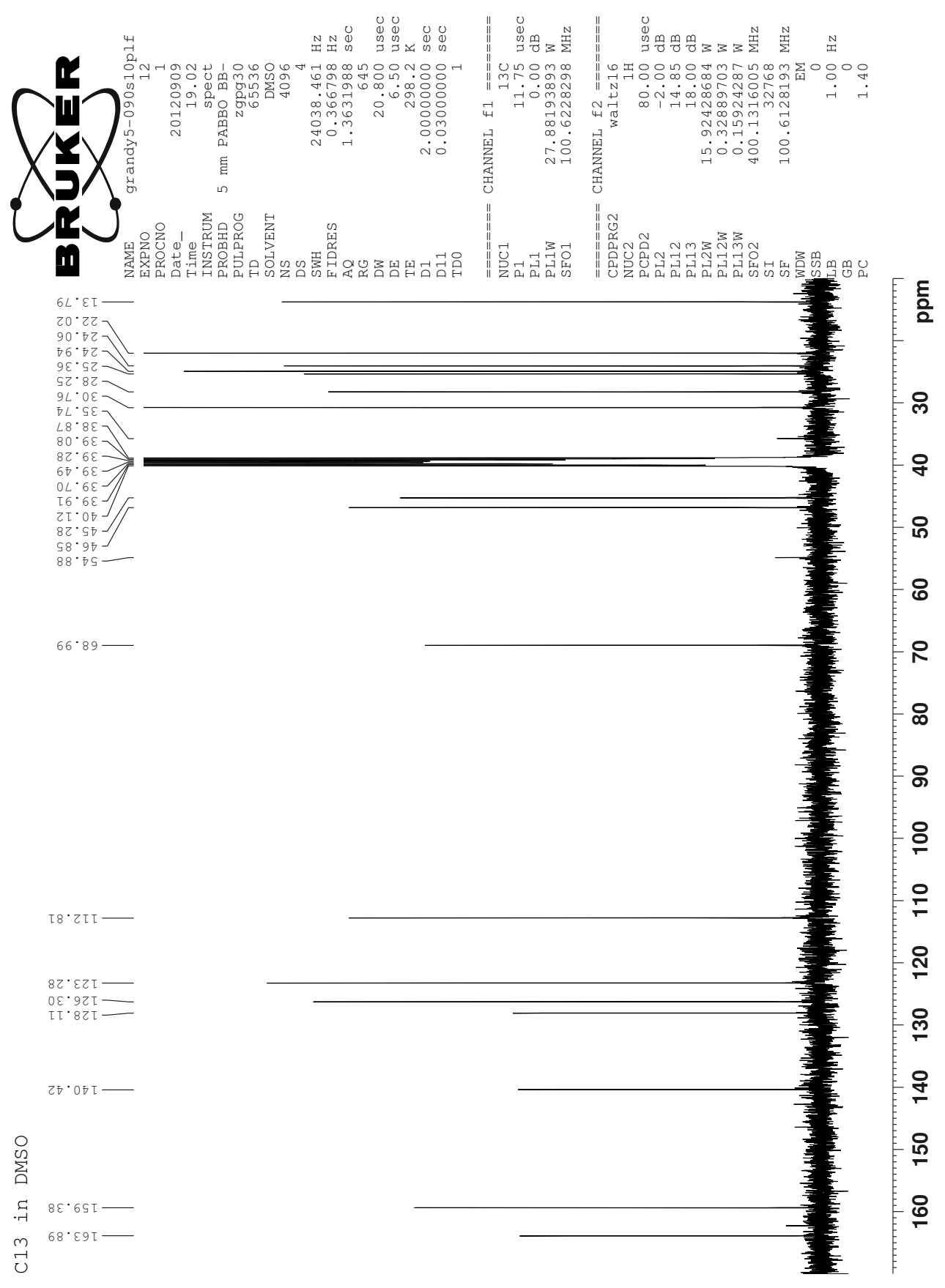


(2-(hexyloxy)-5-nitrophenyl)(pyrrolidin-1-yl)methone: G5-090s10

FIGURE A.51: HSQC-NMR of G5-090s10.

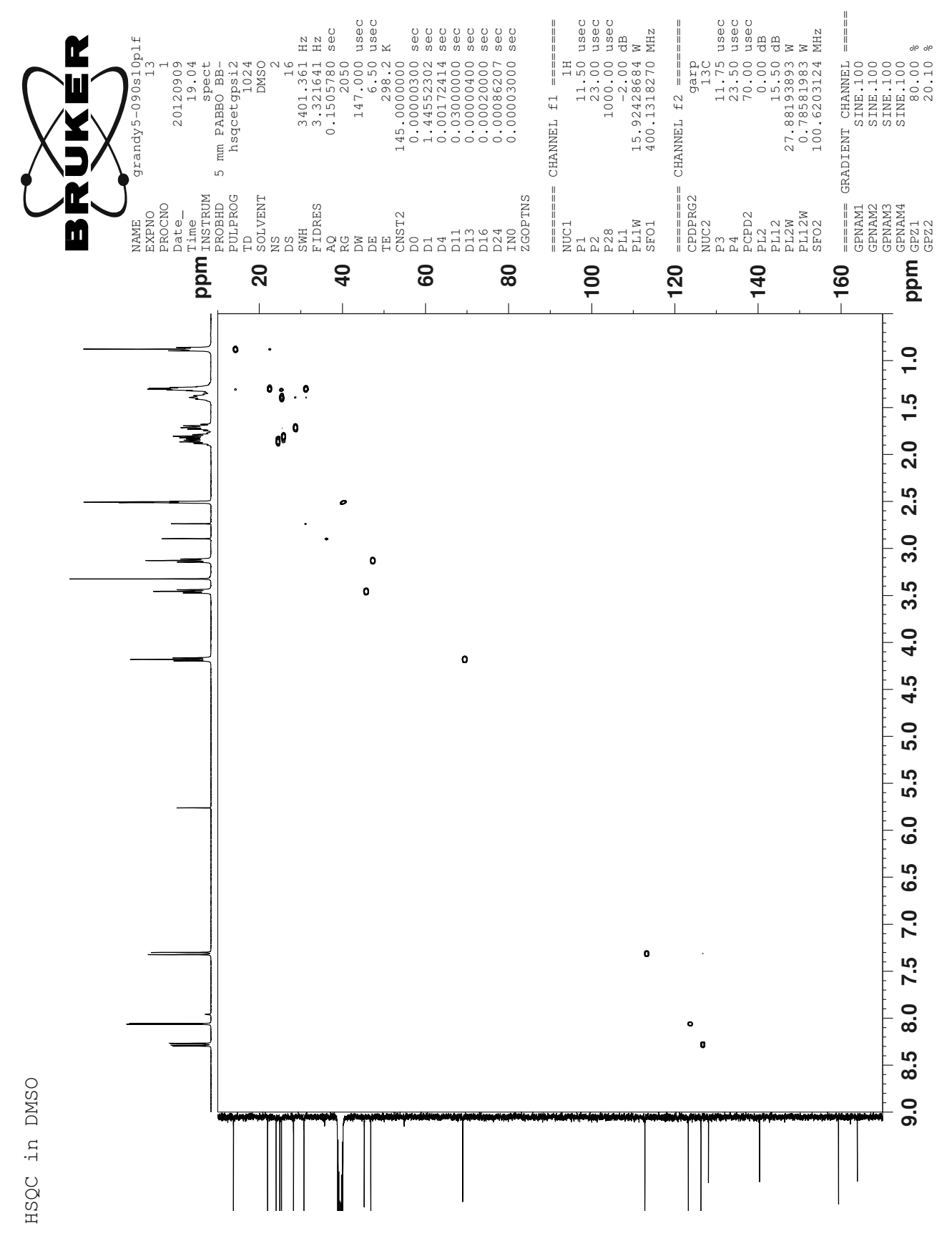


(2-(hexyloxy)-5-nitrophenyl)(pyrrolidin-1-yl)methone: G5-090s10

FIGURE A.52: HMBC-NMR of G5-090s10.

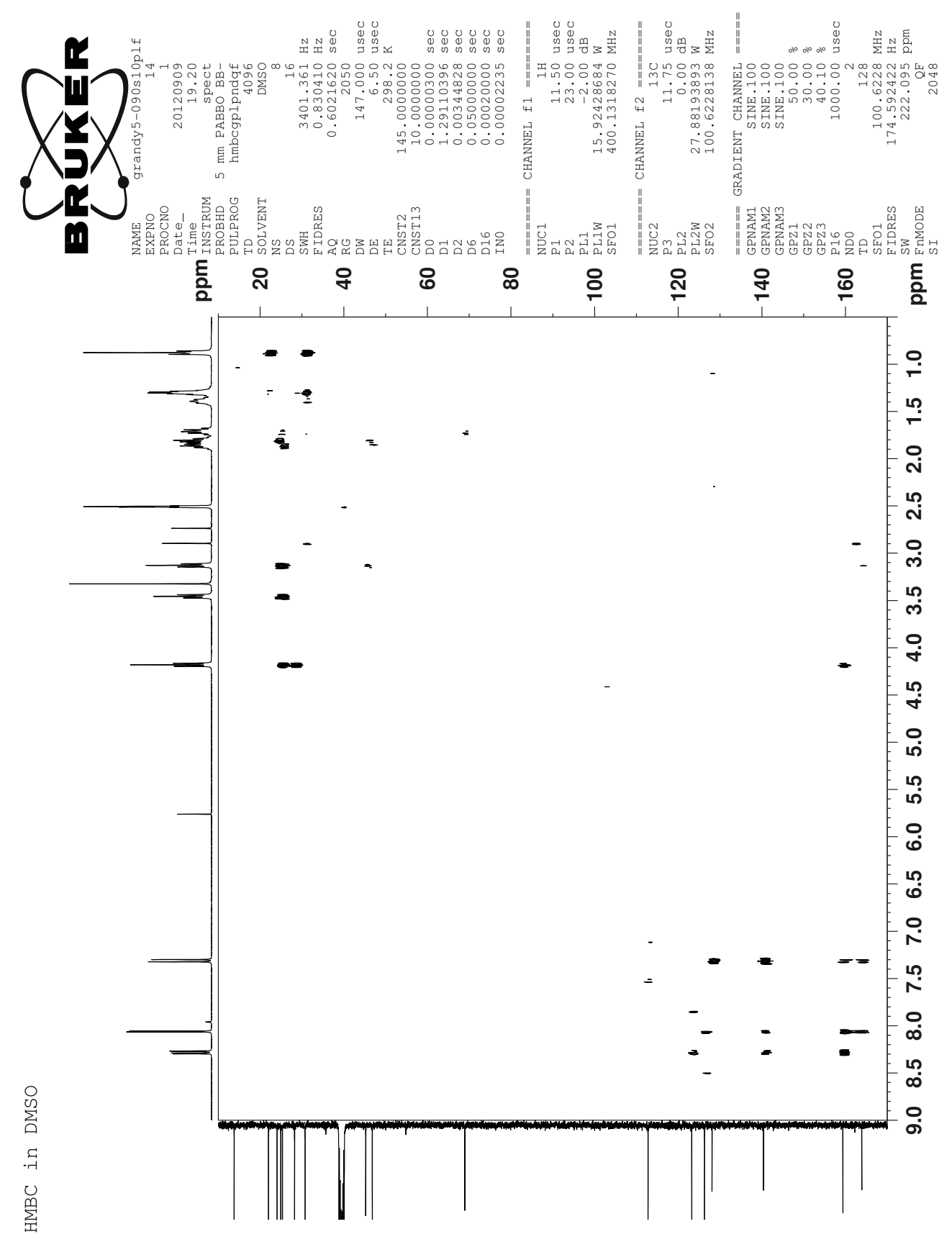


(2-(hexyloxy)-5-nitrophenyl)(pyrrolidin-1-yl)methone: G5-090s10

FIGURE A.53: NOESY-NMR of G5-090s10.
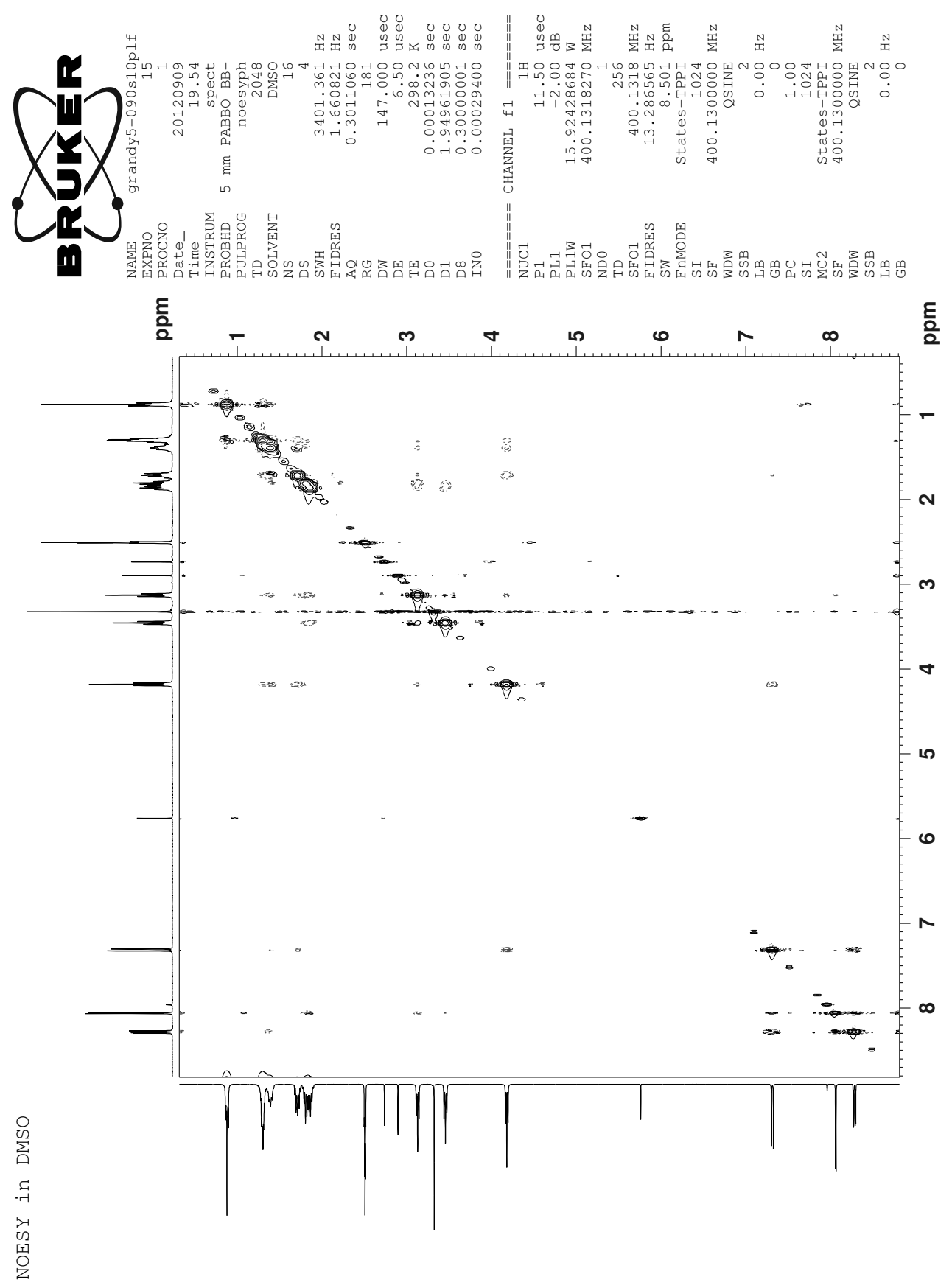
(5-amino-2-(hexyloxy)phenyl)(pyrrolidin-1-yl)methone: G5-106s7

\section{A.8 (5-amino-2-(hexyloxy)phenyl)(pyrrolidin-1-yl)methone: G5-106s 7}

FIGURE A.54: Structure of G5-106s7.
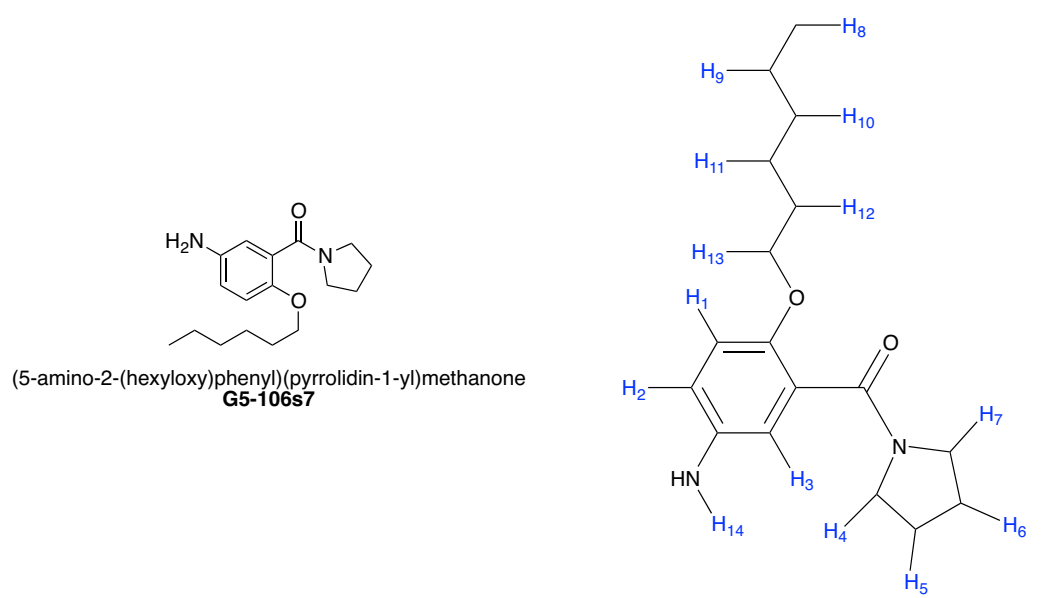

TABLE A.15: ${ }^{1} \mathrm{H}-\mathrm{NMR}$ assignments for G5-106s7, $400 \mathrm{MHz}$ in DMSO (2.50 ppm)

\begin{tabular}{|c|c|c|c|c|c|}
\hline Proton & ppm $^{\mathrm{a}}$ & Number & Type & $\mathrm{J}_{\mathbf{a}}$ & $\mathbf{J}_{\mathbf{b}}$ \\
\hline $\mathrm{H}_{1}$ & 6.75 & 1 & $\mathrm{~d}$ & 8.70 & \\
\hline $\mathrm{H}_{2}$ & 6.54 & 1 & dd & 8.68 & 2.80 \\
\hline $\mathrm{H}_{3}$ & 6.38 & 1 & $\mathrm{~d}$ & 2.78 & \\
\hline $\mathrm{H}_{4}$ & 3.13 & 2 & $\mathrm{~m}$ & & \\
\hline $\mathrm{H}_{5} \& \mathrm{H}_{6}$ & 1.81 & 4 & $\mathrm{~m}$ & & \\
\hline $\mathrm{H}_{7}$ & 3.39 & 2 & $\mathrm{t}$ & 6.88 & \\
\hline $\mathrm{H}_{8}$ & 0.87 & 3 & $\mathrm{t}$ & 6.72 & \\
\hline $\mathrm{H}_{9}$ \& $_{10}$ & 2.89 & 4 & $\mathrm{~m}$ & & \\
\hline $\mathrm{H}_{11}$ & 1.36 & 2 & $\mathrm{~m}$ & & \\
\hline $\mathrm{H}_{12}$ & 1.60 & 2 & $\mathrm{~m}$ & & \\
\hline $\mathrm{H}_{13}$ & 3.82 & 2 & $\mathrm{t}$ & 6.23 & \\
\hline $\mathrm{H}_{14}$ & & 2 & $\mathrm{~s}$ & & \\
\hline
\end{tabular}

a. Taken at the center of the peak. 
(5-amino-2-(hexyloxy)phenyl)(pyrrolidin-1-yl)methone: G5-106s7

FIGURE A.55: ${ }^{1}$ H-NMR of G5-106s 7 .

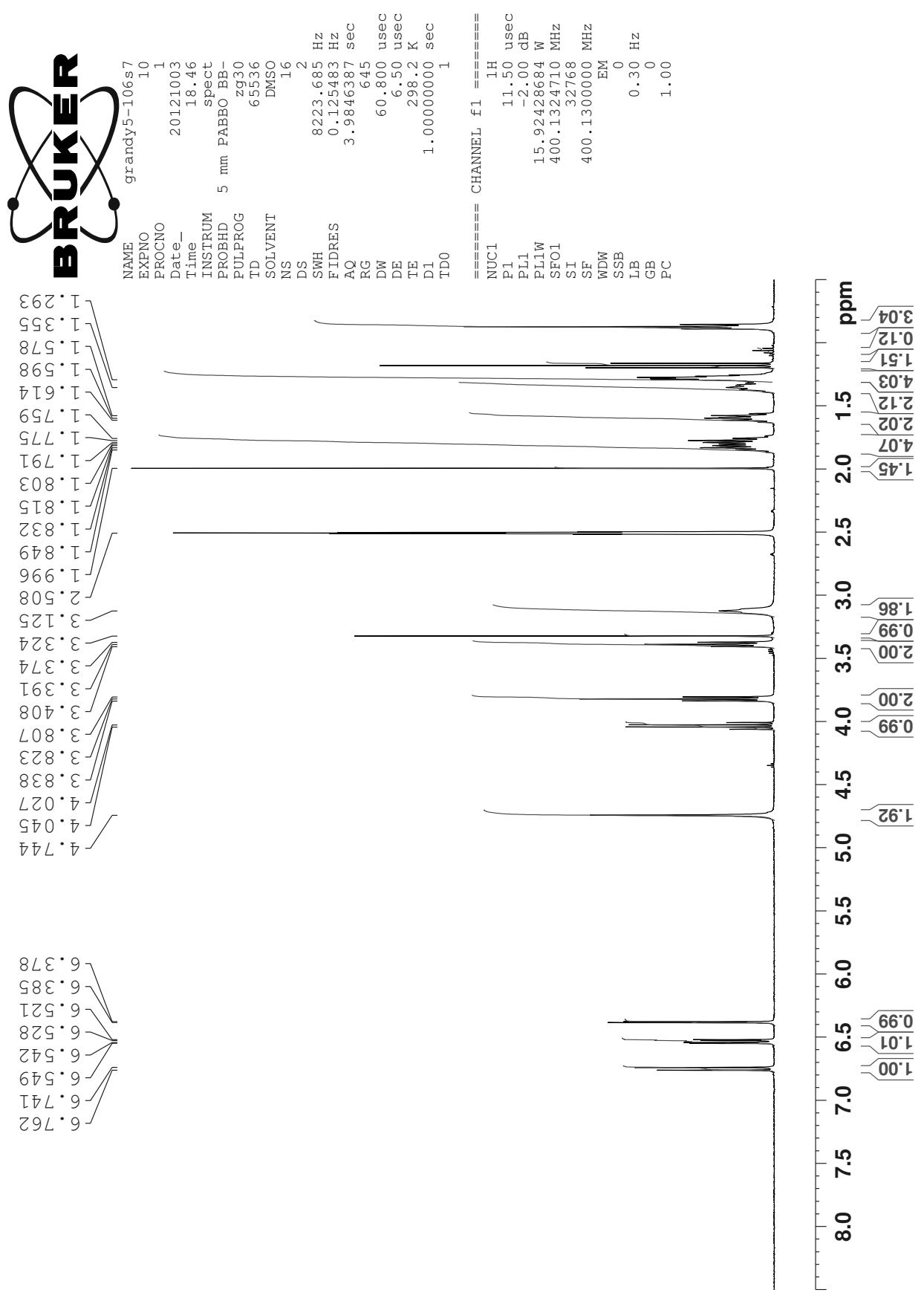


(5-amino-2-(hexyloxy)phenyl)(pyrrolidin-1-yl)methone: G5-106s7

FIGURE A.56: COSY-NMR of G5-106s7.

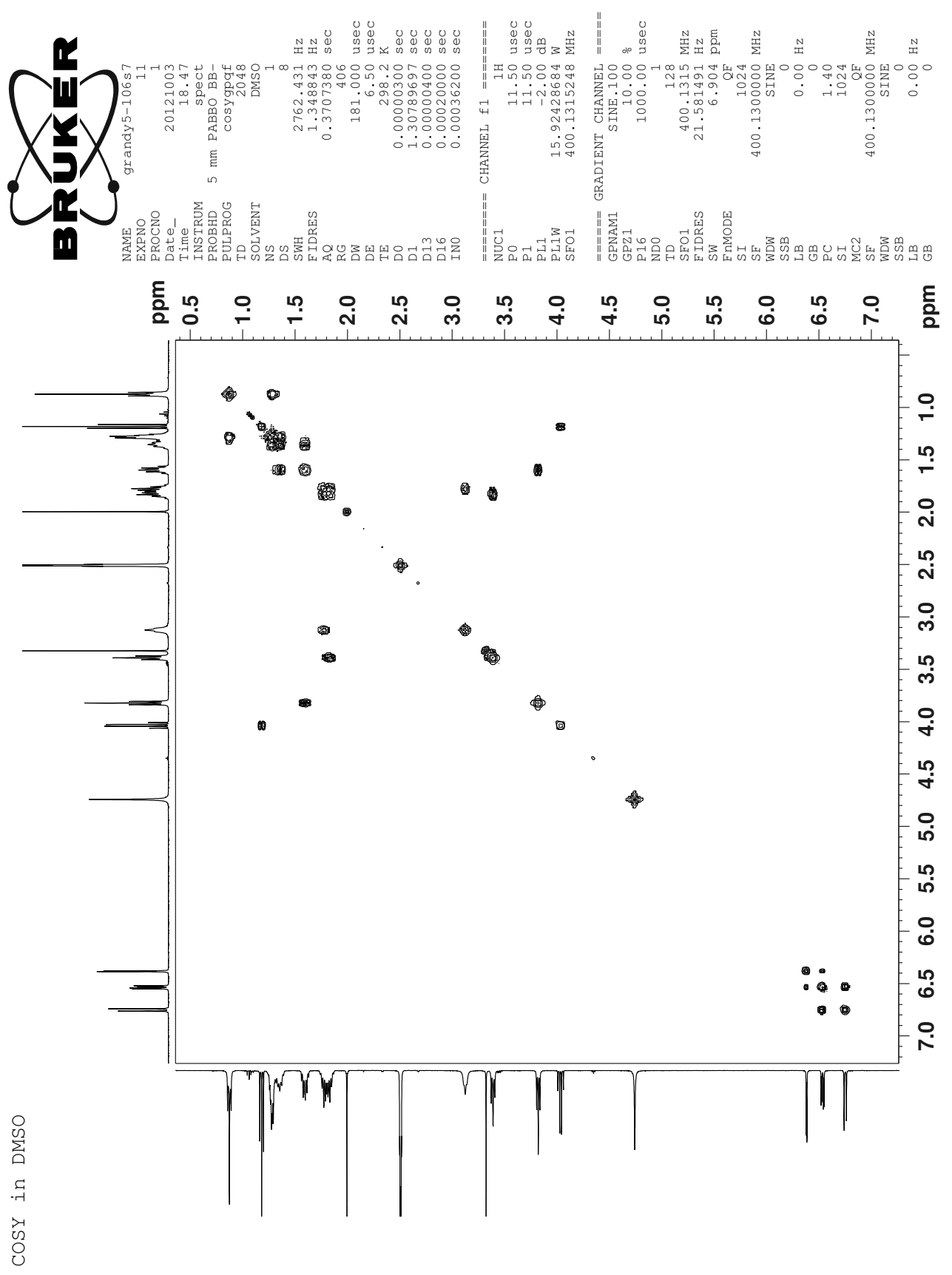


(5-((3-fluorobenzyl)amino)-2-(hexyloxy)phenyl)(pyrrolidin-1-yl)methone: G5-109s8

\section{A.9 (5-((3-fluorobenzyl)amino)-2-(hexyloxy)phenyl)(pyrrolidin-1-yl)methone:} G5-109s8

FIGURE A.57: Structure of G5-109s8.

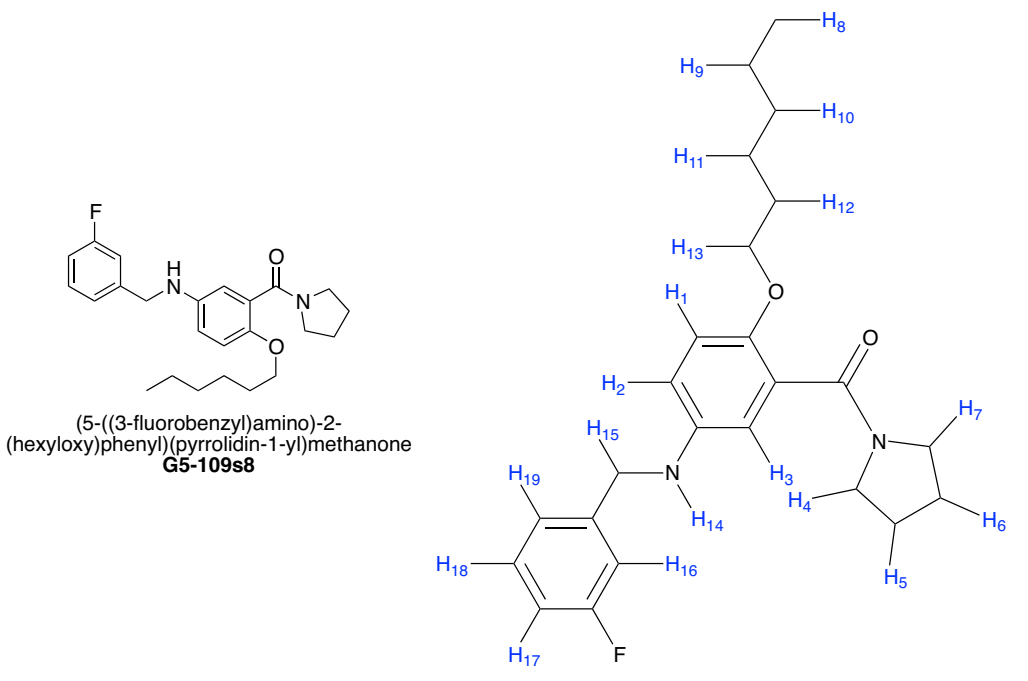

TABLE A.16: ${ }^{1} \mathrm{H}-\mathrm{NMR}$ assignments for G5-109s8, $400 \mathrm{MHz}$ in DMSO (2.50 ppm)

\begin{tabular}{|c|c|c|c|c|c|c|c|c|c|c|c|}
\hline Proton & $\mathbf{p p m}^{\mathbf{a}}$ & Number & Type & $\mathbf{J}_{\mathbf{a}}$ & $\mathbf{J}_{\mathbf{b}}$ & Proton & ppm $^{a}$ & Number & Type & $\mathbf{J}_{\mathbf{a}}$ & $\mathbf{J}_{\mathbf{b}}$ \\
\hline $\mathbf{H}_{1}$ & 6.80 & 1 & d & 8.84 & & $\mathrm{H}_{12}$ & 1.60 & 2 & m & & \\
\hline $\mathbf{H}_{2}$ & 6.53 & 1 & dd & 8.83 & 2.89 & $\mathrm{H}_{13}$ & 3.82 & 2 & $\mathbf{t}$ & 6.22 & \\
\hline $\mathbf{H}_{3}$ & 6.39 & 1 & d & 2.86 & & $\mathrm{H}_{14}$ & 6.02 & 1 & $\mathbf{t}$ & 6.23 & \\
\hline $\mathbf{H}_{4}$ & 3.06 & 2 & $\mathbf{m}$ & & & $\mathrm{H}_{15}$ & 4.24 & 2 & d & 6.18 & \\
\hline $\mathrm{H}_{5} \& \mathrm{H}_{6}$ & 1.78 & 4 & m & & & $\mathrm{H}_{16}$ & 7.15 & 1 & m & & \\
\hline $\mathbf{H}_{7}$ & 3.38 & 2 & $\mathbf{t}$ & 6.80 & & $\mathbf{H}_{17}$ & 7.04 & 1 & m & & \\
\hline $\mathrm{H}_{8}$ & 0.87 & 3 & $\mathbf{m}$ & 6.72 & & $\mathrm{H}_{18}$ & 7.36 & 1 & $\mathbf{m}$ & & \\
\hline$H_{9} \& H_{10}$ & 1.27 & 4 & m & & & $\mathrm{H}_{19}$ & 7.20 & 1 & m & & \\
\hline $\mathrm{H}_{11}$ & 1.35 & 2 & $\mathbf{m}$ & & & - & - & - & - & - & - \\
\hline
\end{tabular}

a. Taken at the center of the peak. 
(5-((3-fluorobenzyl)amino)-2-(hexyloxy)phenyl)(pyrrolidin-1-yl)methone: G5-109s8

FIGURE A.58: ${ }^{1}$ H-NMR of G5-109s9.

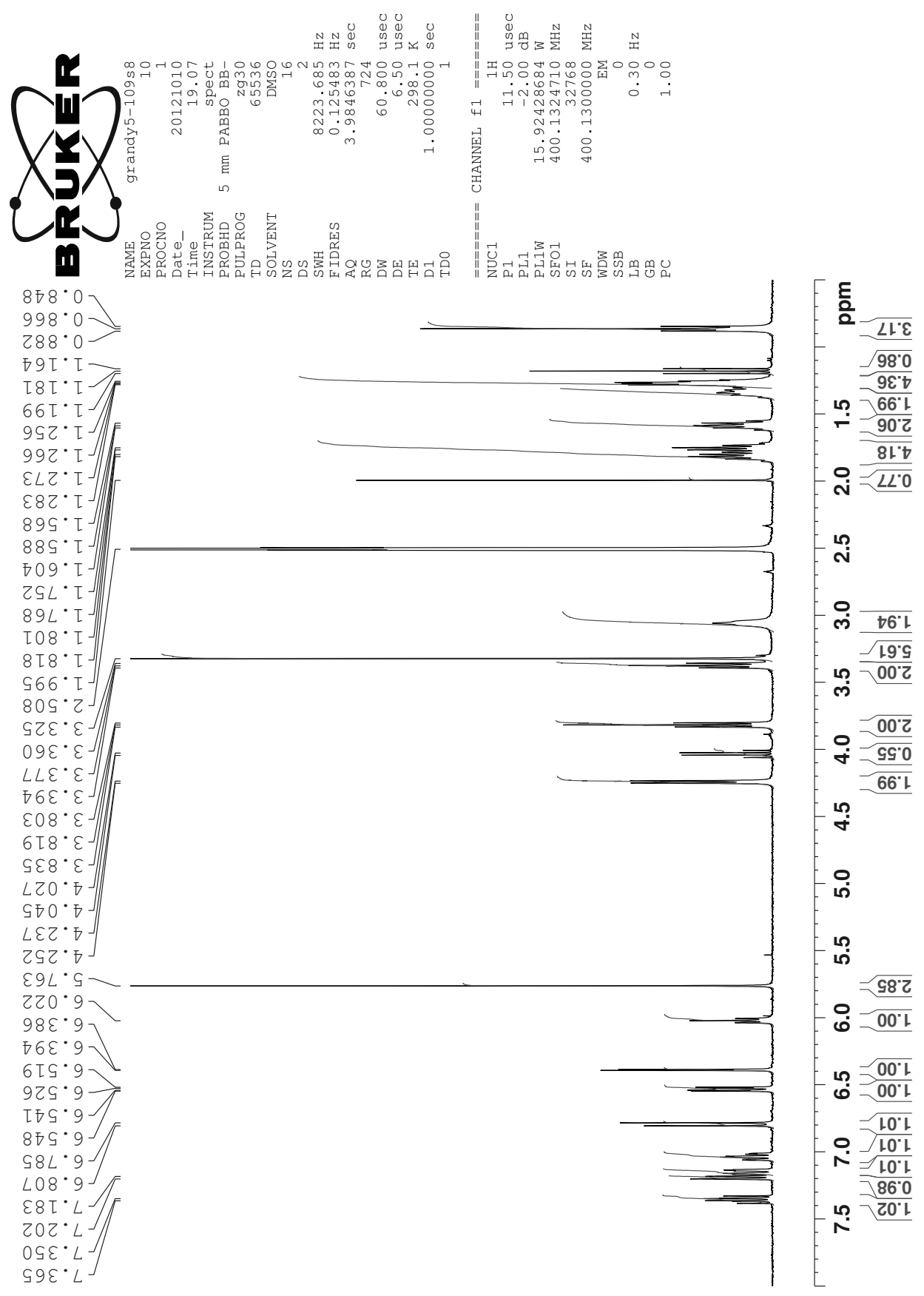


(5-((3-fluorobenzyl)amino)-2-(hexyloxy)phenyl)(pyrrolidin-1-yl)methone: G5-109s8

FIGURE A.59: COSY-NMR of G5-109s9.

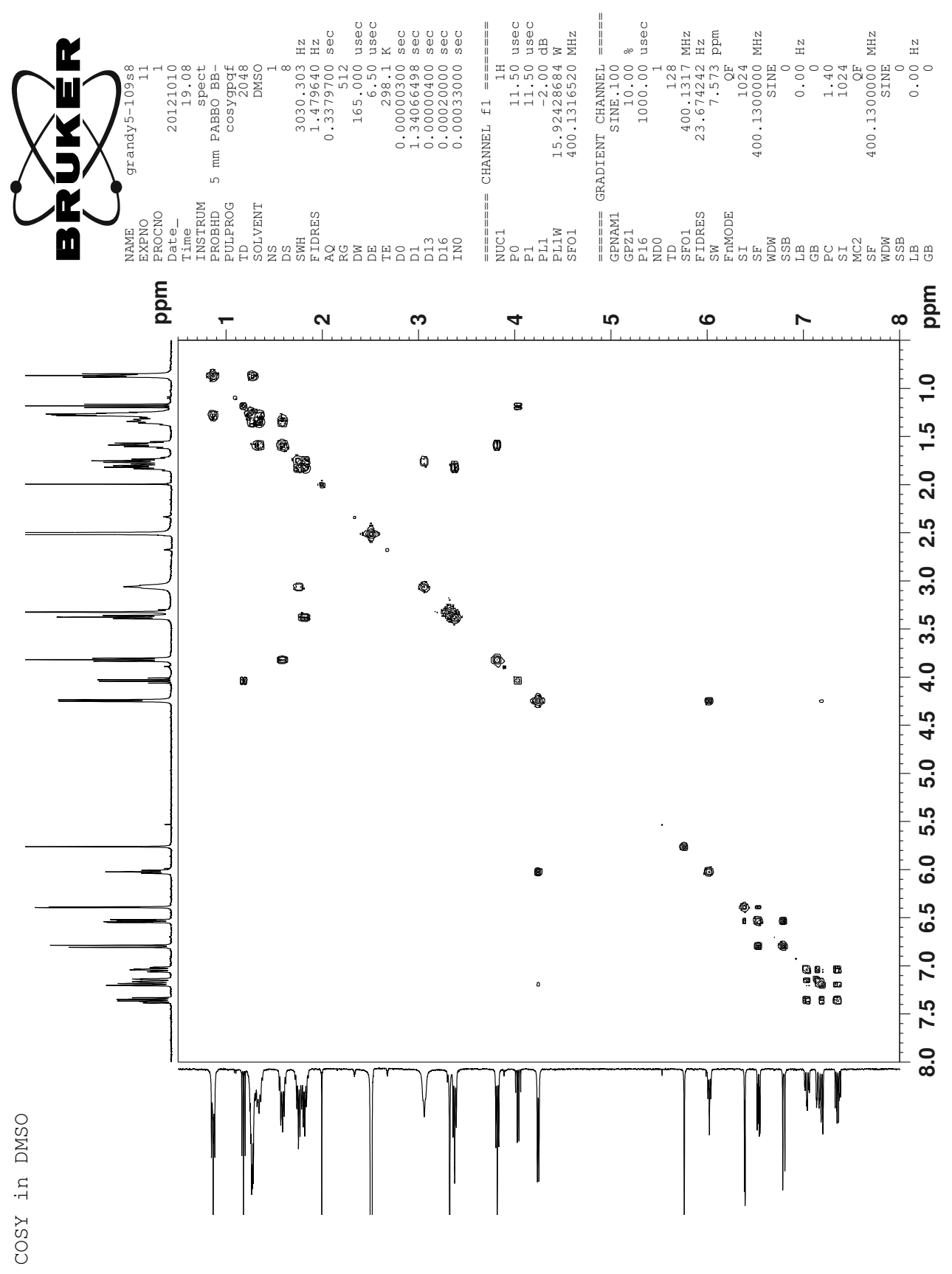


(5-((3-fluorobenzyl)amino)-2-(hexyloxy)phenyl)(pyrrolidin-1-yl)methone: G5-109s8

FIGURE A.60: ${ }^{19} \mathrm{~F}-\mathrm{NMR}$ of G5-109s9, $400 \mathrm{MHz}$ with $\mathrm{C}_{6} \mathrm{~F}_{6}$ (-164.0 ppm).

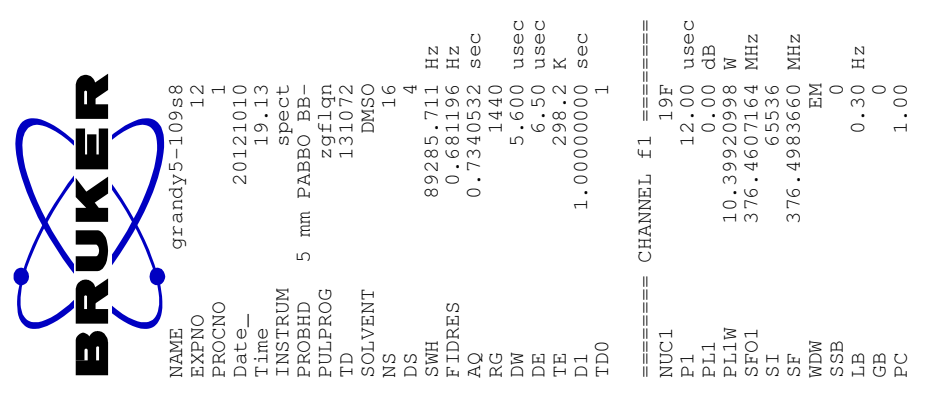

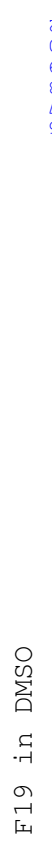
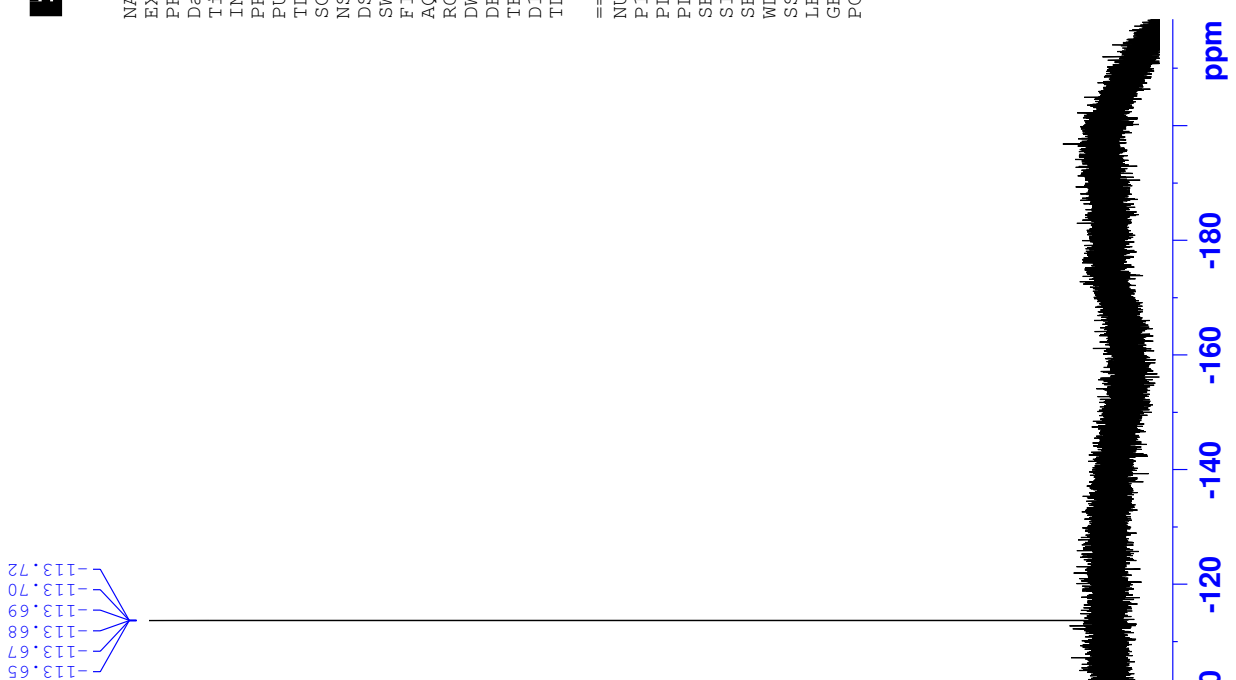


\section{B.1 5-bromo-2-(hexyloxy)benzaldehyde: G4-012s16}

FIGURE B.1: Ionic forms of G4-012s16.

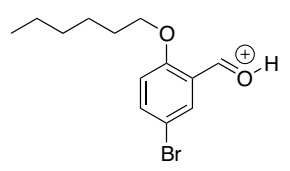

1

Chemical Formula: $\mathrm{C}_{13} \mathrm{H}_{18} \mathrm{BrO}_{2}{ }^{+}$ Exact Mass: 285.05

m/z: 285.05 (100.0\%), 287.05 (97.7\%), 286.05 (14.1\%), 288.05 (14.0\%

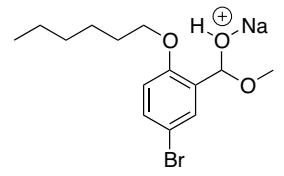

3

Chemical Formula: $\mathrm{C}_{14} \mathrm{H}_{21} \mathrm{BrNaO}_{3}{ }^{+}$ Exact Mass: 339.06

m/z: 339.06 (100.0\%), 341.06 (99.0\%), 340.06 (15.5\%), 342.06 (15.2\%)

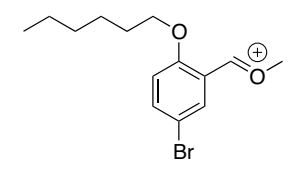

Chemical Formula: $\mathrm{C}_{14} \mathrm{H}_{20} \mathrm{BrO}_{2}{ }^{+}$ Exact Mass: 299.06 m/z: 299.06 (100.0\%), 301.06 (97.3\%), 300.07 (15.4\%), 302.07 (15.1\%)

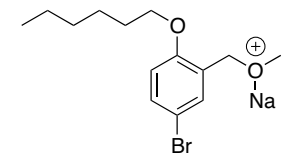

Chemical Formula: $\mathrm{C}_{14} \mathrm{H}_{21} \mathrm{BrNaO}_{2}{ }^{+}$

Exact Mass: 323.06

m/z: 323.06 (100.0\%), 325.06 (97.3\%), 324.07 (15.5\%), 326.06 (14.8\%) 


\section{5-bromo-2-(hexyloxy)benzaldehyde: G4-012s16}

FIGURE B.2: Mass spectrum of G4-012s16. The red numbers refer to the ionic forms in FIGURE B.1.

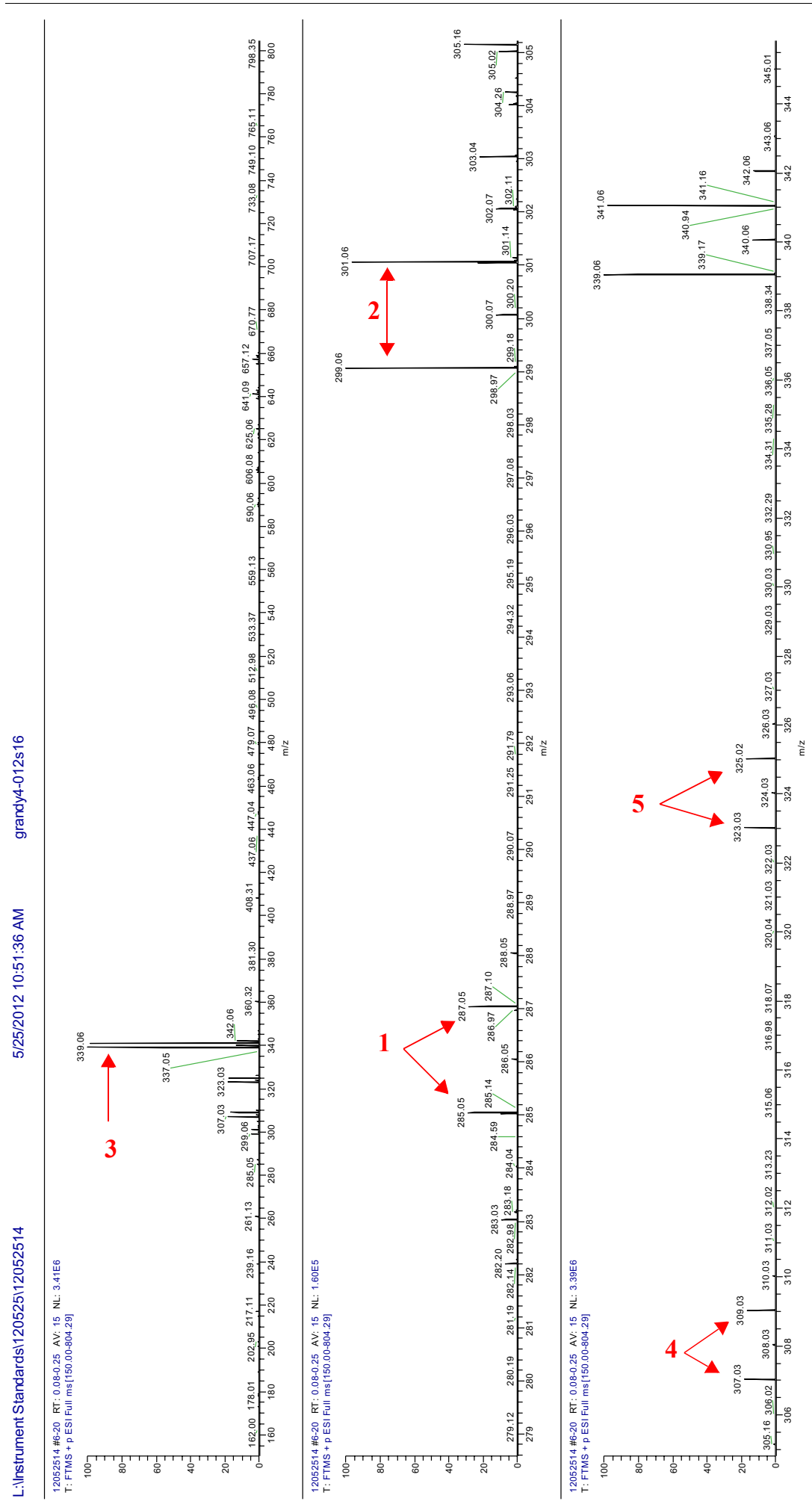


5-(4-fluorophenoxy)-2-(hexyloxy)benzaldehyde: G4-068s20 \& G4-070s6

\section{B.2 5-(4-fluorophenoxy)-2-(hexyloxy)benzaldehyde: G4-068s 20 \& G4-070s6}

FIGURE B.3: Ionic forms of G4-070s6.

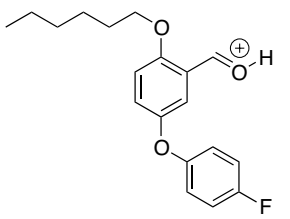

1

Chemical Formula: $\mathrm{C}_{19} \mathrm{H}_{22} \mathrm{FO}_{3}{ }^{+}$ Exact Mass: 317.15 $\mathrm{m} / \mathrm{z}: 317.16(100.0 \%), 318.16(20.9 \%), 319.16(2.7 \%)$

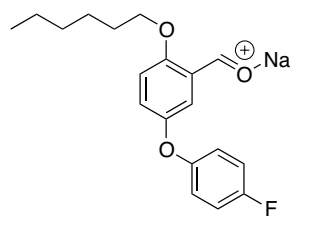

Chemical Formula: $\mathrm{C}_{19} \mathrm{H}_{21} \mathrm{FNaO}_{3}+$ Exact Mass: 339.14

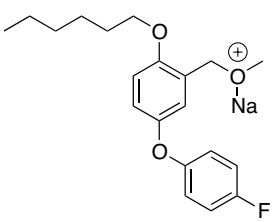

3

Chemical Formula: $\mathrm{C}_{20} \mathrm{H}_{25} \mathrm{FNaO}_{3}{ }^{+}$ Exact Mass: 355.17

$\mathrm{m} / \mathrm{z}: 355.17$ (100.0\%), $356.17(22.0 \%), 357.18(2.3 \%)$ 
5-(4-fluorophenoxy)-2-(hexyloxy)benzaldehyde: G4-068s20 \& G4-070s6

FIGURE B.4: Mass spectrum of G4-068s20. The red numbers refer to the ionic forms in FIGURE B.3.

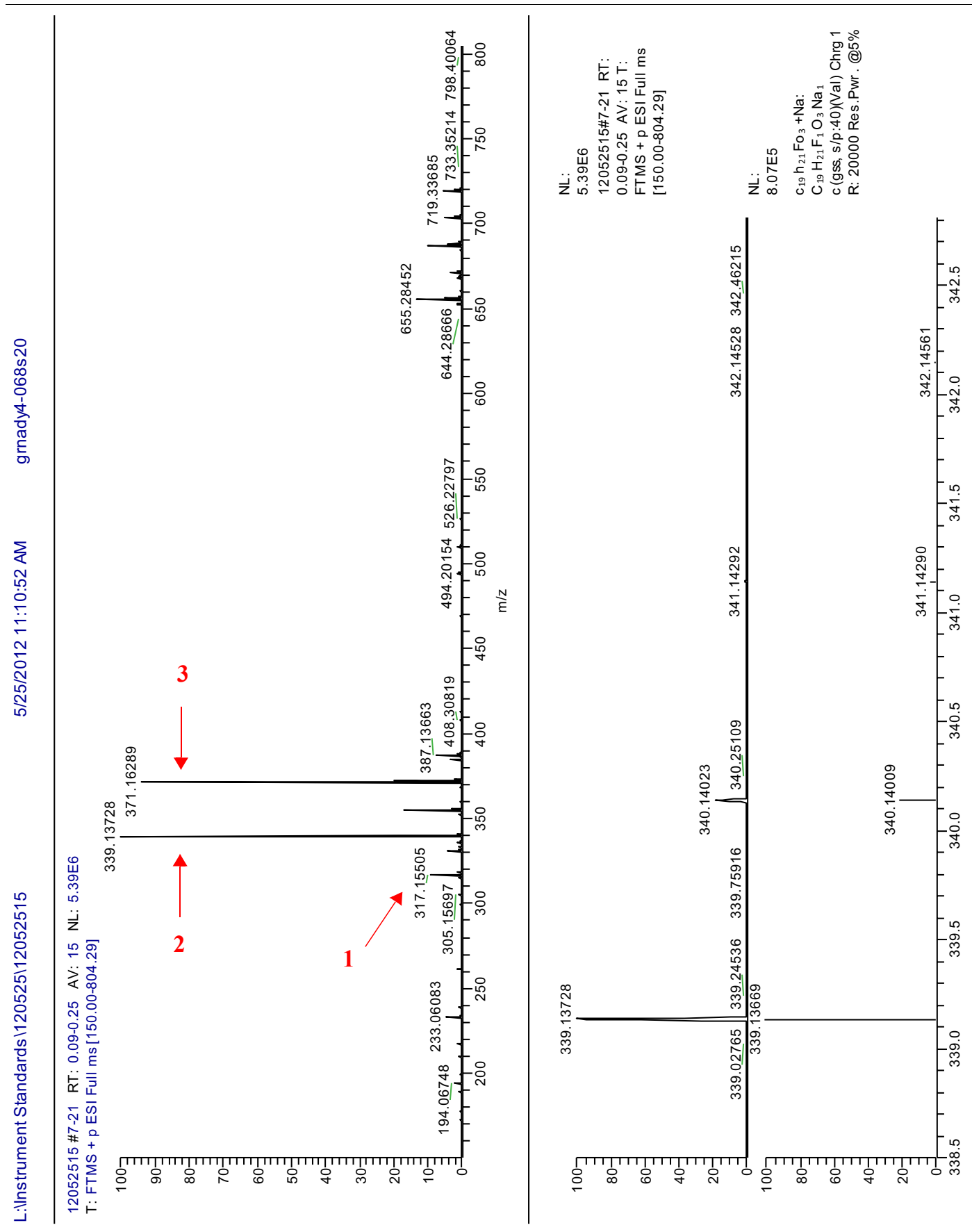


(5-(4-fluorophenoxy)-2-(hexyloxy)phenyl)(phenyl)methanol: G4-074s14

\section{B.3 (5-(4-fluorophenoxy)-2-(hexyloxy)phenyl)(phenyl)methanol: G4-074s14}

FIGURE B.5: Ionic forms of G4-074s14.

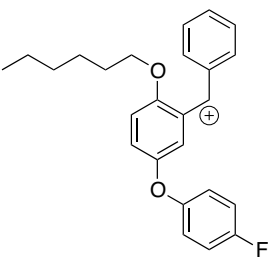

Chemical Formula: $\mathrm{C}_{25} \mathrm{H}_{26} \mathrm{FO}_{2}{ }^{+}$ Exact Mass: 377.19 m/z: $377.19(100.0 \%), 378.20(27.4 \%), 379.20(4.0)$

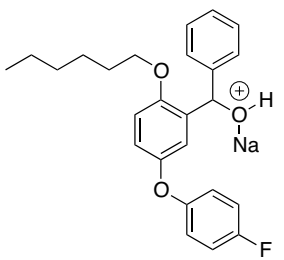

Chemical Formula: $\mathrm{C}_{25} \mathrm{H}_{27} \mathrm{FNaO}_{3}{ }^{+}$ Exact Mass: 417.18

$\mathrm{m} / \mathrm{z}: 417.18(100.0 \%), 418.19(27.5 \%), 419.19(4.2 \%)$ 
(5-(4-fluorophenoxy)-2-(hexyloxy)phenyl)(phenyl)methanol: G4-074s14

FIGURE B.6: Mass spectrum of G4-074s14. The red numbers refer to the ionic forms in FIGURE B.5.

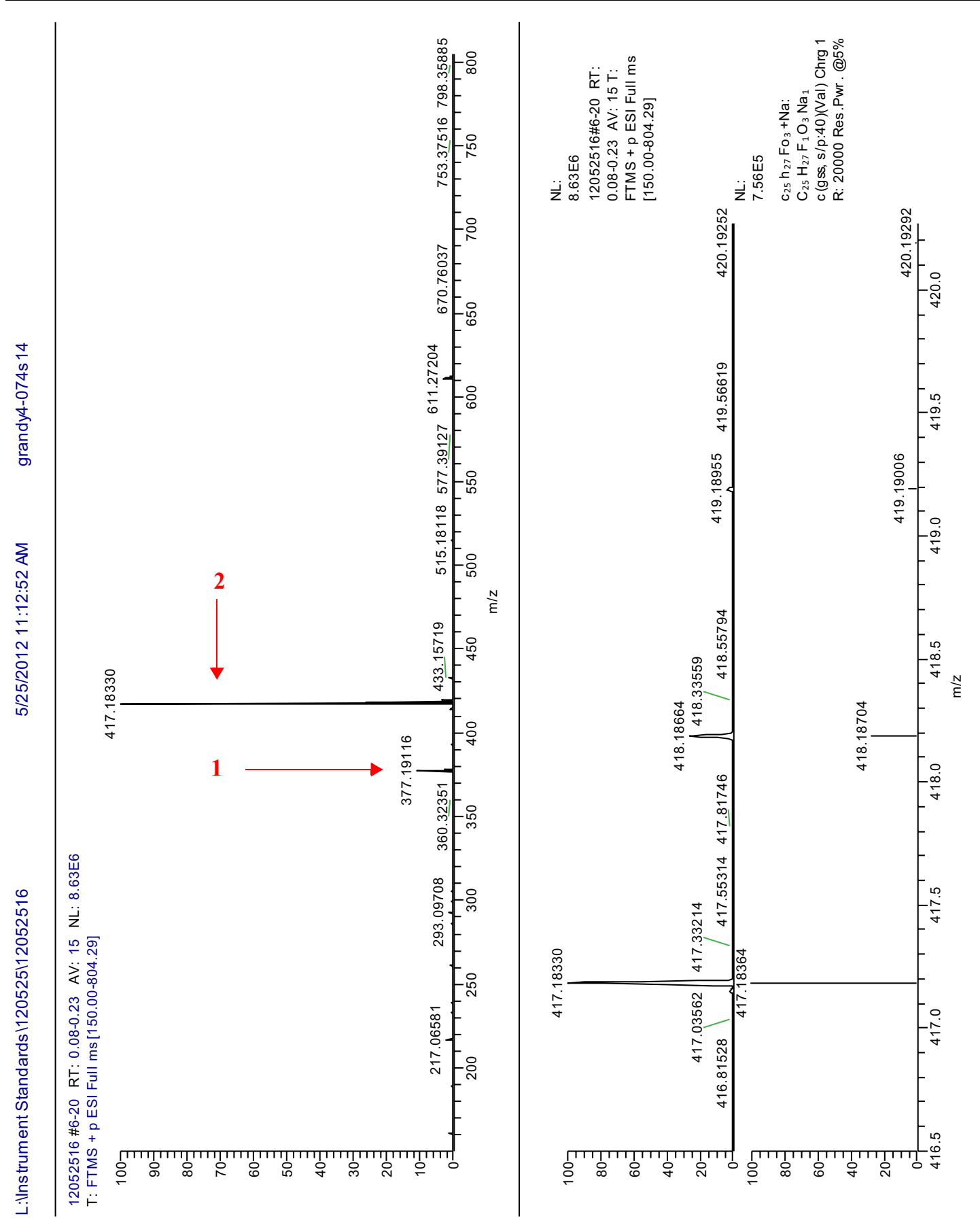


2-(5-(4-fluorophenoxy)-2-(hexyloxy)phenyl)-2-phenylacetonitrile: G4-079s9

B.4 2-(5-(4-fluorophenoxy)-2-(hexyloxy)phenyl)-2-phenylacetonitrile: G4-079s9

FIGURE B.7: Ionic form of G4-079s9.

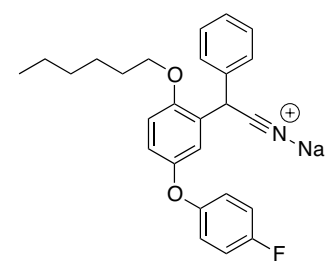

Chemical Formula: $\mathrm{C}_{26} \mathrm{H}_{26} \mathrm{FNNaO}_{2}{ }^{+}$ Exact Mass: 426.18

$\mathrm{m} / \mathrm{z}: 426.18(100.0 \%), 427.19(28.5 \%), 428.19(4.3 \%)$ 
2-(5-(4-fluorophenoxy)-2-(hexyloxy)phenyl)-2-phenylacetonitrile: G4-079s9

FIGURE B.8: Mass spectrum of G4-079s9. The red number refers to the ionic form in FIGURE B.7.

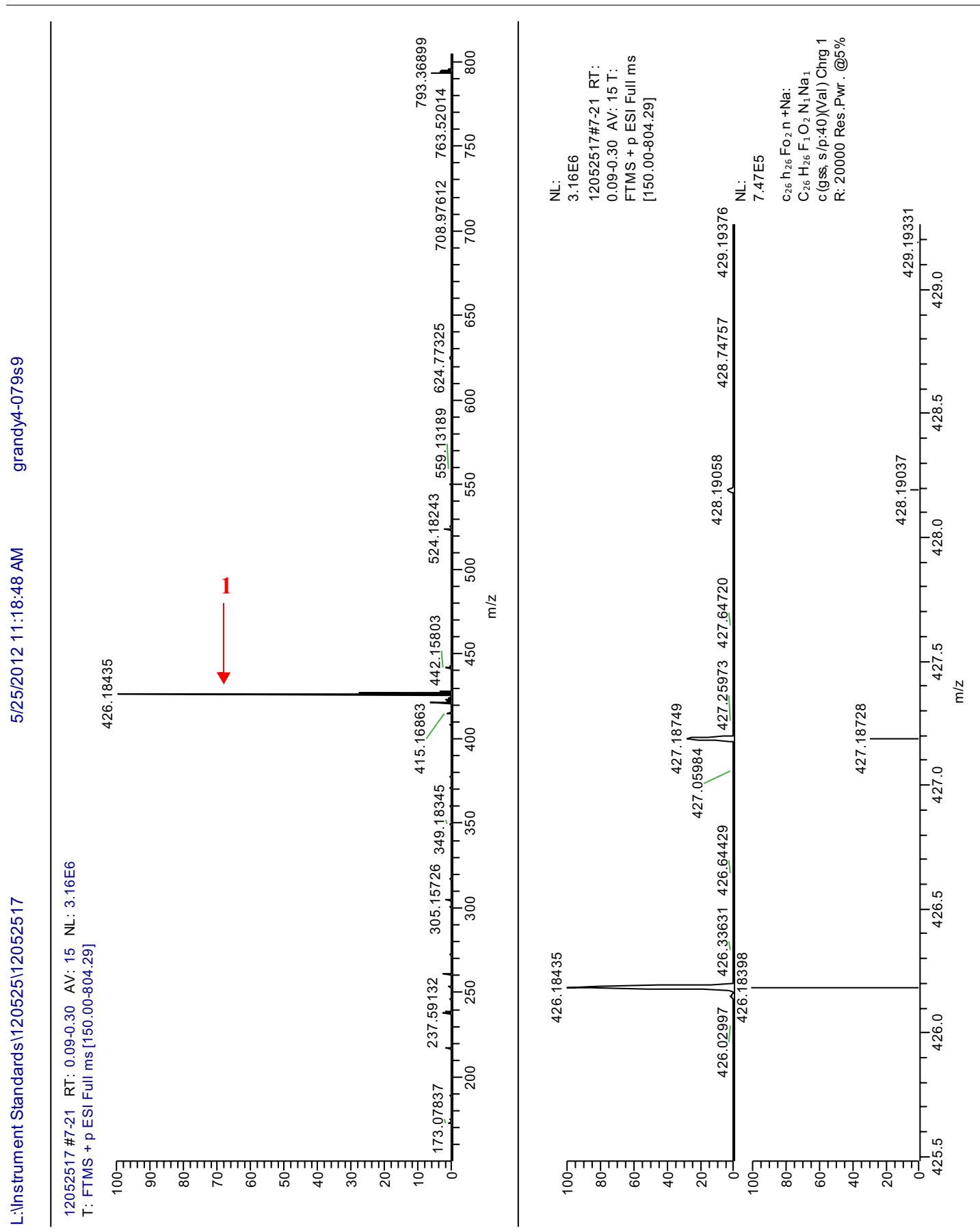


ET-92: 2-(5-(4-fluorophenoxy)-2-(hexyloxy)phenyl)-2-phenylethanamine hydrochlo-

\section{B.5 ET-92: 2-(5-(4-fluorophenoxy)-2-(hexyloxy)phenyl)-2-phenylethanamine} hydrochloride: G4-091s14

FIGURE B.9: Ionic form of ET-92 (G4-090s14).

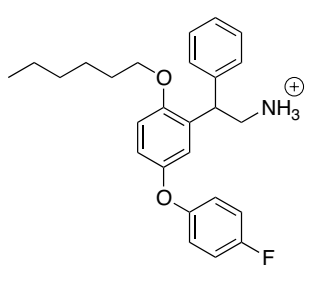

Chemical Formula: $\mathrm{C}_{26} \mathrm{H}_{31} \mathrm{FNO}_{2}{ }^{+}$

Exact Mass: 408.23

$\mathrm{m} / \mathrm{z}: 408.23(100.0 \%), 409.24$ (28.6\%), 410.24 (4.3\%) 
ET-92: 2-(5-(4-fluorophenoxy)-2-(hexyloxy)phenyl)-2-phenylethanamine hydrochlo-

FIGURE B.10: Mass spectrum of ET-92. The red number refers to the ionic form in FIGURE B.9.

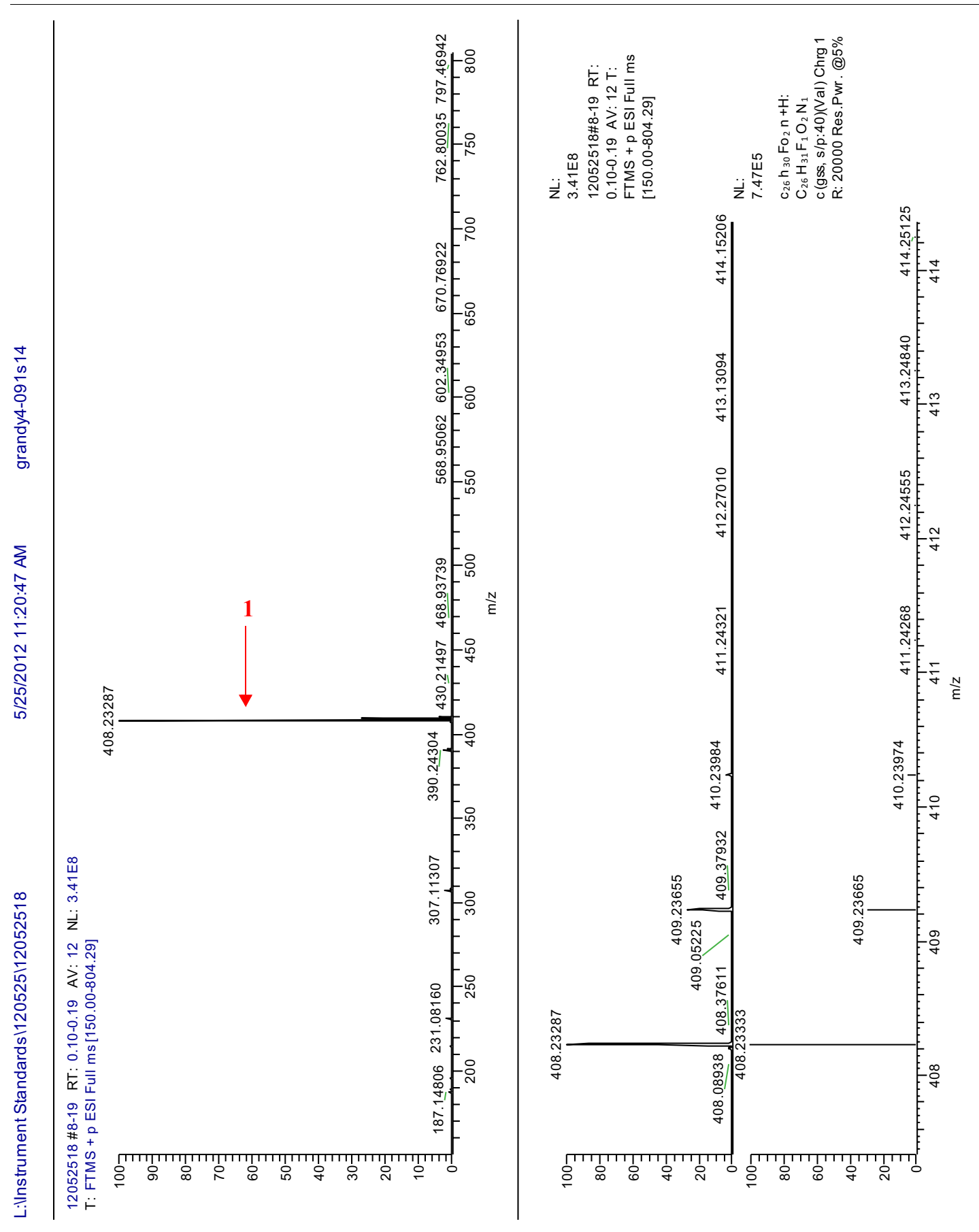

